\title{
Phobia: contingencies, cognitions, and reflexes
}

Citation for published version (APA):

de Jong, P. J. (1994). Phobia: contingencies, cognitions, and reflexes. [Doctoral Thesis, Maastricht University]. Datawyse / Universitaire Pers Maastricht. https://doi.org/10.26481/dis.19940916pj

Document status and date:

Published: 01/01/1994

DOI:

10.26481/dis.19940916pj

Document Version:

Publisher's PDF, also known as Version of record

\section{Please check the document version of this publication:}

- A submitted manuscript is the version of the article upon submission and before peer-review. There can be important differences between the submitted version and the official published version of record.

People interested in the research are advised to contact the author for the final version of the publication, or visit the DOI to the publisher's website.

- The final author version and the galley proof are versions of the publication after peer review.

- The final published version features the final layout of the paper including the volume, issue and page numbers.

Link to publication

\footnotetext{
General rights rights.

- You may freely distribute the URL identifying the publication in the public portal. please follow below link for the End User Agreement:

www.umlib.nl/taverne-license

Take down policy

If you believe that this document breaches copyright please contact us at:

repository@maastrichtuniversity.nl

providing details and we will investigate your claim.
}

Copyright and moral rights for the publications made accessible in the public portal are retained by the authors and/or other copyright owners and it is a condition of accessing publications that users recognise and abide by the legal requirements associated with these

- Users may download and print one copy of any publication from the public portal for the purpose of private study or research.

- You may not further distribute the material or use it for any profit-making activity or commercial gain

If the publication is distributed under the terms of Article $25 \mathrm{fa}$ of the Dutch Copyright Act, indicated by the "Taverne" license above, 
PHOBIA:

CONTINGENCIES, COGNITIONS, AND REFLEXES 
Nog een blaadje

Plotseling stond het boompje

Nakend in de zon

(Japanse Haiku; bron onbekend) 


\section{PHOBIA:}

\section{CONTINGENCIES, COGNITIONS, AND REFLEXES}

\section{PROEFSCHRIFT}

ter verkrijging van de graad van doctor aan de Rijksuniversiteit Limburg te Maastricht, op gezag van de Rector Magnificus, Prof. Dr. H. Philipsen, volgens het besluit van het College van Dekanen, in het openbaar te verdedigen op

vrijdag 16 september 1994 om 14.00 uur

$$
\text { door }
$$

Pieter Job de Jong

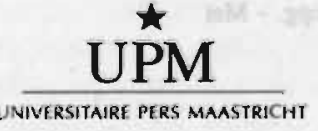




\section{Promotor}

Prof. Dr. M.A. van den Hout

\section{Co-promotor}

Dr. H. Merckelbach

\section{Beoordelingscommissie}

Prof. Dr. G.J. Kok (voorzitter)

Prof. Dr. H.F.M. Crombag

Prof. Dr. P. Eelen (Katholieke Universiteit Leuven)

Prof. Dr. R. McNally (Harvard University)

Prof. Dr. H.G. Schmidt

\section{Druk}

Datawyse Maastricht

CIP-DATA KONINKLIJKE BIBLIOTHEEK, DEN HAAG

Jong, Pieter Job de

Phobia: contingencies, cognitions, and reflexes / Pieter

Job de Jong. - Maastricht : Universitaire Pers

Maastricht. - III.

Proefschrift Maastricht. - Met lit. opg. - Met

samenvatting in het Nederlands.

ISBN 90-5278-151-6

Trefw.: fobieen. 
2 The Phenomena I: Covariation Bias and Phobia

a) A First Replication

b) A Second Replication

3 Pathways to Covariation Bias

a) Suggestive Coincidences 41

b) A Priori Expectancies I 53

c) A Priori Expectancies II 61

d) Hemisphericity and Imagination $\quad 79$

4 Covariation Bias: Cause or Consequence?

a) Covariation Bias and the Return of Fear

5 The Phenomena II: UCS-Revaluation and Phobia
a) UCS-Inflation
b) Modulating Factors

6 The Phenomena III: Startle Modulation and Phobia

a) Startle and Phobia I

b) Starlle and Phobia II

7 Contingencies, Cognitions, and Reflexes

a) A Recapitulation

References

Samenvatting

Dankwoord 


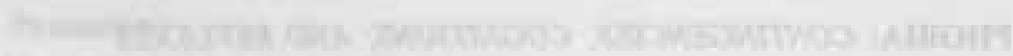

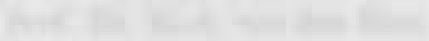

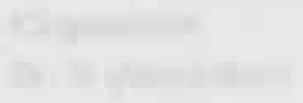

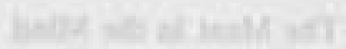

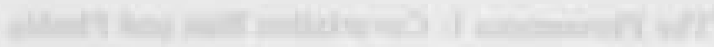

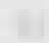

in

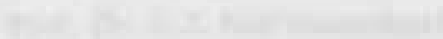

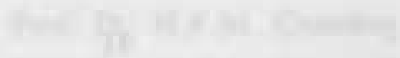

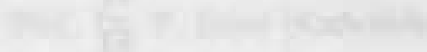

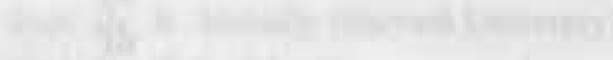

(n)

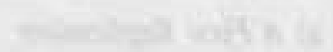

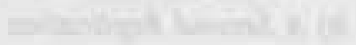

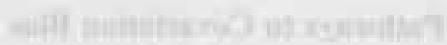

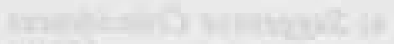

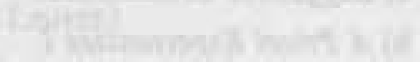

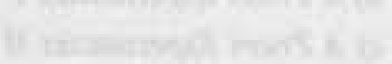
and

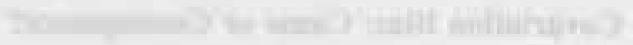

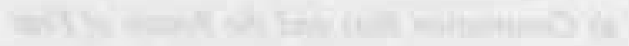

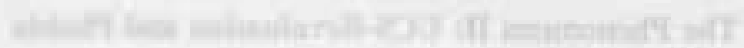

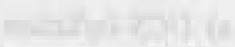

$+1$

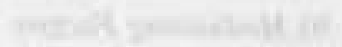

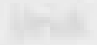

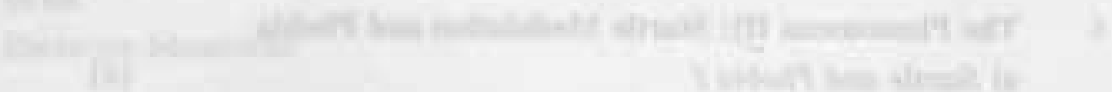

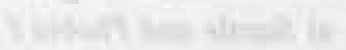




\section{The Meat in the Mind}

\section{Perception of Patterns}

Early studies on human perception clearly showed that perception is not just a passive phenomenon; perception can not be properly understood as a bottom up process in which external stimuli are merely reflected to the brain. That perception is more than a direct registration of sensations was already acknowledged by William James (1890) more than a century ago. In his Principles of Psychology, he contained that although a part of what we perceive comes through our senses from the external objects, another part (and it may be the larger part) always comes out of our head.

Several studies have provided experimental support for the hypothesis that the relationship between sensations and perception is mediated by cognitive processes. Figure 1.1 is one illustration of top-down processes affecting perception.

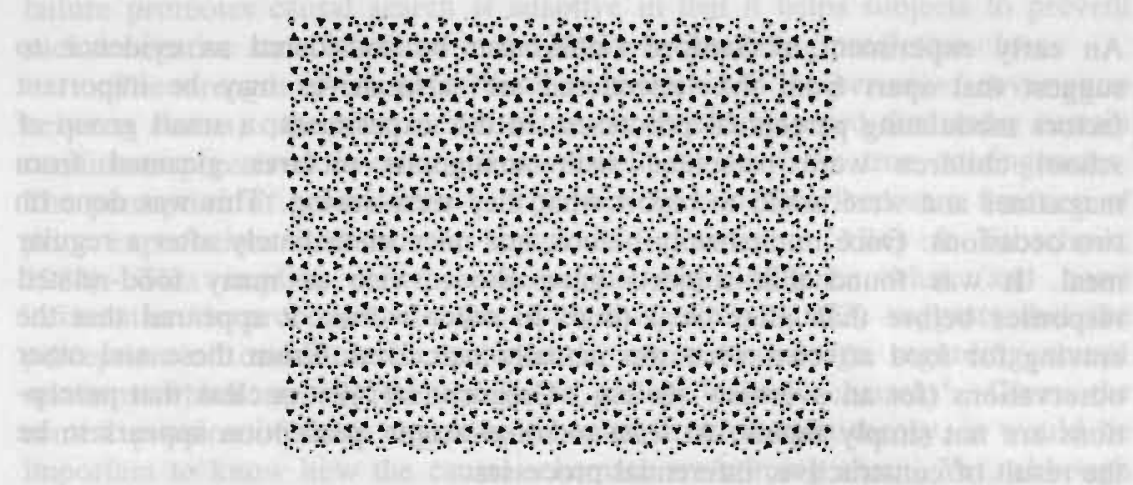

Figure 1.1: Derived from S. Coren \& L.M. Ward (1989).

In this ambiguous figure the dots are part of several patterns, while none of the patterns is clearly dominating. Looking at this figure irresistably results in perceiving alternating configurations ranging from circles to crosses of different sizes and complexity. Figure 1.2 is another demonstration that perception is open to control by central factors. At first glance, Figure 1.2 seems to depict a random pattern of black shapes on a white ground. However, after receiving the appropriate hints, most readers will recognize the 
Christ-like face that is hidden in this figure. Once discovered, the face will always emerge when looking to this figure. These observations seem to indicate that perception is guided by attentional factors.

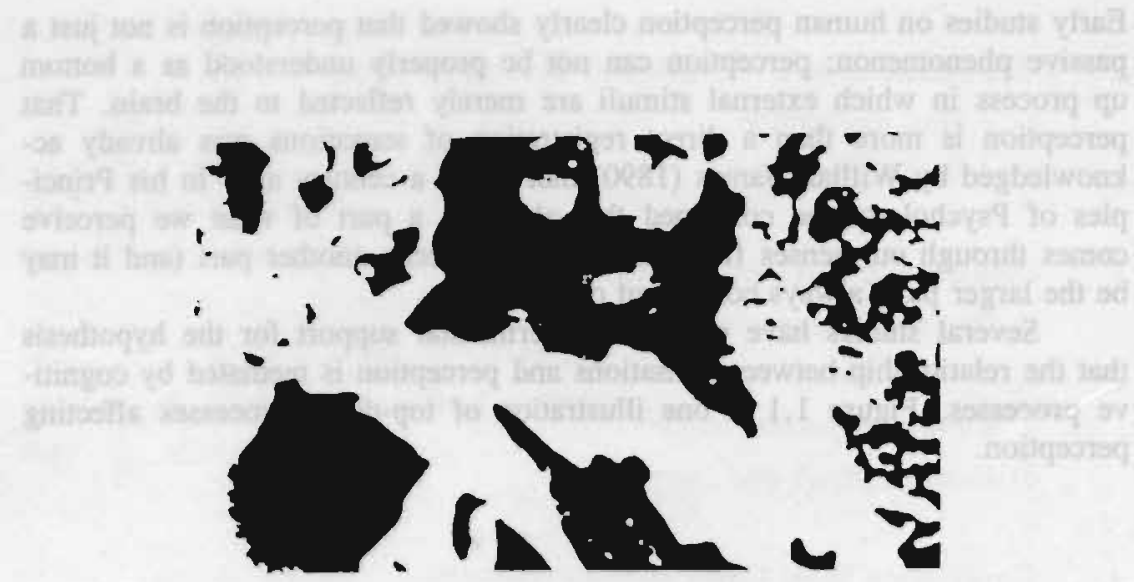

Figure 1.2: Copied from I.E. Gordon (1989).

An early experiment of Sanford (1936) can be considered as evidence to suggest that apart from the "attentional set" also drives may be important factors modulating perceptual processes. In this experiment, a small group of school children were presented with ambiguous pictures gleamed from magazines and were asked to report what they were seeing. This was done on two occasions: Once immediately before, and once immediately after a regular meal. It was found that subjects gave about twice as many food-related responses before than after meal time. In other words, it appeared that the craving for food affected what was actually perceived. From these and other observations (for an overview see e.g., Gregory, 1974) it is clear that perceptions are not simply inputs. At least to some extent, perception appears to be the result of constructive, inferential processes.

The readiness to impose patterns on the external world is not restricted to the perception of discrete stimuli or isolated events. Subjects also tend to perceive relationships between events. This can be nicely illustrated by the "experiment" of Karmel at the University of Pennsylvania (cited in Seligman \& Yellen, 1987). During a colloquium he crumbled a string of christmas lights that were blinking in a random order. However, from the moment that Karmel put on some music of the Beatles, the students were overtaken by the illusion that the lights were now pulsating in time with the rythm of the music. Seemingly, the perception of the lights was structured by the beat of the Beatles, resulting in an illusory correlation between the blinking and the beat. 


\section{Covariation Detection}

The tendency to perceive relationships between co-occurring events is readily understandable from a functionalist perspective (e.g., Weiner, 1980). That is, knowledge regarding the covariation of events may have substantial survival value as it enables subjects to predict future events and to control the present. For effective coping it is important to locate predictors and/or causes of pertinent outcomes as marital loss, food deprivation, and bodily harm. Thus, covariation assessment can be considered adaptive to the extent that it serves to optimalize the likelihood of obtaining desired outcomes and avoiding aversive ones. From this perspective, the perception of illusory correlations may be considered as the cost of otherwise highly functional cognitive adaptations (cf., Arkes, 1991).

In the 1980s, several studies were carried out on spontaneous attributional activity (for a review see Weiner, 1985). The available evidence strongly suggests that the search for causal relationships is promoted by the occurrence of unexpected events (e.g., loss by favoured team) and by the nonattainment of a goal (e.g., rejection of a manuscript) (Weiner, 1985). That failure and the unexpected elicit attributional activity fits with a functionalist framework. Surprise-induced causal search can be conceptualised as exploratory behavior. Exploration can be considered adaptive as it enhances the understanding of the environment, thereby sustaining successful avoidance of aversive events. That failure promotes causal search is adaptive in that it helps subjects to prevent such a negative state of affairs in the future by providing predictive clues.

The strength of causal attributions is related to the perceived covariation between events, outcomes, or situations. It should be noted that under certain conditions perceived covariations may well deviate from true contingencies. The perception of covariations can perhaps be best described as a function of prior expectations and current situational information (Alloy \& Tabachnik, 1984). This implies that preconceptions may influence what (and how) information is processed. In line with this, Kelly (1973) suggests that the perception of causation is mediated by prior knowledge regarding causal relations which are represented in so called "causal schemata". Given the central position of these cognitive schemes in attribution theory, it would be important to know how the causal schemata are coming about. Yet, although attribution theorists (e.g., Kelly \& Michela, 1980) provide elaborate analyses of the causal schemata and the related attributional principles, they are relatively silent as to the mechanisms behind these schemata. As most attribution situations can be readily translated into conditioning terminology (event to be explained =UCS; possible cause $=C S$ ), Algom and Bizman (1983) propose that the empirical laws of recent associative learning theories would be good candidates for "...providing the missing motor for driving the attributional mechanism." (p. 772). In line with this suggestion, Alloy and Tabachnik (1984) convincingly demonstrate that human assessment of covariations may reflect learning processes similar to those observed in animal conditioning. Eelen (1982) also illustrate that there is a strong similarity between factors 
influencing attribution and factors affecting conditioned responding (see also Dickinson \& Shanks, 1985; Allan, 1993).

\section{Classical Conditioning and Contingency Judgments}

The "cognitive shifts" that underwent psychology in the 1960s did not leave learning theory unaffected. Gradually, the focus of learning theorists moved from strictly observable phenomena (the radical behavioural approach: "what is on your behavior") to the level of unobservable aspects of the conditioning mechanisms (Furedy \& Riley, 1987; Rapee, 1991). In line with this, stimulus/response relationships were the main topic of interest in the "precognitive" era, whereas contemporary theories of conditioning focus on the nature of the associations and, therefore, predominantly rely on stimulus/stimulus rather than stimulus/response relationships. In fact, currently the conditioned responses are predominantly used as a tool to infer what is learned rather than as phenomena that are of interest in their own right (but see Furedy, 1992).

This shift of focus was accompanied by several observations that were difficult to reconcile with the traditional S-R account of learning (e.g., Hull, 1943). The traditional learning theories considered conditioning primarily as a reflexive process: The conditioned stimulus (CS) was viewed to be a substitute for the unconditioned stimulus (UCS) in evoking the conditioned response (CR). Temporal contiguity and the inter-stimulus-interval were considered to be the crucial determinants of this process. One of the observations that seriously questioned whether the S-R account of conditioning adequately characterize the content of learning and the circumstances that produce learning is Kamin's "blocking" phenomenon (1969). In his animal experiments, Kamin first paired a CS1 with a UCS. In the subsequent phase a compound stimulus comprising the CS1 and a novel CS2 was paired with the same UCS. In the final test phase the rats were presented with CS2 only presentations. In contrast to control animals that were given no prior learning experiences with the CS1, the experimental animals showed only very weak responses to the CS2. Thus, notwithstanding the fact that the contiguity of the CS2 and the UCS was equal for both groups, there was a strong divergence in the intensity of the CR. These results not only show that contiguity is not sufficient for conditioning, they also seem to suggest that unique predictive information is an important determinant for conditioned responding to occur. Note that for the experimental but not for the control group, the CS2 was redundant in signalling the UCS. More recent evidence for the importance of predictive validity in conditioning is revealed by Dawson, Schell, and Banis (1986). They showed that conditioned (autonomic) responding only emerged if subjects perceived the CS as a predictor for shock outcome (but see Maltzman, 1987).

Another type of evidence for the suggestion that what is learned are propositional relations rather than responses can be obtained from sensory preconditioning experiments (e.g., Rescorla, 1974). In these experiments, 
animals are presented with paired presentations of two innoculous stimuli like pictures of circles and crosses. It should be stressed that no (conditioned) responding is evident in this phase of the experiment. In the second phase subjects receive repeated pairings of the CS1 with an UCS. In a third phase, the CS2 is tested and appears to elicit a CR. From the finding that the CS2 elicited conditioned responding without ever being paired with the UCS, it can be inferred that subjects silently learned the association between CS1 and CS2. A learning phenomenon that only became evident after the behavioural impact of the associated stimulus (CS1) was increased (i.e., inflated). The fact that the CS2 acquired excitatory properties as a result of a non-contingent inflation manipulation (that is, without changing the CS2/CS1 association) strongly suggests that apart from associations between the CS (i.e., CS2) and the UCS (i.e., CS1), also representations of the UCS are formed during paired presentations.

These and other observations have strongly influenced contemporary conditioning theories. These theories share the following two features: First, the content of learning pertains to CS-UCS contingency relations (but see Papini \& Bitterman, 1990); Second, the CS is thought to elicit a CR because of its association with the subjects' UCS representation. Both features may give rise to serious misrepresentations of the environment. That is, if perceived CS-UCS relations are indeed crucial for conditioned responses to occur, it may well be that subjects associate a CS with an UCS without objective contingencies given rise to this association. Especially in case subjects have strong a priori expectations they should be vulnerable for such biased covariation assessment (Alloy \& Tabachnik, 1984). As human expectations are not only derived from prior learning experiences, but can also be strongly influenced by symbolic information, it is clear that under some circumstances perceived contingencies may well deviate from true covariations. Similarily, the representation of the UCS may take an unrealistic format, as it is obvious that subjects' evaluation of the UCS can be affected by such different factors as coping style, isolated UCS experiences, symbolic information, and mood state (Davey, 1989).

\section{Contingencies, UCS representations, and Psychopathology}

The question arises, whether biased representations of contingencies might be a factor in the maintenance and exacerbation of psychopathological phenomena (e.g., depression, anxiety). Pertinent to this suggestion, Alloy and colleagues (for an overview, see Alloy, Abramson, \& Kossman, 1985) carried out a series of experiments that showed that the perception of contingencies is affected by mood state. Especially contingencies between responses and outcomes (i.e., controllability) were found to be prone to biased contingency perceptions. The basic outline of their experiments was as follows: depressed and non-depressed students were given a series of trials on which they either could decide to respond (i.e., pressing a button) or not to respond. Following 
this, a stimulus (e.g., a light) did or did not appear; the actual contingency between responses and outcomes was experimentally controlled. Subjects were instructed to find out to what extent the outcomes were related to their responses.

Overall, the findings (e.g., Alloy \& Abramson, 1979; see also Lennox et al., 1990) indicated that the contingency judgements of depressed subjects were highly accurate and independent of the valence of the outcome (i.e., positive vs. negative). In contrast, non-depressed subjects underestimated the contingency when the outcome was negative, and overestimated the contingency when the outcome was positive. It appears that non-depressed subjects possess a "positive cognitive set" that results in perceptions of contingencies that are more optimistic than is justified by the true contingencies. Further research of Alloy, Abramson, and Viscusi (1981) showed that this "optimistic bias" could be attenuated by the instatement of a depressive mood. Taken together, these data seem to suggest that depression is related to the absence of an optimistic bias rather than to the presence of a depressogenic bias. In other words, the absence of an illusion of control might be a vulnerability factor in the development of depression.

Employing a similar experimental procedure, Vázquez (1987; exp. 4) not only manipulated the valence of the outcomes, but also their reference (i.e., self-referent vs. other-referent). In line with the findings of Alloy and Abramson (1979), Vázquez showed that when the outcomes were positive self-referent, non-depressed students overestimated the contingency between their responses and the outcomes, while depressed subjects were quite accurate. However, in case the outcomes were negative self-referent, non-depressed students were quite accurate, whereas depressed students strongly overestimated the contingency between responses and outcomes. Thus, these results not only confirm the presence of an "optimistic bias" in non-depressed subjects, but also strongly suggest the existence of a "pessimistic bias" in depressed subjects. It seems reasonable to argue that both the presence of a "pessimistic bias" and the absence of an "optimistic bias" may act in way to confirm the negative mood state in depression.

In a similar vein it can be hypothesized that the information processing of fearful subjects is likewise biased in a way to confirm fear. From this perspective Tomarken, Mineka, and Cook (1989) argued that a bias to perceive illusory contingencies between phobia-relevant stimuli and aversive events would logically serve to maintain (or even enhance) phobic fear. To test the hypothesis that fearful subjects, indeed, are prone to overestimate the co-occurrence of feared stimuli and negative outcomes, Tomarken and colleagues designed an "illusory correlation" (IC) paradigm. In their experimental setup, subjects with and without a moderate fear of spiders (or snakes) were exposed to an extensive series of slides (CSs) comprising three different categories: Slides of spiders/snakes (fear-relevant), slides of flowers, and slides of mushrooms (neutral). Immediately at slide offset one of three outcomes (UCSs) occurred: a shock (aversive event), a tone (neutral), or nothing at all. Although all slide/outcome combinations occurred equally 
often, subjects typically overestimated the covariation between fear-relevant stimuli and aversive outcomes, i.e., reported an illusory correlation. This bias to overassociate fear-relevant stimuli and aversive consequences was particularly strong in the high fear group. The costs of such a judgment error seem obvious: Threatening characteristics of phobic stimuli are confirmed and in turn heightened threat might maintain and/or enhance phobic fear.

Not only perceived contingencies but also subjects' UCS representations are suggested to affect the strength of the conditioned response (e.g., phobic fear). This implies that all factors which can revalue the cognitive representation of the UCS, may have an impact on the intensity of fear. A recent experiment of White and Davey (1989) provide preliminary evidence to suggest that a non-contingent UCS-inflation procedure can, indeed, enhance conditioned responding in humans. The experiment of White and Davey consisted of three phases. During the initial phase some neutral visual stimuli $(C S+)$ were paired with a non-aversive tone (UCS), whereas other stimuli (CS-) were never followed by the UCS. In the second part of the experiment, half of the subjects (inflation group) were presented with a series of unsignalled UCSs that gradually became stronger in intensity and eventually reached an aversive level. The control group was exposed to UCSs that were kept constant at the same low level as during acquisition. During the final extinction phase, both groups were confronted with test presentations of the CS + and the CS-. Whereas differential electrodermal responding was absent during the initial acquisition phase, it did occur during the test stage, but only so in the inflation group.

The following case history may help to illustrate the potential clinical relevance of UCS revaluation processes in the etiology of anxiety disorders. Some person (L.L.) had always been mildly anxious in social situations (CS), and this mild anxiety was mostly accompanied by physical symptoms (UCS). The most salient symptom was intestinal unease. On one occasion, when L.L. was alone at home and not anxious at all, similar symptoms of intestinal unease led to an uncontrolable attack of diarrhoea (non-contingent UCSinflation). From that moment on L.L. catastrophically interpreted the symptoms of intestinal unease that he regularly experienced in company as a signal for losing control again. He became extremely anxious (CR) and developed severe agoraphobic symptoms.

\section{An Heuristic Model of Phobic Fear}

The findings presented above nicely fit with the conditioning model of acquired fears that was recently elaborated by Davey $(1989 ; 1992$; see also Figure 3). This model essentially discerns two different stages: The first stage concerns the associative strength between the CS and the UCS. During this stage, the predictive properties of the CS are evaluated (UCS expectancy). The second stage concerns the cognitive representation of the UCS. During this stage the cognitive UCS representation is evaluated. According to Davey's 
model, the CS (e.g., a dog) evokes a UCS representation (e.g., a dog's bite) as a result of associative processes, which, in turn, elicits the CR (e.g., fear). Note that the UCS representation is only evoked if the CS has sufficient predictive properties. The model implies that processes which influence the UCS expectancy and/or the UCS representation may also affect the intensity of the conditioned response.

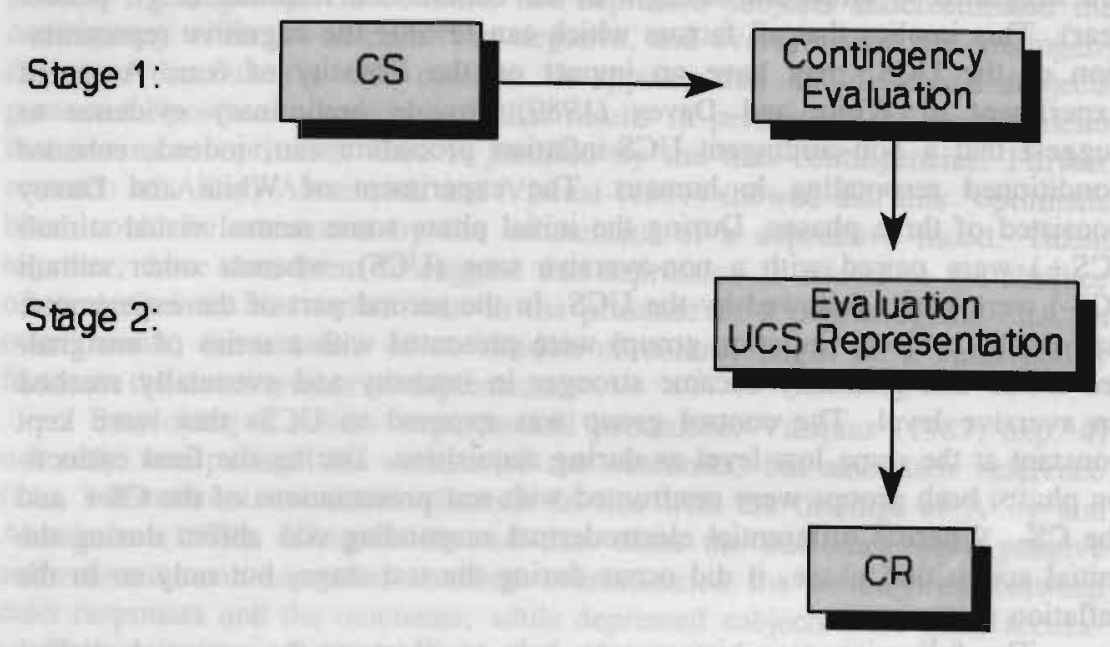

Figure 1.3: Schematic outline of Davey's (1989) conditioning model.

From this model several hypotheses can be derived that pertain to the following clinically relevant issues:

(1) Why does phobic fear not extinguish in the presence of overwhelming disconfirmatory evidence? That is, lifts only very seldom really crash, spiders do not tend to bite or strangle human subjects, and in the vast majority of cases palpitations are not followed by $a$ heart attack.

(2) How can it be explained that on the one hand a large part of subjects suffering from phobias can not recall any conditioning experience (e.g., Merckelbach, de Ruiter, van den Hout, \& Hoekstra, 1989) while, on the other hand a lot of non-phobic subjects do recall conditioning events but do not develop a phobia? (e.g., Merckelbach, Arrindell, Arntz, \& de Jong, 1992). Aren't such cases clearcut refutations of a conditioning interpretation of phobias? 
These two questions are the main focus of the present thesis. Pertinent to the first issue, Chapter 2 presents two experiments concerning the persistence of fear in the absence of contingent aversive events. More specifically, the experiments described in that Chapter test the hypothesis that phobic subjects show a bias to overassociate phobic cues and aversive outcomes (cf. Tomarken et al., 1989). In terms of the model presented in Figure 3, these experiments pertain to the question whether phobic fear can modulate the contingency evaluation of stage 1 , resulting in an illusory UCS expectancy. An additional issue that is addressed in Chapter 2 concerns the relationship between "covariation bias" and therapy success: Can covariation bias be reduced by behavioural therapy? Is the strength of covariation bias related to therapy outcome? Finally, the studies presented in Chapter 2 explore whether the covariation bias phenomenon is reflected in subjects' autonomic responding.

Chapter 3 is devoted to factors that may be responsible for biased (CSUCS) covariation detections in fearful subjects. From this perspective, section 3.a explores whether it is possible to induce an illusory correlation between a formerly neutral stimulus and an aversive outcome by means of a conditioning-like procedure. This section also addresses the important question whether such an illusory correlation once induced is self-supporting and resistant to extinction procedures. The sections 3.b and 3.c concern the potential importance of a priori expectations and biased encoding in the generation of fear-confirming illusory correlations. Section 3.d contains two studies that can be considered as preliminary attempts to pinpoint covariation bias to neuropsychological mechanisms. The first study aims to replicate the previously reported finding of Merckelbach, Muris, and de Jong (1990) that in normals there is a positive correlation between a right hemispheric thinking style and anxiety scores. The second study tentatively explores whether covariation bias is a function of the right hemisphere and whether covariation bias may be a mediating factor in the relationship between anxiety and hemisphericity.

A major problem with studies on the relationship between cognitive bias and phobic fear pertains to the status of the bias: Is the bias a causal factor or merely an epiphemomenon of phobic fear? That is, even if covariation bias is only evident in high fear subjects and even if the bias disappears once anxiety is successfully treated, this, of course, does not preclude the possibility that anxiety evokes the bias rather than vice versa (cf., Merckelbach, van Hout, De Jong, van den Hout, 1990). The study described in Chapter 4 is an empirical test of the hypothesis that overestimation of threat plays a causal role in the origins and maintenance of irrational fears (e.g., Hawton, Salkovskis, Kirk, \& Clark, 1989).

Pertinent to the second issue, Chapter 5 presents a series of experiments that investigates whether non-contingent manipulation of the UCS representation affects conditioned responding in humans. If subjects are susceptible to postconditioning UCS devaluation, this could explain why some subjects who 
do not develop a phobia, do recall conditioning experiences. In a similar vein, if it turns out that UCS inflation is a robust phenomenon in the context of human conditioning, this might explain the finding that a considerable number of phobic subjects can not recall any conditioning experience. To investigate further the latter possibility, the studies in section 5.a concentrate on the UCS inflation phenomenon in human "fear" conditioning. These experiments can be considered as attempts to cross-validate and extend the previous findings of White and Davey (1989). In terms of the model presented in Figure 3, these experiments focus on the second stage and test whether isolated UCS experiences can modulate the evaluation of UCS representations.

It seems reasonable to suggest that subjects' personality characteristics interact with UCS inflation scenarios. Pertinent to this suggestion, Eysenck (1976) argued that a valid theory on human conditioning should incorporate both situational factors and personality characteristics. From this perspective, section 5.b explores the possibility that UCS revaluation processes are affected by subjects' coping style.

\section{A New Index of Phobic Fear}

In the preceding paragraphs it has been suggested that biased contingency perceptions and biased cognitive UCS representations are important factors in the etiology and maintenance of phobic fear. However, it should be noted that from the heuristic model presented in Figure 3, no clear predictions can be inferred as to what extent UCS expectancies and UCS representations are related to subjects' evaluation of the phobic stimulus. Of course, one may infer the CS evaluation from subjects' skin conductance responses (SCRs) to the phobic stimuli (e.g., Chapter 2). Yet, it should be acknowledged that SCRs are not very specific and can perhaps be best conceptualised as an index of arousal rather than as a measure of hedonic valence (Lang, Bradley, \& Cuthbert, 1990; Hamm \& Stark, 1993). Another strategy would be to just ask subjects to rate the affective valence of the CS (e.g., Chapter 5). Unfortunately, such a strategy is susceptible to distortion, as it demands reflection, needs some explanation, and is vulnerable to demand (e.g., Nisbett \& Wilson, 1977). Recent research of Lang and colleagues suggests an appropriate alternative index of emotional valence: The startle probe response.

In a series of experiments (for a review see Lang, Bradley, \& Cuthbert, 1990), Lang and colleagues showed that the startle response is modulated by emotional state. That is, positive foreground stimuli (e.g., slides depicting nudes) appear to inhibit the response, whereas the startle probe response is potentiated during negative foreground stimuli (e.g., slides of spiders). In contrast, concurrently monitored SCRs did not differentiate between positive and negative slides, whereas the SCRs to neutral slides were relatively small. Thus, whereas startle magnitudes show a linear increase from positive to negative slides, the relationship between arousal and slide valence appears to reflect a quadratic (U-shaped) function. These results seem to indicate that 
affect-induced startle modulation is independent of arousal (see also Hamm \& Stark, 1993). The recent finding of Hamm and colleagues that startle responses increase as a result of aversive classical conditioning, provides additional support for the idea that the startle probe response would be a useful index of phobic fear (Hamm, Greenwald, Bradley, Cuthbert, \& Lang, 1991).

Taken together, the available data sustain the suggestion of Vrana, Spence, and Lang (1988) that "the startle probe would be useful in evaluating treatment methods and in the prognostic assessments of patients with pathological anxiety" (p.491). Following this, Chapter 6 presents two studies that test the evaluative and prognostic properties of the startle probe response in the treatment of spider phobia. In addition, section 6.b pays special attention to the relationship between affect and arousal, to test the proposed specificity of the startle probe response in a clinical context. 


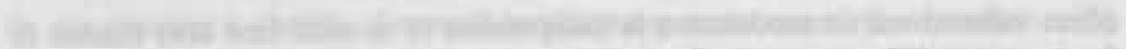

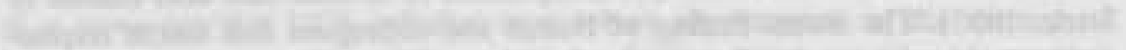

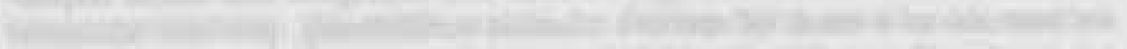

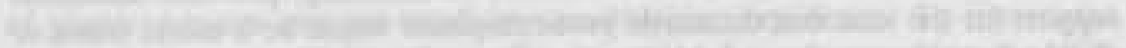

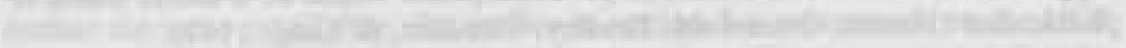
-

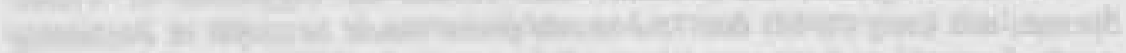
1.1.

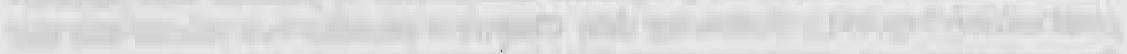
ant ant

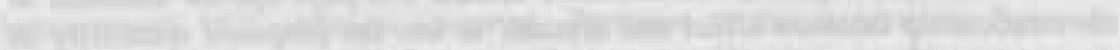
7.

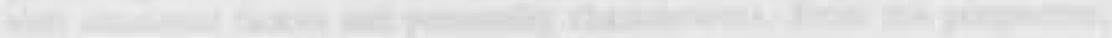

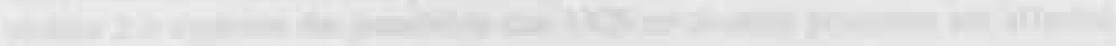

10

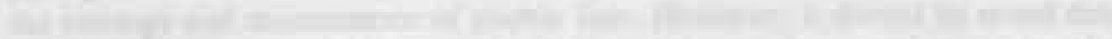

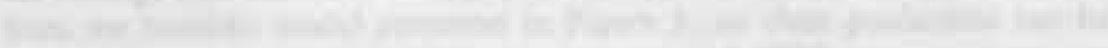

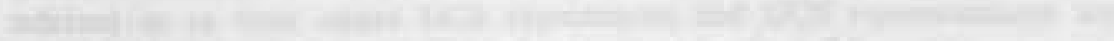

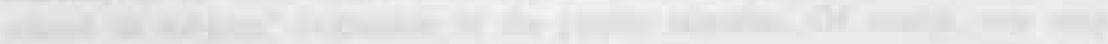

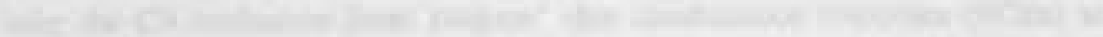
-

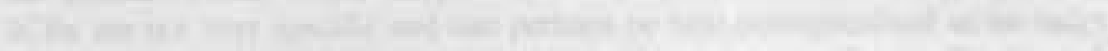

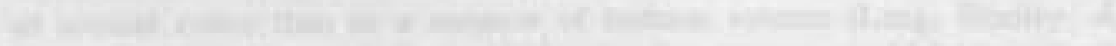

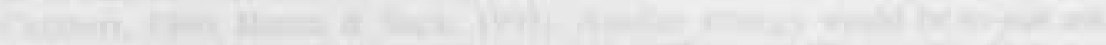

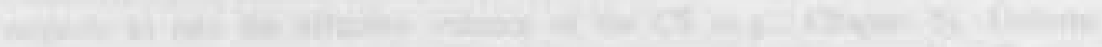

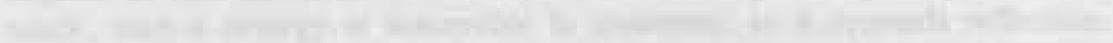

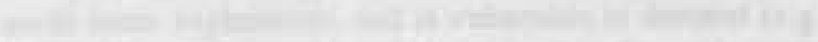

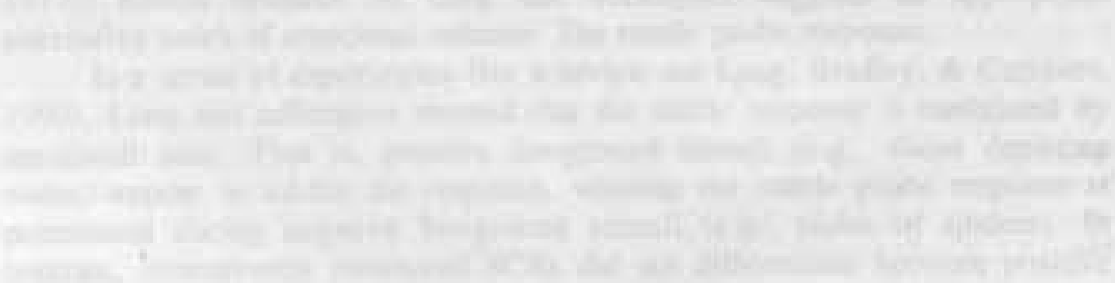

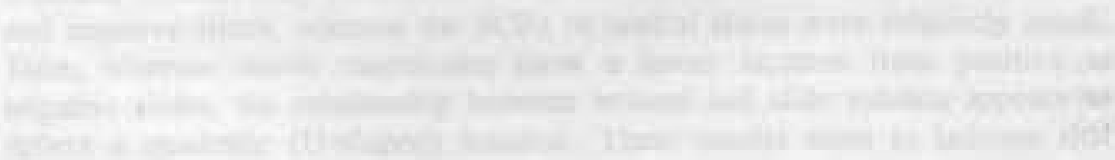




\section{2.a THE PHENOMENA I: A FIRST REPLICATION}

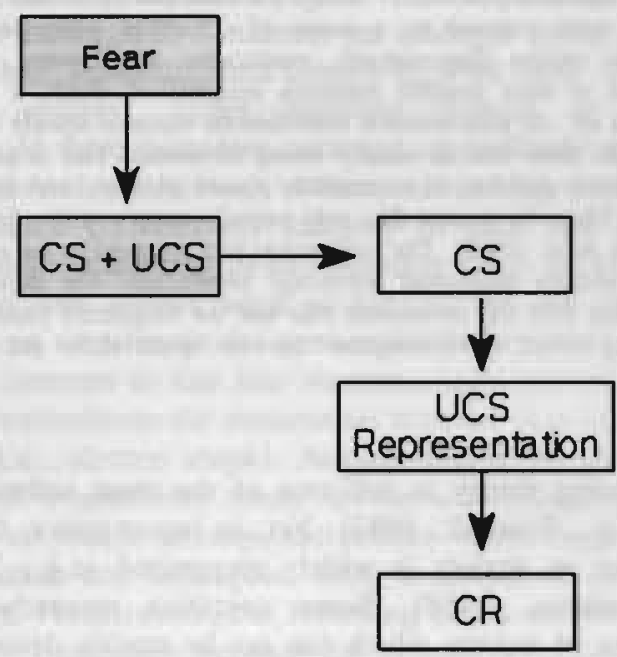

This chapter is partially based on a paper presented at the 20th European Congress of Behavioural Therapy in Paris (1990). It is published as: De Jong. P.J., \& Merckelbach, H. (1991). Covariation bias and electrodermal responding in spider phobics before and after behavioural treatment. Behaviour Research and Therapy, 29, 307-314.

Acknowledgements: The authors would like to thank the therapists Edith Lavy. Germie van den Berg, and Amoud Arntz. In addition, we are grateful to Dorien Wolfs who took care of the organizational aspects of this study. Furthermore, we would like to thank Dr. Karel Eisses who kindly provided Drosophilae for our spiders. 


\title{
Covariation Bias and Electrodermal Responding in Spider Phobics Before and After Behavioural Treatment
}

\begin{abstract}
The present study investigated whether a covariation bias is present in severe spider phobics and whether such bias is modified by successful treatment. In addition, this study sought to examine whether a covariation bias is linked to differential autonomic responding. Subjects were 20 untreated phobics, 19 treated phobics, and 18 no-fear controls. Subjects were exposed to a series of 72 slides comprising three categories: spiders (fear-relevant), mushrooms, and flowers. At slide offset one of three possible outcomes occurred: A shock, a tone, or nothing at all. All slide-outcome combinations occurred equally frequent. The results show that an equally strong covariation bias is present in severe spider phobics, in successfully treated phobics, and in no-fear controls. Thus, the present data only partially sustain earlier findings of Tomarken et al. (1989). The covariation bias appeared to be mimicked by differentially heightened autonomic responding. The current data suggest that both the covariation bias and the heightened physiological responding reflect a "beloningness" between spider slides and aversive outcome.
\end{abstract}

Classical conditioning theory is still one of the most influential theories on phobic anxiety (e.g., Eysenck, 1987). Yet, in recent years, the importance of cognitive processes in anxiety is widely recognized (e.g., Williams, Watts, MacLeod \& Mathews, 1988). Some cognitive researchers have drawn attention to aspects of anxiety which can not be readily described in terms of classical conditioning, like for instance, the attentional bias phenomenon (Mathews \& Macleod, 1986). Other researchers in this field have performed studies which are located on the very borderline of cognitive theory and classical conditioning. A prominent example of this type of research are studies concerned with the variables that influence human inferences. Most relevant to the present context is research on the ability to detect covariations between events (see Alloy \& Tabachnik, 1984, for an extensive review on this topic).

Information about covariations of events provides people with a means to explain the past, control the present, and predict the future. Such information is important in order to optimalize the likelihood of obtaining desired outcomes and avoiding aversive ones. From a learning theoretical point of view, the phobic stimulus may be considered as a danger signal (Reiss, 1980) or, in other words as a predictor of the occurrence of an aversive UCS (e.g., Eelen, 1982). If phobics, indeed, perceive the phobic stimulus as a predictor of something terrifying about to occur (and there is some tentative evidence that they do so; see Arntz et al., 1990), it is not at all surprising that phobics tend to avoid this phobic stimulus. However, it is obvious that such a sequential relationship between phobic stimulus and aversive outcome is non-existent; 
This leaves us with the intriguing question of why phobics persistently act in a way as if such a relationship does exist in real world.

From this perspective, Mineka and Tomarken (1989) hypothesized the presence of a covariation bias in clinical anxious subjects. Such bias would result in an overestimation of the contingency between feared objects and aversive events. Such bias in information processing would appear to be a particularly direct and powerful way to confirm or enhance fear. In order to investigate whether phobic subjects, indeed, overestimate the covariation between fear-relevant stimuli and aversive outcomes, Tomarken $e t$ al. (1989) performed a series of experiments using an "illusory correlation" paradigm. In their studies, low and high fear students (an analogue group) were exposed to a series of 72 slides. Three different categories were used: fear-relevant slides (e.g., depicting spiders), slides depicting flowers or slides depicting mushrooms. One of three outcomes occurred immediately at slide offset: An electrical shock (aversive outcome), a tone, or nothing at all. There was no systematic correlation between any category of slide and type of outcome. Thus, the conditional probability of any outcome given the prior occurrence of any slide category was always equal to $1 / 3$. At the end of the experiment, subjects were asked to estimate the covariation between all stimulus/outcome combinations. In contrast to low fear students, high fear students were found to dramatically overestimate the covariation between fear-relevant stimuli and aversive events (i.e., electric shock). Acknowledging the potential importance of Tomarken et al.'s laboratory model for the understanding of the aetiology and maintenance of phobic anxiety, the present study sought to replicate the findings of Tomarken and colleagues, using a sample of clinical (rather than analogue) phobics. Furthermore, the following issues were examined: Firstly, is there a positive correlation between the severity of phobic complaints and the strength of covariation bias? Secondly, does the covariation bias shown by phobics diminish or even disappear after successful treatment? Finally, is the covariation bias related to autonomic "conditioned" responding. Note in this respect, that a number of studies suggest that human covariation judgement is sensitive to the same factors as conditioned responding (e.g., Dickinson \& Shanks, 1985; Lovibond, 1988). In addition, it has been shown that an experimentally induced "illusory correlation" is associated with differential autonomic responding in normals (de Jong, Merckelbach \& Arntz, 1990). The present study was conducted along the lines of the "illusory correlation" paradigm employed by Tomarken et al. (1989). In contrast to Tomarken et al. (1989), however, this study relied on a group of severe spider phobics, 20 before and 19 after behavioural treatment. To evaluate whether the $a$ posteriori reported covariation estimates were linked to differential physiological responding, skin conductance responses were measured as an index of autonomic responding. 


\section{Method}

\section{Subjects}

Subjects were 42 female spider phobics (mean age 30.1, range: 18-55) and 18 no-fear controls matched for sex and educational level (mean age 26.3, range: 20-48). Phobic subjects were recruited through articles about spider phobia treatment in regional newspapers and by a program on satellite television. Phobic subjects were invited to participate in research in return for "free" treatment (see below). One group of phobic subjects $(n=23$; "before treatment group") was tested before treatment, while a second group of phobic subjects $(n=19$; "after treatment group") was tested after treatment. The no-fear controls were recruited through advertisements in regional newspapers and were paid for their participation in this experiment. The mean score on the Spider fear Questionnaire (SPQ; Klorman, Weerts, Hastings, Melamed \& Lang, 1974) for the before-treatment, after-treatment, and non-phobic group was 23,12 , and 3 , respectively. It should be stressed that the mean SPQ before treatment was similar for both phobic groups $[t(40)<1]$, while the "after treatment" group dramatically improved by therapy as indexed by SPQ scores $[t(18)=9.78, p<0.001]$. Note that the SPQ score of the phobic group is comparable to the mean score that Fredrikson (1983) reported for his phobic sample. During the experimental exposure to slides of spiders (see below), three subjects in the "before treatment" group panicked. These subjects preferred to withdraw from the experiment. The data of these subjects were excluded from the final analyses, leaving 20 subjects in the "before treatment" group.

\section{Apparatus and Stimulus Materials}

Three categories of stimuli were used throughout the experiment: 4 different slides depicting spiders, 4 different slides depicting mushrooms, and 4 different slides depicting flowers (e.g., Ohman, Fredrikson, Hugdahl \& Rimmo, 1976). Slides were projected by a Kodak Carousel onto a screen ( 80 $x 120 \mathrm{~cm}$ ), approx. $2 \mathrm{~m}$ in front of the subject. Three types of outcomes occurred during the experiment: a 2-s shock, a 2-s tone, or nothing at all. Shocks $(\mathrm{dc})$ were delivered from a specially designed apparatus (maximum capacity $40 \mathrm{~mA}$ ) and administered to the subjects lateral side of the upper (right) arm through two electrodes $(8 \mathrm{~mm}$ diameter $\mathrm{Ag}-\mathrm{AgCl}$ ). Tones were delivered by a standard tone generator $(50 \mathrm{db})$.

During the experiment, electrodermal activity was recorded from two Beckman $\mathrm{Ag}-\mathrm{AgCl}$ electrodes (8 $\mathrm{mm}$ diameter), placed on the medial phalanges of the second and third finger of the non-dominant hand. The electrodes were filled with an isotonic paste and connected to a Beckman Skin Conductance Coupler (type 9844). Frequency and depth of ventilation was recorded by means of a Beckman respiratory belt connected to a Beckman 
Voltage/Pressure/Pulse Coupler. Physiological signals were recorded on a Beckman Polygraph. Stimulus presentation, tones, shocks, and intertrial intervals were controlled by a PDP Minc-II computer.

\section{Procedure}

Phobic subjects were randomly assigned to one of the two groups (before vs. after treatment). Half of the phobic subjects first underwent a $2,5 \mathrm{~h}$ onesession therapy as described by Ost (1989) before they participated in the experiment proper. The one-session treatment consists of exposure in vivo and modeling and has been found to yield good immediate and long-term results (Ost, 1989; Merckelbach, de Jong \& Arntz, 1990). The "before treatment" subjects first participated in the experiment and then underwent behavioural treatment.

After introduction to the laboratory, electrodes and respiratory belt were attached to the subject. Next, a shock work-up procedure was carried out in order to set the intensity level of the shock. Electrical current was increased in steps of $0.2 \mathrm{~mA}$ until the subject indicated that the shock was uncomfortable but not painful. Following this, the subject was informed about the experimental task. It was explained that she had to determine whether or not there was a relationship between a particular slide type and a particular type of outcome. Subjects were exposed to a series of 72 slides of $8 \mathrm{~s}$ duration each. Three different categories were used: Spiders (fear-relevant stimuli), mushrooms, and flowers (neutral stimuli). One of three possible outcomes occurred at slide offset: a 2-s shock (aversive outcome), a 2-s tone, or nothing at all (neutral outcomes). Across all trials, the conditional probability of any outcome given the prior occurrence of any category of slide was $33 \%$ and so was the probability of occurrence of each category of slide and each type of outcome. Stimulus-outcome combinations were randomly distributed across trials with the restriction that on adjacent trials no identical stimulus-outcome combinations occurred. Three different series were used. At the end of the experiment, subjects completed the Probability Questionnaire (PQ). The PQ comprised three sections. Questions in the first section ask subjects to estimate the percentage of occurrence of each outcome given the prior occurrence of each slide type. The second section asks subjects to estimate the percentage of times each slide type occurred given the occurrence of each outcome. Finally, subjects were asked to estimate the base rates of each category of slides and each type of outcome during the 72 trials. For all estimates visual analogue scales (VASs) were used ranging from $0 \%$ to $100 \%$.

\section{Data Analysis and Reduction}

$P Q$. The a posteriori reported covariation estimates were subjected to 3 (group) X 3 (stimulus) X 3 (outcome) analyses of variance (ANOVA), with 
the last two factors being within-subjects factors. Base-rate estimates of stimuli and outcomes were analyzed by means of 3 (group) X 3 (outcome/stimulus) ANOVAs, with the last factor being a within-subjects factor.

Skin Conductance. Three different skin conductance components were scored (Prokasy \& Kumpfer, 1973): The first interval response (FIR; 1-4s after CS onset, for each trial), the third interval omission response (TOR; $1-4 \mathrm{~s}$ after CS offset, on non-shocked trials), and the unconditioned response (UCR, on shocked trials). The FIR is said to reflect an orienting response (e.g., Stern \& Walrath, 1977), whereas the TOR is thought to reflect "suprise" about the non-occurrence of shock (e.g., Seligman, Maier \& Solomon, 1971). The UCR was considered as an index of shock impact. It was anticipated that the "before treatment" phobics would react with stronger FIRs to spider slides than "after treatment" phobics or normal controls. It was also anticipated that the covariation bias would be paralleled by heightened TORs after spider slides. Following the criteria of Stern, Ray, and Davis (1980), responses associated with ventilatory irregularities were omitted. SCR data were analyzed as magnitudes (square root transformed) and probabilities. Missing responses were replaced by estimates based on two adjacent trials. The electrodermal data were subjected to 3 (group) $\times 3$ (stimulus) $\times 4$ (block of trials) ANOVAs, with the last factors being within-subjects factors.

\section{Results}

$P Q$. Figure 2.1 depicts the most relevant covariation estimates revealed from the first section of the PQ [P(outcome/slide)].

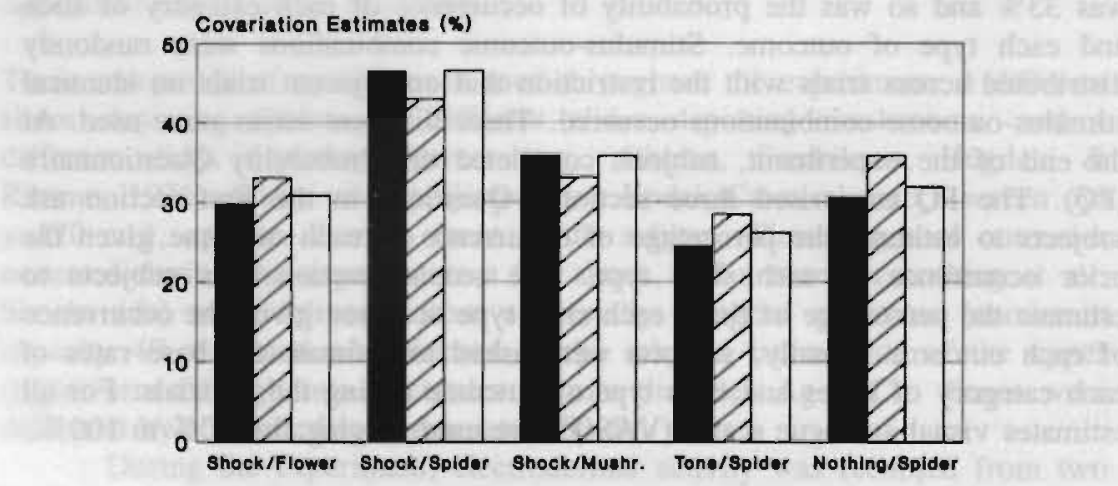

Betore Treatment

After Treatment

Control Group

Figure 2.1: Most relevant a posteriori reported probability estimates of each outcome given the prior occurrence of each slide type [P(outcome/slide)]. Note that the conditional probability for all combinations was in fact 1/3. 
A 3 (group) $\times 3$ (stimulus) $\times 3$ (outcome) ANOVA showed an interaction of stimulus and outcome which can be attributed to an overestimation of the spider-shock combination in all groups $[F(4,216)=13.61, p<0.001]$. In contrast to what was anticipated, no interaction with group appeared $[F(8,216)<1.0]$. The ANOVA performed on the PQ scores of the second section [P(slide/outcome)] revealed similar results. That is, an interaction of stimulus and outcome appeared $[F(4,216)=6.39, p<0.001]$ that was not modulated by group $[F(4,216)<1.0]$. Likewise, no significant Pearson p-m correlations between SPQ and spider-shock covariation estimates were found. No significant differences in base rate estimates appeared.

SCR. As both probability and magnitude indices of SCR revealed identical results, only magnitudes are reported here. Figure 2.2 shows the FIRs. A 3 (group) $\times 3$ (slide) $\times 4$ (block of 6 trials) ANOVA revealed a main effect of trial (due to habituation) $[F(5,270)=40.61, p<0.001]$. Also, a main effect of stimulus was found indicating that, overall, responses were stronger on spider trials than on neutral trials $[F(2,108)=27.45, p<0.001]$. In addition, the "before treatment" phobics gave the largest responses to spider slides, as was confirmed by the significant interaction of group and stimulus $[F(4,108)=3.45, p<0.05]$. No group $\times$ stimulus $\mathrm{x}$ trial interaction was found $[F(20,540)<1.0]$. Thus, no evidence was obtained for a differential habituation pattern over spider trials between groups.
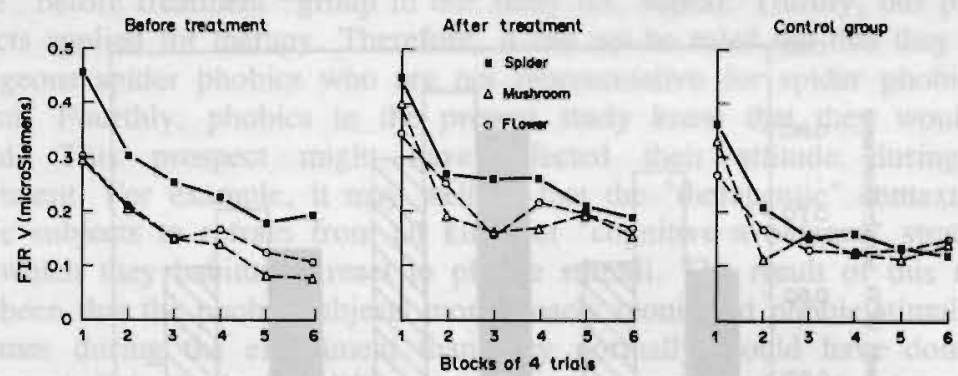

Figure 2.2: The first interval skin conductance responses (FIRs) on spider, flower, and mushroom trials for all groups. Data presented in blocks of 4 trials.

Figure 2.3 depicts the TORs. A 3 (group) $\times 3$ (slide) $\times 4$ (block of 2 trials) ANOVA yielded, again, main effects of trials (habituation) $[F(3,162)=15.73$, $p<0.001]$ and stimulus $[F(2,108)=5.82, p<0.01]$. The latter effect indicates that, overall, shock was more frequently expected after a spider slide than after a neutral slide. Yet, like the PQ and FIR data, no interaction with group emerged. Finally, a 3 (group) $\times 3$ (stimulus) ANOVA performed on the UCR data showed a main effect of stimulus. As can be seen in figure 2.4 , this can 
be attributed to greater UCRs on the fear-relevant trials across groups $[F(2,108)=18.16, p<0.001]$. There was no interaction of stimulus with group $[F(4,108)<1.0]$.

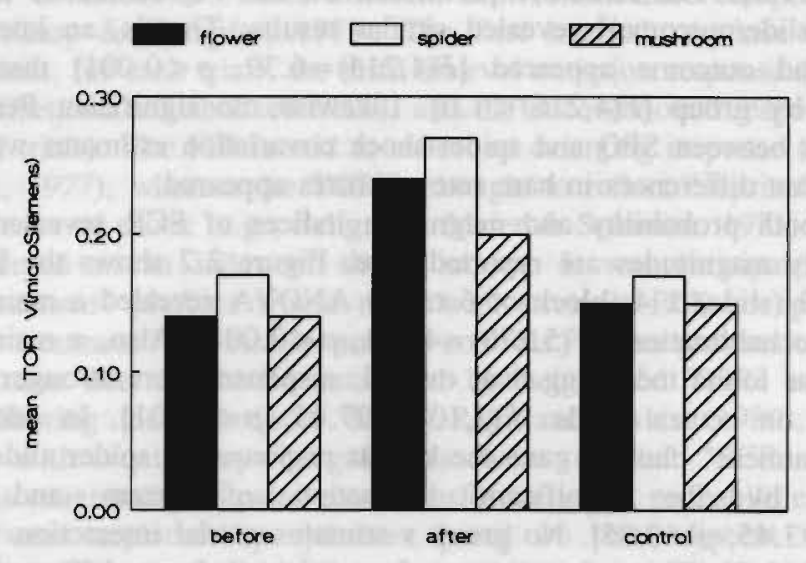

Figure 2.3: Mean third interval omission responses (TORs) on spider, flower, and mushroom trials for all groups.

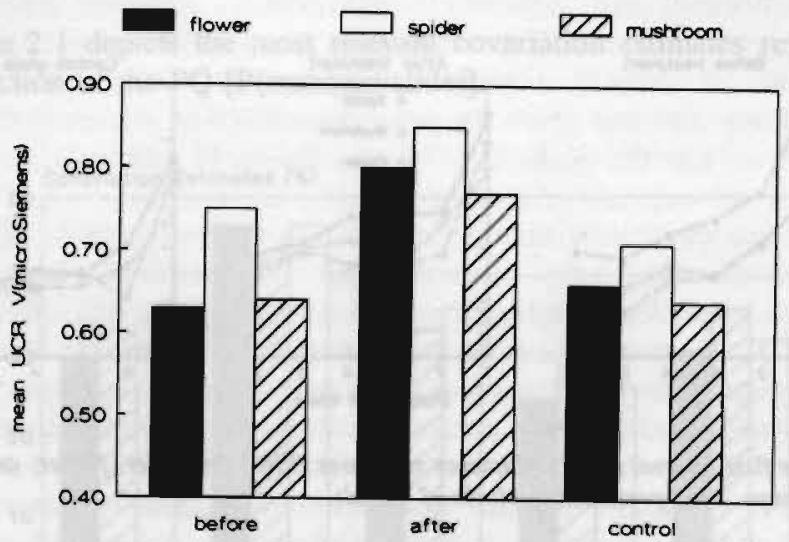

Figure 2.4: Mean unconditioned skin conductance responses (UCRs) on shocked spider, flower, and mushroom trials for "before treatment" phobics, "after treatment" phobics, and no-fear control subjects. Data are presented in blocks of 2 trials.

\section{Discussion}

The present results sustain the conclusions previously drawn by Tomarken et 
al. (1989) only partially. On the one hand, spider phobics were, indeed, found to overestimate spider-shock combinations. This overestimation was evident both when subjects estimated the percentage of occurrence of each outcome given the prior presentation of each slide type [P(outcome/slide)] and when subjects estimated the percentage of times each slide type was presented immediately before the occurrence of each outcome [P(slide/outcome)]. On the other hand, data clearly showed that the covariation bias was not restricted to "before treatment" phobics. As a matter of fact, all groups showed a strong covariation bias. The fact that there were no significant Pearson correlations between SPQ scores and covariation bias indices underlines the non-specificity of the covariation bias phenomenon. In passing it should be noted that a similar lack of correlation between covariation bias and self-reported fear was reported by Merckelbach, van den Hout, and de Jong (1990) in their study of illusory correlations in normal subjects.

The apparent discrepancy between the current results and those presented in Tomarken et al. (1989) is hard to explain. A comparison between covariation estimates reported by Tomarken et al. and those found in the present study suggests that high fear subjects in the Tomarken et al. study had a stronger covariation bias than the "before treatment" phobics in the present study. Are there reasons to believe that the samples used by Tomarken et al. differ from those used in the present study? Firstly, whereas Tomarken et al. (1989) worked with undergraduates, subjects in the present study were older and less educated. Secondly, the Tomarken et al. study relied on analogue phobics, whereas the present study relied on clinical phobics: The high fear subjects of Tomarken et al. had a mean score of 15 which is well below that of the "before treatment" group in our study (cf. supra). Thirdly, our phobic subjects applied for therapy. Therefore, it can not be ruled out that they were courageous spider phobics who are not representative for spider phobics in general. Fourthly, phobics in the present study knew that they would be treated. This prospect might have affected their attitude during the experiment. For example, it may well be that the "therapeutic" context lead phobic subjects to refrain from all kinds of "cognitive avoidance" strategies with which they habitually react to phobic stimuli. The result of this might have been that the phobic subjects more closely monitored phobic stimuli and outcomes during the experiment than they normally would have done. In Alloy and Tabachnik (1984)'s terms, the therapeutic context might have stimulated phobic subjects to base their covariation estimates not only on $a$ priori expectations (that would dominate in a non-therapeutic context) but also on situational information. Currently, we are preparing a study that addresses the effect of context (therapeutic vs. non-therapeutic) on phobic's covariation bias.

The revealed covariation bias across groups was associated with heightened autonomic responding as indexed by the TOR, FIR, and UCR. In this respect the present results fit nicely with an earlier study which showed that experimentally induced "illusory correlations" are associated with autonomic responding (de Jong et al., in press). 
Both physiological and subjective data indicate that subjects, irrespective of their being phobic or not, overestimate the contingency between spiders (i.e., fear-relevant cue) and shock (i.e., aversive outcome). The greater FIRs, TORs, and especially the greater UCRs on the fear-relevant trials can be attributed to a match ("belongingness") between the primary affective response organization of the stimulus and that of the outcome (e.g., Lang, 1985). Germane to this issue is a study on fear conditioning and "belongingness" of Hamm, Vaitl, and Lang (1989) in which it was shown that a relatively high $a$ priori rated belongingness between certain stimuli and outcomes enhances conditionability of autonomic responses to those stimuli. From this perspective, the greater physiological responsivity (especially the UCRs) on fear-relevant trials found in the present study, may be seen as a reflection of "belongingness" between spider slides and electric shock (see also Cook, Hodes \& Lang, 1987). In a similar vein it can be argued that the revealed covariation bias was mediated by "belongingness". Although the concept of "belongingness" has frequently been used interchangeably with the concept of "preparedness" (e.g., Ohman et al., 1976), it should be noted that both concepts differ in important respects. Whereas "preparedness" refers to a genetically based readiness to react with fear responses to such stimuli as spiders and snakes, "belongingness" is a much broader concept that does not preclude non-biological mechanisms. For example, Grant (1968) found that the eye-blink response of human subjects can be better conditioned to a slide depicting "blink" than to slide depicting "don't blink". In this case it is obvious, the "belongingness" between the eye-blink response and the blink stimulus is of non-biological nature.

In summary, the current data show 1 that a covariation bias is present in severe spider phobics as well as in successfully treated spider phobics and normal controls; 2 that this covariation bias is associated with heightened autonomic responding. It seems plausible to assume that both covariaton bias and heightened physiogical responding reflect a "belongingness" between fearrelevant stimuli and aversive outcome. 


\section{2.b THE PHENOMENON I: $A$ SECOND REPLICATION}

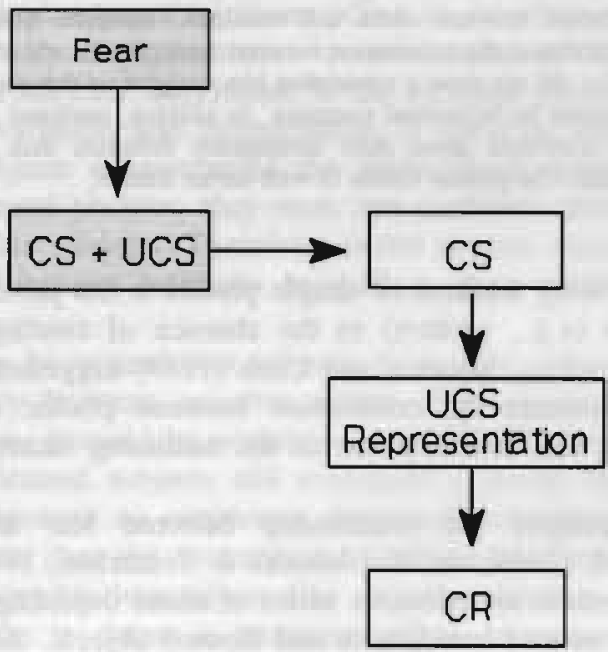

This chapter is based on papers presented at the First International Congress on Stress, Anxiety, and Emotional Disorders (Braga, 1991) and die 33.Tagung der experimentell arbeitender Psychologen (Giessen, 1991). Parts of this chapter are published as: De Jong, P.J., Merckelbach, H., \& Arntz, A. (1993). Covariation detection in treated and untreated spider phobics. Journal of Abnormal Psychology, 101, 724-727.

Acknowledgements: We thank the therapists Germie van den Berg. Edith Lavy, Miranda Lomme, Anja Meijboom, and Dorien Wolfs. In addition, we are grateful to Dr. Karel Eisses, who kindly provided Drosophila for our spiders. Furthermore, we are grateful to Henk Nijman, Gerrie van Wunnik and Theo van Aerts for their technical assistance. 


\title{
Covariation Detection in Treated and Untreated Spider Phobics
}

\begin{abstract}
Twenty treated and 18 untreated spider phobics were exposed to a series of 72 slides. Three different categories of slides were used: phobiarelevant slides (spiders), alternative fear-relevant slides (weapons), and neutral slides (flowers). Slides were randomly paired with either a shock, a tone, or nothing at all. Despite the absence of a systematic correlation between slides and outcomes, untreated phobics strongly overestimated the covariation between spider slides and shock. Treated phobics did not show a covariation bias, suggesting that such bias can be modulated by behavioral treatment. In addition, untreated subjects were more confident about their contingency estimates than were treated subjects. The present results fit with earlier studies.
\end{abstract}

One of the striking features of simple phobia is the persistence of anxiety for certain stimuli (e.g., spiders) in the absence of contingent aversive events. Recently, Tomarken, Mineka, and Cook (1989) suggested that phobic subjects tend to overestimate the covariation between phobic stimuli and aversive events. Such a bias could be one of the mediating factors in the maintenance of phobic fear.

To investigate the relationship between fear and covariation bias, Tomarken et al. (1989; see also Mineka \& Tomarken, 1989) exposed high and low fear students to an extensive series of slides depicting fear-relevant (spider or snake) and neutral (mushroom and flower) objects. All slides were equally frequently followed by aversive (i.e., electric shock) and (i.e., nothing/tones) neutral outcomes. Despite the fact that all slide-outcome combinations occurred equally frequently, Tomarken et al. (1989) found that high fear students in contrast to low fear students systematically overestimated the contingency between fear-relevant slides and electric shock. The authors argued that this "illusory correlation" is a manifestation of a more general bias in anxious subjects to associate fear-relevant stimuli with aversive events. They concluded that such covariation bias might play a critical role in the maintenance of phobic fear.

To further explore this hypothesis, de Jong and Merckelbach (1991) employed the same experimental procedure using untreated and treated women who were spider phobics. From Tomarken et al.'s study it was inferred that untreated but not treated spider phobics would show a covariation bias. However, that study only partially sustained the earlier findings of Tomarken and colleagues. That is, untreated spider phobics, indeed, significantly overestimated the spider/shock contingency, but quite unexpectedly, successfully treated phobics likewise showed a covariation bias (and to a similar degree). Thus, although spider fear was dramatically reduced as a result of a one-session in vivo exposure treatment (Öst 1989), the covariation bias of 
treated subjects appeared to be similar to that of untreated subjects. To some extent, the discrepancy between the findings reported by Tomarken et al. and those reported by de Jong and Merckelbach might be due to sample differences. Note that Tomarken et al. worked with undergraduates, whereas subjects in the de Jong and Merckelbach study were older and less educated. Furthermore, the fear level of treated subjects in this study was substantially higher than that of the low-fear subjects in the Tomarken et al. study. The residual fear of treated subjects might have been sufficient to induce a considerable covariation bias.

The present study was undertaken to investigate two additional explanations for the apparent absence of treatment effect on covariation bias in spider phobics. First, not only the quantitative overestimation of the spider/shock contingency but also subjects' confidence in the reported covariations may be important to the covariation bias phenomenon. It could be hypothesized that although treated subjects overestimated the spider/shock contingency to the same extent as untreated phobics, they were less confident about their estimates than were untreated subjects. Therefore, in the present study subjects were asked to report contingency estimates as well as their confidence in these estimates.

Second, it can be argued that subjects in general selectively overestimate the contingency between aversive events and salient danger stimuli. From this perspective, it could be reasoned that in the de Jong and Merckelbach (1991) study, treated subjects still evidenced a strong bias to associate spiders with shock because no concurrent danger-related stimuli were available. This would mean that the occurrence of additional danger stimuli may render the spider stimulus less salient (for treated subjects), which in turn makes it more likely to detect a treatment effect on covariation bias, if present. For that reason, alternative danger-related stimuli were included in the present study. More specifically, slides of aimed weapons were used instead of slides depicting mushrooms.

It seems reasonable to argue that subjects in general tend to associate shock with those stimuli that have the potency to cause aversive events. Therefore, adding weapons as a third category results in a sequence of slides that can be considered as even more ambiguous as to the stimulus/shock contingencies than the sequences used so far (comprising flowers, mushrooms, and spiders). The perception of contingency can be described as a function of a priori expectation and current situational information. The more ambiguous the situational information, the more subjects rely on a priori expectations (e.g., Alloy \& Tabachnik, 1984). Obviously, before treatment, spiders are more closely associated with aversive events than both weapons and flowers. Predominantly relying on a priori expectations would, therefore, result in (untreated) phobics specifically associating spiders with shock (despite the inclusion of weapon slides). One expects that successful treatment will reduce the strength of the a priori spider-danger connection to a degree that is roughly comparable to the strength of the $a$ priori weapon-danger connection. Therefore, it was anticipated that the covariation bias would be attenuated or 
even absent in treated spider phobics. Moreover, treated subjects were expected to judge the covariations with lower confidence than were untreated spider phobics. As in the previous study, electrodermal activity was measured in an attempt to replicate the earlier finding that covariation bias is associated with differential autonomic responding.

\section{Method}

\section{Subjects}

Subjects were 38 women who were spider phobics (mean age $=29.6$ years, range $=17-61$ years). They applied for treatment after reading articles in regional and national newspapers about an earlier "spider-project" at Limburg University. Phobic subjects were invited to participate in research in return for "free" treatment. One group of subjects $(n=18)$ was tested before treatment, and a second group of subjects $(\mathrm{n}=20)$ was tested after treatment. All subjects met criteria from the Diagnostic and Statistical Manual of Mental Disorders (rev. 3rd ed.; American Psychiatric Association, 1987) for simple phobia. The mean scores on the Spider Phobia Questionnaire (SPQ; Klorman, R., Weerts, T.C., Hastings, J.E., Melamed, B.G., \& Lang, P.J., 1974) for the untreated and treated group were 22.4 and 8.6 , respectively. It is worthy of note that before treatment, SPQ scores were similar for both groups $[\mathrm{t}(36)=1.42, \mathrm{p}>.1]$. SPQ scores were comparable to the mean scores that Fredrikson (1983) and de Jong and Merckelbach (1991) reported for their phobic groups. Subjects also underwent a behavioral approach test (BAT), both before and after treatment. The BAT was scored on a 13-point scale ranging from $0(300 \mathrm{~cm})$ to 12 (spider on hand). The treated subjects strongly benefited from therapy as indexed by SPQ scores $(\mathrm{t}(19)=-11.1, \mathrm{p}<.001$; $\underline{M} s=21.6$ and 9.0$)$, and by BAT scores $(\mathrm{t}(19)=14.4, \mathrm{p}<.001 ; \underline{\mathrm{M}} \mathrm{s}=2.5$ and 9.1).

\section{Apparatus and Stimulus Materials.}

Three categories of stimuli were used: four different slides depicting flowers, four different slides depicting spiders, and four different slides depicting weapons (knives and guns directed toward the subject; e.g., Hughdahl \& Johnson, 1989). The slides were projected onto a white screen $(80 \mathrm{~cm} \times 120$ $\mathrm{cm}$ ), approx. $3 \mathrm{~m}$ in front of the subject. A Kodak Carousel was used for stimulus presentation. Three types of outcomes occurred during the experiment: a 1-s shock, a 1-s tone, or nothing at all. Electric shocks (dc) were delivered from a specially designed shock generator $(0-40 \mathrm{~mA})$ and administered to the subjects' lateral side of t'ie upper right arm through two electrodes ( $8 \mathrm{~mm}$ diameter $\mathrm{Ag}-\mathrm{AgCl})$. Tones were delivered by a tone generator $(60 \mathrm{~Hz}$, $50 \mathrm{~dB}$ ) connected to a loudspeaker inside the experimental (sound attenuated) 
room. Electrodermal activity was recorded from two Beckman $\mathrm{Ag}-\mathrm{AgCl}$ electrodes, placed on the medial phalanges of the second and third finger of the non-dominant hand. The electrodes were filled with an isotonic paste and conected to a Beckman Skin Conductance Coupler (type 9844). Stimulus presentation, delivery of tones and shocks, and intertrial intervals were controlled by a PDP Minc-II laboratory computer.

\section{Procedure}

Subjects were randomly assigned to one of two groups. Subjects in the aftertreatment group first underwent a 2.5 -hr (approximately) one-session in vivo exposure (as described by Öst, 1989) before they participated in the experiment. Subjects in the before-treatment group participated in the experiment before they received exposure therapy. The one-session treatment employed consists of exposure in vivo and modeling and has been found to yield good immediate and longterm results (Merckelbach, de Jong \& Arntz, 1991; Öst, 1989).

During the experiment, subjects were seated in a comfortable chair in a soundattenuated room. A one-way screen separated the experimental and the registration room. The subject was told that it was her task to determine the relationship between categories of slides and outcomes. Then electrodes were attached. Next, shock intensity level was determined using a shock work-up procedure. Stepwise, electrical current was increased until the subject indicated that the shock was uncomfortable but not painful. After the subject confirmed that the task was clear to her, she was left alone and the lights were dimmed. Subjects were exposed to 72 slides each of 8 -s duration. Three different categories were used: flowers (neutral stimuli), weapons (ontogenetically fear-relevant stimuli), and spiders (phylogenetically fear-relevant stimuli). One of three possible outcomes occurred at slide disappearance: a 1-s shock (aversive outcome) or a 1-s tone or nothing at all (neutral outcomes). Across all trials, the conditional probability of any outcome given the prior occurrence of any category of slide was $33 \%$ and so was the probability of occurrence of each category of slide and each type of outcome. Intertrial intervals ranged from 10 to $30 \mathrm{~s}$. Stimulus-outcome combinations were randomly distributed across trials, with the restriction that on two successive trials no identical stimulus-outcome combinations occurred. To cancel out order effects, three different sequences were used.

At the end of the experiment, subjects completed the Covariation Questionnaire (CQ). The CQ comprised two sections. Questions in the first section asked subjects to estimate the percentage of occurrence of each outcome given the prior occurrence of each slide type. In addition, subjects were asked to indicate their confidence in each reported probabilty estimate. For all estimates in this section, $100 \mathrm{~mm}$ visual analogue scales (VASs) were used ranging from $0 \%$ to $100 \%$. The second section consisted of questions that asked subjects to indicate which type of slides was most often followed by 
a given outcome (forced choice). Again, subjects were asked to indicate on a VAS how certain they were of their choice, with $0 \%$ meaning not certain at all and $100 \%$ meaning very certain.

\section{Data Reduction and Analysis}

$C Q$. Contingency estimates were subjected to a set of a priori t-tests in order to evaluate (a) whether the spider/shock contingency estimates differed from the weapon/shock, flower/shock, spider/tone, and spider/nothing contingency estimates (b) whether the posttreatment estimates of the spider/shock contingency differed from the pretreatment spider/shock contingency estimates and (c) whether the difference between the reported $\mathrm{P}$ (spider/shock) and $\mathrm{P}$ (weapon/shock) changed as a result of treatment, i.e., whether there is an interaction between stimulus (spider/weapon) and treatment (before/after). The reported confidence in the spider/shock contingency estimates before and after treatment were subjected to between-groups a priori $t$ tests.

For both the pre-treatment and the post-treatment group, forced choice data were subjected to 2 Chi Square tests. The following contrasts were tested: a. The number of subjects reporting the spider slide as being the best predictor for shock outcome vs. the numbe: of subjects reporting the flower or weapon slides as being the best predictor of shock outcome $(H=0: 1 / 3$, $2 / 3$ ); $b$. The number of subjects indicating the spider slides vs. the number of subjects indicating the weapon slides as being the best predictors of shock $(\mathrm{H}=0: 1 / 2,1 / 2)$.

In order to control for experimentwise error, a Dunn-Bonferroni strategy was adopted. Therefore, each of the four within group comparisons of the contingency estimates was tested at an alpha of $.05 / 4=.0125$ [see Tomarken et al. (1989), for a detailed justification of this strategy]. For the within group comparisons of the forced choice data alpha was set at $.05 / 2=.025$.

To explore the relationship between covariation bias and treatment outcome, a Pearson's correlation was computed between pre-treatment spider/shock contingency estimates and post-treatment SPQ scores.

Skin conductance. Two different skin conductance responses were scored: The first interval response (FIR; $1-4 \mathrm{~s}$ after CS onset, for each trial) and the unconditioned response (UCR; on shocked trials). the FIR is said to reflect an orienting response (e.g., Stern \& Walrath, 1977). The UCR was considered as an index of shock impact.

Electrodermal data were subjected to a priori $t$-tests to evaluate a. Whether FIRs on spider trials differ from FIRs on weapon or flower trials; b. Whether UCRs on shocked spider trials differ from UCRs on shocked weapon or flower trials; and c. Whether there is an interaction between the relative strength of electrodermal responding on spider trials and group (treated vs. untreated subjects). 


\section{Results}

As is evident from Figure 2.6, untreated spider phobics dramatically overestimated the spider/shock association. All planned comparisons reached significance: The spider/shock contingency estimates were significantly higher than both the weapon/shock $[\mathrm{t}(17)=2.71, \mathrm{p}<.01]$ and flower/shock $[\mathrm{t}(17)=3.97$, $\mathrm{p}<.01]$ contingency estimates. Additionally, the spider/shock contingency estimates were significantly higher than the spider/tone $[\mathrm{t}(17)=3.85, \mathrm{p}<.01]$ and the spider/nothing, [t(17) $=3.00, \mathrm{p}<.01]$ contingency estimates. In contrast to untreated subjects, treated subjects did not show a covariation bias. None of the planned comparisons reached significance $[\operatorname{ts}(19)<1.25$, ps $>$.20].

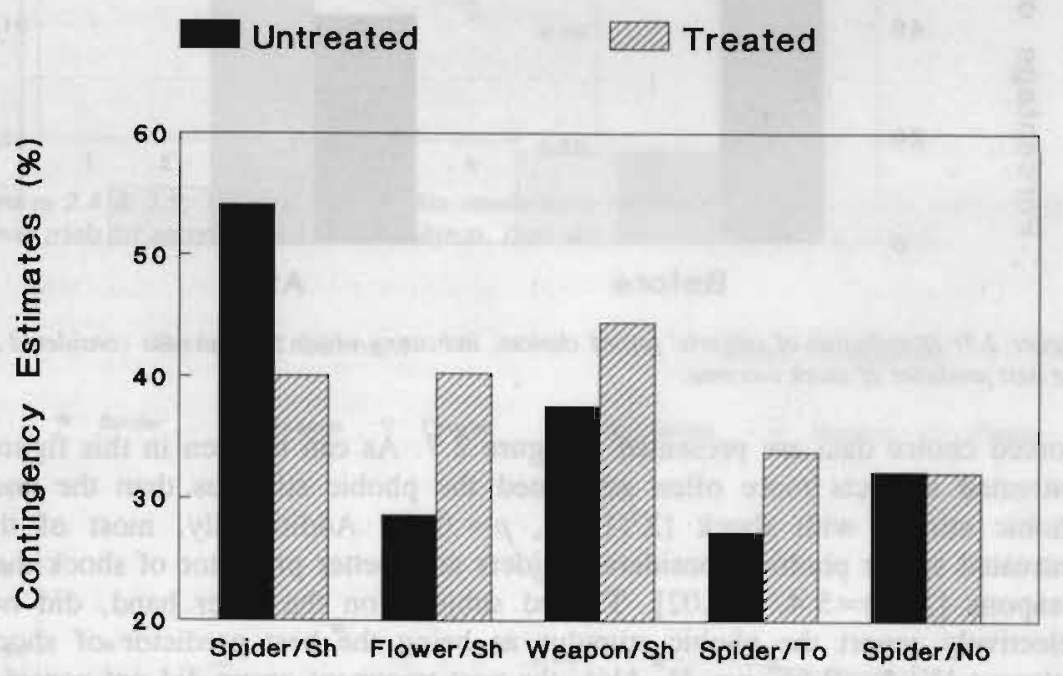

Figure 2.6: Most relevam a posteriori reported contingency estimates of the treated and untreated spider phobics (sh=shock; to=tone; no=no outcome).

A between-groups $\underline{t}$ test indicated that the spider/shock association was stronger in untreated than in treated phobics $[\mathrm{t}(36)=2.20, \mathrm{p}<.05]$. Moreover, the confidence of the spider/shock contingency estimates was higher in untreated subjects than in treated subjects. However, this effect only reached borderline significance [t(36) $=1.88, \mathrm{p}=.07]$ ( $\mathrm{M} s$ of confidence percentages $=$ 59 and 41 , respectively). Overall, contingency estimates and reported confidence correlated only weakly, Pearson's $\mathrm{I}(38)=.17, \mathrm{p}=.02$. The spider/shock contingency estimates of untreated subjects were not related to post-treatment SPQ scores (therapy success), Pearson's $\mathrm{I}(18)=-.12, \mathrm{p}=.31$.

The difference in contingency estimates between spider and weapon 
slides was significantly smaller in treated as compared to untreated subjects $[t(36)=2.45, p=.02]$, means being -4.4 and 16.6 , respectively. This finding suggests that, as a result of treatment, subjects' weapon/shock contingency estimates increased relatively, whereas the spider/shock contingency estimates decreased relatively.

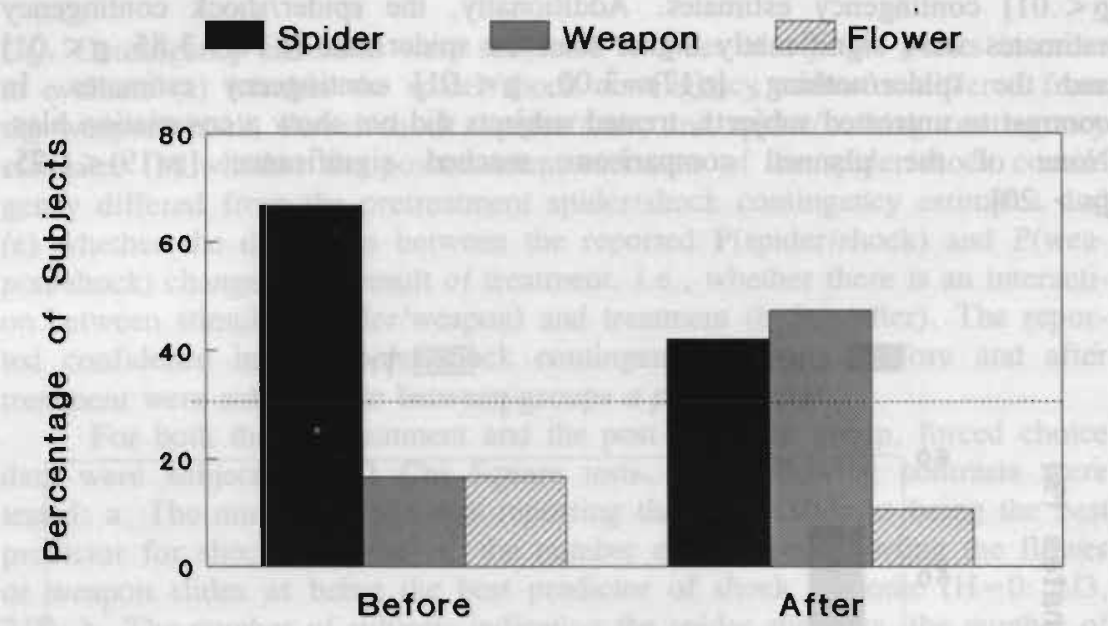

Figure 2.7: Distribution of subjects' forced choices, indicating which stimulus was considered as the best predictor of shock outcome.

Forced choice data are presented in figure 2.7. As can be seen in this figure, untreated subjects more often associated the phobic stimulus than the nonphobic stimuli with shock $\left[\mathrm{X}^{2}(1)=9, p=.003\right]$. Additionally, most of the untreated spider phobics considered spiders as a better predictor of shock than weapons $\left[\mathrm{X}^{2}(1)=5.4, p=.02\right]$. Treated subjects on the other hand, did not selectively report the phobic stimulus as being the best predictor of shock outcome $\left[\mathrm{X}^{2}(1)=0.66, p=.4\right]$. Also, the post treatment group did not consider spiders as a better predictor of shock than weapons $\left[\mathrm{X}^{2}(1)=0.06, p=.8\right]$. Moreover, treated subjects who reported that spiders were the best predictors of shock were significantly less certain about their choice than untreated subjects making the same choice $[t(18)=2.42, p=.03]$, means being 31.3 and 56.8 , respectively.

FIR. Figures 2.8 and 2.9 show the FIRs. Within-subjects t-tests indicated both treated and untreated subjects responded with larger FIRs to phobia-relevant slides than to phobia-irrelevant slides $[t(19)=2.67, p=.015]$ and $[t(17)=3.53$, $p=.003]$, respectively. There was no interaction between group (treated vs. untreated) and stimulus type (phobic vs. non-phobic) $[\mathrm{t}(36)=0.24]$, indicating that the relative strength of FIRs on spider slides did not differentiate between treated and untreated subjects. 
Before Treatment

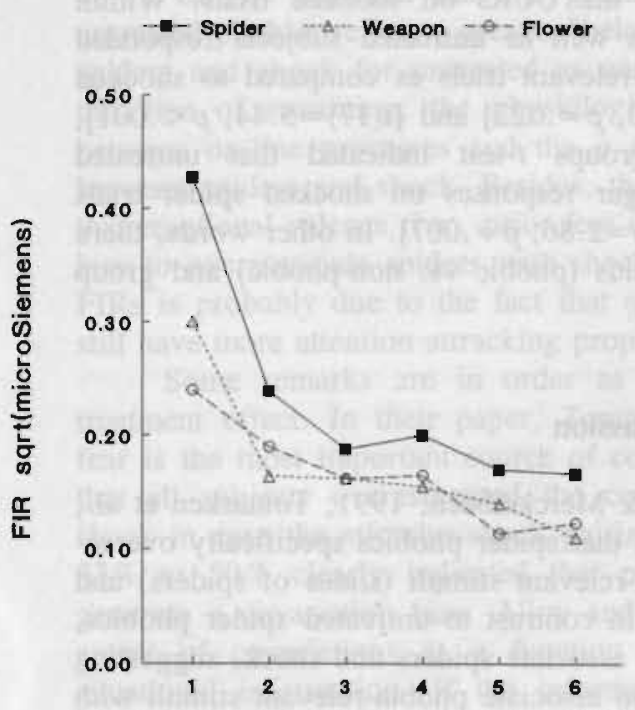

After Treatment

Figures 2.8 \& 2.9: The first interval skin conductance responses (FIRs) on spider, weapon, and flower trials for untreated and treated subjects. Data are presented in blocks of 4 trials.

\section{Before Treatment}

- Spider

A. Weapon -e-Flower

อ
1.00

总

\subsection{0}
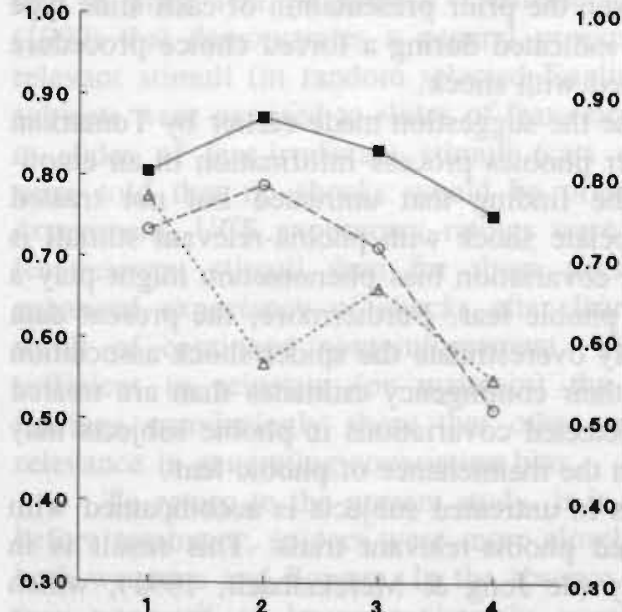

0.30
2

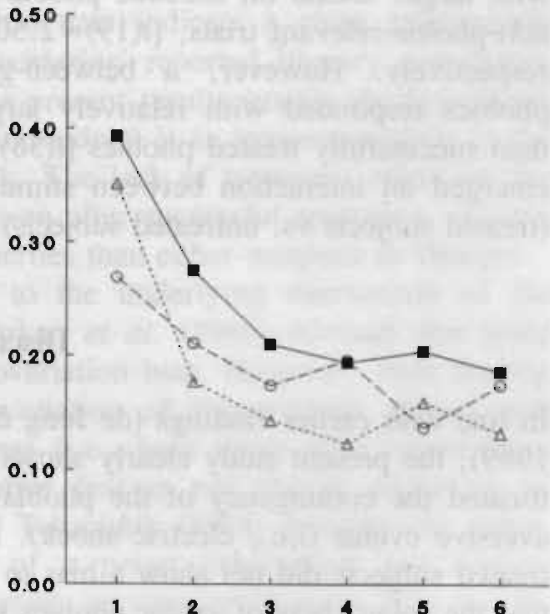

- Spider

Weapon - $\theta$-Flower 
UCR. Figures 2.10 and 2.11 depict the UCRs on shocked trials. Within subjects $t$-tests showed that treated as well as untreated subjects responded with larger UCRs on shocked phobia-relevant trials as compared to shocked non-phobia-relevant trials, $[t(19)=2.50, p=.022]$ and $[t(17)=5.44, p<.001]$, respectively. However, a between-groups $t$-test indicated that untreated phobics responded with relatively larger responses on shocked spider trials than successfully treated phobics $[t(36)=2.86, p=.007]$. In other words, there emerged an interaction between stimulus (phobic vs. non-phobic) and group (treated subjects vs. untreated subjects).

\section{Discussion}

In line with earlier findings (de Jong \& Merckelbach, 1991; Tomarken et al., 1989), the present study clearly shows that spider phobics specifically overestimated the contingency of the phobia-relevant stimuli (slides of spiders) and aversive events (i.e., electric shock). In contrast to untreated spider phobics, treated subjects did not show a bias to associate spiders and shock, suggesting that the robust bias of spider phobics to associate phobia-relevant stimuli with aversive events can be modified by behavioral treatment. In addition, it was found that treated subjects were less certain about the covariation of stimuli and outcomes than untreated subjects. Moreover, the present data seem to imply that as a result of treatment, the bias of associating spiders and shock relatively diminishes, while the estimates concerning the contingency between alternative danger-related stimuli (i.e., weapons) and shock increase relatively. This pattern of results is evident both when subjects estimated the percentage of occurrence of shock outcome given the prior presentation of each slide type $[\mathrm{P}($ shock/slide $)]$ and when subjects indicated during a forced choice procedure which slide was most often associated with shock.

The present findings underline the suggestion made earlier by Tomarken et al. (1989), that (untreated) spider phobics process information in an emotion-congruent way. In addition, the finding that untreated but not treated phobics show a strong bias to associate shock with phobia-relevant stimuli is in line with the hypothesis that the covariation bias phenomenon might play a critical role in the maintenance of phobic fear. Furthermore, the present data show that untreated phobics not only overestimate the spider/shock association but are also more confident about their contingency estimates than are treated subjects. This high confidence in detected covariations in phobic subjects may add to the robust covariation bias in the maintenance of phobic fear.

The revealed covariation bias in untreated subjects is accompanied with relatively greater UCRs on shocked phobia-relevant trials. This result is in agreement with our previous study (de Jong \& Merckelbach, 1991), which also showed that covariation bias was mimicked by differentially heightened electrodermal responding. The greater UCRs on phobia-relevant trials can be attributed to a match between the primary affective response organization of both stimuli (i.e., spider and shock) (e.g., Lang, 1985). From this perspecti- 
ve, it can be reasoned that the greater UCRs on shocked spider trials in untreated phobics reflect a greater "belongingness" of the emotional valence of spiders and shock for untreated as compared to treated subjects. Following this line of reasoning, the physiological data indicate a close relationship between on-line processes and the a posteriori reported illusory correlation between spiders and shock. Besides, the present results sustain the hypothesis that emotional valence (i.e., prior fear of spiders) is an important factor in the bias to overassociate spiders with shock. The lack of treatment effect on the FIRs is probably due to the fact that even after successful treatment, spiders still have more attention attracking properties than either weapons or flowers.

Some remarks are in order as to the underlying mechanism of the treatment effect. In their paper, Tomarken et al. (1989) stressed that prior fear is the most important source of covariation bias. However, their finding that all subjects overestimated the covariation of fear-relevant stimuli and shock in case the stimulus-shock pairings (i.e., base rates) were raised from $33 \%$ to $50 \%$ clearly indicated that prior fear is not always necassary to generate a covariation bias. Alloy and Tabachnik (1984) describe the assessment of covariations as a function of $a$ priori expectation and current situational information. If the information is very ambiguous (like in the present paradigm), subjects predominantly rely on preexperimental expectancies. It seems obvious that prior fear is one source of such preexperimental expectancies (e.g., "shocks will be presented after spider slides"). However, alternative sources should be considered. Cultural information, for instance, might be an important additional source of preexperimental expectancy bias in the present context. In other words, because of the cultural connotation in the Netherlands, subjects may expect spiders to have aversive outcomes, irrespective of prior fear of spiders. Germane to this issue, is a study by Davey (1992) that demonstrates a general preexperimental expectancy bias to fearrelevant stimuli (in random selected English students). In that study (exp. 1) subjects were exposed to slides of fear-relevant stimuli (spiders and snakes) or to slides of fear-irrelevant stimuli (cats and pigeons). Even when subjects were told that no shocks would be presented in a particular stage of the experiment, UCS expectancy ratings were higher for subjects presented with fear-relevant stimuli than for those presented with pigeons or cats. The enhanced expectancy of shocks after fear-relevant stimuli disappeared as a result of continued nonreinforcement. Yet, a single CS-UCS pairing was sufficient to reinstate (or maintain) the expectancy bias (exp. 4). These findings convincingly show that other sources than prior fear can be of relevance in generating covariation bias.

To return to the present study, it is likely that, as a result of prior fear before treatment, spiders were more closely associated with danger than were both weapons and flowers. In the absence of prior fear, spiders and weapons may expected to be approximately equally related to danger, because of cultural connotations. Consequently, the presence of two competing threatrelated stimuli is likely to undermine the covariation bias between spiders and shock after treatment. The absence of a bias of associating shock with phobia- 
relevant stimuli after treatment and the shift from spiders towards weapons as the most frequently reported predictor of shock outcome in the present study, are in line with this hypothesis. The lack of treatment effect on covariation bias as was reported by de Jong and Merckelbach (1991) can also be interpreted along these lines (note, that in that study no additional danger-related stimuli were employed). That is, it may wel be that a priori differential UCS expectancy of treated subjects and low-fear controls in that study (for instance generated by residual fear or cultural connotation) was sufficient to induce a considerable bias to associate spiders with shock in the absence of alternative danger-related stimuli.

In the present study, no relationship was found between pre-treatment spider/shock contingency estimates and treatment outcome (as indexed by post-treatment SPQ scores). Thus, although a strong bias of associating phobic stimuli with aversive events may act in a way to confirm fear, it does not seem to have an impact on (short-term) therapy prognosis. However, it should be noted that in the present study, the length of treatment varied among subjects (range 2 to 3 hours). It can not be ruled out, therefore, that strength of bias does covary with length of treatment (and long-term treatment outcome).

To summarize, the current data show 1 that spider phobics dramatically overestimate the contingency between phobic stimuli and aversive events; 2 that untreated spider phobics have more confidence in their (distorted) covariation detection than treated subjects; 3 that covariation bias can be reduced by behavioural therapy; 4 that covariation bias is accompanied by differentially heightened electrodermal responding; and 5 that the strength of the covariation bias seems not to be related to therapy outcome. 
3.a PATHWAYS TO COVARIATION BIAS: SUGGESTIVE COINCIDENCES

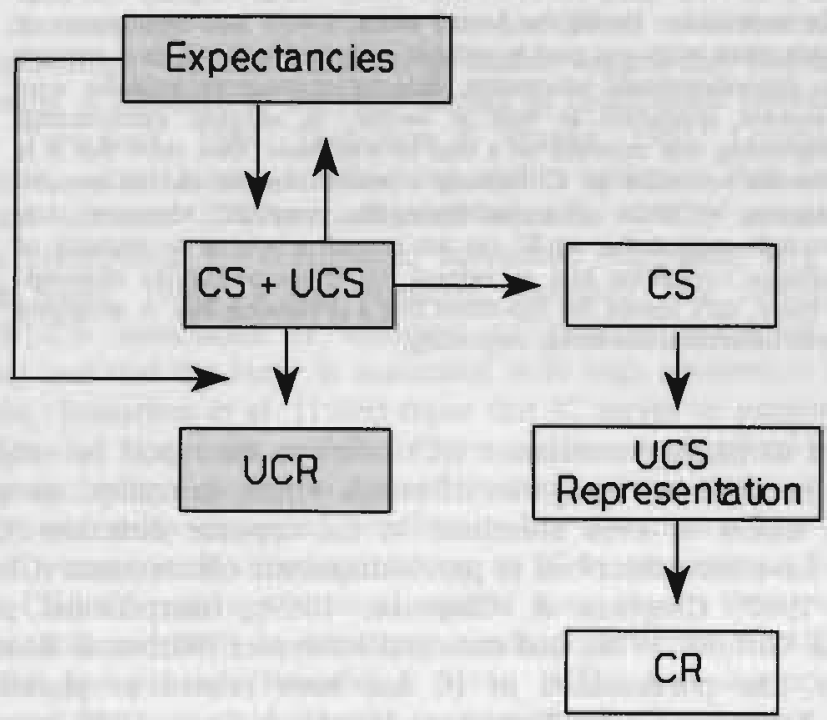

This chapter is based on: De Jong, P.J., Merckelbach, M., \& Arntz, A. (1990). Illusory correlations, on-line probability estimates, and electrodermal responding in a (quasi) conditioning paradigm. Biological Psychology, 31, 201-212.

Acknowledgements: The authors are grateful to Professor M.A. van den Hout for his thoughtful comments on an earlier version of this paper. Furthermore we would like to thank Peter Muris for his assistance during the data acquisition. 


\title{
Illusory Correlation, On-line Probability Estimates, and Electrodermal Responding in a (quasi) Conditioning Paradigm
}

\begin{abstract}
Twenty normal subjects were exposed to two series of two different slides randomly paired with the occurrence or non-occurrence of shock. The first series started with a greater number of pairings of one slide with shock ("target slide") than the other to induce an illusory correlation (IC). Meanwhile, across all trials shock/slide contingency was equal for both slides. During the second series, a truly random sequence of slide-shock trials was used in order to examine whether IC was resistant to disconfirmational information. Subjects' contingency estimates were recorded aposteriori as well as on-line. In addition, electrodermal responding was recorded on a trial by trial basis. Data show that it is possible to induce an IC between a neutral stimulus and an aversive outcome by means of a conditioning-like procedure. Moreover, data strongly suggest that an IC can act in such a way as to maintain or enhance covariation bias as indexed by on-line probability estimates. Finally, data sustain the hypothesis that a covariation bias is associated with differential autonomic responding.
\end{abstract}

The concept of illusory correlation (IC) refers to the report by subjects of a covariation between two categories of events which, in reality, are correlated to a lesser extent or even correlated in the opposite direction (Chapman, 1967). ICs have been described in psychodiagnostic observations (Chapman \& Chapman, 1967; Chapman \& Chapman, 1969), interpersonal perception (Hamilton \& Gifford, 1976), and statistical inferences (Nisbett \& Ross, 1980).

Recently, the phenomenon of IC has been related to phobic anxiety (Mineka \& Tomarken, 1989; Tomarken, Mineka \& Cook, 1989; experiment 1 to 3). In the studies of Tomarken et al. (1989), phobic and control subjects were confronted with a sequence of 72 slides. Three different categories (one phobic and two neutral) were used. Slide offset was followed by one of three possible outcomes: A tone, an aversive shock, or nothing at all. The base-rate probabilities of all slides and outcomes were equal and so were the conditional probabilities (i.e., $33 \%$ ). Consequently, stimuli and outcomes were equally correlated. At the end of the experiment, subjects were asked to estimate the covariation between all stimulus/outcome combinations. In contrast to low-fear subjects, phobic subjects dramatically overestimated the covariation between phobic stimuli (i.e., slides of snakes or spiders) and shock outcome. Thus, phobic subjects reported an illusory correlation between phobic stimuli and aversive outcomes. The authors suggested that the IC phenomenon might be an important factor in the etiology and maintenance of phobic anxiety. Acknowledging the potential strength of Tomarken et al.'s laboratory model, the present study sought to further elaborate the model.

First, a question not empirically addressed by Tomarken et al. (1989) concerns the origin of the observed IC. The authors suggest that the IC reflects 
biased processing of information during the experiment. An alternative explanation might be that phobic $S$ s entered the laboratory with a habitual tendency to overassociate phobic cues with negative outcomes. The IC may have reflected an enduring phobic memory network and not necessarily biased on-line processing. A first aim was to see whether a paradigm comparable to that of Tomarken et al. (1989) allows for the detection of on-line covariation bias. More specifically, we examined whether an aposteriori covariation bias (IC) would occur after presenting a "suggestive" sequence of slide/shock trials (see below) to non-phobic Ss and, if so, whether this aposteriori IC is related to on-line probability ratings. Second, the model may gain in strength if it could be demonstrated that covariation bias has effects on autonomic "conditioned" responding. Note that a number of studies suggest that human covariation judgement is sensitive to the same factors as conditioned responding (e.g., Alloy \& Tabachnik, 1984; Dickinson \& Shanks, 1985; Lovibond, 1988). Furthermore, recent human conditioning studies underline that awareness of conditioned stimulus (CS)/unconditioned stimulus (UCS) covariation is a necessary prerequisite for conditioned autonomic responding (e.g., Dawson, Schell, \& Banis, 1986). It was predicted, therefore, that illusory belief in close CS/UCS association is sufficient to produce heigtened autonomic responding and that the latter is associated with high aposteriori covariation bias. Third, Tomarken et al. (1989) argue that IC serves to maintain phobias; this implies that ICs, once established, are resistant to "extinction", i.e., do not disappear during exposure to disconfirmational information.

The present study examined whether on-line covariation bias is, indeed, resistant to extinction. It aimed at inducing an IC by presenting a "suggestive" series of CS/UCS pairings. Animal studies have shown that truly random control procedures result in excitatory conditioning when CS-UCS pairings occur before non-pairings (Benedict \& Ayres, 1972). A similar primacy-effect was observed in social-psychological experiments: Early successes in a purely chance task (e.g., coin tossing) leads to overestimation of past success and increased expectation of future success (Langer \& Roth, 1975). Adopting an analogous strategy, the present study attempted to induce an IC between a neutral stimulus and an aversive outcome (shock). Subjects were exposed to two series of slides randomly paired with the occurrence or non-occurrence of shock. For both series, base-rate probabilities of the two slides were equal as were the conditional probabilities (50\%). The first series (IC induction phase) started, however, with a number of pairings of one slide category (the "target" slide) with shock. Across all trials, shock/slide contingency was equal for both slides. During the second phase, subjects were exposed to a random series of slide/shock pairings (IC extinction phase). Again shock/slide contingency was equal for both slides. The extinction phase was used in order to examine whether IC was resistant to disconfirmational information (no "suggestive" order of slide-shock trials). On-line probability estimates and electrodermal responding were recorded concurrently on a trial by trial basis. At the end of the experiment, subjects were asked to make judgements concerning the degree of covariation between slides and outcomes. 
It was predicted that subjects would report an (aposteriori) IC between the "target" slide and the aversive outcome. Furthermore, we examined whether subjects reporting an aposteriori IC react with conditioning-like SCR components and biased on-line probability estimates to the target slide as compared to the control slide.

\section{Method}

\section{Subjects}

Twenty healthy undergraduate volunteers ( 12 females) were paid for participating in this experiment. Mean age was 21 yrs (range 18-25 yrs).

\section{Stimulus Materials and Apparatus}

Two stimuli were used throughout the experiment: A slide depicting an "X" and a slide depicting an "O". Slides were projected by a Kodak Carousel onto a screen $(80 \times 120 \mathrm{~cm})$, approx. $2 \mathrm{~m}$ in front of the subject.

Shocks were delivered from a Siemens Neuroton 623 apparatus and administered to the subject's lateral side of the lower (right) leg just above the ankle through two electrodes $(8 \mathrm{~mm}$ diameter $\mathrm{Ag}-\mathrm{AgCl})$. During the experiment, electrodermal activity was recorded from two Beckman $\mathrm{Ag}-\mathrm{AgCl}$ electrodes ( $8 \mathrm{~mm}$ diameter), placed on the medial phalanges of the second and third finger of the non-dominant hand. The electrodes were filled with an isotonic paste and connected to a Beckman Skin Conductance Coupler (type 9844). Frequency and depth of ventilation was recorded by means of a Beckman respiratory belt connected to a Beckman Voltage/Pressure Coupler.

For measuring on-line probability estimates (PE), a rotary lever mounted on a scale was used (range 180 degrees) positioned in front of the seated subject (e.g., Furedy \& Schiffmann, 1973). Subjects were informed that the scale represented a continuum. The horizontal left $(0)$, vertical, and horizontal right (100) positions represented absolute certainty that the shock would not occur, complete uncertainty, and absolute certainty that the shock would occur, respectively. The output from the "PE-device" was recorded on a Beckman Polygraph. Stimulus presentation, shocks and intertrial intervals were controlled by a PDP Minc-II computer.

\section{Procedure}

After introduction to the laboratory, electrodes and respiratory belt were attached to the subject. Following this, a shock work-up procedure was carried out in order to set the intensity level of the shock. Shock level was increased in steps of $0.2 \mathrm{~mA}$ until the subject indicated that the intensity was 
uncomfortable but not painful. After the completion of this procedure, the subject was informed about the experimental task. It was explained that (s)he had to determine whether or not there was a relationship between a particular slide and a particular outcome following the slide. Furthermore, subjects were instructed to use the "PE-device" during each slide presentation. PE output was used as an index of subject's on-line beliefs about the likelihood of shock occurrence (in some studies referred to as "subjective contingency"; e.g., Schiffmann \& Furedy, 1977).

The experiment consisted of 52 trials: $26 \mathrm{X}$-slides and 26 O-slides. Both slide categories were followed half of the time ( 13 trials) by a 2 -s shock. Slide duration was always $8 \mathrm{~s}$. The intertrial interval varied from 10 to $30 \mathrm{~s}$ with a mean of $20 \mathrm{~s}$. The 52 trials were grouped into two phases. The first ("induction") phase (32 trials) was analogous to the "primacy-series" used by Langer and Roth (1975). This phase aimed at inducing an illusory correlation between the target slide and shock. In half of the subjects the $\mathrm{X}$ was used as target slide, in the other half the $\mathrm{O}$. For the sake of convenience, the target and the control stimulus will hereafter be referred to as the $T$ and $C$ slide, respectively. The first phase began with a random series of $3 \mathrm{C}$ slides of which 1 was followed by shock and $7 \mathrm{~T}$ slides of which 5 were followed by shock. During the remaining 22 trials, slide-shock contingencies were such that the positive T-shock correlation was down-scaled to a momentary deviation in what might be a perfectly random sequence of slides and shocks.

The second ("extinction") phase consisted of 20 trials. During this phase, slides and shocks were presented randomly, with the restriction that a given slide was never followed by a shock on two or more consecutive trials. The interval between the first and the second phase was $30 \mathrm{sec}$. It should be stressed that for both phases base-rate probabilities of the $\mathrm{C}$ and $\mathrm{T}$ slides were equal and so were the conditional probabilities $(50 \%)$.

At the end of the experiment, subjects completed the Probability Questionaire (PQ) on which they indicated their perceptions of the relationships between slides and outcomes. The PQ comprised two sections. Questions in the first section asked subjects to estimate the percentage of times each of the two outcomes (shock/no shock) occurred given the prior presentation of each of the two slides $(\mathrm{T} / \mathrm{C})$. The second section asked subjects to estimate the base-rates of each slide type and each outcome during the 52 trials. For all estimates visual analogue scales (VAS) were used ranging from 0 to $100 \%$.

\section{Data Analysis and Reduction}

Skin conductance response. For each trial, three different SCR components were scored: the first interval response (FIR; $1-4 \mathrm{~s}$ after CS onset), the second interval response (SIR; 4-9 $\mathrm{s}$ after CS onset), and the third interval omission response (TOR; $1-4 \mathrm{~s}$ after CS offset, on non-reinforced trials). The FIR component is said to reflect an orienting response (e.g., Stern \& Walrath, 1977), the SIR component is regarded as an anticipatory response due to the 
preparation for the occurrence of the UCS (e.g., Grings, 1969), while the TOR component is thought to reflect "suprise" about the non-occurrence of shock (e.g., Seligman, Maier, \& Solomon, 1971; see, however, Maltzman, 1987, for a different view). A conditioned like SCR was assumed to have occurred when FIR, SIR, and TOR components occurred more frequently and the UCR component decreased more during the $T$ slide trials than during the C slide trials. Following the criteria of Stern, Ray and Davis (1980), responses associated with ventilatory irregularities were omitted. SCR data were analyzed as magnitudes and probabilities. Because preliminary inspection of the FIR, SIR, and TOR magnitude data showed a large number of zero responses, further analyses were performed on frequency data (Stern, Ray \& Davis, 1980). The electrodermal data were subjected to a 2 (slide type) $\times 2$ (phase) ANOVA with both factors being a within-subjects factor.

On-line probability estimates. For both slide types and for each phase of the experiment, the mean PE was computed. The mean scores were used as dependent measures. To test differences in PE between the two slides during the two parts of the experiment, a 2 (slide type) $X 2$ (phase) ANOVA was conducted with both factors being a within-subjects factor.

Probability Questionnaire. We tested whether the $\mathrm{P}($ shock/T) and or $\mathrm{P}($ shock/C) estimates differed from $50 \%$. It was predicted that subjects would overestimate the covariation between T-slide and shock but not between Cslide and shock.

As the present study was primarily concerned with concommitants of the "illusory correlation" phenomenon, only subjects reporting an IC (P(shock/T) $>$.5) were included in the final analyses of $\operatorname{SCR}(n=16)$. For all subjects, Pearson's product-moment correlations were computed between PE and SCR data on a trial by trial basis. After Fischer's $\mathrm{Z}$ transformation these correlations were averaged. Finally, in order to explore the interrelationship between the aposteriori reported IC and the on-line reported probability estimates a multiple regression analysis (backward) was carried out. The aposteriori reported $\mathrm{P}($ shock/T) was used as the dependent variable and mean $\mathrm{PE}($ shock/T) "induction" phase, mean PE(shock/C) "induction" phase, mean $\mathrm{PE}($ shock/T) "extinction" phase, and mean $\mathrm{PE}($ shock/C) "extinction" phase were used as predictor variables.

\section{Results}

\section{Covariation Estimates (aposteriori)}

Figure 3.1 shows PQ data. Overall, subjects overestimated the probability of shock given a $T$-slide $($ mean $=59.6 ;$ s.d. $=13.9)$ compared to their estimated probability of shock given a C-slide (mean $=46.2$ s.d. $=14.8$ ). Paired $\mathrm{t}$-tests showed that the reported $\mathrm{P}($ shock/T) differed significantly from $50 \%$ 
$[\mathrm{t}(19)=3.05 \mathrm{p}<0.05]$ whereas the reported $\mathrm{P}($ shock $/ \mathrm{C})$ did not $[\mathrm{t}(19)=-1.16$, $\mathrm{D}=0.26]$ Base-rate estimates did not differ significantly.

Ema Control

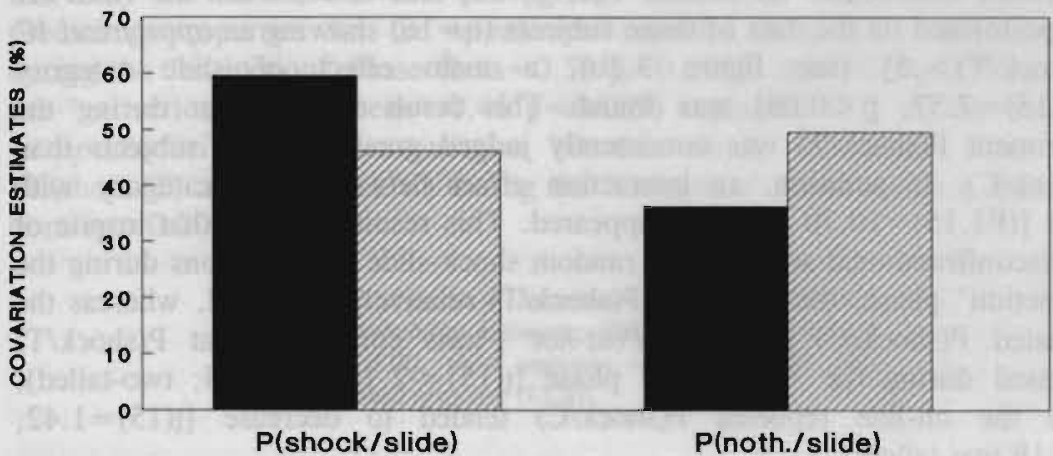

Figure 3.1: Subjects' aposteriori covariation estimates.
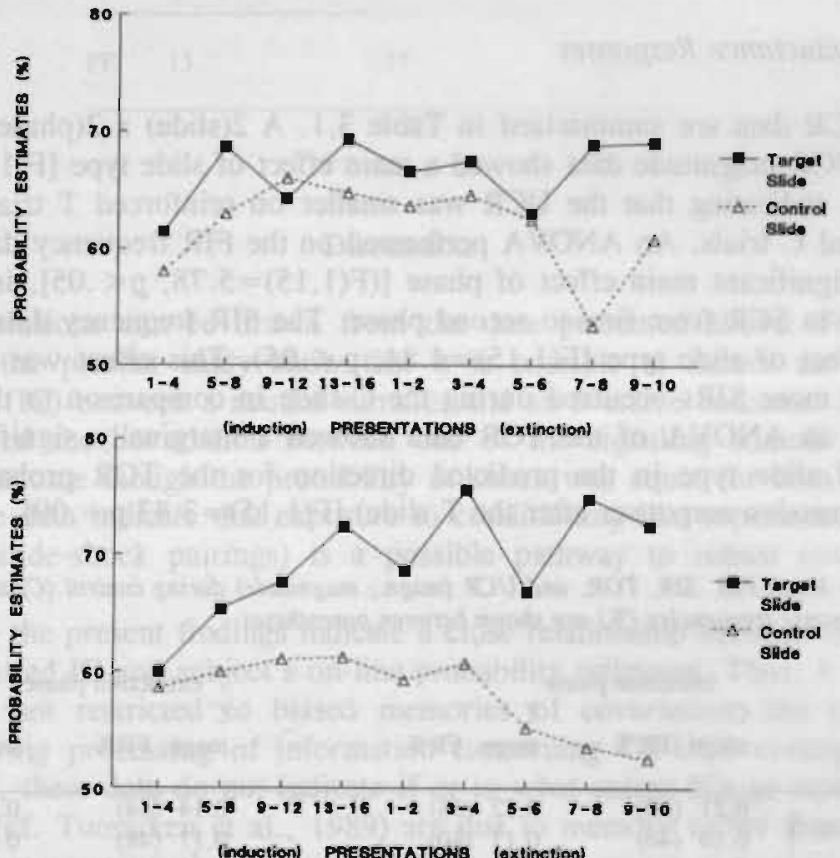

Figure 3.2a: Mean on-line estimates of probability of shock (PE) during both phases $(n=20)$. $P E$ during the "induction" phase is depicted in blocks of 4 presentations; PE during the "extinction" phase is depicted in blocks of 2 presentations (b) Mean on-line estimates of probability of shock $(P E)$ during both phases for those subjects reporing an aposteriori IC $(n=16)$. PE during the "induction" phase is depicted in blocks of 4 presentations; PE during the "cxtinction" phase is depicted in blocks of 2 presentations. 
Figure 3.2a reflects the mean on-line probability estimates. A 2 (T vs C) $\times 2$ (induction vs extinction) ANOVA performed on the PE data yielded no significant effects [for all effects: $F(1,19)<1$ ]. However, when the ANOVA was performed on the data of those subjects $(\mathrm{n}=16)$ showing an aposteriori IC $[\mathrm{P}($ shock $/ \mathrm{T})>.5]$ (see figure $3.2 \mathrm{~b}$ ), a main effect of slide category $[(\mathrm{F} 1,15)=7.57, \mathrm{p}<0.05]$ was found. This result implies that during the experiment $\mathrm{P}($ shock $/ \mathrm{T})$ was consistently judged greater by the subjects than $\mathrm{P}($ shock/C). In addition, an interaction effect between slide category with phase $[(F 1,15)=10.70 \mathrm{p}<0.05]$ appeared. This result suggests that inspite of the disconfirmational sequence of random shock-slide combinations during the "extinction" phase, the estimated $\mathrm{P}($ shock $/ \mathrm{T})$ relatively increased, whereas the estimated $\mathrm{P}($ shock/C) declined. Post-hoc t-tests confirmed that $\mathrm{P}($ shock/T) increased during the "extinction" phase [t $(15)=-2.19, \mathrm{p}<0.05$; two-tailed), while the on-line reported $\mathrm{P}($ shock/C) tended to decrease $[\mathrm{t}(15)=1.42$, $\mathrm{p}=0.18$ two-tailed $]$.

\section{Skin Conductance Responses}

Mean SCR data are summarized in Table 3.1. A 2(slide) $\times 2$ (phase) ANOVA on the UCR magnitude data showed a main effect of slide type $[F(1,15)=5.72$ $\mathrm{p}<.05$ ], indicating that the UCR was smaller on reinforced $\mathrm{T}$ trials than on reinforced $\mathrm{C}$ trials. An ANOVA performed on the FIR frequency data yielded only a significant main effect of phase $[(\mathrm{F}(1,15)=5.78, \mathrm{p}<.05]$, indicating a decrease in SCR from first to second phase. The SIR frequency data showed a main effect of slide type $[\mathrm{F}(1,15)=4.84, \mathrm{p}<.05)$. This effect was due to the fact that more SIRs occurred during the C-slide in comparison to the T-slide. Finally, an ANOVA of the TOR data showed a marginally significant main effect of slide type in the predicted direction for the TOR probability data (more omission responses after the $\mathrm{T}$ slide) $[\mathrm{F}(1,15)=3.43 \mathrm{p}=.09]$.

Table 3.1: Mean FIR, SIR. TOR, and UCR (magn.; magnirude) during control (C) and target (T) trials. Response frequencies (\%) are shown between parentheses.

\begin{tabular}{|c|c|c|c|c|}
\hline & induction & & extinction & \\
\hline & $\begin{array}{l}\mathrm{C} \\
\text { magn. FR\% }\end{array}$ & $\begin{array}{l}\mathrm{T} \\
\text { magn. FR\% }\end{array}$ & $\begin{array}{l}\text { C } \\
\text { magn. FR\% }\end{array}$ & $\begin{array}{l}\mathrm{T} \\
\text { magn. FR\% }\end{array}$ \\
\hline$\overline{F I R}$ & 0.21 (60) & $0.22(63)$ & $0.14(44)$ & $0.15(46)$ \\
\hline SIR & 0.19 (46) & $0.17(40)$ & $0.17(48)$ & 0.16 (45) \\
\hline TOR & $0.27 \quad(72)$ & 0.28 (77) & $0.20(59)$ & $0.20(65)$ \\
\hline UCR & 0.62 & 0.58 & 0.55 & 0.50 \\
\hline
\end{tabular}

Note: $\mathrm{UCR}=$ unconditioned response; FIR = first-interval response; $S I R=$ second-intervail response; TOR $=$ third-interval omission response. 
Pearson product-moment correlations of PE with SCR data (based on all subjects) are depicted in table 3.2. All correlations were low and non-significant.

The multiple (backward) regression analysis (based on all subjects) showed that only the on-line PE data during the "extinction" phase had significant predicting properties for the aposteriori reported $\mathrm{P}($ shock/T). PE(shock/T) during the "extinction" phase (beta $=0.53 \mathrm{p}=0.0035$ ) and $\mathrm{PE}($ shock/C) during the "extinction" phase (beta $=-0.46 \mathrm{p}=0.009$ ) explained $61 \%$ of the variance in aposteriori reported covariation between shock and target slide. The $\mathrm{PE}($ shock/T) and $\mathrm{PE}($ shock/C) during the "induction" phase had n.s. contributions $(\mathrm{p}>0.20)$

Table 3.2: Pearson $r$ correlations of subjects' $(n=20)$ on-line probability estimates (PE) with first interval response (FIR), third omission response (TOR), and unconditioned response (UCR).

\begin{tabular}{llll}
\hline & FIR & TOR & UCR \\
\hline PE & .13 & -.17 & -.12 \\
\hline
\end{tabular}

\section{Discussion}

Three conclusions can be drawn from the data presented above. First, the results of the present study show that it is possible to induce an illusory correlation (IC) between a neutral stimulus and an aversive outcome. Subjects overestimated the covariation between one of the signaling stimuli and the aversive outcome though the probability of shock was equal for both stimuli. Thus, these data indicate that exposure to conditioning-like experiences (e.g., incidental slide-shock pairings) is a possible pathway to robust covariation bias.

Second, the present findings indicate a close relationship between the aposteriori reported IC and subject's on-line probability estimates. Thus, it appears that IC is not restricted to biased memories of covariation, but can also emerge during processing of information concerning CS-UCS contingencies. Admittedly, these data do not indicate if or to what extent ICs as reported by phobic Ss (cf. Tomarken et al., 1989) are due to memory rather than on-line information processing phenomena.

Third, the data demonstrate that an IC, once induced, can become "selfsupporting": The subjects' on-line probability estimates of shock given " $T$ " [P(shock/"T")] increased during the second ("extinction") phase, whereas the $\mathrm{P}($ shock/ "C") declined. Obviously, an acquired IC can act in such a way as to promote the assessment of selective associations. This finding together with 
the revealed close relationship between the on-line and aposteriori covariation estimates provides additional support for the suggestion that covariation bias may be a prominent factor in the etiology and maintenance of phobic anxiety (Tomarken et al., 1989).

Grosso modo, the electrodermal data parallel the subjective indices of covariation estimation. A number of studies have shown that signalled shock elicits a smaller UCR than unsignalled shock (e.g., Morrow, 1966), a phenomenon which has been termed "UCR diminution". Although there is discussion about the theoretical meaning of this phenomenon (see e.g., Lykken, Macindoe \& Tellegen, 1972 vs. Furedy, 1975), UCR diminution has been found in electrodermal (Kimmel, 1966; Merckelbach \& de Jong, 1988), cardiovascular (Lykken, Macindoe, \& Tellegen, 1972), and evoked potential (Roth, 1973) studies. Of particular interest to the present context is a study of Grings and Sukoneck (1971) who demonstrated that UCR magnitude decreases as (objective) probability of UCS increases. These authors also suggest that the ability to predict with confidence the occurrence of shock (within a given physical probability category) is inversely related to the magnitude of response elicited by the shock. The present data fit nicely with the results of Grings and Sukoneck (1971). After successful IC induction, the pertinent slide had greater "predictive validity" (Lovibond, 1988) for shock occurrence than the control slide. In line with this, shocks preceded by the pertinent slide elicited smaller UCRs than shocks preceded by the control slide.

The TOR component is thought to reflect the arousal of an orienting response precipitated by breaking the expected CS-UCS pairing (e.g., Seligman, Maier, \& Solomon, 1971). Therefore, if autonomic responding parallels the reported IC, a greater incidence of TOR components is expected after the pertinent slide in comparison to the control slide. Indeed, the pertinent slide was followed by a (marginally significant) greater number of TORs than the control slide.

No difference could be detected between the FIR C-slide and the FIR Tslide. The absence of a differential "conditioned" responding in relation to the on-line expectancies of shock occurrence may be attributed to the small magnitude of the FIR and rapid habituation.

In contrast to the other SCR components, the SIR probability data conflict with the hypothesis that electrodermal responding is congruent with subject's contingency estimates. The SIR is thought to reflect an anticipatory respons to UCS occurrence (see e.g., Prokasy \& Kumpfer, 1973). However, in contrast to our prediction, the SIR component occurred more frequently during the Cslide than during the T-slide. As for the interpretation of this phenomenon it could be speculated -[in line with Ohman's theory (1979)]- that the SIR component in the present study reflects (uncertainty induced) effort "invested" by the central channel in processing CS-UCS contingency information (PE data showed that subjects were more uncertain about shock occurrence after the $\mathrm{C}$-slide than after the T-slide). Of course, the present study is far from conclusive in this respect.

Although the present study provides some evidence for conditioning-like 
autonomic responding associated with on-line and aposteriori covariation estimates, the absence of relevant correlations indicates that there is no simple, linear relationship between the two sets of phenomena. In this respect, present data are consistent with earlier studies which failed to find high positive cognitive-autonomic correlations (e.g., Schiffmann \& Furedy, 1977; see also Merckelbach, van den Hout, \& de Jong, 1989). This led Schiffmann and Furedy to conclude that "there is no clear support for any position which holds that the extent of autonomic conditioning is directly related to the extent of CS-UCS awareness" ( $p$ 276).

In summary, the present experiment showed $l$ that it is possible to induce an IC between a neutral stimulus and an aversive outcome by means of a conditioning-like procedure; 2 that covariation bias can emerge during processing of information concerning CS-UCS contingencies; 3 that an IC can serve to maintain or enhance covariation bias as indexed by on-line probability estimates. Furthermore, the present study provides some support for the hypothesis that a covariation bias is associated with differential autonomic responding. 


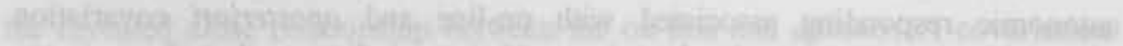

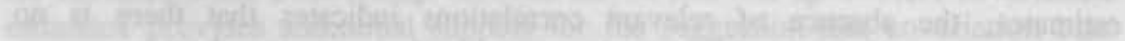

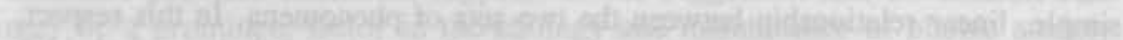

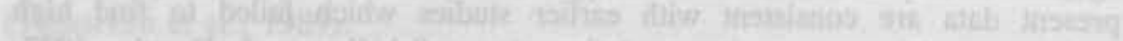

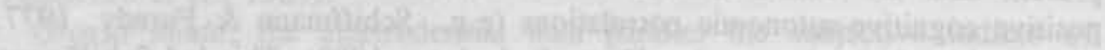

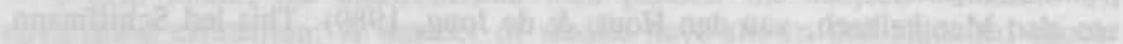

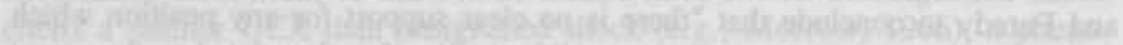

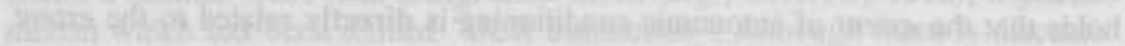

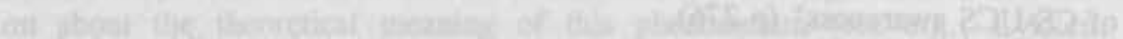

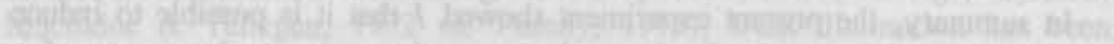

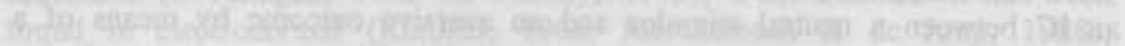

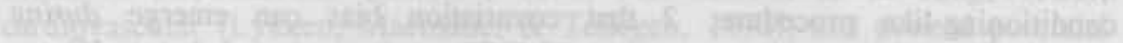

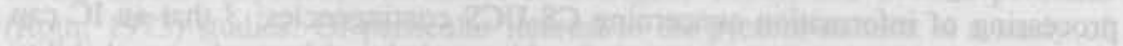

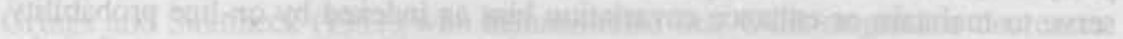

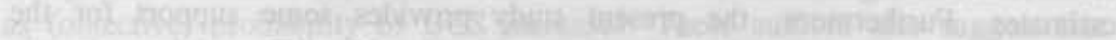

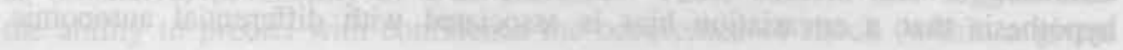

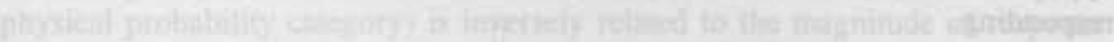

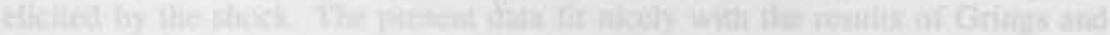

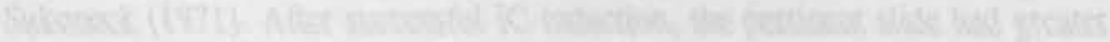

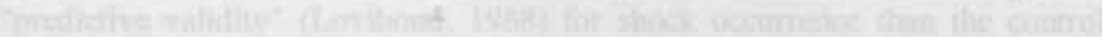

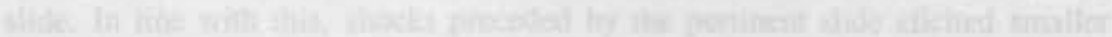

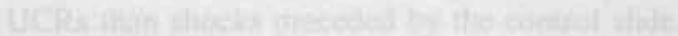

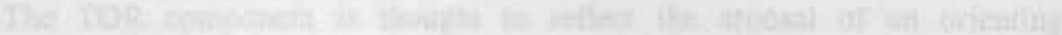

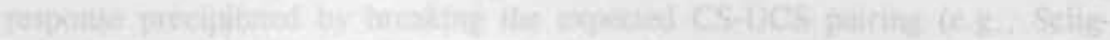

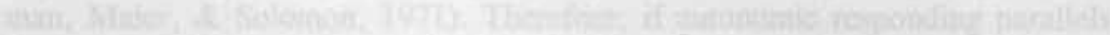

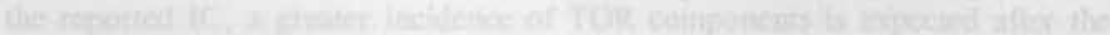

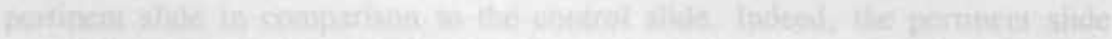

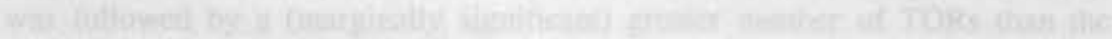

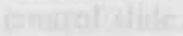

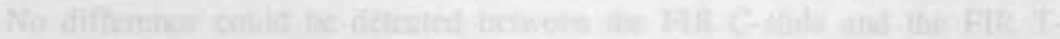

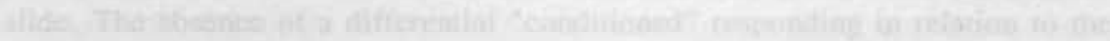

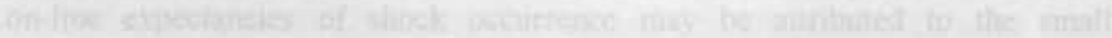

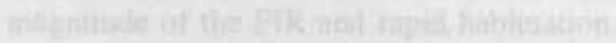

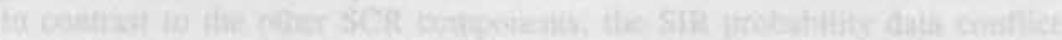

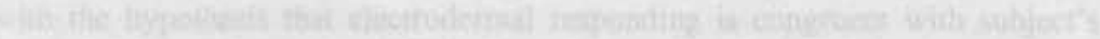

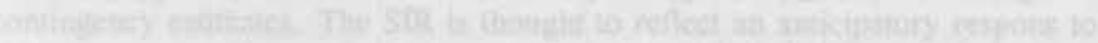

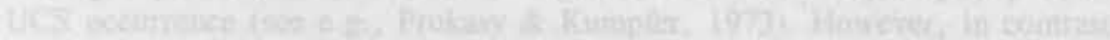
In:

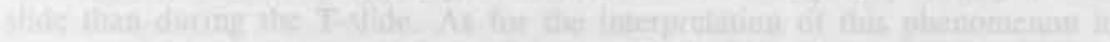

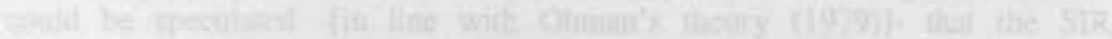

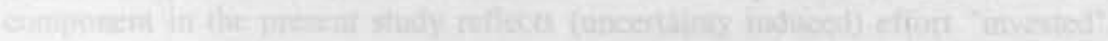

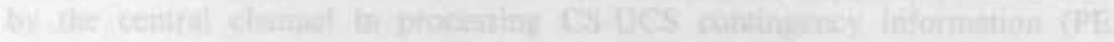

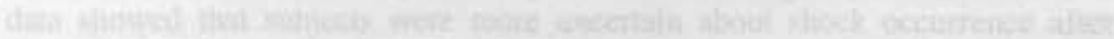

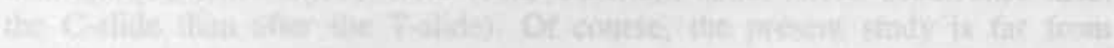

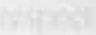



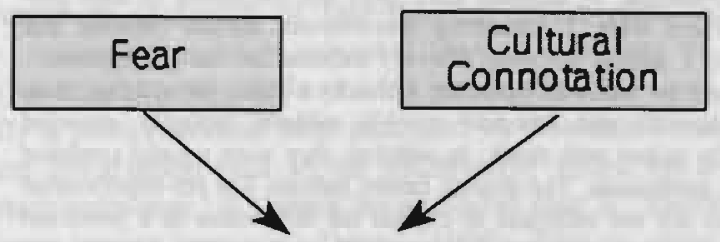

\section{Expectancies}

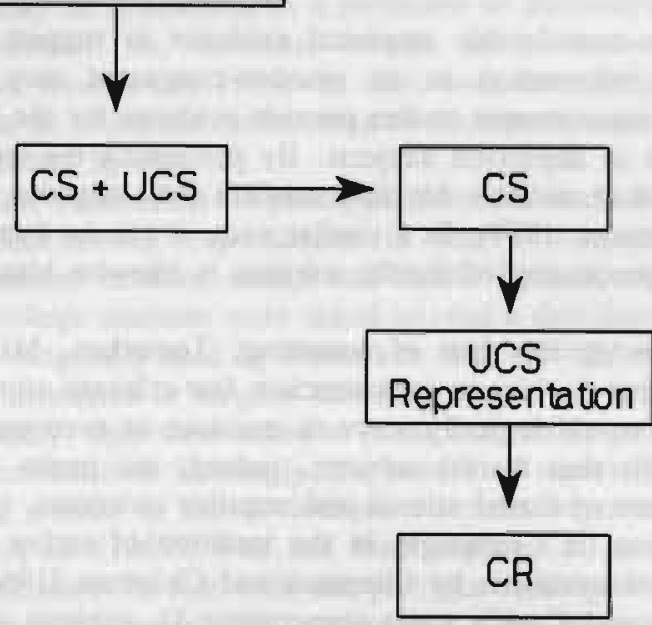

This chapter is published as: De Jong, P.J. (1993). Covariation bias in phobia: Mere resistance to pre-experimental expectancies? Behavior Therapy, 24, 447-454.

Acknowledgements: We thank Professor Joost Bremer, Arie Dijkstra, Heiny Eilkes, and Helga Nauta for their assistance during the data-acquisition. 
Covariation Bias in Phobia: Resistance to Preexperimental Expectancies?

\begin{abstract}
In the present study subjects were asked to read a description of the experimental procedure that was used in a previous study on covariation bias and to imagine that they were about to participate in that experiment. They were then asked to predict the probabilities of all slide/outcome combinations. In addition, they rated the confidence of their predictions. There were 2 concurrent fear-relevant stimuli (weapon and spider) and 1 neutral stimulus (flower) included in the "experiment". Stimuli could be followed by either a shock, a siren, or nothing. Both high-fear $(n=30)$ and low-fear $(n=34)$ subjects typically expected spiders to be paired with shock. In addition, they were equally confident about their predictions. The present results indicate that pre-experimental expectancies are not sufficient to explain the differences in a posteriori reported covariation estimates between high- and low-fear subjects that were found in previous studies.
\end{abstract}

There is considerable empirical evidence to suggest that depressed subjects process information in an emotion-congruent way (e.g., Brewin, 1988). Several experimental studies provide evidence for the operation of a confirmation bias in depressed subjects: By perceiving the world in a more negative fashion than normals do, they confirm their negative mood state (e.g., Alloy \& Abramson, 1979). In a similar vein, it can be hypothesized that the information processing of fearful subjects is likewise biased in a way to confirm fear.

Following this line of reasoning, Tomarken, Mineka, and Cook (1989) argued that "a bias to overassociate fear-relevant stimuli and aversive consequences would logically serve to maintain or even enhance fear." To test the hypothesis that fearful subjects, indeed, are prone to overestimate the cooccurrence of feared stimuli and negative outcomes, they designed an illusory correlation (IC) paradigm in the tradition of earlier research on psychodiagnostic observations by Chapman and Chapman $(1967,1969)$. In Tomarken, Mineka, and Cook's study (experiment 1), subjects with and without fear of snakes (or spiders) were shown an extensive series of slides and outcomes. Three different classes of stimuli were used: slides of snakes or spiders (fearrelevant stimuli), slides of flowers, and slides of mushrooms (neutral stimuli). At slide offset one of three outcomes occurred: an aversive electric shock, a neutral tone, or nothing at all. As it should be in a proper IC-paradigm, all slide/outcome combinations were presented equally often. Postexperimentally, subjects were asked to report the covariation between all slide/outcome combinations. In line with the hypothesis that fearful subjects process information in a fear-confirming way, high-fear subjects especially overestimated the covariation of fear-relevant stimuli and shock.

Employing a similar design, de Jong, Merckelbach, Arntz, and Nijman 
(1992) confirmed that high-fear subjects are especially prone to "covariation bias." Whereas in their studies untreated women who were spider phobics dramatically overestimated the spider/shock association despite the inclusion of concurrent fear-relevant stimuli (i.e., aimed weapons), treated women did not display this overestimation.

A question not empirically addressed so far concerns the origin of the observed IC. The IC might reflect emotion-congruent fear confirmatory information processing during the experiment, as Tomarken and colleagues seem to suggest. In other words, covariation bias results from subjects giving more weight to instances of fear-relevant threat cues (spiders) followed by aversive consequences (e.g., shocks) than to instances of fear-irrelevant cues (flowers or mushrooms) followed by shock. In this view, covariation bias is essentially a computational bias mediated by "on-line emotions." However, as studies on covariation bias so far exclusively relied on a posteriori reported contingency estimates, it cannot be excluded that IC merely reflect preexperimental expectations (see e.g., Davey, 1992). That is, right from the beginning of the experiment subjects may have strong expectations that spiders will be paired with shock, yet fail to correct their expectancy. Thus, in the latter view, covariation bias may be considered as a resistance to disconfirmation of preexperimental expectancies, or, in other words, as a disconfirmation insensitivity (McNally \& Heatherton, 1993). In turn, both the strength of the expectations and the rate at which disconfirmation of preexperimental expectations occur might be affected by fear.

Recently, McNally and Heatherton (in press) performed a study to elucidate the role of pre-experimental expectancies in the post-experimentally reported illusory correlations between snakes and shock. In their study, high and low snake-fearful college students were asked to read a description of the experimental procedure originally used by Tomarken et al. (1989; experiment 1); next, subjects were instructed to imagine that they were about to participate in the experiment; finally, they were asked to predict the likely probabilities of all slide/outcome combinations. Strikingly, the pattern of reported probability estimates was very similar to the covariation estimates reported by the subjects of Tomarken et al. (1989), who had been exposed to an extensive series of slides and outcomes. In line with the previous findings concerning postexperimentally reported covariation estimates, the preexperimental expectation of a strong association between snakes and shock was particular evident in high-fear subjects. These data seem to imply that the covariation bias phenomenon is most parsimoniously explained by a resistance to disconfirmation of preexperimental expectancies. To put it differently, available results do not necessarily imply emotion-congruent information processing.

The present study was undertaken to replicate and extend the findings of McNally and Heatherton (1993). As in the "thought-experiment" of McNally and Heatherton, high-fear and low-fear subjects were asked to read a description of the experimental procedure of a previous IC-experiment (i.e., de Jong et al., 1992). Next, they were asked to rate the probability estimates of all slide/outcome combinations as if they were about to participate in this experi- 
ment. Note that not only the direction of covariation estimates, but also their strength seems to be an important factor in the covariation bias phenomenon. The impact of preexperimental expectancies on covariation detection is thought to increase with their strength (e.g., Alloy \& Tabachnik, 1984). A previous IC-study of de Jong et al. (1992) has shown that postexperimentally reported covariation estimates correlate only very weakly with the reported confidence in these estimates. Therefore, in the present study subjects were asked not only to report probability estimates, but also to report their confidence in these estimates. An additional difference with the McNally and Heatherton study (1993) is that, following the design of de Jong et al. (1992), concurrent danger-related stimuli (i.e., weapons) were added to the fearrelevant (i.e., spiders) and neutral stimuli (i.e., flowers). Furthermore, a concurrent danger-related outcome (i.e., siren) was included instead of a neutral tone. Both, the inclusion of weapons and sirens was aimed at rendering the spider/shock combination less salient, which in turn decreases the likelihood that the reported probability estimates merely reflect the general tendency to associate salient stimuli.

\section{Method}

\section{Subjects}

Subjects were 64 undergraduate students at the department of Health Sciences ( 31 women and 33 men). Mean age was 18.5 years (range 18-22 years). All subjects completed the Spider Phobia Questionnaire (SPQ; Klorman, Weerts, Hastings, Melamed, \& Lang, 1974). The SPQ is a validated 31 item selfreport instrument that measures fear of spiders (Fredrikson, 1983). SPQ scores can range from 0 to 31 . On basis of their scores on the SPQ subjects were post hoc assigned to either the low-fear $(n=34)$ or the high-fear $(n=30)$ group, by means of a median split procedure. The SPQ scores differed significantly between both groups, $\underline{t}(62)=9.4, p<.001$, means being 2.1 and 9.5 , respectively. The mean SPQ score for the high-fear group was well below the SPQ score de Jong et al. (1992) reported for a phobic sample (9.5 vs. 21.9).

\section{Materials and Procedure}

Subjects were presented with a brief description of the illusory correlation experiment by de Jong et al. (1992). The description was a slightly modified Dutch translation of the description originally designed by McNally and Heatherton (1993). After reading the description, subjects completed a modified version of the Covariation Questionnaire (CQ) used in de Jong et al. (1992). That is, in the present study the CQ asks subjects to indicate their probability estimates of all slide/outcome combinations rather than covariation judgments. In addition, it asks subjects to indicate the confidence of their 
estimates. Finally, subjects completed the SPQ. The present study was performed as a part of a larger project. The instructions mimicked those of McNally and Heatherton as close as possible and are presented below.

\section{Thought Experiment}

"This brief questionnaire concerns how people perceive experiments in psychology. You will first be asked to read a description of an experiment, and then to imagine yourself as a subject in the experiment."

\section{Description of the Experiment}

"In this experiment, you'll be asked to sit in a comfortable reclining chair and view a series of slides that will be projected on a screen in the laboratory. There will be three categories of slides: pictures of flowers, pictures of spiders, and pictures of weapons. Any given slide will be followed by one of three possible outcomes. You will either hear a short siren, or you'll feel a harmless shock on your forearm, or you'll experience nothing (Before we start the actual experiment, we will ask you to select a level of shock that is definitely uncomfortable, but not painful.) Pay close attention to what is happening because your task is to determine whether or not there is a relationship between any category of slide and any of the outcomes following the slide. Once again, focus in on whether there is a relationship between different categories of slides and the three outcomes.

Imagine that you are about to participate in this experiment. Please answer the following questions as if you were about to participate in this experiment."

After reading this description, subjects completed the $\mathrm{CQ}$. For these estimates, $100-\mathrm{mm}$ visual analogue scales (VAS) were used ranging from $0 \%$ to $100 \%$.

\section{Data Reduction and Analysis}

Probability estimates. Because between group differences were only expected for a limited set of reported probability estimates, a Group (high-fear/lowfear) X Stimulus (spider/weapon/flower) X Outcome (siren/shock/nothing) analysis of variance is likely to lack sufficient sensitivity to detect the predicted effects. Therefore, in line with earlier studies on covariation bias (de Jong et al. 1992; Tomarken et al. 1989), only the contrasts that are most relevant to the experimental predictions were subjected to a set of $a$ priori t-tests in order to evaluate whether subjects specifically expected the spider slides to be followed by shock: spider/shock versus flower/shock, spider/shock versus weapon/shock, spider/shock versus spider/siren, and spider/shock versus spider/nothing. To control for experimentwise error alpha was set at $.05 / 4=.0125$ (see Tomarken et al. 1989 for a detailed justification for this data-analytic strategy). In addition, it was tested whether high-fear and low- 
fear subjects differed in regard to their spider/shock probability estimates. Finally, to explore the influence of saliency per se (irrespective of prior fear) on subjects' preexperimental expectancies, the weapon/siren versus spider/shock probability estimates were subjected to within-subjects t-tests.

Confidence ratings. The means of the reported confidence ratings for each of the relevant stimulus/outcome combinations, were subjected to a betweengroups t-test to evaluate whether high-fear and low-fear subjects differed in respect to the confidence in their probability estimates.

\section{Results}

Most relevant probability estimates are depicted in Figure 3.3. The spider/shock probability estimates of the high-fear subjects were significantly greater than their flower/shock probability estimates, $\mathrm{t}(29)=7.84, \mathrm{p}<.001$. The comparison between the spider/shock and the weapon shock estimates reached borderline significance, $\mathrm{t}(29)=2.63, \mathrm{p}=.014$.

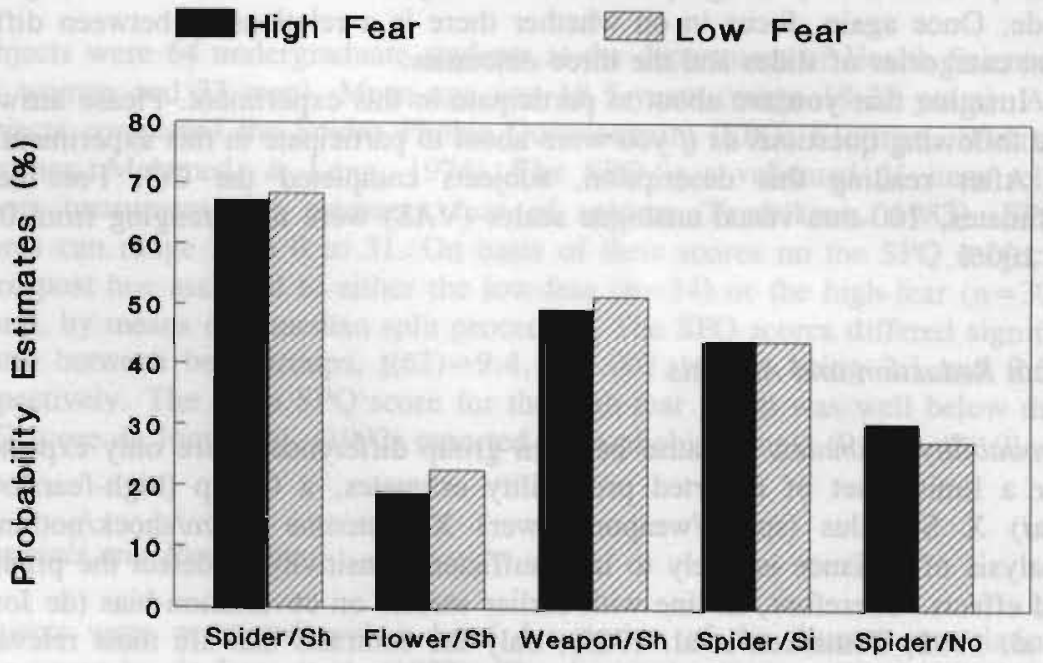

Figure 3.3: Most relevant a priori reported probability estimates. $(s h=s h o c k ;$ si $=$ siren; $n o=n o$ outcome)

In addition the spider/shock estimates were greater than both the spider/nothing and the spider/siren probability estimates, $\mathrm{t}(29)=4.93, \mathrm{p}<.001$, and $\mathrm{t}(29)=3.00, \mathrm{p}<.001$, respectively. 
An identical pattern of results was evident in the low-fear subjects. The spider/shock probability estimates were significantly greater than the spi$\mathrm{der} /$ nothing and spider/siren estimates, $\mathrm{t}(33)=7.82, \mathrm{p}<.001$, and $\mathrm{t}(33)=4.10$, $\mathrm{p}<.001$, respectively. The comparison of the spider/shock and the flower/shock estimates differed significantly, and the comparison between the spider/shock and the weapon/shock probability estimates reached borderline significance, $\mathrm{t}(33)=7.36, \mathrm{p}<.001$, and $\mathrm{t}(33)=2.53, \mathrm{p}=.016$, respectively. As is evident from Figure 3.3, the spider/shock probability estimates were equal for both groups, $\mathrm{t}(62)<1$. For high- as well as low-fear subjects, the weapon/siren estimates were not significantly different from the spider/shock probability estimates, $\underline{\mathrm{t}}(29)=-0.95, \mathrm{p}>.15$ and $\mathrm{t}(33)=1.45, \mathrm{p}>.15$, respectively.

Confidence ratings. Mean confidence ratings for the relevant slide/outcome combinations did not differ between groups, $\mathrm{t}(62)<1$, means being 52.2 for the high-fear subjects and 49.6 for the low-fear subjects.

\section{Discussion}

The present findings clearly indicate that despite the inclusion of a concurrent danger-related stimulus (weapon) and a concurrent danger-related outcome (siren), subjects still show the preexperimental expectancy that typically spiders will be paired with shock. The present result that subjects' spider/shock probability estimates did not differ from the weapon/siren estimates suggests that saliency per se, irrespective of prior fear, might be an important determinant of subjects' a priori expectancies. This suggestion is underlined by the finding that the spider/shock expectancy bias was evident in both high-fear and low-fear subjects. In addition, both groups of subjects were equally confident in respect to their reported probability estimates. In other words, the present data indicate that high-fear and low-fear subjects enter ICexperiments with an equally strong expectation that spiders, especially, will be associated with shock.

The latter finding is somewhat at variance with the results presented by McNally and Heatherton (1993). They also found a similar expectancy bias for high- and low-fear subjects. However, in their study, low-fear subjects' snake/shock predictions were smaller than those of high-fear subjects. Note that the high-fear subjects in the McNally and Heatherton study were snakerather than spider-fearful college students and possibly were more fearful than the high-fear subjects in the present study (mean snake-fear score was 4.9 on a scale from 0 ["none"] to 6 ["terror"]). Yet, the spider/shock estimates of the high-fear subjects in the present study were highly similar to those of the high-fear subjects in the McNally-Heatherton study (approx. $69 \%$ vs $74 \%$ ). In other words, the failure to find differences between the spider/shock estimates of high- and low-fear subjects in the present study was not due to relatively low spider/shock estimates of the high-fear subjects, but to relatively high spider/shock expectancies of the low-fear subjects ( $70 \%$ vs $50 \%$ in the McNally 
and Heatherton study). Apparently, the increased ambiguity of the present study, resulted in elevated spider/shock expectancies for both high- and lowfear subjects.

The pattern of the reported slide/outcome expectations in the present study is highly similar to that of the postexperimentally reported covariation estimates of the high-fear subjects in the de Jong et al. (1992) study. This finding corroborates the conclusion previously drawn by McNally and Heatherton (in press) that "biased covariation estimates might be at least partly attributed to pre-experimental expectancy biases, and not arise solely from biased on-line processing". Yet, the present finding that the preexperimental expectancies were similar and equally strong for both high-fear and low-fear subjects suggests that postexperimentally reported illusory correlations not merely reflect preexperimental expectancies. In the de Jong et al. (1992) study (that actually performed the described experiment), only high-fear subjects showed a bias to overassociate spiders with shock, while low-fear subjects were in fact quite accurate. Taking the results of both studies together, these results suggest that the biased preexperimental spider/shock expectancies of high-fear subjects are especially resistant to disconfirmation. Because high-fear and low-fear subjects appear to have an equally strong preexperimental expectancy bias, processes during the actual experimental procedure are apparently responsible for the between-groups differences regarding the postexperimentally reported covariation estimates. Further research including both pre- and post-experimental covariation assessment is one way to test directly the speculation that prior fear affects the rate at which disconfirmation of preexperimental expectancies occurs rather than the strength of the preexperimental epectancies per se.

To summarize, both high-fear and low-fear subjects typically expected spiders to be paired with shock. In addition, they were equally confident about their predictions. Therefore, the present results suggest that preexperimental expectancy bias is not sufficient to explain the differences in a posteriori reported covariation estimates between high- and low-fear subjects that were found in previous studies. Meanwhile, the present results are in line with the hypothesis that covariation bias arises from a resistance to disconfirmation of preexperimental expectancies. The available data suggest that the insensitivity to disconfirmation is at least partially mediated by fear. In other words, the available data do provide some indirect evidence for emotion-congruent information processing in fearful subjects. 


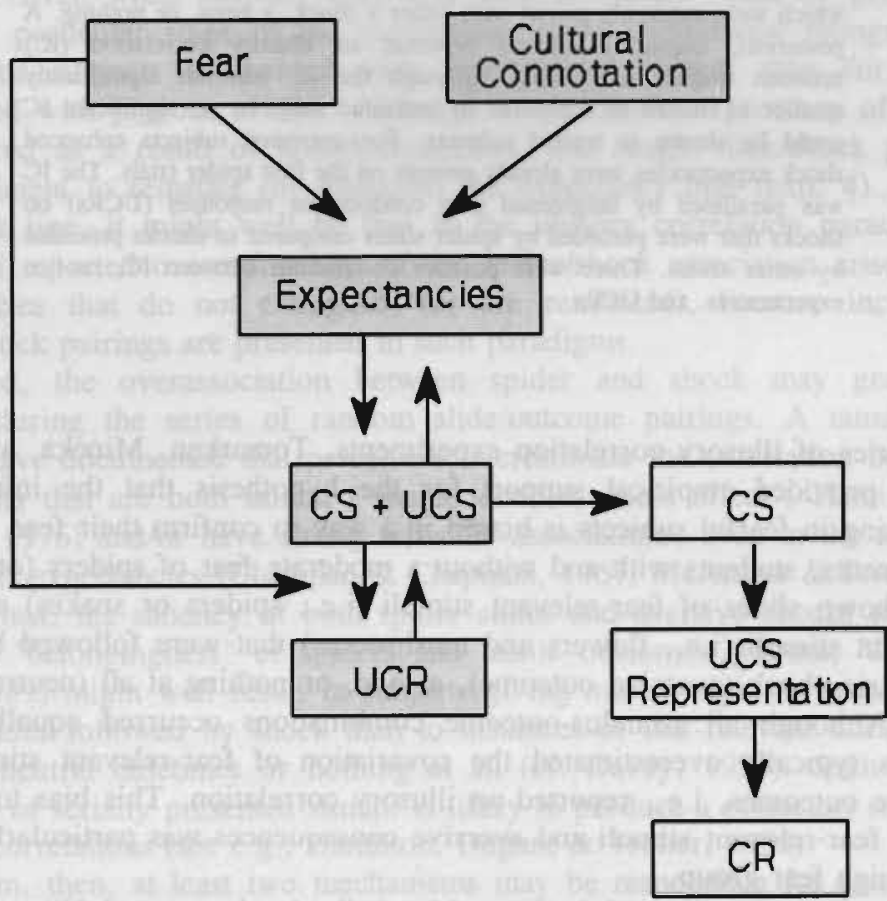

This chapter is based on a paper presented at the 34.Tagung der experimentell arbeitender Psychologen (Münster, 1992) and a paper presented at the 2nd European Congress on Psychophysiology in Berlin (1992). A modified version of this chapter is published as: De Jong. P.J., Merckelbach, H., \& Arniz, A. (1994). Covariation bias in phobic subjects: The relationship between apriori expectancy, on-line expectancy, autonomic responding, and a posteriori contingency judgment. Journal of Abnormal Psychology, in press.

Acknowledgements: We thank the therapists Arie Dijkstra, Birgit Mayer, Edith Lavy, Anja Meijboom, and Dorien Wolfs. In addition, we are grateful to Dr. Karel Eisses, who (again) kindly provided Drosophila for our spiders. Furthermore, we thank Annie Raven for her assistance during acquisition and work up of the follow-up data. 
Covariation Bias in Phobic Subjects:

\title{
The relationship between a priori expectancy, on-line expectancy, autonomic responding, and a posteriori contingency judgment.
}

\begin{abstract}
Nineteen untreated and 19 treated spider phobics were exposed to a series of 72 slides comprising pictures of spiders, weapons, and flowers which were randomly paired with either a shock, a siren, or nothing. A posteriori, untreated subjects reported an illusory correlation (IC) between spiders and shock. Although the IC was not significantly smaller in treated as compared to untreated subjects, no significant IC could be shown in treated subjects. For untreated subjects enhanced shock expectancies were already present on the first spider trials. The IC was paralleled by heightened skin conductance responses (UCRs) on shocks that were preceded by spider slides compared to shocks preceded by other slides. There were positive correlations between IC, on-line expectancies, and UCRs.
\end{abstract}

In a series of illusory correlation experiments, Tomarken, Mineka, and Cook (1989) provided empirical support for the hypothesis that the information processing in fearful subjects is biased in a way to confirm their fear. In these experiments, students with and without a moderate fear of spiders (or snakes) were shown slides of fear-relevant stimuli (i.e., spiders or snakes) and fearirrelevant stimuli (i.e., flowers and mushrooms) that were followed by either an electric shock (aversive outcome), a tone, or nothing at all (neutral outcomes). Although all stimulus-outcome combinations occurred equally often, subjects typically overestimated the covariation of fear-relevant stimuli and aversive outcomes, i.e., reported an illusory correlation. This bias to overassociate fear-relevant stimuli and aversive consequences was particularly strong in the high fear group.

Subsequent research of de Jong, Merckelbach, Arntz, and Nijman (1992) confirmed that initial fear can be an important determinant of such a covariation bias. In that study it was demonstrated that whereas untreated spider phobics specifically overestimated the covariation between spider slides and shock outcome, treated subjects did not show a significant bias to overassociate phobia-relevant cues and aversive outcomes. The costs of such a covariation bias seem obvious: Threat associated with the phobic stimuli is confirmed and in turn the heightened threat might maintain or even enhance phobic fear (e.g., de Jong, van den Hout \& Merckelbach, 1994; Tomarken et al. 1989).

Although the a posteriori reported illusory correlation between fearrelevant stimuli and aversive outcomes has proven to be a fairly robust phenomenon, the underlying factors are largely unknown. At least two mechanisms may be relevant in this context. First, subjects may enter the 
experiment with the strong expectation that specifically spiders will be followed by shock and then fail to correct their expectancy (cf. McNally \& Heatherton, 1993). Previous research clearly shows that strong a priori expectancies can result in expectancy-congruent illusory correlations, especially in case the available situational information is ambiguous (e.g., Alloy \& Tabachnik, 1984).

Germane to this issue is a series of experiments by Davey (1992). In this study (exp. 1), undergraduate students were exposed to slides of fear-relevant stimuli (spiders and snakes) or to slides of fear-irrelevant stimuli (cats and pigeons). Even when subjects were told that no shocks would be presented during a particular stage of the experiment, shock expectancy ratings were higher for subjects confronted with fear-relevant stimuli than for those confronted with pigeons or cats. Although the enhanced expectancy of shock disappeared as a result of nonreinforcement, one single slide/shock pairing was sufficient to reinstate (or maintain) the expectancy bias (exp. 4). Following this line, it might well be that in the illusory correlation paradigm a posteriori reported overestimations of the spider/shock association arise from expectancies that do not extinguish (or are reinstated), because incidental spider/shock pairings are presented in such paradigms.

Second, the overassociation between spider and shock may gradually emerge during the series of random slide/outcome pairings. A number of studies have documented that perceivers overestimate the correlation between two events that are both salient ("shared distinctiveness effect"; Hamilton \& Gifford, 1976) and/or have strong semantic associations, even in the absence of a priori expectancies (Chapman \& Chapman, 1967; McArthur \& Friedman, 1980). Thus, the saliency of both spider slides and aversive shocks together with the "belongingness" of spiders and tactile outcomes (Hamm, Vaitl, \& Lang, 1989) might well result in subjects giving more weight to instances of spider slides followed by shock than to instances of fear-relevant cues followed by neutral outcomes or nothing at all (cf. Davey, 1993). Such biased encoding of serially presented stimuli is likely to produce a posteriori reported illusory correlations (see e.g., Hamilton, Dugan, \& Trolier, 1985).

In sum, then, at least two mechanisms may be responsible for generating the a posteriori reported illusory correlation between fear-relevant stimuli and aversive outcomes: Expectancy bias and encoding bias. Of course, these biases are not mutually exclusive and may well act in concert. Furthermore, there is evidence to suggest that high levels of prior fear might intensify both expectancy bias (McNally \& Heatherton, 1993; Diamond, Matchett, \& Davey, 1993) and encoding bias (cf., Mackie, Hamilton, Schroth, Carlisle, Gersho, Meneses, Nedler, \& Reichel, 1989).

Most previous studies concerning illusory correlation and anxiety predominantly relied on a posteriori reported estimates. Consequently, it is largely unknown to what extent the mechanisms described above are at work in the illusory correlation paradigm. The present study attempted to investigate whether differential preexperimental expectancies and differential encoding during the experiment underlie the illusory correlation phenomenon. Therefo- 
re, the present study was designed to seperately monitor a priori expectancies, on-line processing at the cognitive and physiological level, and a posteriori contingency judgment. A second aim of the present study was to replicate the effect of treatment on illusory correlation (de Jong et al., 1992) and to explore its mediating factors.

The experimental procedure of the present study was highly comparable to that employed in the de Jong et al. (1992) study. Again, subjects were treated and untreated women who were severe spider phobics. Alternative dangerrelated stimuli (i.e., aimed weapons) were added to the phobia-related stimuli (i.e., spiders) and positive stimuli (i.e., flowers). In addition, an alternative danger-related outcome (i.e., a siren) was included instead of the neutral tone presented in earlier studies. Both the inclusion of sirens and the inclusion of aimed weapons may render the spider/shock combination less salient, which in turn decreases the likelihood that reported illusory correlations merely reflect the general tendency to overestimate the covariation of salient (danger-related) stimuli (see e.g., Hamilton \& Gifford, 1976; but see also Tomarken et al., 1989, exp. 2).

\section{Method}

\section{Subjects}

Subjects were 40 women who were spider phobics (mean age $=26.8$ years, range $=16-45$ years). They applied for treatment after reading articles in magazines and newspapers about previous "spider-phobia-projects" at Limburg University. Only subjects who indicated that fear of spiders strongly interfered with daily life and who suffered from an isolated fear were invited to participate in research. All subjects met criteria from the Diagnostic and Statistical Manual of Mental Disorders (rev. 3rd ed.; American Psychiatric Association, 1987) for simple phobia. These diagnoses were made by an experienced psychotherapist (A.A.). Subjects participated in the experiment in return for free treatment. One group of subjects $(n=21)$ was tested before treatment, and a second group of subjects $(n=19)$ was tested after treatment. During the experiment two subjects of the pretreatment group panicked and preferred to withdraw from the experiment. These subjects were excluded from the analyses, leaving 19 subjects in the pretreatment group. The mean scores on the Spider Phobia Questionnaire (SPQ; Klorman, Weerts, Hastings, Melamed, \& Lang, 1974) for the untreated and treated group were 21.8 and 9.2, respectively. Before treatment, SPQ scores were equal for both groups (21.8). SPQ scores were comparable to the mean scores that Fredrikson (1983) and Arntz and Lavy (1992) reported for their spider phobic samples. Subjects also underwent a behavioral approach test (BAT), both before and after treatment. The BAT was scored on a 13-point scale ranging from 0 (distance to spider $300 \mathrm{~cm}$ ) to 12 (spider on hand). Before treatment the BAT scores were similar for the pretreatment and posttreatment groups ( 4.0 and 4.7 , respectively). The 
treated subjects strongly benefitted from therapy as indexed by SPQ scores $(\mathrm{t}(18)=-8.7, \mathrm{p}<.001 ; \underline{\mathrm{M} s}=21.8$ and 9.2$)$, and by BAT scores $(\mathrm{t}(18)=14.2$, $\mathrm{p}<.001 ; \underline{\mathrm{M}} \mathrm{s}=4.0$ and 11.1 ).

\section{Apparatus and Stimulus Materials.}

Three categories of slides were used: four different slides depicting flowers, four different slides depicting spiders, and four different slides depicting weapons (knives and guns directed toward the subject; e.g., Hughdahl \& Johnsen, 1989). The slides were projected onto a white screen $(80 \mathrm{~cm} \times 120$ $\mathrm{cm}$ ), approx. $3 \mathrm{~m}$ in front of the subject. A Kodak Carousel was used for stimulus presentation. Three types of outcomes occurred during the experiment: a 1-s shock, a $1-\mathrm{s}$ siren $(5 \mathrm{~Hz})$, or nothing. Electric shocks $(\mathrm{dc})$ were delivered from a specially designed shock generator $(0-40 \mathrm{~mA})$ and administered to the subjects' lateral side of the upper right arm through two electrodes ( $8 \mathrm{~mm}$ diameter; $\mathrm{Ag} / \mathrm{AgCl}$ ). Sirens were delivered by a tone generator connected to a loudspeaker that was located at the left side of the subjects. For measuring on-line outcome expectancies, a pointer lever was used. The pointer could be rotated through $180^{\circ}$ Horizontal left (pointing to the loud speaker), vertical, and horizontal right (pointing to the shock electrodes) positions represented siren, nothing, and shock, respectively. During the experiment, skin conductance was measured by bipolar placement of $(8-\mathrm{mm}$ diameter; $\mathrm{Ag} / \mathrm{AgCl}$ ) electrodes to the medial phalanges of the third and fourth finger of the non-dominant hand, using the method of constant voltage $(0.5$ V). The electrodes were filled with an isotonic paste following the recommendations of Fowles, Christie, Edelberg, Grings, Lykken, and Venables (1981) and connected to a Beckman skin conductance coupler (type 9844). The coupler allowed for a maximum sensitivity of 0.05 microSiemens. Stimulus presentation, delivery of sirenes and shocks, and intertrial intervals were controlled by a PDP Minc-II laboratory computer.

\section{Procedure}

Subjects were randomly assigned to one of two groups. Subjects in the posttreatment group first underwent a 2.5 -hr one-session in vivo exposure (as described by Öst, 1989) followed by a 1.5 -hr booster session after one week, before they participated in the experiment. Subjects in the pretreatment group participated in the experiment before they received exposure therapy. The one-session treatment employed consisted of hierarchical exposure in vivo and modeling and has been found to yield good immediate and long term results (Muris, de Jong, Merckelbach, \& van Zuuren, in press; de Jong, Arntz, \& Merckelbach, 1993; Arntz \& Lavy, 1992; Öst, 1989).

During the experiment, subjects were seated in a sound attenuated room. A one-way screen separated the experimental and the registration room. The 
subject was told that it was her task to determine the relationship between categories of slides and outcomes. She was instructed to indicate outcome expectancies during each slide presentation by means of the pointer. Then electrodes were attached. Next, shock intensity level was determined using a shock work-up procedure. Stepwise, electrical current was increased until the subject indicated that the shock was definitely uncomfortable but not painful. A similar shock intensity level was reached for treated and untreated subjects, means being $3.4 \mathrm{~mA}$ and $3.1 \mathrm{~mA}$, respectively $(\mathrm{t}(37)<1)$. After the subject had confirmed that the task was clear to her, she was left alone and the lights were dimmed. Subjects were exposed to 72 slides each of 8-s duration. Three different categories were used: flowers (neutral stimuli), spiders (phobiarelevant stimuli), and weapons (alternative fear-relevant stimuli). One of three possible outcomes occurred at slide disappearance: a 1-s shock (aversive outcome) or a 1-s siren (alternative danger-related outcome) or nothing at all (neutral outcome). Across all trials, the conditional probability of any outcome given the prior occurrence of any category of slide was $1 / 3$ and so was the probability of occurrence of each category of slide and each type of outcome. Intertrial intervals ranged from 10 to $30 \mathrm{~s}$. Stimulus-outcome combinations were randomly distributed across trials, with the restriction that on two successive trials no identical stimulus-outcome combinations occurred.

To cancel out order effects, three different sequences were used. At the end of the experiment, subjects completed the Covariation Questionnaire (CQ). The CQ asks subjects to estimate the percentage of occurrence of each outcome given the prior occurrence of each slide type. For these estimates, $100-\mathrm{mm}$ visual analogue scales (VASs) were used ranging from $0 \%$ to $100 \%$.

\section{Data Reduction and Analysis}

To reduce type I error, for each response set (i.e., a posteriori contingency estimates, on-line expectancies and skin conductance responses) alphas were Dunn-Bonferroni corrected. Within and between groups comparisons were considered separately in this respect.

A posteriori reported contingency estimates. Because both within and between group differences were only expected for a limited set of reported contingency estimates, a Group (treated/untreated) X Stimulus (spider/weapon/flower) X Outcome (shock/siren/nothing) analysis of variance is likely to lack sufficient sensitivity to detect the predicted effects. Therefore, in line with earlier studies on covariation bias (McNally \& Heatherton, 1993; de Jong, 1993; de Jong et al., 1992; Tomarken et al., 1989), only the contrasts that are most relevant to the experimental predictions were subjected to a set of a priori ttests to retain sufficient power. It was evaluated (a) whether the spider/shock contingency estimates differed from the weapon/shock, flower/shock, spider/tone, and spider/nothing contingency estimates and (b) whether the pattern of contingency estimates differed between the pre- and posttreatment group, 
that is, whether the reported spider/shock relative to the pooled weapons and flowers (non-phobic)/shock contingency differed between groups. To control for responsewise error, alpha was set at $.05 / 4=.0125$ for the within groups comparisons.

Initial expectancies. To investigate initial expectancies, the number of subjects in both groups that indicated to expect a shock on the first trial of each class of slides was computed. For both the untreated and the treated group, initial expectancy data were subjected to 2 Sign tests. The following within groups contrasts were tested: (a) the first spider slide versus the first weapon slide, and (b) the first spider slide versus the first flower slide. Finally, to investigate between groups differences, a Chi-square test was conducted to evaluate the difference between the untreated and treated group with respect to the number of subjects expecting a shock after the first spider slide $\left(\mathrm{H}_{\mathrm{o}}\right.$ : $\left.\mathrm{Pie}_{\mathrm{pre}}=\mathrm{Pie}_{\mathrm{post}}\right)$.

For both within groups comparisons alpha was set at $.05 / 2=.025$.

On-line expectancies. For each type of slide the number of trials was computed on which subjects indicated to expect a shock outcome, in blocks of 4 trials. The on-line expectancy data were subjected to a MANOVA trend analysis. Zero and first order trends (overall mean and linear trend, respectively) were analyzed. The following within groups contrasts were tested for both treated and untreated subjects: spider trials versus flower trials, and spider trials versus weapon trials. Before entering the analysis, for each block of trials appropriate contrasts were computed (i.e., spider minus flower and spider minus weapon). After this computation, the mean trend was analyzed to test whether spiders were more often expected to be followed by shock than both weapons and flowers. The linear trend was inspected to evaluate whether the spider/shock expectancies changed differently than both the flower/shock and weapon/shock expectancies during the experiment. In addition, a between groups factor was included to test whether treated and untreated subjects differed regarding the spider/shock expectancies relative to the pooled weapon and flower shock expectancies. Alpha was set at $.05 / 4=.0125$ for the within groups comparisons and at $.05 / 2=.025$ for the between groups contrasts.

Skin conductance response. Two different skin conductance responses were scored: The first interval response (FIR; $1-4 \mathrm{~s}$ after slide onset for each trial) and the unconditioned response (UCR; SCR 1-4 s after shock onset). A response was considered to be an upward curve of the skin conductance response; The magnitude of the response was obtained by measuring the distance between the trough and the apex of the curve. Before entering the analyses, electrodermal responses (magnitudes) were square-root transformed to normalize the data. FIRs and UCRs were analyzed by means of MANOVA trend analysis. Two trends were inspected: The mean trend (reflecting the average response) and the lineair trend (as an index of habituation). The following within group contrasts were tested: spider trials versus flower trials and spider trials versus weapon trials. It was also tested whether there were 
group differences (treated vs. untreated) with regard to the spider trials as compared to the pooled flower and weapon trials. For the within and between groups comparisons alpha was set at $.05 / 4=.0125$ and $.05 / 2=.025$, respectively.

\section{Results}

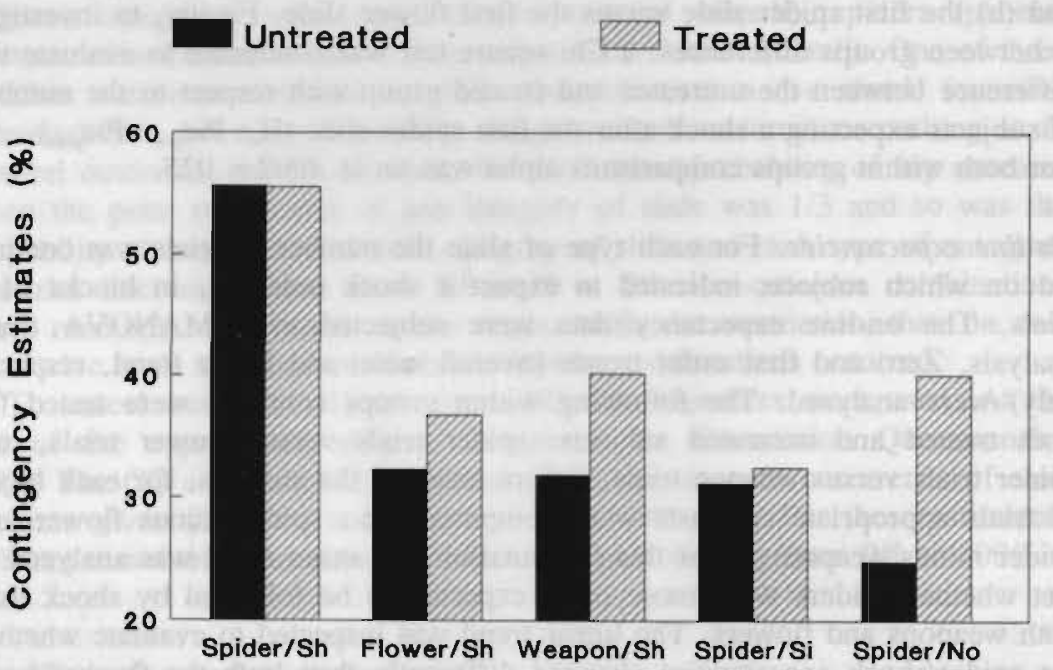

Figure 3.4: Most relevant a posteriori reported contingency estimates of the treated and untreated spider phobics. (sh=shock; $10=$ siren; no=no outcome.)

Covariation estimates (a posteriori). The untreated spider phobics specifically overestimated the spider/shock association: All planned comparisons reached significance. The spider/shock contingency estimates of untreated subjects were significantly higher than both their weapon/shock, $\underline{t}(18)=4.0, \underline{\mathrm{p}}<.0125$, and flower/shock, $\mathrm{t}(18)=2.85, \mathrm{p}<.0125$, contingency estimates. Additionally, the spider/shock contingency estimates were significantly higher than the spider/siren, $\underline{\mathrm{t}}(18)=2.80, \mathrm{p}<.0125$, and the spider/nothing, $\mathrm{t}(18)=4.49$, $\mathrm{p}<.0125$, contingency estimates. In treated subjects the specific overassociation of the spider/shock combination did not reach significance. That is, their spider/shock estimates were not significantly different from their spider/nothing, spider/siren, and weapon/shock estimates. Only the planned comparison between the spider/shock and flower/shock contingency estimates reached borderline significance, $\underline{t}(18)=2.73, p=.014$. For all other comparisons, ts $(18)<2.0$, ps $>.05$.

Additional analyses revealed that the spider/shock and the pooled weapon- 
flower/shock estimates differed significantly for the untreated subjects, $\mathrm{t}(18)=3.7, \mathrm{p}=.002$. For the treated subjects, this contrast differed only marginally significantly from zero, $\mathrm{t}(18)=2.0, \mathrm{p}=.06$. There was no between groups difference in this respect, $\mathrm{t}(36)=1.5, \mathrm{p}>.10$.

\section{Initial expectancy.}

A sign-test indicated that untreated subjects more often expected a shock after the first spider slide $(78 \%)$ and first weapon slide $(72 \%)$ than after the first flower slide $(11 \%)$, ps $<.01$. The number of treated subjects that expected a shock after the first spider slide $(37 \%)$ was not significantly different from the number of treated subjects that expected a shock after the flower (16\%) or weapon slide $(63 \%)$, ps $>.20$. Treated subjects more often expected a shock after the first weapon slide $(63 \%)$ than after the first flower slide (16 $\%), \mathrm{p}=.02$. A Chi-square test revealed that significantly more untreated (78 $\%)$ than treated (37\%) subjects expected a shock after the first spider slide, $\mathrm{X}^{2}(1)=6.3, \mathrm{p}=.012$.

\section{On-line expectancy}

The on-line shock expectancies are depicted in Figure 3.5. A MANOVA trend analysis revealed a significant multivariate effect of slide type for untreated subjects $\underline{F}(4,33)=6.4, \underline{p}<.01$. The univariate tests indicated that untreated subjects expected shock more often after spider slides than after flower slides, $\underline{F}(1,36)=10.7, p=.002$. The difference between spider slides and weapon slides reached borderline significance, $\underline{F}(1,36)=5.2, p=.03$. There were no significant differences regarding linear trends among the three different stimuli (ps $>.045$ ). Thus, by and large, untreated subjects more often expected shocks after spider slides than after both weapon and flower slides, but the development of on-line shock expectancies over trials did not significantly differ between slides.

For treated subjects the multivariate effect of slide type reached significance, $\underline{F}(4,33)=3.32, p=.021$. Univariate $\underline{F}$ tests testing the a priori contrasts were N.S.; However, there seemed to be one trend, in that treated subjects tended to expect more often a shock after spider slides than after flower slides, $\mathrm{F}(1,36)=3.9, \mathrm{p}=.055$. None of the other univariate tests reached significance $(\underline{F} s<2, \underline{p s}>.20)$.

The proportion of trials on which subjects indicated to expect a shock after spider slides relative to that during pooled weapon and flower slides was not significantly larger for untreated as compared to treated subjects, $\underline{F}(1,36)=1.1, p=.30$. In addition, the linear trends were not significantly different between groups, $\mathrm{F}(1,36)=1.1, \mathrm{p}=.30$. In other words, the overall pattern of on-line expectancies was not significantly different between treated and untreated subjects. 
Treated Subjects

- Spider $\rightarrow$ Weapon $\rightarrow$ Flower

- Spider

Untreated Subjects

든
5
을
E
$\frac{3}{2}$
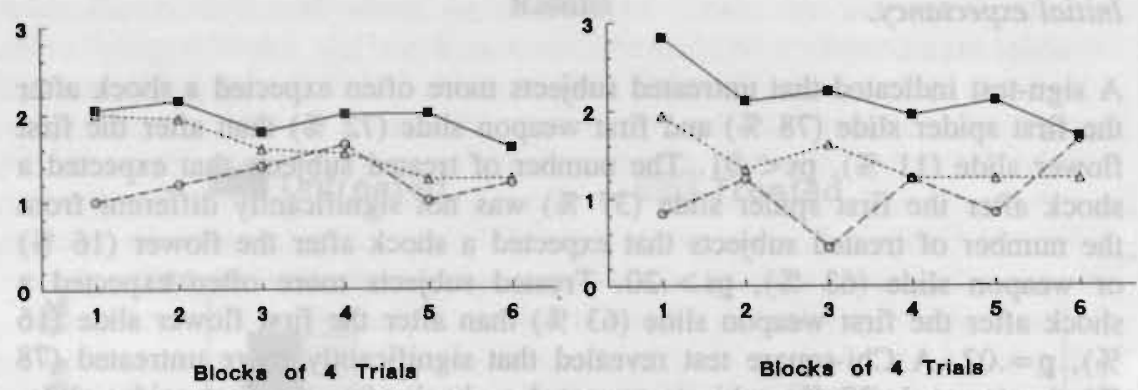

Figure 3.5: On-line reported excpectancies of shock outcome. Expectancies are expressed in the mean number of trials on which subjects indicated to expect a shock (blocks of 4 trials; 4 means shock expected after each slide of a certain class; $11 / 3$ reflects random outcome selection). The right and left panel depict expectancy data of treated and untreated subjects, respectively.

\section{Skin conductance response}

The upper panel of Figure 3.6 shows the FIRs. A MANOVA trend analysis revealed a significant multivariate effect of slide type for untreated subjects $\underline{F}(4,33)=8.0, \underline{p}<.01$. The univariate tests indicated that untreated subjects reacted with larger FIRs to spider slides than both flower and weapon slides, $\underline{F}(1,36)=27.3, \mathrm{p}<.01$, and $\underline{E}(1,36)=24.6, \underline{\mathrm{p}}<.01$, respectively. In addition, there was a significant difference in first order trends between spider and flower slides, $\underline{E}(1,36)=12.1, \mathbf{p}<.01$. Furthermore, a marginally significant difference was found with respect to first order trends of spider and weapon slides, $\mathrm{F}(1.36)=5.4, \mathrm{p}=.026$. Thus, there was evidence for relatively slow habituation to phobic slides.

For treated subjects, no multivariate effect of slide type was found, $\underline{F}(4,33)<1$. Univariate $\mathrm{E}$ tests showed that the FIRs of treated subjects did not differ among slides $(\mathrm{Fs}(1,36)<2.0$, ps $>.15)$. The habituation pattern was also similar for all stimuli. This is reflected in the absence of a difference in linear trends between the three slides $(\mathrm{Fs}(1,36)<1.5$, ps $>.20$ ).

A MANOVA trend analysis revealed that the difference between FIRs on spider trials and FIRs on the pooled flower and weapon trials was larger for untreated than for treated subjects, $F(1,36)=3.8, \underline{p}<.05$. Univariate $F$ tests showed that this effect was carried by a difference of the mean trend between treated and untreated subjects, $\mathrm{F}(1,36)=7.0, \mathrm{p}<.01$ : Untreated subjects reacted with larger FIRs to spider slides relative to non-spider slides than 
treated subjects. There was no difference between the linear trends, $\mathrm{p}>.20$, indicating a similar habituation pattern for both treated and untreated subjects. When the trend analysis was confined to spider trials, it was found that untreated subjects reacted with larger FIRs to spider trials than treated subjects, $\mathrm{F}(1,36)=6.2, \mathrm{p}<.02$.

Untreated Subjects

- Spider - Weapon - - Flower

Treated Subjects

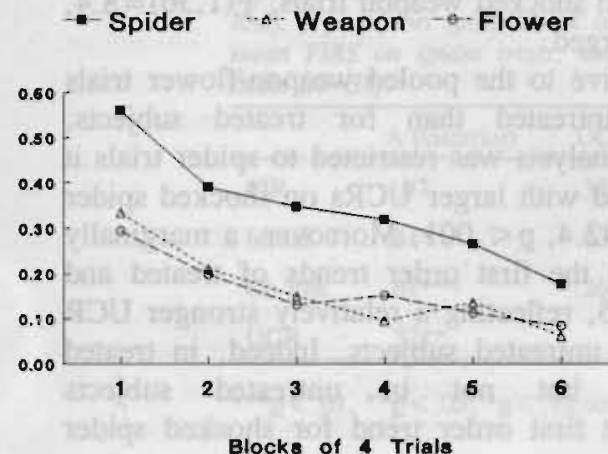

Blocks of 4 Trials

Untreated Subjects

- Spider ... Weapon -a-Flower

कू

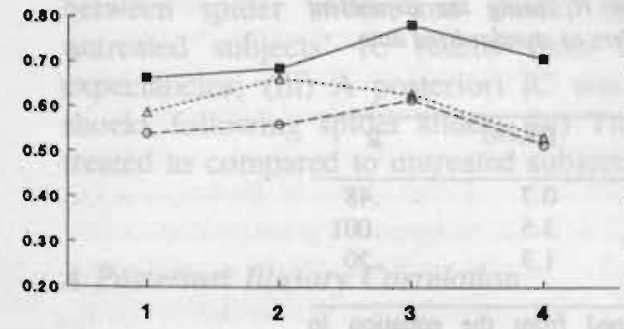

Blocks of 2 Trials
- Spider - Weapon -o-Flower

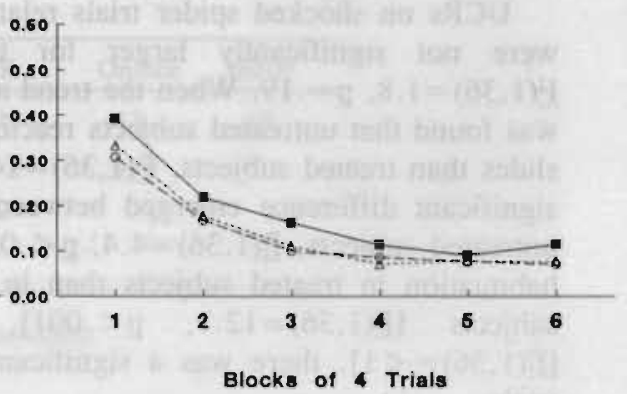

Treated Subjects

- Spider Weapon - Flower

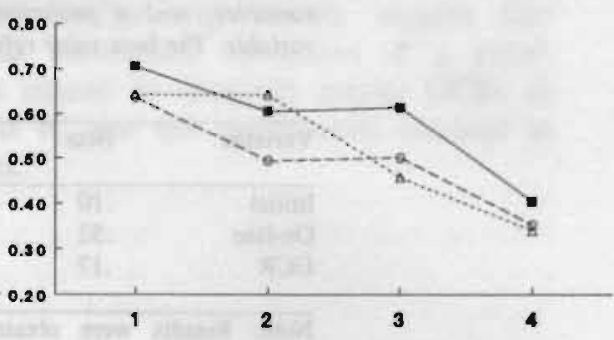

Blocke of 2 Trials

Figure 3.6: The upper panel shows the first interval responses (FIRs) in blocks of 4 trials. The lower panel depicts the unconditioned responses (UCRs) on shocked trials.

The lower panel of Figure 3.6 depicts the UCRs. A MANOVA trend analysis revealed a multivariate effect of slide type for untreated subjects, $\underline{F}(4,33)=7.0, p<.05$. Univariate $\underline{F}$-tests indicated that UCRs on spider trials 
was larger than UCRs on shocked flower and weapon trials, $F(1,36)=12.9$, $\mathrm{p}<.01$, and $\underline{F}(1,36)=23.7, \mathrm{p}<.01$, respectively. In addition, the linear trend of the UCR on spider trials tended to be different from the UCR on shocked flower trials, $\mathbf{E}(1.36)=3.8, \mathrm{p}=.06$. No significant difference emerged between the linear trends of spider and weapon trials $\mathrm{F}(1,36)=1.3, \mathrm{p}=.26$. Thus, no strong evidence for the presence of a differential habituation pattern among slides was obtained.

In treated subjects a significant multivariate effect of slide type was found, $\mathrm{E}(4.33)=3.2, \mathrm{p}<.05$. Univariate $\mathrm{F}$ tests showed that the UCR on shocked spider trials was larger than the UCR on shocked weapon trials, $\underline{F}(1,36)=8.4$, $\mathrm{p}<.01$. No other univariate effects emerged.

UCRs on shocked spider trials relative to the pooled weapon/flower trials were not significantly larger for untreated than for treated subjects, $\mathrm{F}(1,36)=1.8, \mathrm{p}=.19$. When the trend analysis was restricted to spider trials it was found that untreated subjects reacted with larger UCRs on shocked spider slides than treated subjects, $\mathrm{F}(1,36)=142.4, \mathrm{p}<.001$. Moreover, a marginally significant difference emerged between the first order trends of treated and untreated subjects, $F(1,36)=4.4, p<.05$, reflecting a relatively stronger UCR habituation in treated subjects than in untreated subjects. Indeed, in treated subjects $[\mathrm{F}(1,36)=12.1, \mathrm{p}<.001]$, but not in untreated subjects $[\mathrm{F}(1,36)=<1]$, there was a significant first order trend for shocked spider trials.

Table 3.3: Results of the regression analysis with initial spider/shock expectancy (initial), mean on-line spider/shock expeciancy (on-line), and mean UCRs on shocked spider trials (UCR) being the independent variables, and a posteriori IC being the dependent variable. The beta value refers to standardized data.

\begin{tabular}{|c|c|c|c|}
\hline Variable & Beta & $\underline{\lfloor(3,33)}$ & 2 \\
\hline$\overline{\text { Initial }}$ & .10 & 0.7 & .48 \\
\hline On-line & .52 & 3.5 & .001 \\
\hline UCR & .17 & 1.3 & .20 \\
\hline
\end{tabular}

Note: Results were obtained from the equation in which all variables were included $\left(R_{2}=63\right.$, $\underline{E}(3,33)=7.28, \mathrm{q}<.001)$.

To investigate the relative contribution of initial expectancies, on-line processes (as indexed by expectancies and electrodermal responding to shocks following spider slides) in predicting the a posteriori reported spider/shock covariation estimates, a backward regression analysis was performed. In this analysis, only the mean on-line shock expectancy remained in the final equation, indicating that only the on-line expectancies have unique predicting 
properties regarding the a posteriori reported illusory correlation between spiders and shock (see Table 3.3). To obtain more detailed information concerning the interrelationship between initial expectancies, on-line expectancies, skin conductance responses, and a posteriori illusory correlation Pearson's p-m correlations were computed. As can be seen in Table 3.4, initial expectancies, mean on-line expectancies, skin conductance responses, and a posteriori spider/shock estimates were all positively correlated.

Table 3.4: Pearson's p-m correlations between intial spider/shock expectancies (initial), mean on-line spider/shock expectancies (online), a posteriori spider/shock covariation estimates (a posteriori), mean FIRS on spider trials, and mean UCRs on shocked spider trials $(N=38)$.

\begin{tabular}{lllll}
\hline & A posteriori & UCR & On-line & Initial \\
\hline FIR & -.07 & $.35^{* *}$ & -.13 & -.03 \\
Initial & $.31^{*}$ & .03 & $.36^{* *}$ & \\
On-line & $.61^{* * *}$ & $.26^{*}$ & & \\
UCR & $.32^{* *}$ & & & \\
\hline
\end{tabular}

${ }^{* * *} \mathrm{p}<.01,{ }^{* *} \mathrm{p}<.05,{ }^{*} \mathrm{p}<.10$ (one-tailed)

\section{Discussion}

The major results of the present study can be summarized as follows: (i) Untreated spider phobics displayed an a posteriori illusory correlation (IC) between spider slides and shock; (ii) On-line expectancies suggest that untreated subjects' IC results from an incomplete extinction of a priori expectancies; (iii) A posteriori IC was related to relatively strong UCRs to shocks following spider slides; (iv) The IC was not significantly reduced in treated as compared to untreated subjects.

\section{A Posteriori Illusory Correlation}

The present finding that untreated spider phobics a posteriori overestimate the covariation between phobia-relevant stimuli and shock is in line with previous studies (de Jong et al., 1992; Tomarken et al., 1989). It is worth noting that the untreated phobics in the present study overestimated the spider/shock covariation despite the inclusion of both concurrent danger-related stimuli (i.e., aimed weapons) and concurrent danger-related outcomes (i.e., sirens). Thus, the current results add to the evidence (e.g., Tomarken et al., 1989; exp. 2) that the a posteriori reported illusory correlation reflects a selective overassociation of phobia-relevant cues and aversive outcomes rather than a 
general tendency to overassociate salient stimuli.

On basis of the present data it is difficult to draw firm conclusions regarding the treatment effect on covariation bias. On the one hand, the contrast between the spider/shock and the pooled weapon-flower/shock covariation estimates was not found to be significantly different for untreated and treated subjects, indicating that covariation bias was not eliminated by treatment. On the other hand, in untreated subjects the spider/shock association was overestimated in comparison to the weapon/shock, flower/shock, spider/siren, and spider/nothing combinations, whereas in treated subjects none of the relevant comparisons reached significance. In other words, in treated subjects no significant illusory correlation could be demonstrated. Admittedly, the failure to find significant differences between relevant covariation estimates in treated subjects appeared to be due to relatively high non-spider/shock and spider/non-shock estimates rather than to relatively low spider/shock estimates.

This pattern of findings is somewhat at variance with a previous study concerned with treatment effects on covariation bias (de Jong et al., 1992). Although that study followed a similar procedure (i.e., same slides, but tone instead of siren), and although covariation estimates of untreated subjects found in that study were similar to those in this study, de Jong et al. (1992) reported that the bias to overassociate spiders with shock was significantly weaker in treated as compared to untreated women who were spider phobic. One testable explanation for this discrepancy might be that in the current study, on-line judgments affected the a posteriori reported covariation estimates. Pertinent to this possibility is a study of Harkes and Arkness (1983; exp 7). These authors showed that subjects who were asked to keep a "running estimate" during serially presented stimuli reported stronger a posteriori illusory correlations between salient stimuli than subjects who merely had to report a posteriori covariation estimates. Thus, it is possible that in the present study subjects' on-line judgment task contributed to a general inflation of illusory correlations between salient stimuli (e.g., spiders and shocks). It is conceivable that because untreated subjects' spider/shock estimates were already fairly high, this inflation effect would be most prominent in the treated subjects, thereby obscuring the influence of treatment on subjects' a posteriori covariation estimates. Such an explanation would be in accordance with the finding of Tomarken et al. (1989; exp 3) that under certain contextual conditions (i.e., a $50 \%$ base rate of shock rather than a $33 \%$ base rate) low fear subjects display an equally strong bias to overassociate spiders with shock as high fear subjects.

Alternatively, the apparent lack of treatment effect in the present study may indicate that the previously found differences between treated and untreated subjects was not a particularly robust finding. That is, the effects of treatment on covariation bias may be somewhat variable, for instance due to residual fear or (a-specific) sample differences. A similar phenomenon is seen in studies concerned with treatment effects on attentional bias in phobic subjects. Here, some studies have reported a complete disappearance of 
attentional bias in successfully treated phobics (e.g., Watts, McKenna, Sharrock, \& Trezise, 1986), whereas other studies have found in treated subjects an attentional bias that is attenuated, but still clearly present (e.g., Lavy, van den Hout, Arntz, 1992). In passing it should be mentioned that the absence of a reliable treatment effect on illusory correlations by no means indicates that prior fear does not play a role in the generation of illusory correlations. Note, that the remaining fear level of the treated subjects in the present study (as indexed by the SPQ) was still considerably higher than that of the low fear subjects in Tomarken et al.'s study (1989). Further experiments including a control group of non-fearful subjects and both a condition with and a condition without subjects reporting on-line expectancies are necessary to settle more definitely the issue whether or not covariation bias is affected by treatment.

\section{Expectancies and Illusory Correlation}

A major aim of the present study was to investigate how the a posteriori reported ICs are related to initial expectancies. The present results show that already at the start of the experiment, most of the untreated subjects $(78 \%)$ expected an electric shock after the spider slide. However, the same held true for the weapon slide $(72 \%)$. Most importantly, these subjects seemed to correct their initial shock expectancy on weapon trials but not on phobiarelevant trials: The a posteriori weapon/shock estimates were quite correct (32 $\%)$, whereas the spider/shock estimates $(57 \%)$ were inflated. The on-line reported expectancies confirm this pattern. That is, while untreated subjects' expectancy of shock after weapon trials rapidly declined to about $1 / 3$ (the frequency one would expect in subjects making a random choice), they continued to expect shocks after approximately half of the spider slides. Apparently, the spider/shock association is more resistant to disconfirmation than the weapon/shock association. The finding that the majority of subjects expected shock outcome on the first weapon as well as on the first spider trial is in line with a study of McNally and Heatherton (1993) which showed that subjects display a pre-experimental UCS expectancy bias for both phylogenetically (snakes) and ontogenetically fear-relevant (electrical outlets) stimuli. Differential resistance to disconfirmation may explain the failure to find a posteriori ICs between ontogenetically fear-relevant slides and shock (Tomarken, Sutton, \& Mineka, 1994).

Treated subjects significantly less often expected a shock after the first spider slide than untreated subjects ( $37 \%$ vs. $78 \%$ ). However, as for the a posteriori IC, the on-line expectancies did not significantly differ between treated and untreated subjects. Thus, although treated subjects initially did not tend to associate spiders with shock, experiencing the spider/shock combination several times during the experiment seemed to have reinstated the phobiarelevant covariation bias (cf. Davey, 1992; Bouton \& Swartzentruber, 1991). 
Skin Conductance Responses and Illusory Correlation

SCRs paralleled self-report measures. In both groups, FIRs and UCRs were strongest on spider trials during the entire experiment. The FIRs and UCRs were congruent with on-line expectancies and a posteriori reported covariation estimates in that differential responding was most pronounced in untreated subjects. Additionally, in untreated subjects, the habituation of skin conductance responses (UCRs) to shocked spider trials was retarded as compared to treated subjects. This finding indicates that shocks continued to have a greater physiological impact in untreated than in treated subjects, despite the fact that objective shock intensities were comparable for both groups.

The relatively large SCRs on spider trials suggest that the shocked spider trials are not only differentially processed at the cognitive level (i.e., on-line expectancies) but also at the physiological level. The relatively large FIRs during spider slides, which were especially prominent in untreated subjects, indicate that spider slides evoked heightened physiological arousal, probably due to subjects' fear of spiders.

The finding of enhanced UCRs on shocked spider trials replicates earlier results: de Jong and Merckelbach (1991) and de Jong, Merckelbach, and Arntz (1991) also found that following phobia-relevant slides, electrodermal responses to UCSs are enlarged. Thus, it appears that the potentiation of UCRs during phobia-relevant trials is a robust phenomenon. At first sight this phenomenon is difficult to reconcile with two mechanisms that have been extensively described in the literature. First, quantifying SCRs is problematic when a new response is elicited before an immediately preceding response has had time to recover: It is well known that the measurable amplitude of the second response will be smaller given its occurrence following the first response. In fact, the amount of distortion of the second response has been shown to be a function of the size of the first response (Grings \& Schell, 1969). Although in the present experiment slide duration was 8 seconds, it might be that the SCRs to the slides were not completely recovered by the time that shocks were delivered. Since the FIRs were particularly large during spider slides (especially in untreated subjects) this phenomenon would result in relatively small measurable UCRs on shocks preceded by spider slides.

Second, previous studies have clearly demonstrated that UCR magnitude decreases as the probability of the UCS increases (Grings \& Sukoneck, 1971). That is to say, the ability to predict with confidence the occurrence of shock is inversely related to the magnitude of the response elicited by the shock: UCR diminution occurs when neutral stimuli signal shock (Kimmel, 1966) or even when subjects merely think that such stimuli predict shock occurrence (de Jong, Merckelbach \& Arntz, 1990). Given the relatively strong shock expectancies on spider trials one would anticipate relatively small UCRs on shocked spider trials. In contrast to this, the present study found that shocks elicit the strongest UCRs if they are preceded by spider slides. It appears that if stimuli that signal shock possess intrinsic phobic properties (e.g., spider slides for spider phobics) UCR inflation overrides both UCR diminution and the 
distortion of the measurable amplitude due to preceding responses (cf. Merckelbach \& van den Hout, 1991).

The present skin conductance data sustain the idea that shock outcomes after spider slides have a relatively large physiological impact. The large physiological impact of shocked spider trials might be attributed to a match between subjects' affective responses to spiders and to shocks (e.g., Lang, 1985). In its turn, the similarity in emotional responses might promote the phobia-relevant IC. The positive correlation between the FIRs and UCRs on spider trials as well as the positive correlation between UCRs on spider trials and a posteriori reported IC strengthen such interpretation. A recent study of Tomarken et al. (1994) provides further support for this view. The results of that study strongly suggest that the robustness of ICs is related to the similarity of emotional features of stimuli and outcomes (see also Davey, 1993).

\section{Conclusions}

The present findings suggest that the a posteriori reported IC between spiders and shock arises from initial expectancies that survive extinction (untreated subjects) or from expectancies that are reinstated by incidental spider/shock pairings (treated subjects). One of the factors promoting covariation bias might be the potentiated physiological responses on shocked spider trials. These conclusions are sustained by positive correlations between on-line expectancies, a posteriori contingency estimates, and electrodermal responding on shocked spider trials (Table 1). The finding that on-line expectancies, but not initial expectancies, have unique predicting properties for the a posteriori reported IC, underscores the close relationship between on-line processes and the covariation bias phenomenon. 


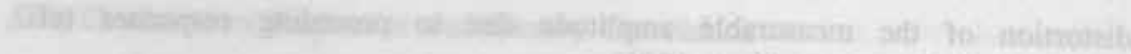

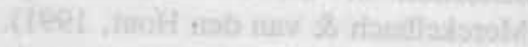

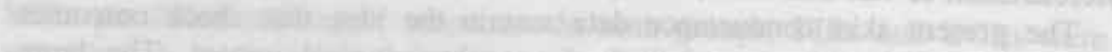

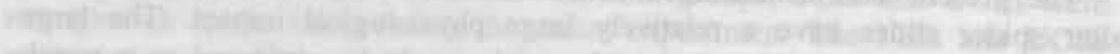

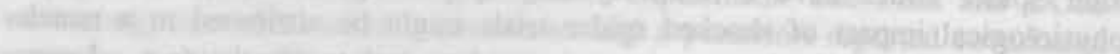

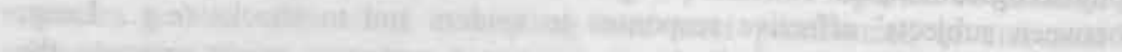
T24.

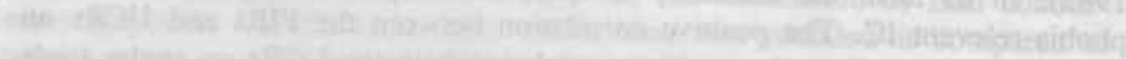
4he

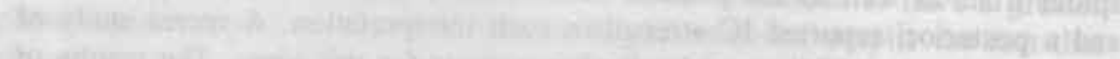

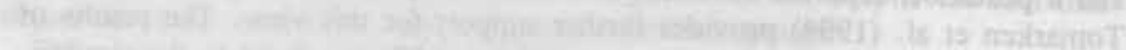
Sin -

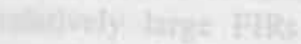

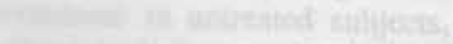

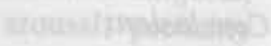

6.7.

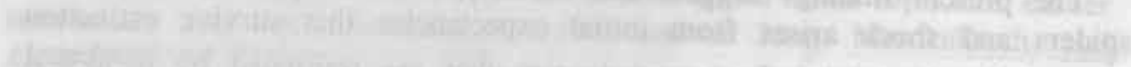

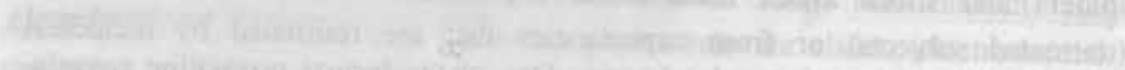

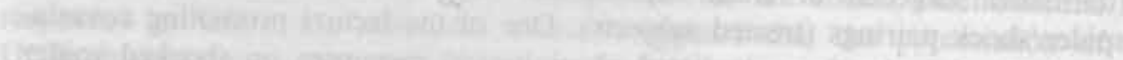

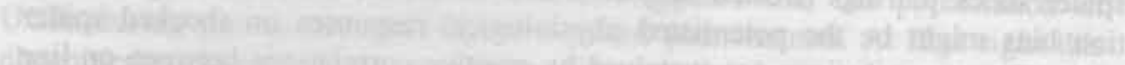

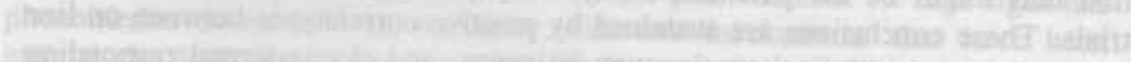

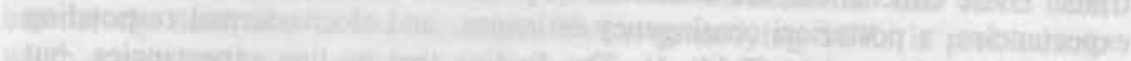

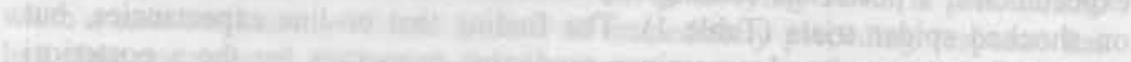

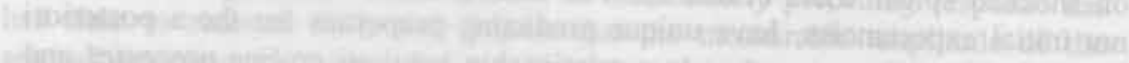

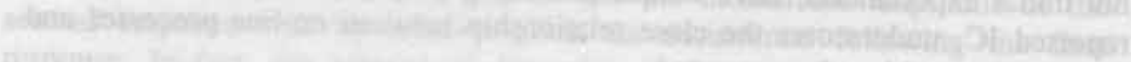

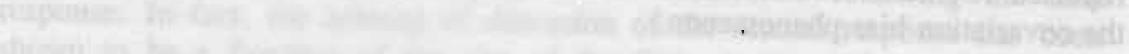

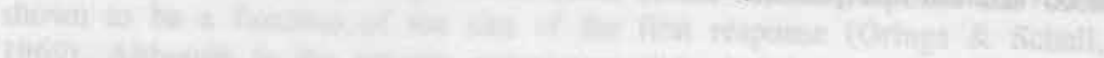

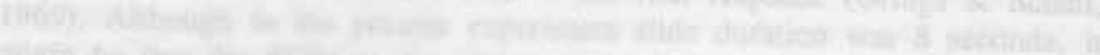

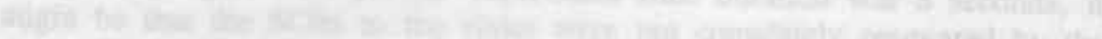


3.d PATHWAYS TO COVARIATION BIAS: HEMISPHERICITY AND IMAGINATION

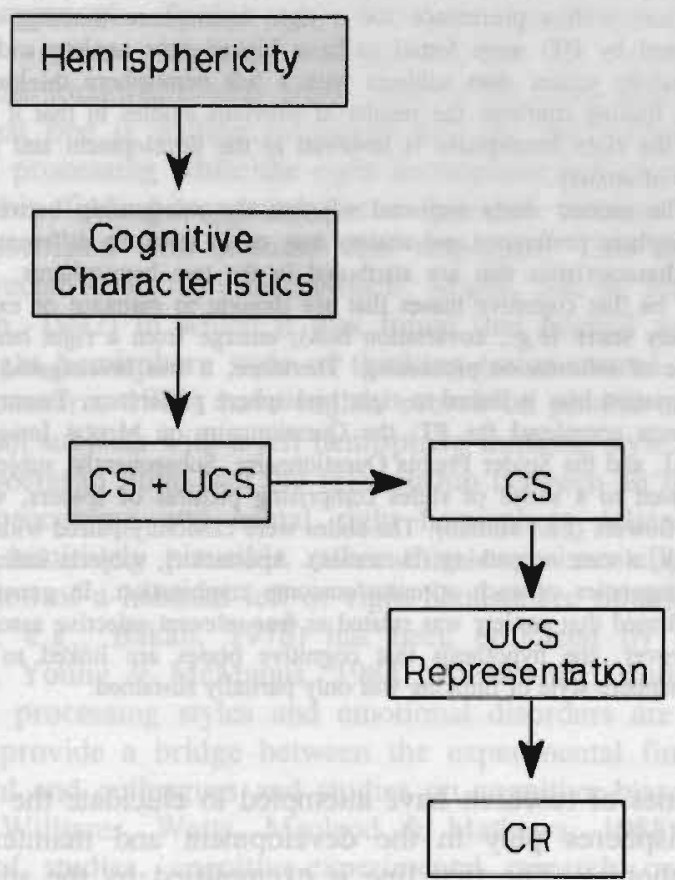

This chapter is based on a paper presented at the 35. Tagung der experimentell arbeitender Psychologen, Osnabrück (1993). A modified version of this chapter is currently under review. 
Hemisphere Preference, Anxiety, and Covariation Bias

Two studies are described. In the first study, normal subjects $(\mathrm{N}=70)$ completed the Preference Test (PT), a paper-and-pencil test presumably measuring preference for a left or a right hemisphere thinking style. Subjects also completed the state version of the Spielberger State-Trait Anxiety Inventory (STAI-state) and the Anxiety Sensitivity Index (ASI). Subjects with a preference for a right hemisphere thinking style (as indexed by PT) were found to have higher state anxiety and anxiety sensitivity scores than subjects with a left hemisphere thinking style. This finding confirms the results of previous studies in that it suggests that the right hemisphere is involved in the development and maintenance of anxiety.

The second study explored whether the relationship between right hemisphere preference and anxiety may result from the different cognitive characteristics that are attributed to the two hemispheres. It might well be that cognitive biases that are thought to maintain or exaggerate anxiety states (e.g., covariation bias), emerge from a right hemisphere mode of information processing. Therefore, it was investigated whether covariation bias is linked to right hemisphere preference. Twenty female subjects completed the PT, the Questionnaire on Mental Imagery, the STAI, and the Spider Phobia Questionnaire. Subsequently, subjects were exposed to a series of slides comprising pictures of spiders, weapons, and flowers (i.e., stimuli). The slides were randomly paired with either a shock, a tone or nothing (outcomes). Aposteriori, subjects indicated the contingencies of each stimulus/outcome combination. In general, data confirmed that anxiety was related to fear-relevant selective associations. However, the hypothesis that cognitive biases are linked to a right hemisphere style of thinking was only partially sustained.

Two recent lines of research have attempted to elucidate the role that the two cerebral hemispheres play in the development and maintenance of human anxiety and phobias. The first line is exemplified by the studies of Hugdahl $(1987,1989)$ in which fairly direct experimental manipulations (e.g., tachitoscopic procedures) were employed to assess the differential involvement of the two hemispheres in fundamental processes such as orienting and classical conditioning. These studies have shown, for example, that fear-relevant stimuli (e.g., pictures of snakes) elicit a cardiac defence reaction when they are flashed to the right hemisphere, but not when they are flashed to the left hemisphere (Hugdahl, Franzon, Andersson \& Walldebo, 1983; see also Dimond \& Farrington, 1977). Another interesting finding was that slides of angry faces that are flashed to the right hemisphere and then paired with aversive shock, evoke a conditioned skin conductance during a next phase in which these slides are foveally presented. However, such a conditioned response does not occur when slides have been presented to the left hemisphere and then paired with shock (Hugdahl \& Johnsen, 1991). By and large, 
the results of Hugdahl and associates suggest that the right hemisphere of normal subjects is more sensitive to fear-relevant stimuli and is more likely to acquire a conditioned fear response than the left hemisphere.

The second line of research relies on indirect measures of hemisphere involvement in anxiety. For example, Tucker and Newman (1981; see also Shearer \& Tucker, 1981) confronted normal subjects with emotional slides, among them fear-relevant pictures (e.g., disfigured bodies, snakes etc.). One group of subjects was instructed to process these slides in a analytic/verbal way, whereas the other group of subjects were instructed to employ an imaginal/global approach. Tucker and Newman found that the emotional material elicited greater peripheral vasoconstriction (as measured by skin temperature) in the imaginal/global group than in the analytical/verbal group. To the extent that one is willing to accept that the left hemisphere has an analytic mode of processing while the right hemisphere has an imaginal mode of processing, this finding suggests that a habitual reliance on the right hemisphere is associated with stronger fear responses. This suggestion was underlined in a recent study by Merckelbach, Muris, and de Jong (1991; see also Merckelbach, 1992) in which it was found that normal subjects with a predominantly right hemisphere style of thinking (as measured by the Preference Test; Zenhausern, 1981) have higher scores on phobia and depression questionnaires than subjects with a left hemisphere thinking style.

It should be borne in mind that the relationship between an imaginal mode of information processing and actual right hemisphere reliance is largely inferential (i.e., intuitively plausible rather than experimentally proven). In addition, the notion of a habitual left or right hemisphere thinking style (i.e., "hemisphericity"; e.g., Bakan, 1978) has been criticized by some authors (e.g., Beaumont, Young \& McManus, 1984). Meanwhile, studies concerned with information processing styles and emotional disorders are important in that they might provide a bridge between the experimental findings of, for example, Hugdahl and colleagues and studies on cognitive biases in neurotic disorders (e.g., Williams, Watts, Macleod \& Mathews, 1988). As for the latter category of studies, cognitive-experimental research on anxiety has found, among other things, that phobic patients tend to overestimate the contingency between fear-relevant stimuli (e.g., slides of spiders) and aversive events (e.g., electric shock), even when there is no systematic correlation between stimuli and aversive events (e.g., Mineka \& Tomarken, 1989; de Jong, Merckelbach, Arntz, \& Nijman, 1992). In other words, phobics tend to perceive an illusory correlation between fear-relevant stimuli and aversive experiences. It may well be that this tendency acts to maintain the phobic complaints (see for an analysis that comes close to this suggestion McNally \& Foa, 1987). Cognitive biases such as the tendency to perceive illusory correlations might be related to certain information processing characteristics (a non-analytic, holistic approach; overreliance on imagery etc.) which, in turn, are linked to right hemisphere functioning. In more general terms, then, it might be worthwhile to explore whether cognitive biases in fearful subjects can be pinpointed to neuropsychological mechanisms (e.g., Power, 1991). 


\section{Study I}

Given the potential clinical relevance of research concerned with information processing styles and anxiety, the first study attempted to replicate the previously reported association between right hemisphere thinking style and anxiety scores that was found in normals (Merckelbach et al., 1991). In that study, anxiety was measured by means of a self-report questionnaire (i.e., the Fear Questionnaire; Marks \& Mathews, 1979) that asks for the presence of a limited set of phobias. In contrast, the present study examined the relationship between hemisphere thinking styles and a broader index of anxiety, namely the state anxiety version of the Spielberger State-Trait Anxiety Inventory (STAI state; Spielberger, Gorsuch \& Lushene, 1970). Additionally, subjects completed the Anxiety Sensitivity Index (ASI; Reiss, Peterson, Gursky \& McNally, 1986), a self-report instrument that measures fear of bodily arousal sensations.

\section{Method}

\section{Subjects}

Subjects were 70 undergraduate students $(28 \mathrm{men})$. Their mean age was 22.2 years (range: $18-39$ years).

\section{Procedure and Assessment}

Subjects were invited to complete the following questionnaires: the Preference Test (PT; Zenhausern, 1981), the state anxiety version of the STAI (Spielberger et al., 1970), and the ASI (Reiss et al., 1986). Subjects completed the questionnaires in their regular class room.

The PT is a 20 item paper and pencil test and measures "style of thinking": 10 items address what can be termed a "right hemisphere mode of thinking" (right hemisphere items, e.g., "I have a good sense of direction") and 10 items address a "left hemisphere mode of thinking" (left hemisphere items, e.g., "I find it easy to think of synonyms for words"). It is worthy of note that the PT does not contain "emotional" items. Thus, the extent to which the PT items are susceptible to mood-congruent effects seems limited. Subjects use 10-point scales (ranging from $0=$ "not al all"/"never" to $10=$ "very much"/"always") to indicate the degree to which the items apply to them. To obtain an index of hemisphere reliance or preference, the mean score on the right hemisphere items is subtracted from the mean score on the left hemisphere items. Thus, a positive difference score reflects a stronger preference for a left hemisphere thinking style (i.e., an analytic, verbal approach). In contrast, a negative difference score reflects a stronger reliance on a right hemisphere thinking style (i.e., a holistic, non-verbal approach). 
While the connection between PT and hemisphere (over) activity is inferential, there are some indications that the PT correlates with other (non-obtrusive) measures of hemisphere reliance (e.g., conjugate lateral eye movements; de Jong, Merckelbach \& Muris, 1990; see also Zenhausern, Notaro, Grosso \& Schiano, 1981). Furthermore, reading efficiency has been found to correlate with PT scores, with poor readers having a negative PT difference score (i.e., a right hemisphere thinking style; Oexle \& Zenhausern, 1981).

The state anxiety version of the STAI (Spielberger et al., 1970) is a widely used questionnaire with good psychometric properties. It contains twenty items (e.g., "I feel scared") that are rated on a 4-point scale (ranging from "not at all" to "very much"). Scores are summed to obtain a total state anxiety score (maximum $=80$ ).

The ASI (Reiss et al., 1986) is a 16-item self-report scale. The ASI items focus on fear of anxiety symptoms (e.g., "it scares me when my heart beats rapidly"). The items are rated on a 5-point scale ranging from 0 ("very little") to 4 ("very much"). Scores are summed to obtain a total ASI score (maximum $=64)$. The ASI has adequate test-retest stability and has been validated for non-clinical populations (e.g., Donnell \& McNally, 1990).

\section{Results}

Pearson p-m correlations between PT, STAI, and ASI are presented in Table 3.5. As can be seen, PT correlates negatively with STAI: the stronger a person's reliance on a left hemisphere thinking style, the lower his or her state anxiety scores. The association between PT and ASI was also negative, but did not reach the conventional significance level. As was expected, the two anxiety measures correlate positively.

Table 3.5: Pearson productmoment correlations between PT, STAI-state, and ASI.

\begin{tabular}{lll}
\hline & STAI-state & ASI \\
\hline PT & $-.29^{*}$ & $-.13^{* *}$ \\
ASI & $.45^{*}$ \\
\hline$* \mathrm{p}<0.01, \mathrm{n}=70$, (one-tailed) \\
$* \mathrm{p}<0.15, \mathrm{n}=70$ (one-tailed)
\end{tabular}

On the basis of the distribution of the PT scores, three groups of subjects were formed; subjects scoring in the upper $20 \%$ of the distribution $(n=14$; subjects with a relative strong left hemisphere preference), subjects scoring in the lower $20 \%$ of the distribution ( $n=14$; subjects with a relative strong right hemisphere preference), and the remaining subjects $(n=42$; subjects with a 
mixed hemisphere preference). The mean state anxiety and ASI scores of the three groups are shown in Table 3.6. State anxiety and ASI scores of the three groups were subjected to one-way analyses of variance (ANOVAs). The ANOVA performed on the ASI scores did not yield a significant effect: $F(2,69)=1.69, p=0.19$. Yet, when the mean ASI scores of the left hemisphere preference and the right hemisphere preference group were compared with a $\mathrm{t}$-test, a significant difference emerged $[\mathrm{t}(26)=1.80$, $\mathrm{p}<0.05$ one-tailed], with the latter group having higher ASI scores. The ANOVA of the state anxiety scores yielded a significant effect: $F(2,60)=$ $3.78, \mathrm{p}<0.05$. The right hemisphere preference group had significantly higher state anxiety scores than the left hemisphere preference group $[\mathrm{t}(26)=2.56$, $\mathrm{p}<0.01$, one-tailed].

Table 3.6: Mean STAI-state and ASI scores of the right hemisphere thinking style group, the "mixed" group, and the left hemisphere thinking style group. Standard deviations are given between parentheses.

STAI-state

38.6

Right Hemisphere Style $(n=14)$

Mixed Style $(n=42)$

Left Hemisphere Style $(n=14)$
34.08
ASI

\section{Discussion}

The results presented above can be summarized as follows. Firstly, although not of a dramatic magnitude, correlations were found between hemisphere thinking style and anxiety scores. Subjects with a relatively strong reliance on a right hemisphere thinking style were found to have higher state anxiety and anxiety sensitivity scores than subjects with a relatively strong reliance on a left hemisphere thinking style. Taken together, these results confirm the findings of an earlier study (Merckelbach et al., 1991).

There are at least two theories that may account for the connection between hemisphere thinking style and anxiety. The first theory assumes that there is an inborn affective asymmetry between the hemispheres. Although there is a lack of consensus about the details of this affective lateralization, many authors believe that the right hemisphere sustains negative emotions, whereas the left hemisphere sustains positive emotions (Davidson \& Fox, 1984; Weber \& Sackheim, 1984). Activation of one rather than the other hemisphere, for example, through certain cognitive activities would evoke a 
direct emotional effect. There is some evidence to support this notion (see for a review, Silberman \& Weingartner, 1986). For example, Drake (1987) instructed normal subjects to shift their eyes either to the left or to the right, thereby inducing contralateral cerebral activation. Orienting towards the right (i.e., left hemisphere activation) was followed by positive evaluations of pictorial stimuli. In contrast, orienting towards the left (i.e., right hemisphere activation) was followed by negative evaluations of these stimuli (see also Merckelbach \& van Oppen, 1989).

The second theory stresses the different cognitive characteristics of the two hemispheres. The rational and verbal strategy of the left hemisphere would inhibit emotional responses, while the nonverbal and imaginal ideation of the right hemisphere would exaggerate emotional responding (e.g., Tucker \& Newman, 1981). Thus, emotions would be modulated by lateralized cognitive characteristics rather than produced by inborn affective tendencies of the two hemispheres. If this cognitive point of view is correct, one would predict that cognitive biases that promote anxiety responses (i.e., the illusory correlation phenomenon; see below) are more often found in persons who rely on right hemisphere strategies than in persons who rely on left hemisphere strategies.

\section{Study II}

The second study tentatively explored whether cognitive biases such as illusory correlation are, indeed, related to an imaginal mode of information processing. In a recent study employing an "illusory correlation" paradigm, it was demonstrated that the strength of the bias to overassociate fear-relevant stimuli with shock is correlated with the severity of phobic complaints (de Jong, Merckelbach, Arntz \& Nijman, 1992). Employing the same illusory correlation paradigm, the present study investigated if and to what extent covariation bias is correlated with a right hemisphere mode of thinking. Houtz and Frankel (1988) provided empirical support for the hypothesis that subjects with a right hemispheric preference are less successful in problem-solving tasks than subjects with a left hemisphere preference. In a similar vein, it might well be that an imaginative approach will lead to less accurate covariation detection in an "illusory correlation" paradigm than a more analytic approach. Inaccurate covariation detection, in turn, may lead to (or confirm) an unrealistic representation of cue-outcome relationships (e.g., between phobic stimuli and aversive consequences). From this perspective, covariation bias might be one of the mediating factors between right hemisphere preference and anxiety.

A recent study by Hugdahl and Johnsen (1991) provides further support for this line of reasoning. In that study, subjects were presented with fearrelevant stimuli flashed either to the right (RVF; i.e., left hemisphere) or to the left visual field (LVF; i.e., right hemisphere). During the acquisition phase, the lateralized stimuli were paired with shock outcome. Only in case stimuli had been presented in the LVF, subsequent stimulus presentations (in 
the visual centre) elicited conditioned electrodermal responses. This suggests that the right hemisphere is more susceptible to associate fear-relevant stimuli with aversive outcomes.

In the present study, both the Zenhausern Prefence test (PT) and the Questionnaire on Mental Imagery (QMI) were used as indices of hemisphere preference. It was anticipated that subjects with negative PT scores (indicating non/verbal/holistic approach) and subjects with low QMI scores (indicating strong imagery) would show a stronger covariation bias than analytic subjects and/or subjects with only weak imaginative power.

\section{Method}

\section{Subjects}

Subjects were 20 female undergraduate students. The mean age was 21 (range: 18-24 years). Subjects were paid for their participation in this experiment. Due to apparatus failure, covariation data of one subject was not available, leaving 19 subjects in the final sample.

\section{Assessment}

Before the experiment proper, subjects were asked to complete several questionnaires: As in study 1, subjects completed the PT and the STAI-state. Additionally, subjects were invited to complete the Spider Phobia Questionnaire (SPQ; Klorman, Weerts, Hastings, Melamed \& Lang, 1974), and the Questionnaire on Mental Imagery (QMI; Sheenan, 1967).

The SPQ is a 31-item self-report instrument that measures fear of spiders and has reasonable psychometric properties (Fredrikson, 1983).

The QMI is a widely used index of imagery ability (e.g., Cook, Melamed, Cuthbert, McNeil \& Lang, 1988; Merckelbach, de Jong \& Arntz, 1991). It contains 35 items which pertain to images in specific modalities (e.g., in the visual modality: 'seeing a sunset'; in the auditory modality: 'hearing steam escape from a boiling kettle'; etc.). Subjects are asked to rate the vividness of these images on a 7-point scale, with 1 indicating 'very vivid' and 7 indicating 'not at all vivid'. Thus, the total QMI score varies between 35 (very good imagery ability) and 245 (very poor imagery ability).

\section{Stimulus Material and Apparatus}

In the experiment proper, three categories of slides were used: 4 different slides depicting spiders (phobogenic stimuli), 4 different slides depicting aimed weapons (ontogenetically fear-relevant stimuli), and 4 different slides depicting flowers (neutral stimuli). The slides were projected on a white 
screen $(80 \times 120 \mathrm{~cm}), 3 \mathrm{~m}$ in front of the subject. A Kodak Carousel was used for stimulus presentation. Following each stimulus, three types of outcomes could occur: a 1-s shock, a 1-s tone, or nothing at all. Electrical shocks (dc) were delivered from a specially designed shock generator and administered to the subjects' lateral side of the upper right arm through two electrodes $(8 \mathrm{~mm}$ diameter $\mathrm{Ag}-\mathrm{AgCl}$ ). Tones were delivered by a tone generator $(60 \mathrm{~Hz}, 50 \mathrm{~dB})$ connected to a loudspeaker inside the experimental (sound attenuated) room. Stimulus presentation, delivery of shocks and tones, and intertrial intervals were controlled by a PDP Minc II laboratory computer.

\section{Procedure}

At the completion of the questionnaires, subjects were introduced to the laboratory. During the experiment, subjects sat in a chair in a soud-attenuated room. A one-way screen seperated the experimental and the apparatus room. Subjects were explicitly informed that is was their task to determine the relationship between categories of slides and outcomes. Following this, electrodes were attached. Then, shock intensity level was determined using a shock work-up procedure. Stepwise, electrical current was increased until the subject indicated that the shock was "uncomfortable but not painful". After it was established that the task was clear to the subjects, they were left alone and the lights were dimmed.

Next, subjects were exposed to 72 slides each of 8 s duration. Three different categories were used (see above). One of three outcomes occurred at slide offset: a shock (aversive outcome), a tone, or nothing at all (neutral outcomes). All slide-outcome combinations occurred equally frequently, thus the conditional probability of each slide type given the prior occurrence of each outcome was $1 / 3$. Slides were presented in a quasi random order with the restriction that a similar slide-outcome combination never appeared on two successive trials. Across subjects, three different sequences of slides were used in order to control for possible primacy and latency effects. Therefore, each of the sequences started with an other slide type.

After the experiment, subjects completed the Covariation Questionnaire (CQ). The CQ asked subjects to estimate the percentage of occurrence of each outcome given the prior occurrence of each slide type. A sample item would be as follows: "Given that you saw a weapon slide, on which percentage of those trials weapons were followed by shock?" For all estimates, $100 \mathrm{~mm}$ visual analog scales (VASs) were used ranging from $0 \%$ (i.e., never) to $100 \%$ (i.e., always).

\section{Data Reduction and Analysis}

In line with the earlier covariation studies of Tomarken, Mineka, and Cook (1989), covariation estimates were subjected to a set of a-priori t-tests in order 
to evaluate whether the spider/shock covariation estimates differed from the weapon/shock, flower/shock, spider/tone, and spider/nothing covariation estimates. The standard deviation (SD) of the reported estimates (within subjects) was used as an additional index of the subjects' ability to detect covariations. Note that all covariations between slides and outcomes were equal. Consequently, the smaller the standard deviation, the more accurate the subjects' covariation estimates. Conversely, the higher the standard deviations, the greater the discrepancies between the various stimulus-outcome estimates, the poorer the judgments. Both measures of covariation bias were used in the correlational analysis: Standard deviations (SD) over all covariation estimates (within subjects) and the covariation estimates of the spider/shock associations (IC). Pearson p-m correlations were computed between hemisphericity (QMI, PT), anxiety (SPQ, STAI), and subjects' ability to detect covariations (SD, IC).

\section{Results and Discussion}

Questionnaires. Mean SPQ score was 9.9 (range 2 - 28). The distribution was comparable to the distribution reported by Fredrikson (1983) for his "normal" student sample. Scores on the QMI, PT and STAI-state are depicted in Table 3.7.

Table 3.7: Mean scores and range of the Preference Test (PT), Questionnaire on Mental Imagery (QMI), Spider Phobia Questionnaire (SPQ), and the STAI-state.

\begin{tabular}{lllll}
\hline & PT & QMI & SPQ & STAI-state \\
\hline mean & 0.96 & 79.5 & 9.9 & 34.1 \\
(range) & $(-27.6-45.7)$ & $(47-104)$ & $(2-28)$ & $(23-50)$ \\
\hline
\end{tabular}

Covariation Estimates. Across all subjects, the spider/shock estimates (IC) differed significantly from the weapon/shock estimates, $\mathrm{t}(18)=3.17, \mathrm{p}=.005$, but not from the flower/shock estimates, $\mathrm{t}(18)<1$. In addition, the difference between the spider/shock estimates and the spider/tone estimates was marginally significant, $\mathrm{t}(18)=2.48, \mathrm{p}=.023$. Finally, the spider/shock estimates tended to be higher than the spider/nothing estimates, $t(18)=2.26, \mathrm{p}=.036$. Thus, in general, subjects showed a weak bias to overassociate the spider/shock combination.

Hemisphere Preference, Covariation Bias, and Phobic Fear

Correlations between indices of covariation bias, anxiety, and hemisphere 
preference are shown in Table 3.8. As was predicted, there was a significant correlation of the QMI score with subjects' accuracy of covariation detection, suggesting that inaccurate covariation detection is related to a right hemisphere mode of information processing. However, the correlations between the other 'hemisphericity' index (PT) and both measures of covariation bias (SD and IC) did not attain significance. Thus, at best, the present results only partially sustain the hypothesis that cognitive biases are linked to a right hemisphere style of thinking.

Table 3.8. Correlations between indices of anxiety (SPQ. STAl), hemisphericity (PT, QMI) and covariation bias (IC=spider/shock estimates; $S D=$ accuracy).

\begin{tabular}{llllll}
\hline & IC & SD & STAI & SPQ & PT \\
\hline QMI & -.05 & $-.50^{* *}$ & .00 & -.09 & .10 \\
PT & .17 & .12 & $-.26^{*}$ & -.05 & -- \\
SPQ & $.42^{* *}$ & .04 & $.34^{*}$ & $\ldots$ & $\ldots-$ \\
STAI & .20 & $.31^{*}$ & $\ldots$ & $\ldots$ & $\ldots-$ \\
\hline
\end{tabular}

$*_{\mathrm{D}}<.15, \mathrm{n}=19$ (one-tailed)

${ }^{* *} \mathrm{Q}<.05, \mathrm{n}=19$ (one-tailed)

In line with the hypothesis that inaccurate covariation detection may be related to anxiety, the index of anxiety (STAI) was positively correlated with subjects' inaccuracy in detecting covariations. However, these correlations did not reach the conventional level of significance. In addition, there was a significant positive correlation between the index of phobic fear of spiders (SPQ) and the spider/shock covariation estimates (IC). This result is in line with earlier findings that high but not low fear subjects show a covariation bias (e.g., Tomarken et al., 1989; de Jong et al. . 1992). Yet, the present findings provide no support for the suggestion that this relationship between covariaton bias and phobic fear c.q. anxiety is mediated by hemisphere preference. Finally, no significant correlation could be found between the PT and QMI scores. On the one hand this result is difficult to reconcile with the widely hold assumption that imagery is a function of the right hemisphere (e.g., Springer \& Deutsch, 1981). On the other hand, it may be taken as support for the position of Ehrlichman and Barret (1983) who argue that there is insufficient empirical basis for considering imagery a right hemisphere function. It might well be, as Farah $(1984$, p. 268) stated, that "the right hemisphere hypothesis does not actually apply to image generation per se, but rather to various forms of so-called 'spatial ability' and higher visual perceptual processing". In other words, visual vs nonvisual thinking style might be closer related to hemisphericity than imagery ability per se (see also Zenhausern, 1978). 


\section{General Discussion}

The present study was performed to replicate and extent the earlier finding that a relatively strong reliance on a right hemisphere thinking style is related to higher levels of anxiety (Merckelbach et al., 1991). In addition, it was explored whether this relationship between hemisphere preference and anxiety is mediated by the different cognitive characteristics that are ascribed to the left and right hemispheres.

Pertinent to the first aim of this study, the present results corroborate the earlier finding that right hemisphere preference is a vulnerability factor for anxiety (Merckelbach et al., 1991). Hemisphericity was found to be related to state anxiety (STAI-state) as well as fear of bodily sensations (ASI).

At least two mechanisms can be put forward to explain this link between right hemisphere dominance and anxiety. First, a study by Davidson and Fox (1989) provides evidence for the suggestion that right frontal activation marks a vulnerability factor to experience negative emotions. That is, the threshold for experiencing and expressing negative affect due to a certain stressor is lowered in subjects showing relatively strong right frontal hemisphere activation. Thus, it might be inferred that subjects predominantly relying on a right hemispere mode of information processing are characterized by relatively strong right frontal activation. In its turn, this strong right activation might increase subjects' susceptibility to negative emotions like anxiety (see e.g., Silberman \& Weingartner, 1986).

The second mechanism linking hemisphericity and anxiety might be constituted by the different cognitive characteristics of both hemispheres. For example, Tucker and Newman (1981) demonstrated that global and imaginal thinking exaggerates emotional experiences, whereas verbal and analytic ideation seems to effectively inhibit emotional responses. From this it may be inferred, that subjects with a right hemispheric style of information processing might be prone to anxiety due to the cognitive characteristics of the right hemisphere (i.e., its capacity for global and conceptual integration of sensory with visceral cues). That is, subjects typically relying on right hemisphere strategies would be more prone to cognitive biases (e.g., covariation bias) that are known to maintain or exaggerate fear responses (i.e., covariation bias) than subjects preferring a left hemisphere style of thinking. This prediction was tested in experiment 2.

The current findings are in line with earlier results (e.g., de Jong et al., 1992), in that they show that high fear subjects are likely to overestimate the association between fear-relevant stimuli and aversive outcomes. Similarly, high state anxious subjects appeared to be less accurate than low anxious subjects in detecting covariations in an illusory correlation paradigm. However, the prediction that subjects with a right hemisphere style of thinking are prone to cognitive biases such as covariation bias was, at best, only partially confirmed. Only the relationship between imagery ability and subjects' overall inaccuracy in covariation detection (SD) pointed in that direction. Yet, right hemisphere thinking style (indexed either as imaginative power, or as visual 
thinking style) was not correlated with a specific bias to associate fear-relevant stimuli with shock (IC).

Of course, the present failure to find a significant relationship between hemisphericity and specific covariation bias does not imply that there is no such a relationship. Selecting subjects with more extreme preference for a right or a left hemispheric mode of information processing might have yielded quite different results. In addition, rather indirect indices of hemisphericity were used in the present experiment. It might well be the case that, given the relatively small sample size, these indices were lacking of sufficient sensitivity. Meanwhile, the fact that imagery ability and covariation detection accuracy were found to be associated suggests that it might be worthwhile to evaluate the connection between hemisphericity and cognitive bias with a more straightforward index of hemisphere functioning (e.g., baseline EEG; Davidson \& Fox, 1989).

To summarize, the present study corroborates the hypothesis that right hemisphere preference is positively related to high anxiety levels. The hypothesis that cognitive characteristics linked to each hemisphere mediate the relationship between hemisphere thinking style and anxiety was, at best, only partially sustained. 


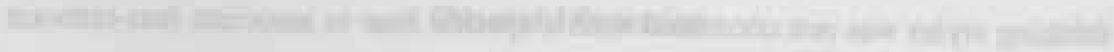

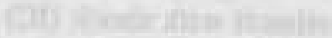

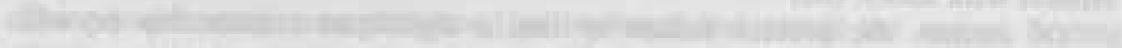

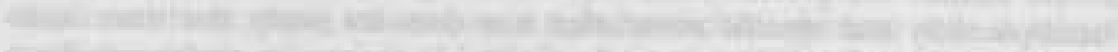

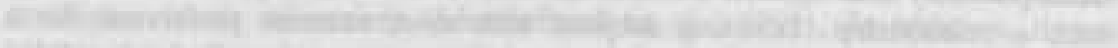

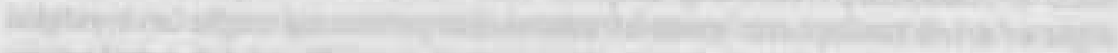

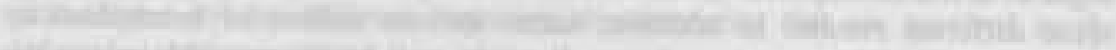

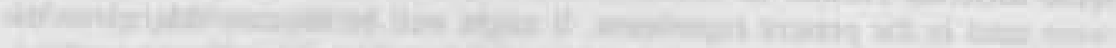

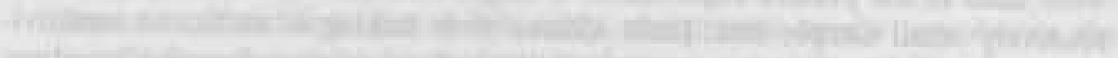

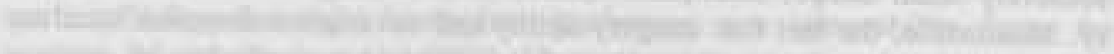
an-

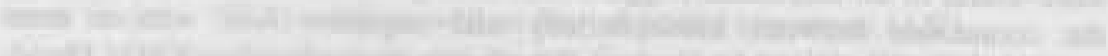

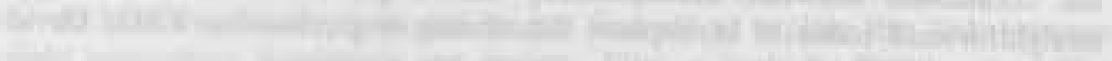

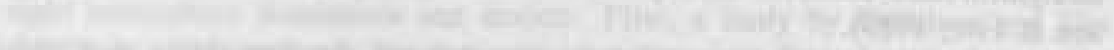
(1)

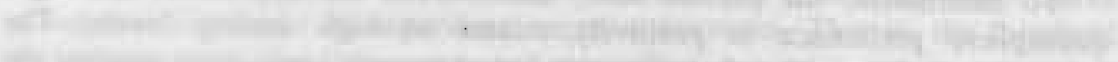

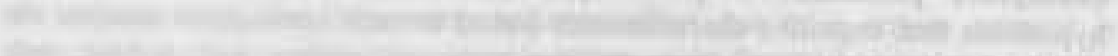

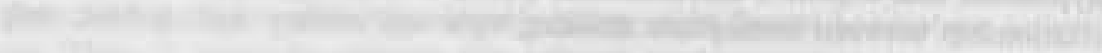

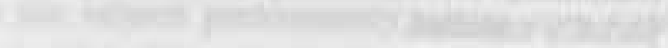
15 


\section{4.a COVARIATION BIAS: CAUSE OR CONSEQUENCE?}

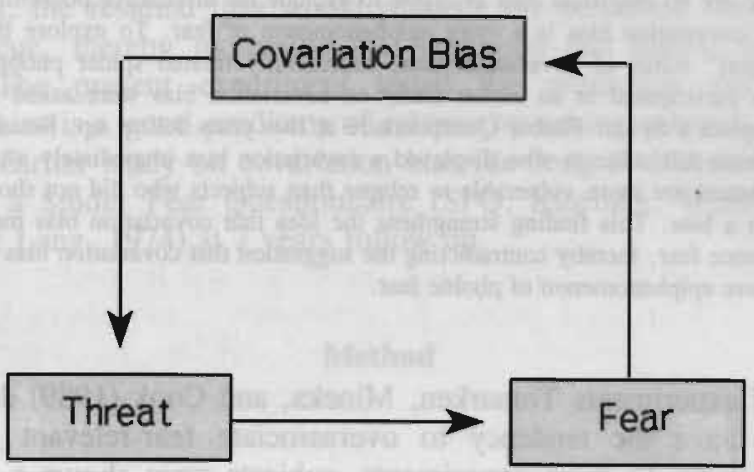

This chapter is based on a paper presented at the 23rd Congress of Behaviour and Cognitive Therapies (London, September 1993). A modified version of this chapter is published as: de Jong. P.J., van den Hout, M.A.. \& Merckelbach, H. (1994). Covariation bias and the return of fear. Behaviour Research and Therapy, in press.

Acknowledgements: We thank Annie Raven for her assistance during the data acquisition. 


\title{
Covariation Bias and the Return of Fear
}

\begin{abstract}
Several studies have indicated that phobic fear is accompanied by a covariation bias, i.e., that phobic subjects tend to overassociate fear relevant stimuli and aversive outcomes. Such a covariation bias seems to be a fairly direct and powerful way to confirm danger expectations and enhance fear. Therefore, it has been suggested that covariation bias is an important factor in the maintenance of phobic fear. However, thus far there are no empirical data available to exclude the alternative possibility that covariation bias is a mere epiphenomenon of fear. To explore the "causal" status of covariation bias, successfully treated spider phobics who participated in an earlier study on covariation bias were asked to complete a Spider Phobia Questionnaire at two years follow up. Results indicate that subjects who displayed a covariation bias immediately after treatment are more vulnerable to relapse than subjects who did not show such a bias. This finding strengthens the idea that covariation bias may enhance fear, thereby contradicting the suggestion that covariation bias is a mere epiphenomenon of phobic fear.
\end{abstract}

In a series of experiments Tomarken, Mineka, and Cook (1989) demonstrated that subjects have the tendency to overassociate fear-relevant stimuli and aversive outcomes. In these experiments, subjects were shown a large series of fear-relevant (snakes or spiders) and fear-irrelevant (flowers and mushrooms) slides. Immediately at slide offset one of three outcomes occurred: an aversive shock, a tone, or nothing. All slide/outcome combinations were presented equally often. In other words, the conditional probability of a certain outcome given a certain slide was always $1 / 3$. After the experiment proper subjects were asked to rate how often each slide type was followed by each outcome. Although all slide/outcome combinations occurred equally often, subjects typically overestimated the covariation between fear-relevant slides and aversive shocks. This so-called "covariation bias" appeared to be particularly strong in high fear subjects. Further research of de Jong, Merckelbach, Arntz, and Nijman (1992) with clinical rather than analog spider phobics confirmed that phobic fear is, indeed, an important determinant of such a covariation bias. In that study it was found that untreated women who were spider phobic strongly overestimated the covariation between phobiarelevant slides and shock whereas successfully treated phobics did not.

It has been suggested that a bias to overestimate the contingency between phobic stimuli and aversive events logically serves to confirm and/or to enhance fear (e.g., Mineka \& Sutton, 1992; de Jong \& Merckelbach, 1993). That is, fear may induce covariation bias; in turn, covariation bias sustains/enhances perceived threat; perceived threat maintains/intensifies fear, etc. Thus, covariation bias would be an important factor in the maintenance and enhancement of phobic fear. However, despite its face validity no empirical data are available to confirm or disconfirm the presence of a reciprocal relationship between covariation bias and phobic fear. Although 
both the finding that especially high fear subjects show a covariation bias (Tomarken et al., 1989) and the finding that this bias can be reduced as a result of treatment (de Jong et al., 1992; de Jong \& Merckelbach, 1993) fit with the presumed relationship, neither of these findings excludes the alternative possibility that covariation bias is a mere epiphenomenon of phobic fear.

Yet, a critical implication of the proposed reciprocal relationship between phobic fear and covariation bias would be that successfully treated phobics who nevertheless show a post-treatment covariation bias, are more vulnerable to relapse than subjects who do not display such a post-treatment bias. That is, the residual bias would enhance the perceived threatfullness of the phobic cue, thereby increasing the likelihood that phobic fear becomes reinstated. The present experiment tested the prediction that a residual covariation bias is a good predictor of relapse by asking subjects who participated in an earlier study on covariation bias (de Jong \& Merckelbach, 1993) to complete a Spider Fear Questionnaire (SPQ; Klorman, Weerts, Hastings, Melamed, \& Lang, 1974) at 2 years follow up.

\section{Method}

\section{Subjects}

Subjects were 19 treated spider phobics who participated in an earlier study on covariation bias and phobic fear (de Jong \& Merckelbach, 1993). All subjects were women. In that study, subjects underwent an intensive one-session exposure treatment along the lines of Öst (1989). Before and after the treatment session subjects completed the SPQ. The SPQ is a validated 31 item self-report instrument that measures fear of spiders (Fredrikson, 1983); SPQ scores can range from 0 to 31 . Immediately after the treatment session, subjects also underwent an illusory correlation paradigm (see below) to assess residual covariation bias. After two years, subjects were invited to complete the SPQ a third time. From the 19 phobic subjects who participated in that study, 13 subjects $(68 \%)$ returned the SPQ at follow up. The 6 remaining subjects were moved to an unknown address and could not be traced. The treatment effects as indexed by self reported fear and behavioral measures were similar for subjects who returned and subjects who did not return the follow-up SPQ.

\section{Procedure and Data Reduction}

In the study of de Jong and Merckelbach (1993), subjects were exposed to a series of 72 slides. There were three different categories: slides of flowers, weapons, and spiders. Immediately at slide offset one of three outcomes occurred: a shock, a siren, or nothing. All slide/outcome combinations were presented equally often, the conditional probability of a particular outcome 
given a certain slide being exactly $1 / 3$. After the experiment subjects were asked to rate to what extent each of the slides was followed by each of the outcomes (ranging from $0=$ not at all to $100=$ always). For each subject the sum of the three a posteriori reported stimulus/shock estimates was set at $100 \%$, to facilitate comparison between subjects regarding their spider/shock covariation estimates. Thus, covariation bias was expressed as the spider/shock estimate divided by the sum of all three stimulus/shock estimates. Relapse was indexed by $\mathrm{SPQ}_{\text {follow up }}$ minus $S P Q_{\text {post-reatment }}$ (i.e., higher scores indicate larger relapse). To test the hypothesis under consideration, a Pearson p-m correlation was computed between covariation bias and relapse.

\section{Results and Discussion}

\section{Treatment Effects}

In general the treatment yielded good immediate and long-term results. Mean SPQ decreased from 22.4 during the pre-treatment assessment to 11.2 after the second treatment session, $\mathrm{t}(12)=7.4, \mathrm{p}<.001$. By and large, this effect was maintained at two years follow up; for the subjects who returned the final SPQ, the mean follow up score was 11.4 (see Table 4.1).

\section{Covariation Bias and Relapse}

Correlational analysis revealed a significant positive correlation between relapse and covariation bias $(r=.61, \underline{p}<.05, \underline{n}=13)$. That is, the larger the covariation bias immediately after treatment, the larger the relapse at 2 years follow up. The present correlation between covariation bias and relapse is not mediated by residual fear immediately after treatment (as indexed by the posttreatment SPQ). If the influence of the post-treatment SPQ score is partialled out, the correlation remains unaffected. This result provides an empirical basis for the alleged reciprocal relationship between covariation bias and phobic fear. Thus, the present data confirm the hypothesis that covariation bias may enhance fear, thereby contradicting the suggestion that cognitive biases are merely epiphenomena of fear.

Table 4.1: Pre-treatment and post-treatment SPQ scores, mean covariation bias immediately after treament, and SPQ scores at 2 years follow up. Standard deviations are given berween parentheses.

\begin{tabular}{cccc}
\hline $\mathrm{SPQ}_{\mathrm{pre}}$ & $\mathrm{SPQ}_{\mathrm{pas}}$ & Covariation Bias & $\mathrm{SPQ}_{\text {tollow up }}$ \\
\hline $22.4(6.4)$ & $11.2(7.7)$ & $.47(.17)$ & $11.4(7.6)$ \\
\hline
\end{tabular}

The present findings are in line with the conviction that overestimation of 
threat plays a causal role in the origens and maintenance of irrational fears (e.g. Hawton, Salkovskis, Kirk, \& Clark, 1989). More specifically, the data suggest that relapse can be reasonably well predicted by a residual, posttreatment tendency to overestimate the likelihood that phobia relevant cues will be followed by aversive events. Note that the results of the present study fit nicely with recent findings of Margraf and Schneider (1993) who showed that there is a strong relationship between long term treatment succes in panic patients and panic-specific cognitive changes (e.g., the perceived threat of physical symptoms).

Whereas a large number of studies have demonstrated that phobic fear is accompanied by cognitive biases such as attentional bias (e.g., Lavy, van den Hout, \& Arntz, 1993) and covariation bias, very few studies have addressed the causal sequences of anxiety and biases (Logan \& Goetsch, 1993). There is strong evidence to suggest that cognitive biases disappear once anxiety is successfully treated, but this, of course, does not preclude the possibility that anxiety induces cognitive bias rather than vica versa (see Merckelbach, van Hout, De Jong, van den Hout, 1990). However, a recent study by Macleod and Hagan (1992) showed that a measure of attentional bias is better able to predict subsequent development of dysphoric reaction to stress than are traditional questionnaire indices. This result together with the present findings strengthen the idea that cognitive biases play a causative role in the development of anxiety. Given the lack of reliable, theory-derived predictors of anxiety relapse (or development) and given the clinical relevance of such predictors, efforts to replicate these findings would be welcomed.

Furthermore, it would be interesting to examine how attentional bias and covariation bias are related to each other. Is the former bias operating at a lower perceptual level and the latter at the higher level of judgmental processes? (see Mineka \& Sutton, 1992), or are both biases manifestations of a "top down" bias in the decision mechanism that assigns processing priorities (see e.g., Macleod \& Mathews, 1991)? Studies measuring both types of cognitive bias could provide an answer. 


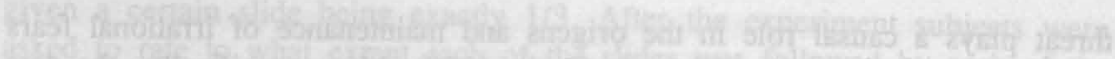

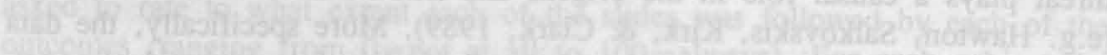

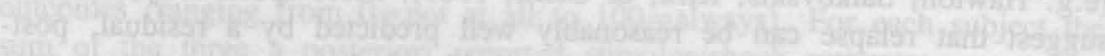

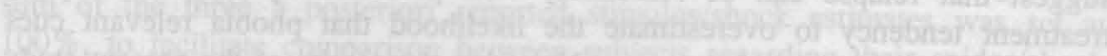

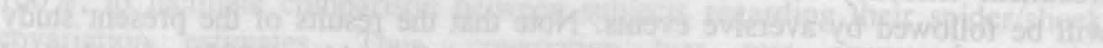

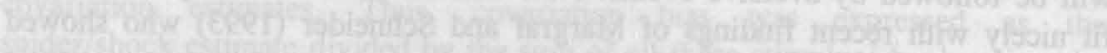

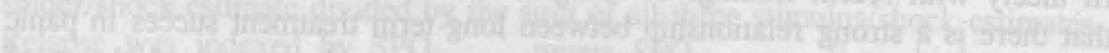

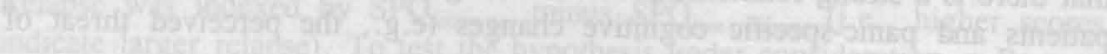

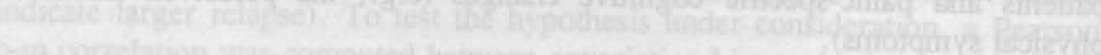

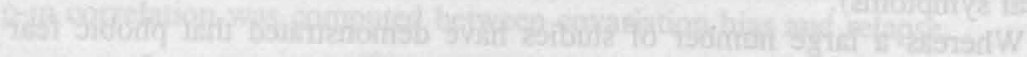

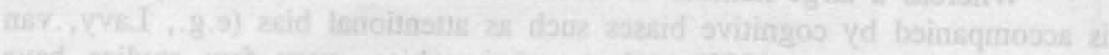

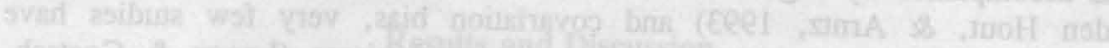

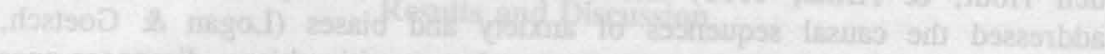

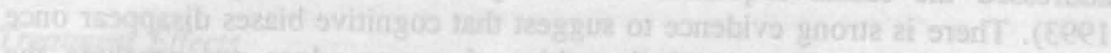

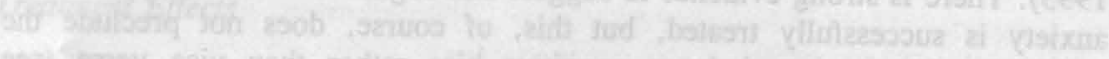

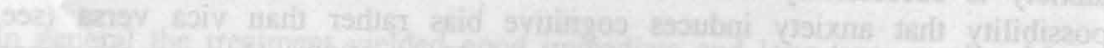

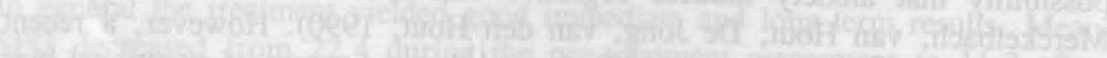

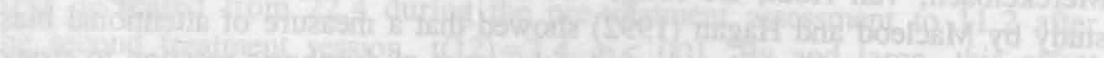

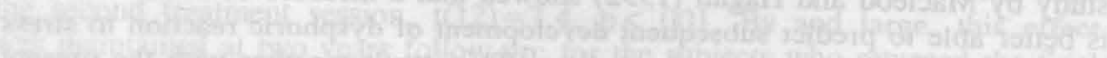

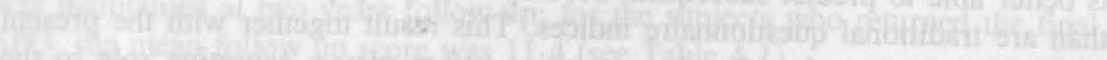

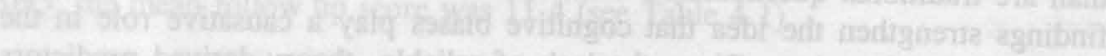

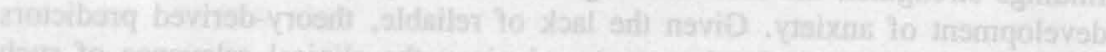

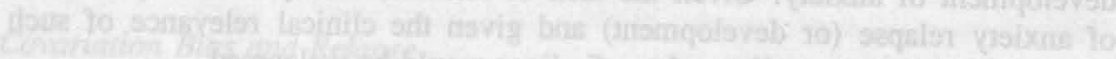

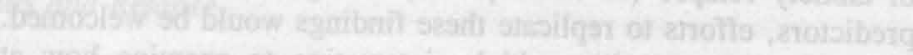

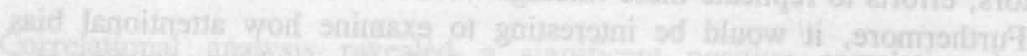

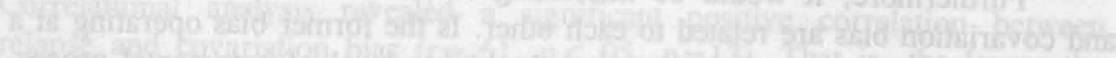

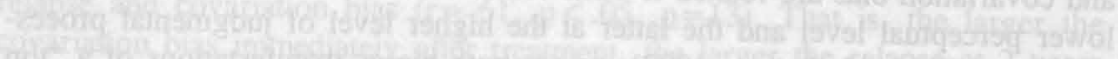

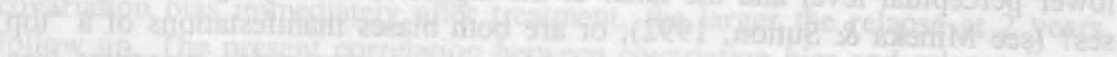

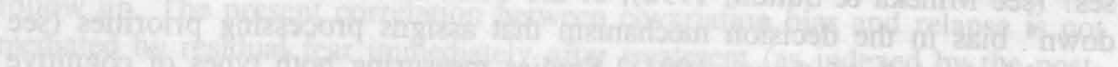

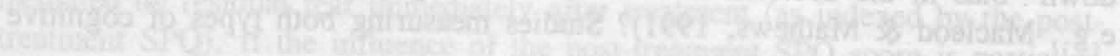

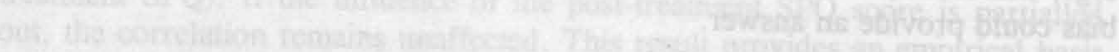

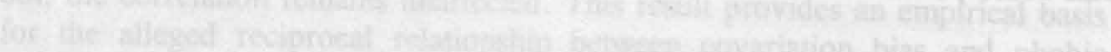

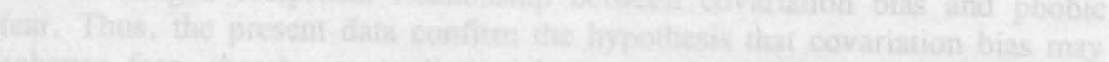

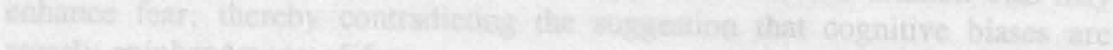

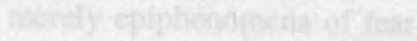

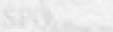


5.a THE PHENOMENA II: UCS-REVALUATION AND PHOBIA

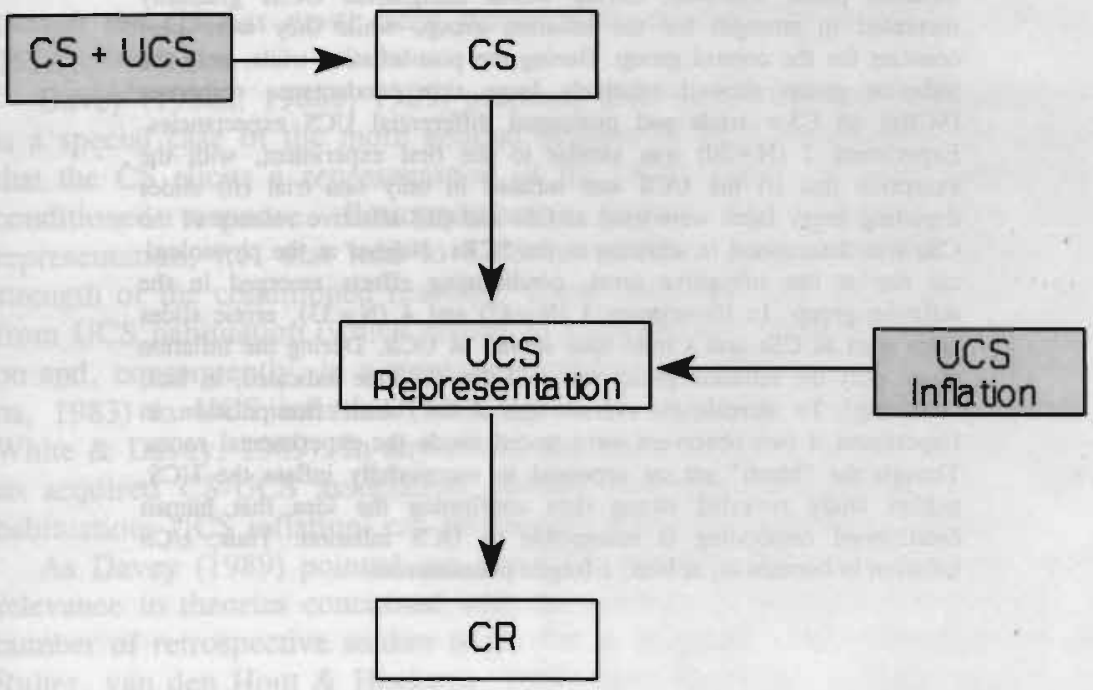

This chapter is partially based on papers presented at the First European Congress on Psychophysiology (Tilburg, 1991) and the European Congress on Behaviour and Cognitive Therapies, 1993 (September, London). A modified version of this chapter is published as: De Jong. P.J., Merckelbach, H., Koertshuis, G., \& Muris, P. (1994). UCS Inflation and Acquired Fear Responses in Human Conditioning. Advances in Behaviour Research and Therapy, in press.

Ackmowledgements: The authors thank Henk Nijman for his assistance during the pilot studies of the reported experiments. 


\title{
UCS Inflation and Acquired Fear Responses in Human Conditioning
}

\begin{abstract}
Four experiments concerning UCS-inflation in humans were conducted employing a differential conditioning paradigm. In Experiment 1 $(\mathrm{N}=30)$ one neutral slide (CS + ) was paired with a mild electric shock (UCS) and another neutral slide (CS-) was never paired with shock. An inflation phase followed, during which unsignalled UCSs gradually increased in strength for the inflation group, while they were kept constant for the control group. During the post-inflation trials, only the inflation group showed relatively large skin conductance responses (SCRs) on CS + trials and prolonged differential UCS expectancies. Experiment $2(\mathrm{~N}=20)$ was similar to the first experiment, with the exception that (i) the UCS was inflated in only one trial (ii) slides depicting angry faces were used as CSs and (iii) affective valence of the CSs was determined in addition to the SCRs. Neither at the physiological nor at the subjective level, conditioning effects emerged in the inflation group. In Experiment $3(\mathrm{~N}=42)$ and $4(\mathrm{~N}=33)$, erotic slides were used as CSs and a mild tone served as UCS. During the inflation stage, only the inflation group was told that the tone indicated, in fact, "blushing". To increase the aversiveness of the "blush" manipulation, in Experiment 4 two observers were seated inside the experimental room. Though the "blush" set up appeared to successfully inflate the UCS, neither study revealed strong data confirming the idea that human conditioned responding is susceptible to UCS inflation. Thus, UCS inflation in humans is, at best, a fragile phenomenon.
\end{abstract}

Animal studies have demonstrated that a postconditioning increase in the intensity of the unconditioned stimulus (UCS) may strengthen conditioned responding to a conditioned stimulus (CS) during a subsequent test phase (e.g., Bouton, 1984; Hendersen, 1985; Rescorla, 1974). That is to say, when animals are first confronted with pairings of a CS and a mild UCS and then are given some unsignalled, strong UCSs (UCS inflation), their conditioned reactions to further presentations of the CS are enhanced.

Thus far, only a study by White and Davey (1989) has examined the UCSinflation phenomenon in human conditioning ${ }^{1}$. Using a differential conditioning set-up, White and Davey (1989) measured skin conductance responses (SCRs) to neutral visual stimuli. During an initial acquisition phase, some stimuli $(\mathrm{CS}+)$ were followed by a mild, non-aversive tone $(65 \mathrm{~dB})$, while other stimuli (CS-) were never followed by a tone. Next, half of the subjects

${ }^{1}$ There are some human studies employing UCS revaluation [e.g., Baeyens et al., 1992; Siddle et al. 1988; Poulus et al., 1979]. However, a core feature of those studies was that they used UCS revaluation procedures as a tool for testing other theoretical notions, thereby taking for granted that UCS revaluation is a robust phenomenon in human conditioning. 
(inflation group) were confronted with a series of unsignalled tone UCSs that gradually became stronger in intensity and eventually reached the aversive 115 $\mathrm{dB}$ level. The control group was confronted with UCSs that were kept constant at the $65 \mathrm{~dB}$ level. During the final extinction phase, all subjects were given test presentations of the $\mathrm{CS}+$ and the CS-. Whereas differential electrodermal responding (i.e., stronger SCRs to $\mathrm{CS}+\mathrm{s}$ than to CS-s) was absent during the initial acquisition phase, it did occur during the final extinction phase, but only so in the inflation group. Accordingly, the authors concluded that " $a$ differential conditioned response can be acquired even though the CS has never been paired with an explicitly aversive UCS" (p. 265).

Davey $(1988 \mathrm{a}, 1988 \mathrm{~b}, 1989)$ proposes that the UCS-inflation phenomenon is a special case of the more general process of UCS revaluation. He argues that the CS elicits a representation of the UCS, which, in turn, induces the conditioned response. Postconditioning factors that influence this UCS representation, i.e. that lead to UCS revaluation, necessarily also affect the strength of the conditioned reaction. These postconditioning factors can range from UCS habituation (which results in a devaluation of the UCS representation and, consequently, in a weakened conditioned reaction (Davey \& McKenna, 1983) to UCS inflation (resulting in an enhanced conditioned response; White \& Davey, 1989). In any event, the important point to note is that given an acquired CS-UCS association, further non-associative events (e.g., UCS habituation; UCS inflation) can profoundly affect the conditioned response.

As Davey (1989) pointed out, the UCS inflation phenomenon might be of relevance to theories concerned with the etiology of phobias. For example, a number of retrospective studies (e.g., Öst \& Hugdahl, 1981; Merckelbach, de Ruiter, van den Hout \& Hoekstra, 1989) have found that a slight majority of phobic patients attribute their phobia to an aversive conditioning experience. Meanwhile, a considerable number of patients (e.g., $15 \%$ in the Öst \& Hugdahl study; $14 \%$ in the Merckelbach et al. study) can not recall an aversive event that was connected to the onset of their phobia. One could, of course, as some authors have done (e.g., Murray \& Foote, 1979), consider these cases as clear-cut examples that refute a conditioning interpretation of phobias. Yet, it may well be that the phobias of these patients stem from a more subtle conditioning process, e.g. a UCS-inflation scenario.

\section{Clinical Intermezzo}

To illustrate that UCS revaluation processes may play a prominent role in the aetiology of anxiety disorders, three annotated case histories will be presented which apparently follow an UCS-inflation scenario (see for a more extensive comment on these clinical cases Davey, de Jong \& Tallis, 1993): 
anxiery was mostly accompanied by physical symptoms. The most salient symptom was intestinal unease. On one occasion, when L.L. was alone at home and not anxious at all, similar symptoms of intestinal unease led to an uncontrolable attack of diarrhoea. From that moment on, L.L. catastrophically interpreted the symptoms of intestinal unease that he regularly experienced in company as a signal for losing control again. He became extremely anxious and developed severe agoraphobic symptoms.

Comment. This case can be considered as a clinical analogue of the UCS-inflation experiment of White and Davey (1989). First the intestinal unease (mild UCS) was associated with being in social company (CS). Then, an isolated UCS experience inflated the aversiveness of the intestinal unease. Apparantly as a result, the CS acquired threatening properties and subsequently L.L. attempted to avoid the CS (i.e., social situations).

Case 2. H.B. (female, aged $35 \mathrm{yr}$ ) applied for therapy complaining of a severe spider phobia (DSM-III-R criteria of simple phobia). Because of her father's occupation, she had lived in Rio de Janeiro in Brazil during her childhood. Once, at the age of $10 \mathrm{yr}$, she woke at night when a large tropical spider walked over her face. At the time she reported not being particularly frightened. However, when she told her parents about the incident the following morning, they were extremely concerned and looked very alarmed. From that moment on, H.B. was extremely frightened of spiders and exhibited severe phobic behaviour.

Comment. There is no a priori reason to assume that only inflated UCS experiences can result in UCS revaluation. This case exemplifies how socially/verbally transmitted information rather than experience per se, increased the aversiveness of the UCS (that was first experienced as fairly mild) and how this information eventually led to phobic fear for the CS (i.e., spider) supposedly by activating a representation of the inflated UCS.

Case 3. F.G. (male, aged $46 \mathrm{yr}$ ) suffered from irregular heartbeats and palpitations for more than 20 yr. F.G. stressed that he had never been anxious because of these physical symptoms. When his father was $49 \mathrm{yr}$ old, F.G's father died from a heart attack. At the time of his father's death, F.G did not develop any anxiety symptoms. However, when F.G. reached the age at which his father had died of the heart atrack, he became very anxious and began to catastrophically interpret his palpitations as predictors of a hear attack. He was subsequently diagnosed as suffering from panic disorder.

Comment. This case represents an example of yet another route for UCS inflation: Reappraisal of subjects' own responses. Originally, the bodily symptoms (CS) were not particularly threatening. however, at the age at which such symptoms were fatal for his father, the aversiveness of the consequences (UCS) associated with the bodily symptoms were dramatically inflated. In passing, note that this conceptualisation of bodily symptoms acquiring phobogenic properties nicely fit with the cognitive approach of panic (e.g.. Clark, 1988) in which catastrophic interpretation of bodily symptoms is a central feature.

In sum, then, it is sometimes possible to describe the etiology of particular anxiety disorders in terms of an inflation scenario. This may not only improve our understanding of the aetiology of anxiety disorders, it may also have clinical implications for the treatment of such disorders. However, it should be stressed that such seemingly convincing cases are gratuitous as long as there is no solid and independent empirical evidence available indicating that UCS inflation does, indeed, affect human fear conditioning. Therefore, with the potential clinical relevance of the UCS-inflation phenomenon in mind, a series of laboratory experiments were carried out in order to cross-validate and extend the previous findings of White and Davey (1989). 


\section{Experiment 1}

In the first experiment, the effect of postconditioning UCS inflation on human 'fear' conditioning was examined. An electric shock rather than a tone (White \& Davey, 1989) served as UCS. As in the experiment of White and Davey the impact of the inflation procedure on electrodermal responding was examined. However, to be a good minimodel (Marks, 1977) of the etiology of phobias, the inflation procedure should not only affect physiological responding to the CS + , but also increase subjective UCS expectancies on CS + trials; If, as Davey proposes, UCS inflation affects the UCS representation that the subject connects to the $\mathrm{CS}+$, than one predicts that subjective expectancies with regard to UCS occurrence are sensitive to a UCS inflation manipulation. In clinical terms: catastrophic expectancies related to the CS+ accompany phobic fear and avoidance and in case of an UCS inflation scenario, subjects display such expectancies eventhough they might be unable to recall any actual CS+/UCS coincidences. Thus, to investigate further the validity of the UCS inflation procedure, also subjects' on-line UCS expectancies were examined in the present experiment.

\section{Method}

\section{Subjects}

Thirty undergraduate students (21 women) participated in the experiment in return for a small financial compensation. Their mean age was 22 years (range: $18-27$ years.).

\section{Apparatus and Stimulus Material}

Skin conductance responses (SCRs) were measured with a Beckman Skin Conductance Coupler (type 9844), using the method of constant voltage $(0.5$ volts). The coupler was connected to two Beckman $\mathrm{Ag}-\mathrm{AgCl}$ electrodes (diameter $8 \mathrm{~mm}$ ) that were filled with isotonic paste and attached to the medial phalanges of the second and third finger of the left hand. Respiration was measured by means of a Beckman respiration belt fastened around the subject's chest. The respiration belt was connected to a Beckman Pressure/Pulse/Volume coupler (type 9878). SCRs and respiration were continuously monitored on a Beckman R611 polygraph.

Slides depicting neutral stimuli (i.e., mushrooms and flowers) served as CSs. They were presented with a Kodak Carousel and projected onto a white screen, approx. $2.5 \mathrm{~m}$. in front of the subject. The size of the projected image was approx. $80 \times 120 \mathrm{~cm}$.

Electric shocks served as UCSs. A specially designed shock generator (0$40 \mathrm{~mA}$ ) was used for administration of the shock UCSs. The shocks (dc) were 
delivered through two electrodes attached to the medial phalanges of the second and third finger of the right hand. UCS expectancies were measured by means of a dial that could be rotated either to the left or to the right, with $90^{\circ}$ to the left meaning "certain that shock will not occur" and $90^{\circ}$ to the right meaning "certain that shock will occur". The dial was connected to a Beckman Pressure/Pulse/Volume coupler (type 9878) that was calibrated in such a way that $-20 \mathrm{~mm}$ pen deflection corresponded to "certain that shock will not occur", while $+20 \mathrm{~mm}$ pen deflection corresponded to "certain that shock will occur". A PDP Minc-11 microcomputer controlled CS presentation, UCS administration, intertrial intervals, and response registration.

\section{Design}

The experiment was designed according to a 2 (group) $\times 2$ (reinforcement) factorial model. The first between-subject factor refers to the fact that half of the subjects (inflation group) were confronted with a postconditioning inflation of the shock UCS, whereas the other half of the subjects (control group) were confronted with postconditioning UCSs that were kept at a constant (low) level. The second, within-subject, factor pertains to the fact that all subjects saw two slides, one of which (CS+) was paired (during acquisition) with a mild UCS, while the other (CS-) was never paired with the UCS.

\section{Procedure}

Subjects sat in a comfortable chair that was placed in a dimly lit, soundattenuated chamber. Apparatus was placed in an adjoining room. After recording sites had been cleansed with distilled water, electrodes and respiration belt were attached. Next, subjects underwent a shock work-up procedure in which the shock intensity was gradually increased. For each subject, two shock intensity levels were determined: a (low) threshold intensity and a high intensity that was evaluated as "uncomfortable but not painful".

Subjects were then given instructions about the use of the UCS-expectancy dial. It was stressed that they should express their expectancy of shock on a moment to moment basis throughout the experiment. Subjects were not informed about the CS+/UCS contingency, nor were they instructed about the occurrence of inflated or non-inflated UCSs. The experiment proper consisted of three phases. The first was an acquisition phase that involved $6 \mathrm{CS}+/ \mathrm{UCS}$ pairings and $6 \mathrm{CS}$ - presentations. An inflation phase followed during which 4 unsignalled UCSs were administered. The final extinction phase consisted of 6 unreinforced CS + and $6 \mathrm{CS}$ - trials. Slide duration was $6 \mathrm{sec}$. The sequence of CS + and CS- slides was quasi-random, with the restriction that no more than two successive presentations of the same slide occurred. The type of slide that served as CS + and CS- was counterbalanced across subjects. Intertrial intervals ranged from 10 to $20 \mathrm{sec}$, with a mean of $15 \mathrm{sec}$. No interruptions 
beyond this mean intertrial interval occurred between the three phases of the experiment. UCS duration was 0.5 sec.. During acquisition, UCS intensity was approximately $10 \%$ above the threshold level and UCSs were presented immediately at CS+ offset. For the inflation group, UCS intensity was increased in three steps during the inflation phase. For the control group, UCS intensity was kept constant.

\section{Data Reduction and Analysis}

SCRs were defined as maximal deflections occurring 1-4 sec. after CS or UCS onset. SCRs were measured in micromho and subjected to a rangecorrection transformation (Lykken, 1972) to aid comparison of responses across subjects: Each subject's SCR was weighted by that subject's maximal response during the acquisition phase.

Respiration was used as a control variable. SCR trials with respiratory irregularities were excluded from statistical analyses. Such irregularities occurred on less than $2 \%$ of the trials. SCR values for these trials were estimated on the basis of the SCRs on adjacent trials.

UCS-expectancy data were obtained by measuring $\mathrm{mm}$ pen deflections during CS + and CS- trials ranging from $-20 \mathrm{~mm}$ ("certain that shock will not occur") to $+20 \mathrm{~mm}$ ("certain that shock will occur").

For both SCRs and UCS-expectancies, CS-/CS + difference scores were computed and subjected to MANOVA trend analyses. The mean and linear trends were inspected. The mean trend was analyzed to investigate differential responding to CS + and CS- trials. The linear trend was inspected to evaluate whether differential responding increased/decreased across trials. A group factor (inflation/control) was included to evaluate whether the pattern of results differed between groups.

\section{Results}

\section{UCS Intensity}

Table 5.1 shows the UCS intensities that were used during the acquisition and the inflation phase. Shock intensities for the inflation and control group did not differ during the acquisition phase $[\mathrm{t}(28)<1.0]$. In contrast, the fourth shock administered during the inflation phase was significantly stronger in the inflation than in the control group [ $\mathrm{t}(28)=5.4, \mathrm{p}<0.01$, one-tailed].

The difference in UCS intensity between both groups is also reflected in the magnitudes of the UCRs. Unconditioned SCRs during inflation (i.e., SCRs to the isolated UCSs) became larger in the inflation group as a result of the gradually increasing shock intensities. In contrast, unconditioned SCRs in the control group habituated due to the low and stable UCS intensities with which this group was confronted during the inflation period (see Table 5.2). A 2 
(group) $\mathrm{x} 4$ (UCS presentation) ANOVA yielded, indeed, a significant interaction of group with UCS presentation $[\mathrm{F}(1,28)=5.10, \mathrm{p}<0.01]$. Thus, the inflation manipulation succeeded in that stronger reactions were evoked in the inflation than in the control group.

Table 5.1: Mean UCS-intensities (mA) during acquisition and inflation (fourth presentation) for both groups. Standard deviations are given between parentheses.

\begin{tabular}{lll}
\hline & Acquisition & Inflation (4th UCS) \\
\hline Control group & 0.17 & 0.17 \\
& $(0.23)$ & $(0.23)$ \\
\hline Inflation group & 0.22 & \\
& $(0.32)$ & 3.75 \\
& & $(0.98)$
\end{tabular}

Table 5.2: Mean unconditioned SCRs (range-corrected) to the 4 shock UCSs of the inflation phase for both groups. Standard deviations are given between parentheses.

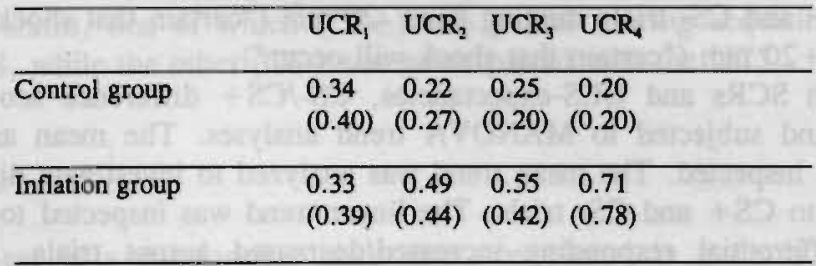

\section{SCRs}

The left panels of Figure 5.1 show SCRs to CS+ and CS- slides during acquisition. The MANOVA revealed a multivariate effect of conditioning, $\mathrm{F}(2,27)=9.48, \mathrm{p}=.001$. Univariate $\mathrm{F}$-tests indicated that there was a mean trend as well as a linear trend, $\underline{F}(1,28)=14.7, \underline{p}<.01$ and $\underline{F}(1,28)=7.1$, $\mathrm{p}<.05$, respectively. The mean trend confirms that SCRs to CS + slides were larger than those to CS- slides and the linear trend indicates that this differential responding increased over trials. Although Figure 5.1 suggests that differential responding was stronger in the control group than in the inflation group, statistical analysis showed that there was no significant difference between groups in this respect. This pattern of results did not differ between groups. That is, no interaction with group emerged, $F(2,27)<1$.

The right panels of Figure 5.1 show SCRs to CS + and CS- during extinction. A MANOVA showed a multivariate effect of conditioning, $\mathrm{E}(2,27)=4.26, \mathrm{p}<.05$. A univariate $\mathrm{F}$-test yielded a significant mean trend, indicating a main effect of conditioning: Also during extinction, SCRs to CS+ 
slides were larger than those to CS- slides, $F(1,28)=6.88, \mathrm{p}<0.05$. The linear trend was also significant, indicating that the conditioning effect was particularly strong on the first extinction trials, $\mathrm{F}(1,28)=5.67, \mathrm{p}<0.05$.

Most interestingly, the mean trend was different for both groups, $\mathrm{F}(1,28)=4.24, \mathrm{p}<.05:$ In line with the hypothesis, the remaining effects of conditioning were stronger in the inflation group than in the control group. Accordingly, a significant mean trend was evident within the inflation group but not within the control group, $\underline{F}(1,28)=10.9, p<.01$ and $\underline{F}(1,28)=0.16$, $\mathrm{p}>.50$, respectively. Both groups did not differ with regard to the linear trend, $\mathbf{F}(1,28)<1$, indicating that the differences between the CRs on the $\mathrm{CS}+$ and the CS- trials extinguished for both groups at a similar rate.
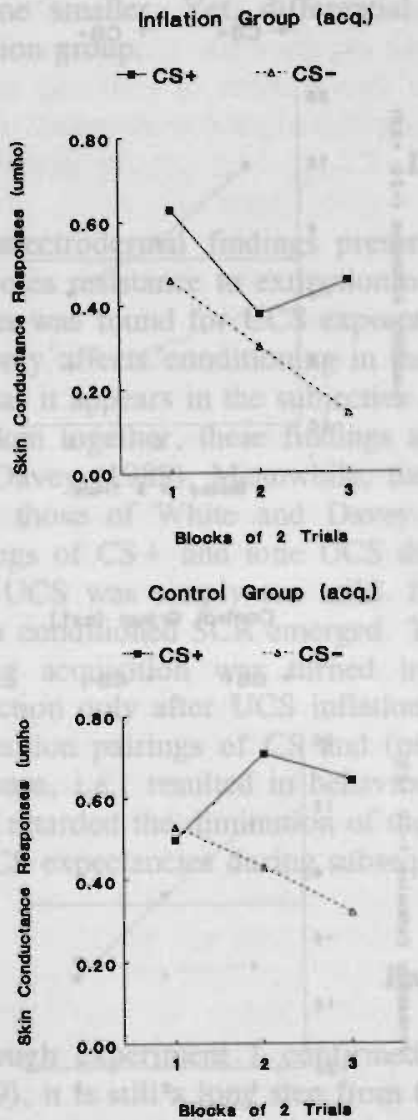

Inflation Group (ext.)

$\rightarrow-\mathrm{cs}^{+} \quad \mathrm{cs}-$

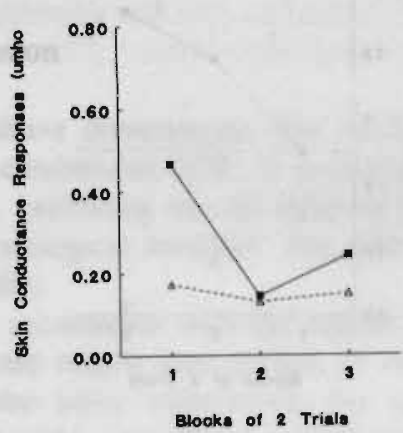

Control Group (ext.)

$-\mathrm{cs}+\quad \mathrm{cs}-$

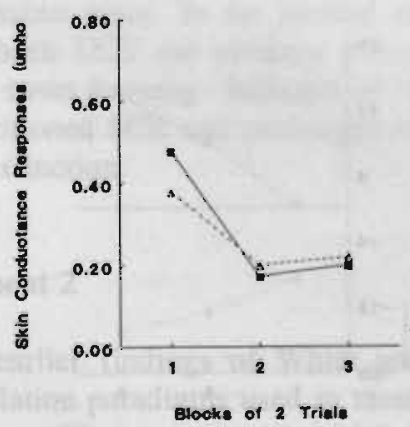

Figure 5.1: Skin conductance responses (SCR/SCR acquisition (left panel) and extinction (right panel) in blocks of two trials. 


\section{UCS Expectancy}

Mean UCS-expectancy scores during acquisition are shown in the left panels of Figure 5.2. The trend analysis revealed a multivariate effect of conditioning, $\underline{\mathrm{F}}(2,27)=77.74, \mathrm{p}<.001$. Univariate $\underline{\mathrm{F}}$ tests revealed both a significant mean trend and a significant linear trend, $\mathrm{F}(1,28)=117.7, \mathrm{p}<0.001$ and $\mathrm{F}(1,28)=65.3, \mathrm{p}<.001$. Thus, UCS expectancies were stronger after CS + trials than after CS- trials and the differential UCS expectancies increased over trials. No group interaction emerged, $F(2,27)=1.01, p>.30$. In other words, during the acquisition period differential UCS expectancies appeared to be equally strong for both groups.
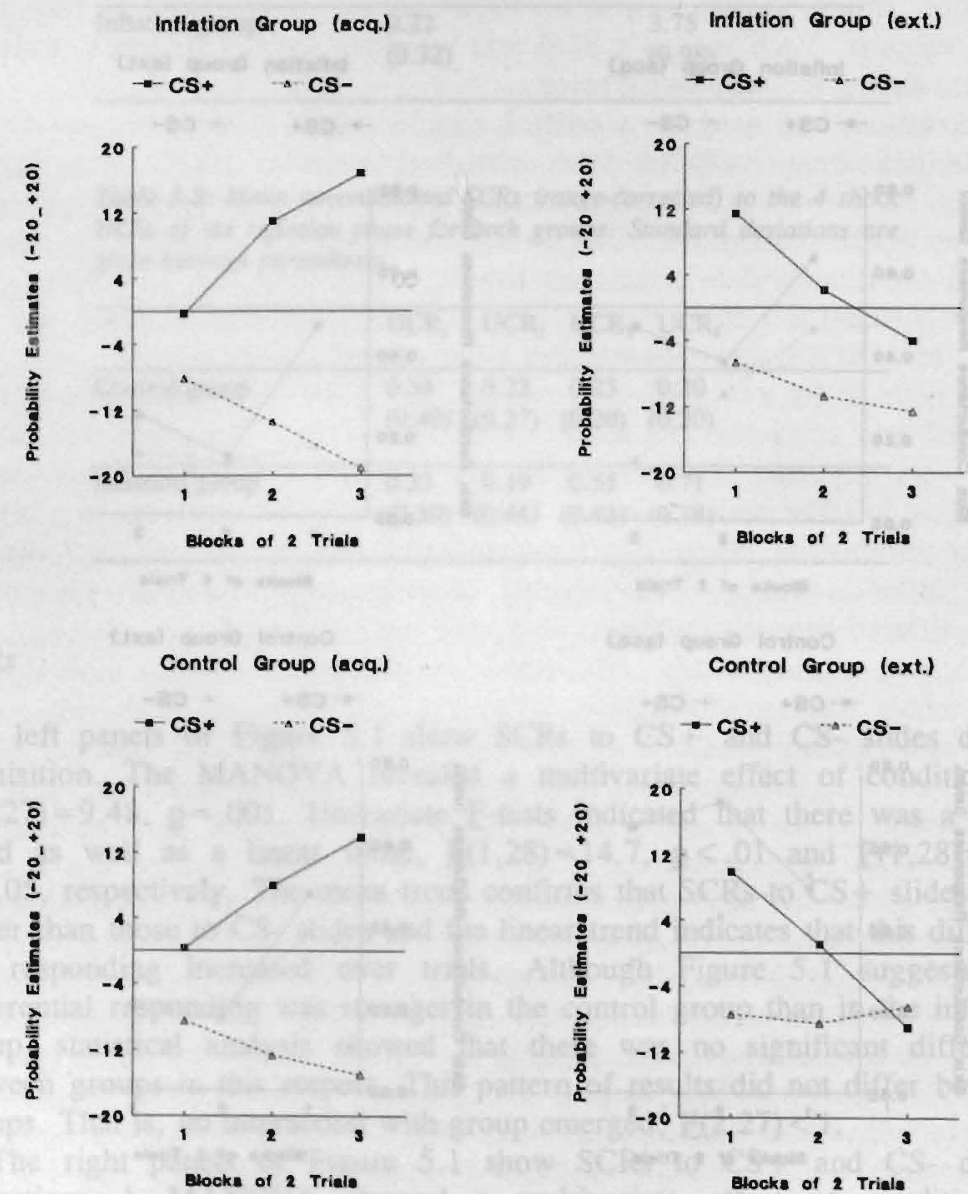

Figure 5.2: On-line probability estimates of shock outcome during the CS+ and CS-trials for the acquisition (left panel) and extinction (right panel) in blocks of two trials. 
UCS expectancies during extinction are shown in the right panel of Figures 5.2. The trend analysis yielded, again, a significant multivariate effect of conditioning $\underline{F}(2,27)=25.1, \mathrm{p}<.001$. Furthermore, univariate $\underline{\mathrm{F}}$ tests revealed a significant mean trend $\mathrm{F}(1,28)=26.4, \mathrm{p}<0.001]$ and a significant linear trend $\underline{F}(1,28)=42.2, p<0.001]$ : UCS expectancies were greater on CS + than on CS- trials, but this difference became smaller over trials. Most pertinent to the hypotheses under consideration was a multivariate effect of the experimental manipulation, $\underline{F}(2,27)=4.6, \underline{p}<.05$. That is, the conditioning effect was different between groups. A univariate $\underline{F}$ test showed that the difference of the mean trends between both groups did not reach significance $\underline{E}(1,28)=1.5, p=$ .21. Yet, the linear trend differed significantly between both groups $\mathrm{F}(1,28)=5.22, \mathrm{p}<.05$. In other words, both groups started with stronger UCS expectancies on CS + than on CS- trials and this difference gradually became smaller. Yet, differential UCS expectancies survived longer in the inflation group.

\section{Discussion}

The electrodermal findings presented above demonstrate that UCS inflation promotes resistance to extinction of the conditioned SCR. A similar pattern of results was found for UCS expectancies, indicating that an inflation procedure not only affects conditioning in the physiological modality, but also conditioning as it appears in the subjective modality.

Taken together, these findings are in accordance with the results of White and Davey (1989). Meanwhile, the current results also deviate in one respect from those of White and Davey. In the latter experiment, the acquisition pairings of CS + and tone UCS did not yield a conditioned SCR because the tone UCS was simply too mild. It was only after inflation of the tone UCS that a conditioned SCR emerged. Thus, a behaviourally silent learning process during acquisition was turned into an overt conditioned response during extinction only after UCS inflation had taken place. In the present study, the acquisition pairings of CS and (mild) shock UCS did produce a conditioned response, i.e., resulted in behaviourally overt learning. Inflation of the shock UCS retarded the diminution of the conditioned SCR and prolonged differential UCS expectancies during subsequent extinction.

\section{Experiment 2}

Although experiment 1 confirmed the earlier findings of White and Davey (1989), it is still a long step from the inflation paradigms used in these experiments to the real life phobias that these paradigms attempt to model. Note for instance that in both experiments neutral (e.g., triangles, flowers) rather than phobogenic (e.g., angry faces or spiders) stimuli served as CSs. In addition, UCSs were inflated in several steps. Yet, UCS-inflation scenarios as they 
of the CS- did not differ between groups, $t(17)<1$, means being 0.09 and 0.23 for the experimental and control group, respectively.

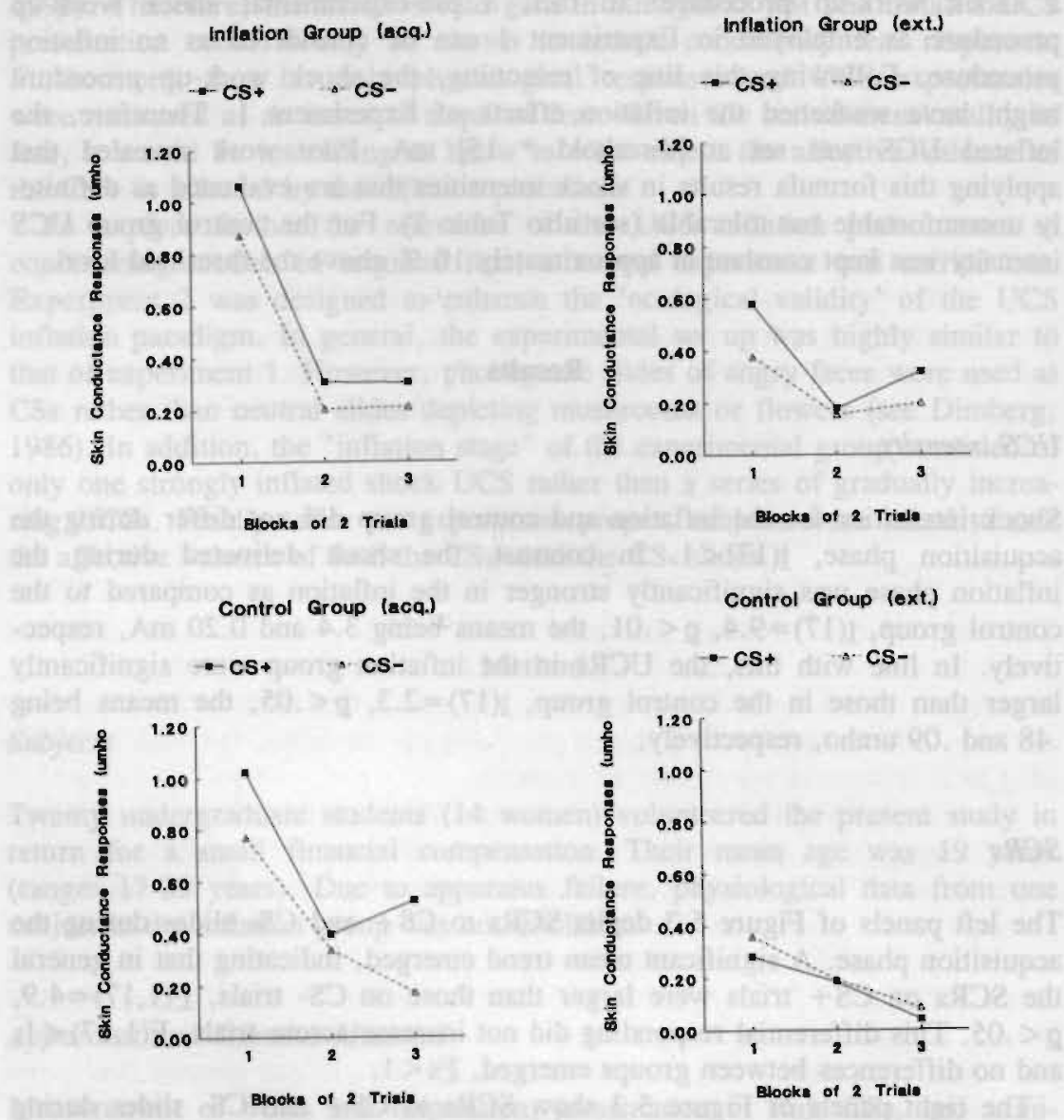

Figure 5.3: Skin conductance responses (SCR/SCR acquisition (left panel) and extinction (right panel) in blocks of two trials.

\section{Discussion}

The present study was designed to replicate and extend earlier experiments suggesting that human classical conditioning can be affected by UCS inflation procedures. In an attempt to improve the 'ecological validity' of Experiment 1 , several modifications were made. Phobogenic rather than neutral slides were used as CSs. In addition, only one inflated shock UCS was administered during the inflation period instead of a series of gradually increasing UCSs. Furthermore, the impact of the inflation procedure on subjects' appreciation of 
the slides as well as on their physiological reactions was investigated.

Yet, no differential extinction pattern between the inflation and the control group could be demonstrated in the present study: Inflation of the shock UCS did not retard the diminution of the conditioned SCR. In addition, the affective valence of the $\mathrm{CS}+$ slides was not affected by the inflation procedure.

Taken the available evidence together, one could argue that human conditioning is susceptible to UCS inflation, but only so under very specific (i.e., artificial) conditions. Alternatively, the absence of an inflation effect in the present experiment may be attributed to the UCS not being salient enough. That is, perhaps subjects were not that concerned about the isolated shock UCS. Possibly, one trial UCS inflation is only successful if the inflation is more intense and/or of personal relevance. To explore this possibility and to investigate further the generalizability of the UCS inflation phenomenon in human conditioning, a third experiment was conducted.

\section{Experiment 3}

The third experiment was designed to explore whether one instance of UCS inflation would be sufficient to induce conditioned responding in case the stimulus material is more salient. At the same time, the present study aimed at investigating further the generalizability of the phenomena under consideration. That is, the inflation model advanced by Davey (1989) would gain in strength if it could be shown that the UCS inflation phenomenon is not restricted to a highly specific laboratory manipulation, but can also be evoked with events that more closely approach real life.

One interesting event in this context is blushing. Blushing can be regarded as an aversive, interoceptive UCS. For example, several authors (e.g., Wolff, 1972; Newlin, 1989) have noted that there is a negative connection between skin-flushing responses and alcohol consumption in Asian individuals. Due to a specific liver dysfunction, a considerable percentage of Asian individuals blush after alcohol consumption. It has been suggested that these individuals develop an aversion of alcohol through a conditioning process in which alcohol (CS) becomes associated with the aversive qualities (i.e., social embarassment) of facial flushing (UCS). The idea that blushing functions as a UCS that elicits avoidance behavior can also be found among students of social phobia (e.g., Edelmann, 1990).

In experiment 3, erotic slides served as CSs. Mild non-threatening tone UCSs were used to establish a behaviourally silent acquisition phase. After the acquisition period, subjects in the inflation group were informed that the tone signalled "blushing". It was examined whether this UCS inflation/revaluation procedure resulted in conditioned SCRs during a subsequent test phase ("extinction") in which the erotic CSs occurred without tones. Furthermore, it was investigated whether UCS inflation affected subjects' evaluations of CS + and CS- slides. The hypothesis tested was that subjects in the inflation group would evaluate the CS + as more negative than the CS-, while subjects in the 
control group would not exhibit such difference.

\section{Method}

\section{Subjects}

Subjects were 42 undergraduate volunteers ( 17 women). They were paid for participating in this experiment. Mean age was 19.5 years (range 18-25 years).

\section{Apparatus and Stimulus Material}

Measurement of SCR and respiration, presentation of slides, computer control of response registration, CS presentation, UCS delivery, and intertrialintervals were identical to that of Experiment 1 and 2.

Erotic slides from the International Picture System (Lang, Öhman \& Vaitl, 1988 ) served as CSs. Men were shown two different slides depicting women and women were shown two different slides depicting men. A mild tone (250 $\mathrm{Hz}$, approx. $70 \mathrm{~dB}$ ) was used as UCS and was presented through a loudspeaker inside the experimental room.

\section{Design}

The experiment was designed according to a 2 (group) $\times 2$ (reinforcement) factorial model. The first between-subject factor refers to the fact that only half of the subjects (inflation group) were confronted with postconditioning inflation/revaluation information concerning the tone UCS. The second withinsubject factor pertains to the fact that all subjects saw two slides, one of which (CS+) was paired (during acquisition) with a mild tone UCS, while the other (CS-) was never paired with the UCS.

\section{Procedure}

Subjects sat in a comfortable chair that was placed in a dimly lit, soundattenuated chamber. Apparatus was placed in an adjacent registration room. After recording sites had been cleansed with distilled water, electrodes and respiration belt were attached. Next, a bogus electrode was placed on the left cheek, supposedly to measure the circulation of the skin. Subjects were told that the experiment was a pilot study to explore subjects' reactions to a series of slides that were planned to be used in a large scale project. It was explained that their task was just to look at a series of slides and that depending on the results, possibly a second series would follow. They were not informed 
about the UCS occurrences.

The experiment consisted of three phases. The first was an acquisition phase that involved $6 \mathrm{CS}+/ \mathrm{UCS}$ pairings and $6 \mathrm{CS}$ - presentations. An inflation stage followed. During this period, subjects in the inflation group were presented with audiotaped information regarding the tone UCSs that were paired with the CS+ slides:

"This was the first series of slides. At several points in time during the slide presentation you might have heard a tone coming out of the loud speaker. This tone is generated if the blood circulation of the skin exceeds a certain threshold level. Thus, if the circulation is above the threshold level for instance during blushing) there will be a tone until the circulation drops below the threshold level again. Anyway, this information has no implications for your task during the second series of slides that will start in a few moments. Again your task is to just watch the slides."

The control group was also provided with some audiotaped information during the second stage of the experiment. Yet, on this tape the "circulation" part was omitted.

The test ("extinction") phase consisted of 6 unreinforced CS + and 6 CStrials and was concluded with the presentation of an isolated tone UCS. This final tone was presented to test whether in the inflation group, the SCRs to the tone were enhanced due to the "inflation information".

After the experiment proper, subjects were asked to complete a questionnaire. This questionniare consisted of 5 visual analogue scales (VASs) and was designed to assess 1) the frequency of blushing in social situations (VAS ranging from $0=$ "very often" to "never" $=10$ ); 2) subjects' evaluation of blushing in social situations (ranging from $0=$ "very aversive" to 10 "very pleasant"); 3) and 4) subjects' appreciation of the CS + and CS- (ranging from $0=$ "very unpleasant" to $10=$ "very pleasant" (these ratings were made during a post-experimental presentation of the CS + and CS- slides); and 5) subjects' impression whether the tone appeared more often during one of the two slides (ranging from $0=$ "very sure that one of the slides was more often followed by a tone" to $10=$ "very sure that both slides were equally often followed by a tone"). VAS1 and VAS2 provided information as to whether subjects in both groups were comparable in respect to their "blushing history". In addition, VAS2 was used to examine whether subjects, indeed, experienced blushing as being aversive. VAS3 and VAS4 were included to test whether the inflation procedure differentially affected subjects' affective evaluation of the CS +. VAS5 was employed to check whether a CS +/UCS association was, indeed, established during the acquisition period.

Slide duration was 6 seconds. The sequence of slides was quasi- random, with the restriction that no more than two successive presentations of the same slide occurred. The type of slide that served as CS + or CS- was counterbalanced across subjects. To increase the credibility of the blushing instructions, the UCS tone had a variable onset and a variable duration. Tone onset varied 
from 4 to 6 seconds after slide-onset and the duration varied from 1 to 4 seconds.

\section{Results}

\section{SCRs}

The left panels of Figure 5.4 depict SCRs to CS+ and CS- slides during the acquisition phase. A MANOVA showed a multivariate effect of conditioning, $\underline{F}(2,39)=4.19, \underline{p}<.05$. A univariate $\underline{F}$ test yielded a significant mean trend, indicating that in general the SCRs on CS + trials were larger than those on CS- trials, $\mathrm{F}(1,40)=6.3, \mathrm{p}<.05$. This differential responding did not increase across trials, $E(1,17)<1$. Neither for the mean nor for the linear trend a group $x$ stimulus interaction emerged, $\underline{F}(1,40)=2.5, \mathrm{p}>.10$, and $\underline{F}(1,40)<1$, respectively.

Inflation Group (acq.)
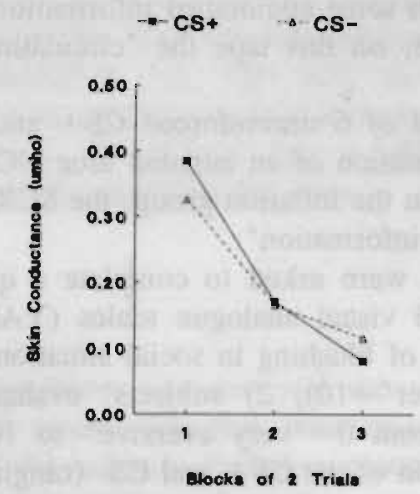

Control Group (aca.)

-cs+ $\cos -$

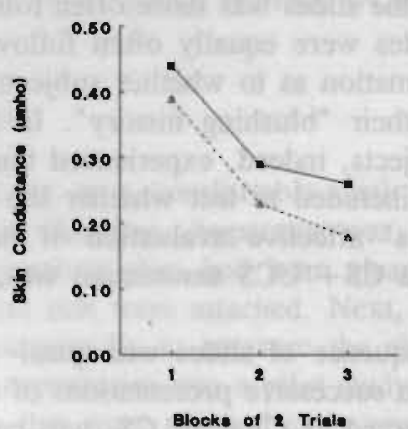

Inflation Group (ext.)
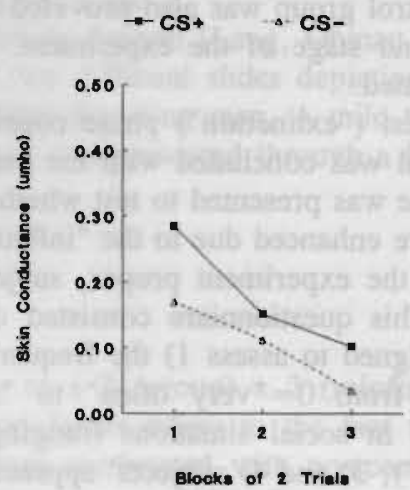

Blocks of 2 Trials

Control Group (ext.)

CS+ CS-

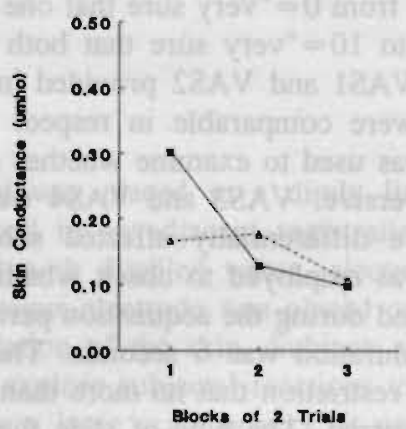

Figure 5.4: Skin conductance responses (SCR/SCR max $_{\text {andisition }}$ to $C S+$ and $C S$ - trials during acquisition (lefi panel) and "extinction" (right panel) in blocks of rwo trials. 
The right panels of Figure 5.4 show SCRs to CS + and CS- slides during extinction. The multivariate conditioning effect survived during extinction, $\mathrm{F}(2,38)^{2}=7.4, \underline{\mathrm{p}}<.01$. Univariate $\underline{\mathrm{F}}$ tests revealed a significant mean trend $[\underline{F}(1,39)=4.6, p<.05]$ as well as a significant linear trend $[\mathrm{F}(1,39)=5.7$, $\mathrm{p}<.05$ ], indicating that responses to CS + trials were larger than those to CStrials and that this differential responding declined over trials.

Neither the between group difference of the mean trends nor the between group difference of the linear trends reached conventional levels of significance, $\underline{F}(1,39)=1.7, \underline{p}=.20$, and $\underline{F}(1,39)=1.9, \underline{p}=.18$, respectively. Within group analyses revealed that only subjects in the inflation group showed differential responding between the CS + and the CS- slides. That is, in the inflation group, but not in the control group there was a significant mean trend, $\underline{F}(1,39)=5.6, \underline{p}<.05$ and $\underline{F}(1,39)=0.4, \underline{p}>.50$, respectively.

To further investigate the "inflation effect", difference scores were computed between the last two acquisition trials and the first two extinction trials (for both CS + and CS- trials). The difference scores were subjected to a trend analysis. The mean and the linear trends were inspected to test phase and phase $\mathrm{x}$ stimulus interaction effects, respectively. A group factor was included to test whether the pattern of results was affected by the experimental manipulation. The univariate $\underline{F}$ tests showed that the mean trend did but the linear trend did not differ between groups $\underline{F}(1,40)=6.3, p<.05$ and $\mathrm{F}(1,40)<1$, respectively. Yet, within-group analyses revealed a significant linear trend for the inflation group $[\mathrm{F}(1,40)=5.4, \mathrm{p}<.05]$ but not for the control group $[\mathrm{E}(1,40)=2.7, \mathrm{p}>.10]$. Thus, SCRs to both types of slides increased due to the inflation information. In addition, only in the inflation group, there was a significant phase $\mathrm{x}$ stimulus interaction; yet, this interaction did not significantly differ between both groups.

\section{UCS Inflation}

Figure 5.5 depicts the SCRs on the first tone UCS presented during the acquisition phase and the test tone that was presented at the end of the experiment. An ANOVA yielded a main effect of phase, $F(1,40)=15.5$. $\mathrm{p}<.01$, indicating that in general, reponses to the "test" tone were larger than those to the first tone during acquisition. Pertinent to the hypothesis under consideration, the ANOVA revealed a significant interaction between phase and group, $\underline{F}(1,40)=6.8, p<.05$, indicating that the "inflation" manipulation succeeded in inducing larger responses to the formerly neutral tone.

${ }^{2}$ due to apparatus failure for one subject extinction data are not complete. 


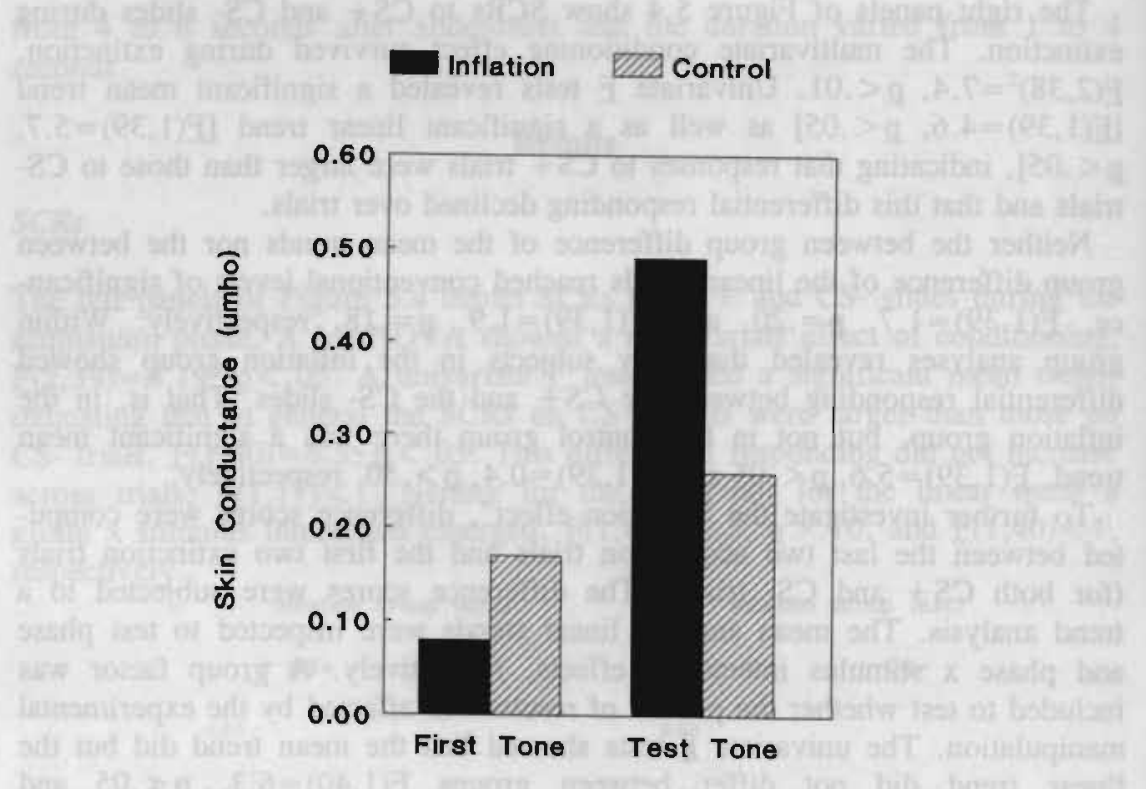

Figure 5.5: Mean SCR to the first tone UCS presented during acquisition and mean SCR to a test tone UCS presented at the end of the extinction phase for the inflation and the control group.

\section{Post-Experimental Questionnaire}

The questionnaire data revealed that subjects in both groups did not differ with regard to their "blushing history". That is, mean scores on VAS1 and VAS2 were comparable for both groups, $\mathrm{t}(40)=.15$ and $\mathrm{t}(40)=.16$, respectively. In addition, the VAS2 data confirmed that on the whole, subjects considered social blushing as being rather aversive (mean $=32.5$, s.d. $=7.4$; $0=$ "very aversive" and $100=$ "very pleasant"). Both groups showed also comparable scores on VAS3 and VAS4: In contrast to what was expected, a 2 (groups) $\times 2$ (slide type) ANOVA did not yield a significant group $\times$ slide interaction, $\mathrm{F}(1,40)<1$. In other words, the experimental procedure was not successful in differentially affecting the affective valence of the CS + and the CS-. Finally, analysis of the VAS5 data showed that a majority of subjects in both groups were aware of the fact that the tone was linked to one of the slides. More than $70 \%$ of the subjects reported a score below 5.0 (VAS5 ranged from $0=$ "very sure that one of the slides was more often followed by a tone" to $10=$ "very sure that both slides were equally often followed by a tone") and only two subjects reported to be absolutely sure that there was no CS+/UCS relationship. The mean of VAS5 was 2.9 for the control and 4.6 for the experimental group, $\mathrm{t}(40)=1.8, \mathrm{p}>.05$.

To explore further the validity of the experimental manipulation, for both 
groups Pearson's p-m correlations were computed between VAS1 (blushing frequency), the differential SCR on the tones (before and after the inflation phase), and the a posteriori reported affective valence of the CS+ and the CSslides (see Tables 5.3a and 5.3b). As can be seen in Table 5.3a, there was a significant correlation between blushing frequency and the affective valence of the CS+ slides for the subjects in the inflation group. This positive correlation indicates that subjects reporting to blush relatively often (BLUSH closer to 0 ) evaluated the CS+ (i.e., the stimulus that was associated with "blushing") as being more negative (CS + closer to 0 ) than subjects who reported to blush less frequently. In addition, a significant correlation was found between blushing frequency and the impact of the inflation procedure as indexed by differential responding during the first phase and the third phase tone. In other words, the correlation between "blush" and "d[tone]" suggests that the more the subjects suffer from blushing in daily life, the better the experimental procedure succeeded. As expected, in the control group none of the correlations reached significance (see Table 5.3b). Thus, taken together, the correlational analysis provide additional support for the validity of the experimental manipulation.

Table 5.3a: Correlation matrix for the inflation group $(n=2 I)$ between blushing frequency (BLUSH) the reported affective valence of the CS+ (CS+) and the CS- (CS), and the difference between the SCR on the first tone during acquisition and the final "test" tone (d/tone]).

\begin{tabular}{lccc}
\hline & CS + & CS- & d[tone] \\
\hline BLUSH & $.49^{*}$ & .14 & $-.47^{*}$ \\
CS + & & -.03 & -.21 \\
CS- & & & -.01 \\
\hline
\end{tabular}

${ }^{*} \mathrm{Q}<.05$, two-railed

Table 5.3b: Correlation matrix for the control group $(n=2 I)$ between blushing frequency (BLUSH) the reported affective valence of the CS+ (CS+) and the CS- (CS), and the difference berween the SCR on the first tone during acquisition and the final "rest" tone (d/tone]).

\begin{tabular}{lccc}
\hline & CS + & CS - & d[tone] \\
\hline BLUSH & -.19 & -.26 & .14 \\
CS + & & .21 & .17 \\
CS- & & & .07 \\
\hline
\end{tabular}




\section{Discussion}

In the present experiment, mild non-threatening tone UCSs were paired to one of two erotic slides that served as CSs. Following the acquisition period, subjects in the inflation group were told that the tone signalled "blushing". It was anticipated that this inflation procedure would result in larger SCRs to subsequent CS + trials and in a relatively negative evaluation of the CS+ slide.

The major results can be summarized as follows: (a) Due to the inflation information, SCRs were larger during the extinction trials than during the acquisition phase; (b) Consistent with the hypothesis, only subjects in the inflation condition showed larger SCRs on the CS + trials than on the CStrials during the extinction phase. Yet, this differential responding was not significantly different between both groups; (c) In line with the hypothesis, only within the inflation group differential responding on CS + and CS- trials was larger during the extinction phase than during the acquisition phase (i.e., a phase $x$ stimulus interaction). However, again, the interaction did not significantly differ between both groups; (d) In contrast to what was expected, subjects' appreciation of the CS + was not differentially affected by the inflation manipulation.

The hypothesis that one instance of UCS inflation can modify conditioned responding in humans is sustained by the finding that only subjects within the inflation condition showed larger SCRs on CS + trials than on CS- trials. The present finding that only in the inflation group differential responding to CS+ and CS- slides was stronger during extinction than during acquisition, provides additional supportive evidence. Yet, neither of these results differed significantly between groups. Therefore, these results do not allow to firmly conclude that the experimental procedure successfully inflated conditioned responding.

Meanwhile, the present inflation manipulation did succeed in inducing a general increase in electrodermal responding irrespective of slide type. The apparent generalisation across stimuli might be attributed to the similarity of the CS + and the CS- slides. It is possible that "inflation" subjects became afraid to blush during CS- trials. Nevertheless, it should be stressed that the inflation procedure had also a specific effect, such that within the inflation group, SCRs increased more on CS + than on CS- extinction trials. In sum, then, the SCR results tentatively suggest that one instance of UCS revaluation may affect conditioned responding, provided that the revaluation procedure is sufficiently salient.

As in Experiment 2, no evidence was found to suggest that the a posteriori reported affective valence of the CS + slide was affected by the UCS inflation procedure. The failure to find an effect in the subjective modality is possibly due to the fact that affective valence was measured after the experiment proper. It might well be that the revaluation effect on affective valence is a time limited phenomenon; that is, the six non-reinforced CS + presentations (after the inflation procedure) may have extinguished differential appreciation 
of CS+ and CS- slides. Thus, stronger revaluation procedures and/or on-line slide evaluations might be necessary to detect revaluation effects on affective valence.

Apart from the above mentioned results that are directly related to the experimental hypotheses, some additional findings emerged that are pertinent to the validity of the "blushing" paradigm. First, subjects in both groups reported that they considered social blushing as being fairly aversive. Thus, the inflated tone may, indeed, be considered as an aversive UCS. In support of this conclusion, SCRs to the tone during the test phase (i.e. after the inflation information had been given) were significantly larger than those during the acquisition phase. Further support for the validity of the revaluation procedure comes from the significant correlation that emerged between "blushing frequency" and the impact of the inflation procedure, as indexed by differential SCRs on acquisition and test phase tones. That is, the more subjects reported to blush in daily life, the better the inflation manipulation succeeded.

Another encouraging finding was that in the inflation group, a correlation emerged between blushing frequency and the subjective valence of the CS+ slide. This result indicates that subjects who report to blush relatively often evaluated the CS + slide as being more negative than subjects who reported to blush less frequently. Thus, this finding adds to the suggestion that the more subjects suffer from blushing in daily life, the larger is the impact of the inflation procedure.

In sum, the present "blush" paradigm appears to be a valid experimental set up to investigate UCS revaluation phenomena in the context of human aversive conditioning. The present findings provided some preliminary evidence to suggest that one instance of UCS revaluation is sufficient to affect conditioned electrodermal responding. However, it should be stressed that the inflation effects found in the present study were too weak to draw any firm conclusions. Moreover, the experimental effects were restricted to the domain of physiological responding. To investigate the reliability of the present UCS inflation effects, a final experiment was performed employing a slightly modified design that aimed at increasing the aversiveness of the inflated UCS.

\section{Experiment 4}

This experiment was conducted to investigate the robustness of the one-trial UCS revaluation effects found in Experiment 3 . The procedure was essentially the same as that used in the former study, with the exception that in the present study two observers were in the experimental room during the experiment proper. This was done in an attempt to increase the aversiveness of the "blushing information" provided at phase 2 . It seems reasonable to argue that blushing is especially aversive if one is the attentional focus of others (e.g., Leary, Britt, Cutlip, \& Templeton, 1992). For the same reason, subjects in this study were all men, as post hoc analyses of the data of experi- 
ment 3 revealed that, in general, men considered blushing as being more aversive than women. Intensifying the UCS inflation procedure was expected to result in stronger UCS inflation effects.

\section{Method}

\section{Subjects}

The subjects were 33 undergraduate men who did not participate in earlier experiments at our department. Mean age was 19 years (range: 18 to 22 years).

\section{Apparatus and Stimulus Material}

All apparatus, stimulus material, and measurements were identical to that described in Experiment 3.

\section{Design}

The design of Experiment 4 was identical to that of Experiment 3. The same held true for the data-analytic strategy that was employed.

\section{Procedure}

Most procedural details were identical to those in Experiment 3 except that one male and one female "observer" were in the experimental room during the experiment proper. The observers were seated between the screen and the subject. One observer was positioned left and the other was positioned right of the screen. Both observers were facing the subject. Observers as well as subjects were explicitly instructed not to communicate with each other. To enhance the credibility of the experimental set-up, the observers made notes during the experiment.

\section{Results}

\section{SCRs}

The left panels of Figure 5.6 depict SCRs to CS + and CS- slides during the acquisition phase. A MANOVA revealed no multivariate effect of conditioning, $\mathrm{E}(2,30)<1$. Univariate $\underline{\mathrm{F}}$ tests showed that neither the mean trend nor the linear trend attained significance, $\mathrm{F}(1,31)=1.31, \mathrm{p}>.20$ and $\mathrm{E}(1,31)<1$, 
respectively. There were no differences between groups in this respect, all $\underline{F} s<1$. Thus, in neither group overt signs of condioned responding emerged.

The right panels of Figure 5.6 show SCRs to CS + and CS- slides during extinction. A MANOVA showed a multivariate effect of conditioning, $\underline{F}(2,30)=3.6 \quad p<.05$. Univariate $\underline{F}$ tests revealed a marginally significant mean trend $[\mathrm{E}(1,31)=3.7, \mathrm{p}=.06]$ as well as a significant linear trend $[\mathrm{F}(1,31)=6.2, \mathrm{p}<.05]$, indicating that responses on $\mathrm{CS}+$ trials were larger than those on CS- trials and that this differential responding declined over trials. No differences between groups emerged in this respect, all $\underline{F}<1$.
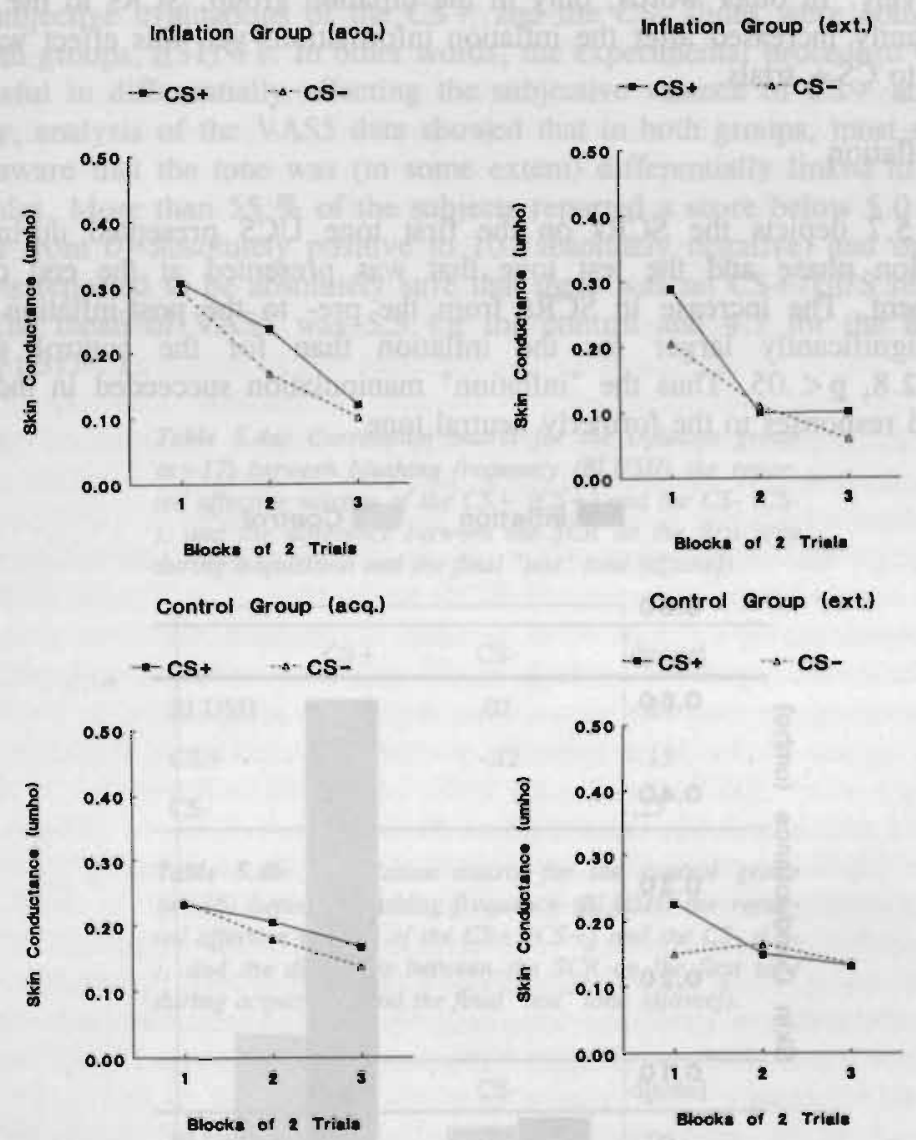

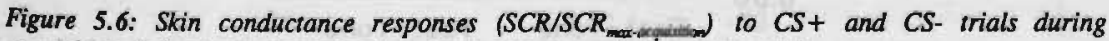
acquisition (lefi panel) and extinction (right panel) in blocks of two trials.

As in Experiment 3, difference scores were computed between SCRs to the last two acquisition trials and those to the first two extinction trials (for both CS + and CS- trials). These scores were subjected to a trend analysis. Mean 
and linear trends were inspected to test phase and phase $\mathrm{x}$ stimulus interaction effects, respectively. A group factor was included to test whether the pattern of results was affected by the experimental manipulation. The univariate $\underline{F}$ tests indicated that neither the mean trend nor the linear trend significantly differed between groups, $\mathrm{F}(1,31)=1.7, \underline{p}=.20$ and $\underline{F}(1,31)<1$, respectively. Yet, within-group analyses revealed a significant mean trend for the inflation group $[\mathrm{E}(1,31)=10.1, \mathrm{p}<.01]$, but not for the control group $[\mathrm{F}(1,31)=1.1$, p>.30]. Neither for the inflation group, nor for the control group, the linear trends attained significance, $\underline{F}(1,31)=1.1, p=.30$ and $\underline{F}(1,31)=2.6, p=.12$, respectively. In other words, only in the inflation group, SCRs to the slides significantly increased after the inflation information, yet, this effect was not limited to CS + trials.

\section{UCS Inflation}

Figure 5.7 depicts the SCRs on the first tone UCS presented during the acquisition phase and the test tone that was presented at the end of the experiment. The increase in SCRs from the pre- to the post-inflation stage were significantly larger for the inflation than for the control group, $\mathrm{t}(28)^{3}=2.8, \mathrm{p}<.05$. Thus the "inflation" manipulation succeeded in inducing enlarged responses to the formerly neutral tone.

Inflation Control

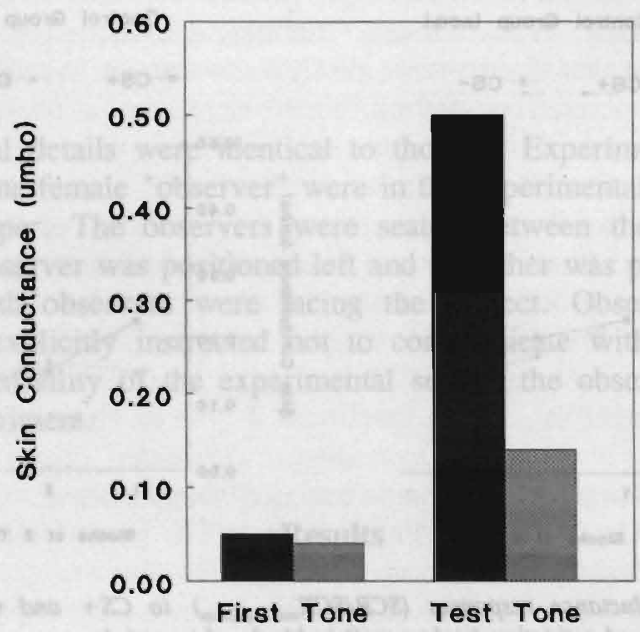

Figure 5.7: Mean SCR to the first tone UCS presented during acquisition and the mean SCR to the test tone presented at the end of the experiment for both the inflation and the control group.

${ }^{3}$ Due to apparatus failure for three subjects SCRs to the test tone rae not available. 
The questionnaire data revealed that both groups did not differ with regard to their "blushing history". That is, VAS1 and VAS2 were similar for both groups, means being 5.0 (control group) and 6.1 (inflation group) for VAS1 $[\mathrm{t}(31)=1.36, \mathrm{p}>.10]$ and 3.0 (control group) and 4.0 (inflation group) for VAS2 $[\mathrm{t}(31)=1.51, \mathrm{p}>.10]$. In addition, the VAS2 data confirmed that subjects considered social blushing as being rather aversive (mean $=3.5$; $0=$ "very aversive" and $10=$ "very pleasant"). In contrast to what was anticipated, subjective evaluations of the CS + and the CS- slides were comparable for both groups, $\mathrm{t}(31)<1$. In other words, the experimental procedure was not successful in differentially affecting the subjective valence of $\mathrm{CS}+$ and $\mathrm{CS}$ Finally, analysis of the VAS5 data showed that in both groups, most subjects were aware that the tone was (to some extent) differentially linked to one of the slides. More than $55 \%$ of the subjects reported a score below 5.0 (VAS5 ranged from $0=$ absolutely positive to $10=$ absolutely negative) and only two subjects reported to be absolutely sure that there was no CS+/UCS relationship. The mean of VAS5 was 5.5 for the control and 4.7 for the inflation group, $\mathrm{t}(31)<1$.

Table 5.4a: Correlation matrix for the inflation group $(n=17)$ between blushing frequency (BLUSH) the reported affective valence of the CS+ (CS+) and the CS- (CS), and the difference between the $S C R$ on the first tone during acquisition and the final "test" tone (d[tone]).

\begin{tabular}{lccc}
\hline & CS + & CS- & d[tone] \\
\hline BLUSH & .35 & .02 & -.20 \\
CS + & & -.12 & -.13 \\
CS- & & & .25 \\
\hline
\end{tabular}

Table 5.4b: Correlation matrix for the control group $(n=16)$ between blushing frequency (BLUSH) the reported affective valence of the CS+ (CS+) and the CS- (CS). and the difference between the SCR on the first tone during acquisition and the final "test" tone (d/tone]).

\begin{tabular}{lccc}
\hline & CS + & CS- & d[tone] \\
\hline BLUSH & -.06 & .22 & -.06 \\
CS + & & -.32 & -.22 \\
CS- & & & -.03 \\
\hline
\end{tabular}

Pearson p-m correlations were computed between VAS1 (blush frequency), the differential SCR to the tones (before and after UCS inflation), and the a posteriori reported affective valence of the CS + and the CS- slides. Although 
the pattern of correlations was similar to that found in Experiment 3, none of the correlations reached significance (see Tables 5.4a and 5.4b).

\section{Discussion}

The following main findings were revealed: (a) During the extinction phase, SCRs to CS + slides were larger than those to CS- slides. However, this differential responding was not especially prominent in the inflation group, (b) Only within the inflation group, SCRs significantly increased after the inflation procedure. Yet, this effect was not limited to CS + trials, neither was this response pattern significantly different from that of the control group, (c) Subjects evaluations of the CS + and the CS- slides were not found to be affected by the inflation procedure.

Taken together, the presents results can be considered as a failure to replicate the promising findings of Experiment 3. Although the pattern of results was quite similar to that of Experiment 3, neither of the relevant between-groups comparisons reached significance. In addition, as in Experiments 2 and 3, no evidence could be obtained that the inflation procedure can change subjects' evaluations of the CS + . In fact, neither at the physiological nor at the subjective level, an experimental effect could be detected. Thus, the hypothesis that one instance of UCS inflation is sufficient to affect human conditioned responding is not supported by the present results.

Although the inflation procedure failed to strengthen conditioned responding, it succeeded in increasing the SCRs to the tone UCS. That is, SCRs to a test presentation of the tone after inflation information had been given were differentially enlarged for subjects in the inflation condition, suggesting that the experimental manipulation was successful. In addition, as in Experiment 3 , the impact of the UCS inflation procedure was related to the subjects' blushing history: The more subjects in the inflation group reported to blush in daily life, the larger was the difference between their SCRs to the acquisition and test phase tones. Furthermore, there was, again, a relationship between subjects' blushing history and the appreciation of the CS+ in the inflation group: Subjects who reported to blush relatively often evaluated the CS+ slides as being more negative than subjects who reported to blush less frequently. Although it should be added that none of the correlations reached the conventional levels of significance, the pattern of results was highly similar to that of Experiment 3.

Meanwhile, no evidence was revealed to suggest that the inclusion of observers in the present paradigm increased the aversiveness of the inflated UCS. The manipulation checks (VAS data) yielded less pronounced results than that of Experiment 3, suggesting, if anything, that the inflated UCS was less aversive in the presence of the observers. If this is, indeed, the case, it would be one explanation for the absence of an effect of inflation on conditioning in the present study.

In sum, then, the present results seem to add to the evidence obtained in 
Experiment 3, that the "blush" paradigm is a valid experimi:ital set-up to test the impact of UCS inflation on human aversive conditioning. Nevertheless, the current study did not reveal any data to confirm the idea that human conditioned responding is susceptible to UCS inflation. Thus, the UCS inflation phenomenon in humans seems to be, at best, a fragile effect.

\section{General Discussion}

Recently, Davey (1989) elaborated a new human conditioning model of acquired fears. According to this model, the CS elicits a representation of the UCS, which, in turn, evokes the conditioned response. Postconditioning factors that influence this UCS representation (i.e., that lead to UCS revaluation) necessarily also affect the strength of the conditioned reaction. The most pertinent implication of this view is, that given an acquired CS-UCS association, further non-associative events (e.g., UCS inflation) can profoundly affect the conditioned fear response.

One attractive aspect of this model is that it can account for two findings that are serious drawbacks for traditional conditioning models of phobia. That is, interindividual differences with regard to non-associative events (i.e., inflation events) may explain why (1) a large part of subjects suffering from phobias can not recall a conditioning experience (e.g. Merckelbach et al., 1989) and (2) a substantial number of non-phobics do recall conditioning events but do not develop a phobia (e.g., Merckelbach, Arrindell, Arntz, \& de Jong, 1992).

Another positive feature of the model is that it nicely fits with the cognitive approach of anxiety disorders (e.g, Clark, 1988). For instance, isolated events that inflate a UCS may be considered as a trigger for catastrophical expectations regarding $\mathrm{CS}+\mathrm{s}$ and, in a similar vein, cognitive therapy can be conceptualised as an attempt to devaluate a formerly inflated UCS.

Although a series of studies have demonstrated that postconditioning increase of the UCS intensity can strengthen conditioned reponding in animals (e.g., Bouton, 1984; Hendersen, 1985; Rescorla, 1974), evidence that the same holds true for human subjects is scarce. To the best of the authors' knowledge, only White and Davey (1989) have examined postconditioning UCS inflation in human subjects. In their study, subjects saw two neutral CSs (triangle/kitchen tap) of which one was repeatedly paired with a mild tone UCS. Next, half of the subjects (inflation group; $n=10$ ) heard 12 successive unsignalled tone UCSs that gradually became stronger. The control group was confronted with UCSs that were kept constant at a low intensity level. During the final extinction phase, all subjects were given test presentations of the CSs. Subjects in the inflation group but not subjects in the control group exhibited a conditioned SCR to the CS that was previously paired with the tone UCS.

Given the potential clinical relevance of the UCS-inflation phenomenon, a series of four laboratory experiments were carried out to cross-validate and 
extend the previous findings of White and Davey (1989). The first experiment was fairly similar to that of White and Davey, except that electric shocks were used as UCS rather than tones. In addition, conditioned responding was not only indexed by SCRs but also by subjects' on-line UCS expectancies. This was done to explore whether the UCS-inflation phenomenon would also occur in the subjective modality. In line with the results of White and Davey, Experiment 1 revealed that the presentation of four gradually increasing shock UCSs significantly retarded the extinction of conditioned responses for both the SCRs and the UCS expectancies.

Although experiment 1 confirmed the earlier findings that conditioned responding in humans can be affected by an UCS inflation procedure, it is still a good step from such procedure to fear acquisition scenarios as they may occur in real life. Note, for instance, that in both experiments neutral (e.g., triangles/flowers) rather than phobogenic (e.g., angry faces or spiders) stimuli were used as CSs. In addition, UCSs were inflated in several steps. Yet, the reconstructed UCS-inflation scenarios of the clinical cases described by Davey et al. (1993) are characterized by more sudden, isolated instances of UCS inflation. Furthermore, thus far only physiological responses and UCS expectancies were measured as an index of acquired fear. Given the multidimensionality of fear, it would be important to know to what extent the affective valence of the CSs is affected by the inflation procedure.

To facilitate extrapolation from experimental findings to clinical cases, Experiment 2 was designed to improve the 'ecological validity' of the inflation paradigm. Phobogenic rather than neutral slides were used as CSs. In addition, only one inflated shock UCS was administered during the inflation period instead of a series of gradually increasing UCSs. Finally, the impact of the inflation procedure on subjects' physiological reactions as well as on their appreciation of the CSs was investigated. However, no differential extinction pattern between the inflation and the control group could be demonstrated in Experiment 2. Moreover, the affective valence of the CS+ slides was not affected by the experimental procedure. One explanation for these null findings could be that one instance of UCS revaluation only induces conditioned responding if the inflation procedure is highly salient and/or of personal concern. A theoretically less attractive explanation for the absence of a UCS inflation effect in Experiment 2 might be that in humans this phenomenon is restricted to a very specific and rather artificial laboratory manipulation. Such an explanation would, of course, imply that UCS inflation is of little relevance to human conditioning models of acquired fear.

To test the former explanation, Experiment 3 was conducted. This experiment aimed at increasing the saliency of the UCS inflation procedure. In this experiment mild, tone UCSs were paired to one of two erotic slides that served as CSs. Following the acquisition period, only subjects in the inflation group were told that the tone signalled "blushing". It was anticipated that this inflation information would result in larger SCRs to subsequent CS + trials and in a relatively negative evaluation of the CS + slide. Consistent with the hypothesis, only subjects in the inflation condition showed larger SCRs on 
CS + trials than on CS- trials during the extinction phase. Yet, this differential responding was not significantly different between both groups. In addition, only within the inflation group, differential responding on CS + and CS- trials was larger during the extinction than during the acquisition trials (i.e., a phase $x$ stimulus interaction). Again, however, the interaction did not significantly differ between both groups. Finally, in contrast to what was expected, subjects' appreciation of the CS + was not differentially affected by the inflation manipulation.

Taken together, the findings of Experiment 3 provide some preliminary evidence to suggest that under certain circumstances, one instance of UCS revaluation is sufficient to induce conditioned responding. One reason for the absence of strong inflation effects in this study could be that the experimental manipulation was not effective enough. However, several findings pertinent to the validity of the "blush" paradigm suggested that the experimental manipulation was credible and succeeded in increasing the aversiveness of the UCS. First, subjects of both groups confirmed that they considered social blushing as being rather aversive. Thus, the inflated tone may, indeed, be considered as an aversive UCS. Second, in line with this conclusion, during the test stage (i.e., after the inflation information had been provided), SCRs to the tone were significantly larger than those during the acquisition stage. Third, the more subjects reported to suffer from blushing in daily life, the better the experimental manipulation succeeded (as indexed by differential responding on first and third stage tones and subjective evaluations of the $\mathrm{CS}+$ ).

Eventhough the "blush" manipulation appears to give rise to UCS inflation, it can not be excluded that the inflated UCS was just not of sufficient intensity to reliably elicit conditioned responding. To investigate this possibility the inflation procedure of the final experiment was slightly modified to increase its aversiveness. Providing that blushing is already aversive if one merely suspects being observed (e.g., as in Experiment 3), it seems reasonable to argue that blushing is even more aversive in case one is actually observed. Thus, to increase the aversiveness of the UCS in Experiment 4, two observers were in the experimental room during slide presentation. Although there was no evidence that the presence of the observers did, indeed, increase the aversiveness of the inflated UCS, the manipulation checks essentially revealed the same positive results as in Experiment 3. Nevertheless, neither at the physiological nor at the subjective level any pertinent experimental effect of UCS inflation could be detected. Thus, the notion that one instance of UCS inflation is sufficient to affect human conditioned responding is down played by the results of Experiment 4.

In sum, then, the present findings seem to converge to the conclusion that to the extent that the inflation procedure gains in "ecological validity", the procedure loses its power to induce conditioned responding. In other words, it appears that human conditioning is susceptible to UCS inflation but only so under very specific (i.e., laboratory) conditions. Apart from the failure to find unequivocal empirical support for UCS inflation in human fear conditioning, there is a more fundamental point that can be raised when one attempts to 
interpret real life phobias in terms of UCS inflation. As Rachman (1991) recently pointed out, neo-conditioning notions such as UCS inflation "are still too liberal" (p. 169; see also van den Hout \& Merckelbach, 1991). As there is no clear definition of what qualifies as a UCS inflation event, one runs the risk of becoming involved in a circular argument: A UCS inflation scenario is advanced to account for the etiology of a phobia, while at the same time this approach is legitimized by the mere presence of that phobia.

One could, of course, counter that a UCS inflation interpretation is warranted by the occurrence of isolated, stressful events in the learning history of anxiety disordered patients. But, then, alternative views make the same predictions under these circumstances. For example, according to Jacobs and Nadel (1985), exposure to stressful conditions may lead to the recovery of fears that were conditioned in childhood and subsequently extinguished. These authors speculated that a stress-induced dysfunction of the inhibitory hippocampus would lead to the re-emergence of previously conditioned fears. Thus, the occurrence of stressful experiences in the learning history of phobic patients does not automatically imply the operation of a UCS inflation process. Meanwhile, there is no independent way (i.e., independent from stressful experiences and the phobic complaints) for establishing the presence of such an inflation process.

\section{Conclusion}

It is tempting to consider UCS inflation as an integral part of human fear conditioning. It elegantly circumvents several problems of more traditional conditioning accounts of phobic fear, thereby avoiding the seemingly artificial distinction between behaviorists' and cognitivists' approaches of fear. Additionally, it is sometimes possible to interpret the learning history of anxiety disordered patients in terms of an inflation scenario. It should be stressed, however, that such seemingly convincing cases are gratuitous in the first place, as long as there is no solid empirical evidence for inflation phenomena affecting fear conditioning in humans. Thus far, the emprirical basis of UCSinflation in humans is meagre. 


\section{5.b UCS-REVALUATION AND PHOBIA: MODULATING FACTORS}

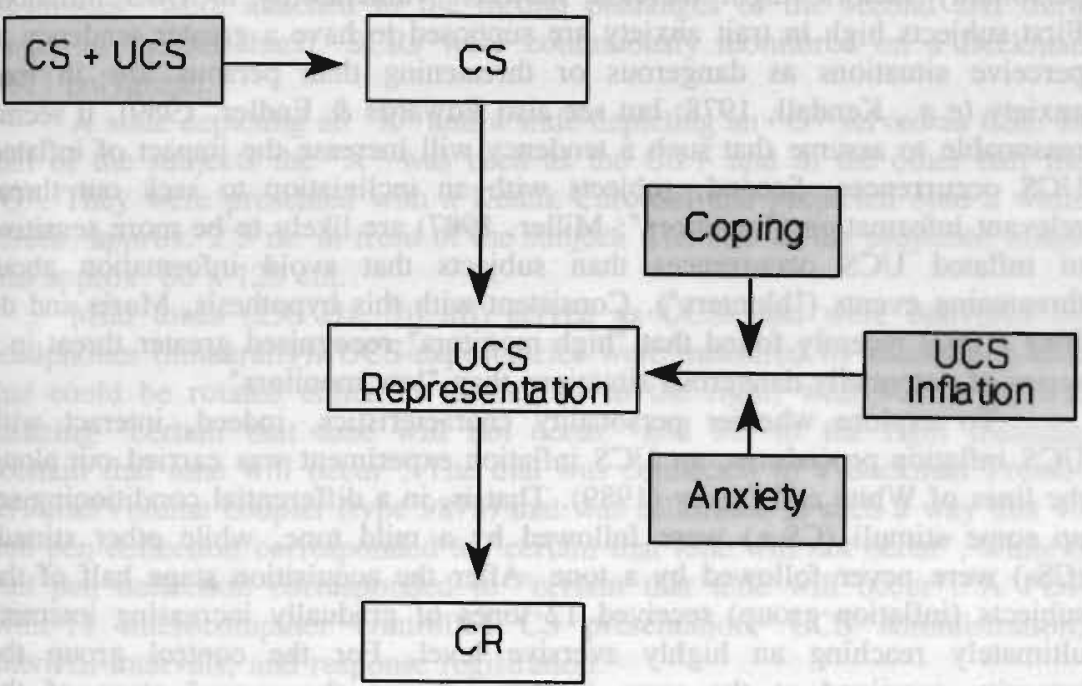

This chapter is partially based on a paper submitted as: De Jong, P.J., \& Muris, P. (1994). UCS inflation, trait anxiety, and monitoring coping style.

Acknowledgements: The authors thank Greetje Koertshuis for her assistance during the dataacquisition. 


\section{A Final Note:}

\section{UCS Inflation, Trait Anxiety, and Monitoring Coping Style}

One factor that might have played a role in the disappointing results presented above, is that individual differences were not taken into account. For instance Eysenck (1976) suggests that a valid theory on human conditioning should incorporate both situational factors and personality characteristics. That is, it might be that subjects' personality characteristics interacted with the experimental UCS inflation scenarios, thereby blurring the results. At least two personality factors might increase subjects' vulnerability to UCS inflation. First subjects high in trait anxiety are supposed to have a greater tendency to perceive situations as dangerous or threatening than persons low in trait anxiety (e.g., Kendall, 1978; but see also Edwards \& Endler, 1989). It seems reasonable to assume that such a tendency will increase the impact of inflated UCS occurrences. Second, subjects with an incliniation to seek out threat relevant information ("monitors"; Miller, 1987) are likely to be more sensitive to inflated UCS occurrences than subjects that avoid information about threatening events ("blunters"). Consistent with this hypothesis, Muris and de Jong (1993) recently found that "high monitors" recognised greater threat in a series of potentially dangerous situations than "low monitors".

To explore whether personality characteristics, indeed, interact with UCS inflation procedures, an UCS inflation experiment was carried out along the lines of White and Davey (1989). That is, in a differential conditioning set up some stimuli $(\mathrm{CS}+)$ were followed by a mild tone, while other stimuli (CS-) were never followed by a tone. After the acquisition stage half of the subjects (inflation group) received 12 tones of gradually increasing intensity ultimately reaching an highly aversive level. For the control group the intensity remained at the same intensity during the second stage of the experiment. During the third stage, subjects were given test presentations of the CS + and the CS-. From the results of White and Davey using an identical experimental procedure, we expected differential SCR responding (i.e., stronger SCRs to CS + slides than to CS- slides) during the test phase, but only for the "inflation group". In addition, a similar pattern of results was expected for the on-line reported probability estimates. Finally, most perinent to the hypothesis under consideration, it was predicted that the experimental effect would even be more pronounced in high monitors and in subjects high in trait anxiety.

\section{Method}

\section{Subjects}

Fifty undergraduate students $(9$ men) participated in the experiment in return 
for a small financial compensation. Their mean age was 21.1 years (range: 17-32).

\section{Apparatus and Stimulus Material}

Skin conductance responses (SCRs) were measured with a Beckman Skin Conductance Coupler (type 9844), using the method of constant voltage $(0.5$ volts). The coupler was connected to two Beckman $\mathrm{Ag}-\mathrm{AgCl}$ electrodes (diameter $8 \mathrm{~mm}$ ) that were filled with isotonic paste following the recommendations of Fowles, Christie, Edelberg, Grings, Lykken, and Venables (1981). Electrodes *were attached to the medial phalanges of the second and third finger of the left hand. SCRs were continuously monitored on a Beckman R611 polygraph.

A slide depicting an " $\mathrm{X}$ " and a slide depicting an "O" served as CSs. In half of the subjects the " $\mathrm{X}$ " was used as the CS + and in the other half the "O". They were presented with a Kodak Carousel and projected onto a white screen, approx. $2.5 \mathrm{~m}$. in front of the subject. The size of the projected image was approx. $80 \times 120 \mathrm{~cm}$.

Mild tones $(250 \mathrm{~Hz}, 70 \mathrm{~dB})$ served as UCSs and were delivered by headphones (binaurally). UCS-expectancies were measured by means of a dial that could be rotated either to the left or to the right, with $90^{\circ}$ to the left meaning "certain that tone will not occur" and $90^{\circ}$ to the right meaning "certain that tone will occur". The dial was connected to a Beckman Pressure/Pulse/Volume coupler (type 9878) that was calibrated in such a way that 40 mm pen deflection corresponded to "certain that tone will not occur", while 0 $\mathrm{mm}$ pen deflection corresponded to "certain that tone will occur". A PDP Minc-11 microcomputer controlled CS presentation, UCS administration, intertrial-intervals, and response registration.

\section{Design}

The experiment was designed according to a 2 (group) $\times 2$ (reinforcement) factorial model. The first between-subject factor refers to the fact that half of the subjects (inflation group, $\mathrm{n}=25$ ) were confronted with a postconditioning inflation of the shock UCS, whereas the other half of the subjects (control group, $n=24$ ) were confronted with postconditioning UCSs that were kept at a constant (low) level. The second within-subject factor pertains to the fact that all subjects saw two slides, one of which $(\mathrm{CS}+$ ) was paired (during acquisition) with a mild UCS, while the other (CS-) was never paired with the UCS.

\section{Procedure}

Before the experiment proper, subjects completed completed two questionnai- 
level which is rather disappointing given the dramatic effect size in SCR responding in a similar study of White and Davey.

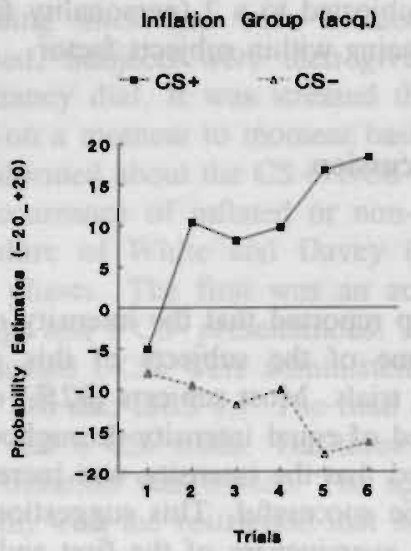

Control Group (acq.)

$-\operatorname{cs}+\quad \operatorname{cs}-$

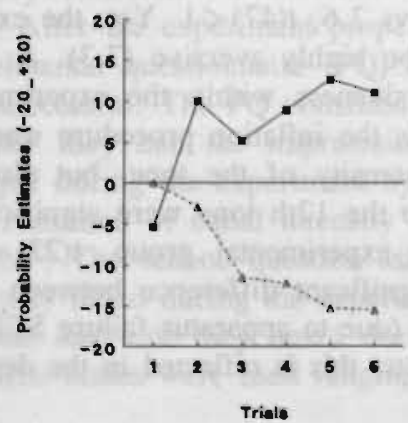

Inflation Group (ext.)

$-\mathrm{CS}+\quad \rightarrow \mathrm{CS}-$

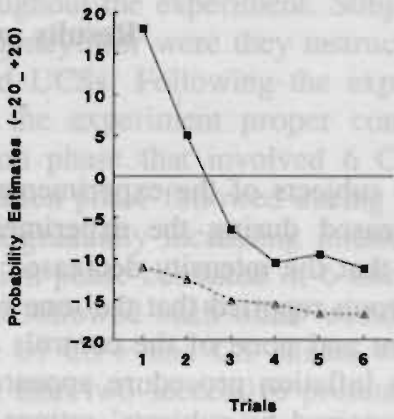

Control Group (ext.)

$-\operatorname{cs}+\quad \rightarrow \operatorname{cs}-$

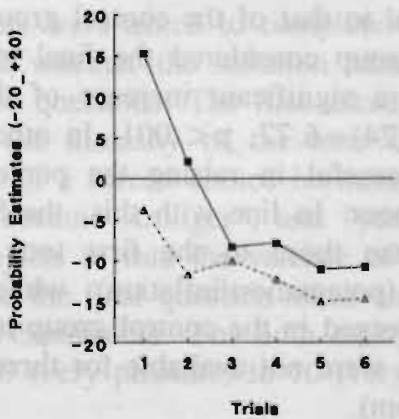

Figure 5.8: On-line shock expectancies during the CS+ and CS-trials for the acquisition stage (left panel) and the "lest" stage (right panel) in blocks of two trials. 


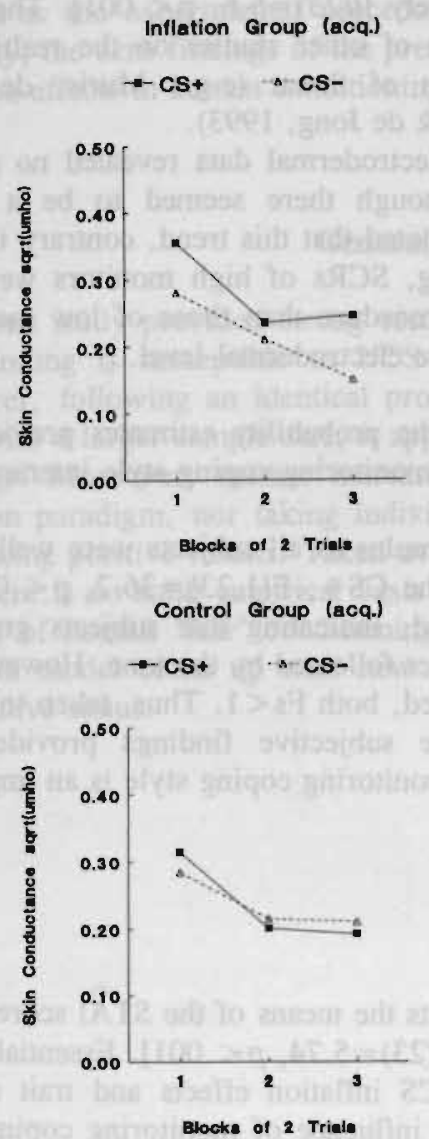

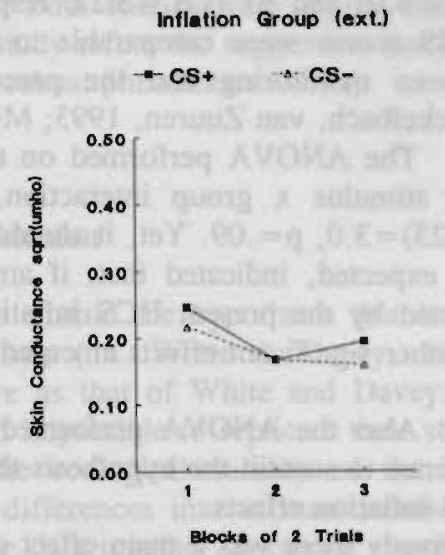

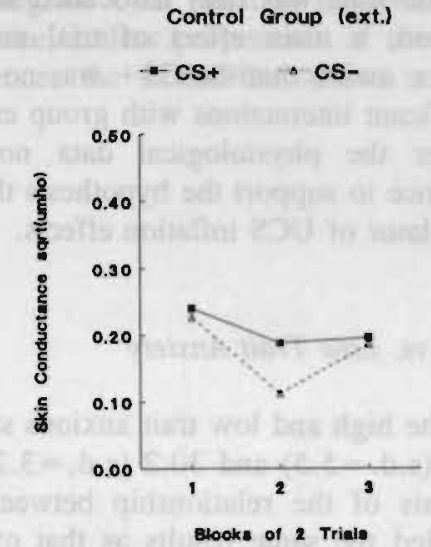

Figure 5.9: Skin conductance responses (SCR/SCR max-and $_{\text {and }}$ ) on $C S+$ and CS- trials during acquisition (left panel) and "extinction" (right panel) in blocks of two trials.

\section{Personality Characteristics and UCS Inflation}

\section{High vs. Low Monitors}

To investigate the influence of a monitoring coping style on the impact of UCS inflation, subjects of the experimental group were divided into high and low monitors according to a median split procedure. Subsequently, data from the test phase were submitted to a 2 (high vs low) $\times 2$ (CS+/CS-) ANOVA, with the last factor being a within subjects factor. If a monitoring coping style is an important modulating factor, a group $\mathrm{x}$ stimulus interaction should emerge.

For the high and the low monitors means of the MBSS were 59 

6.a THE PHENOMENA III: STARTLE MODULATION AND PHOBIA- PART ONE

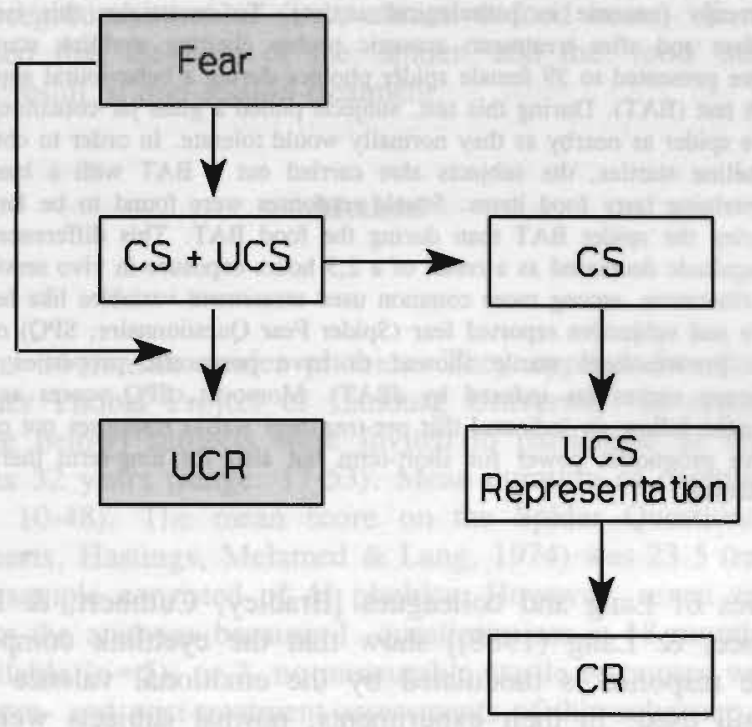

This chapter is based on a paper presented at the First International Congress on Stress, Anxiety. and Emotional Disorders -Braga, 1991. Parts of this paper are published as: de Jong. P.J., Merckelbach, H. \& Arntz, A. (1991). Eyeblink startle responses in spider phobics before and after treatment: A pilot study. Journal of Psychoparhology and Behavioral Assessment, 13, 312223.

Acknowledgerments: The authors wish to thank the therapists Germie van den Berg and Edith Lavy for treating the patients, and Dorien Wolfs for scoring the data. 
Its Evaluative and Prognostic Properties in the Treatment of Spider Phobia.

\begin{abstract}
A number of experiments have shown, that the magnitude of eyeblink startles is directly related to the emotional valence of the foreground stimulus: The magnitude increases linearly from pleasant to unpleasant foreground stimuli. From this, it is suggested that the startle response may provide a useful assessment procedure for therapy prognosis and thereapy outcome in pathological anxiety. To investigate this issue, before and after treatment, acoustic probes eliciting eyeblink startles were presented to 39 female spider phobics during a behavioural approach test (BAT). During this test, subjects pulled a glass jar containing a live spider as nearby as they normally would tolerate. In order to obtain baseline startles, the subjects also carried out a BAT with a basket containing tasty food items. Startle responses were found to be larger during the spider BAT than during the food BAT. This difference in magnitude decreased as a result of a 2,5 hours exposure in vivo session. Furthermore, among more common used assessment variables like heart rate and subjective reported fear (Spider Fear Questionnaire; SPQ) only the pre-treatment startle showed to have prognostic properties for therapy succes (as indexed by dBAT). Moreover, SPQ scores at 18 months follow up indicated that pre-treatment startle responses not only have prognostic power for short-term but also for long-term therapy outcome.
\end{abstract}

Recent studies of Lang and colleagues [Bradley, Cuthbert, \& Lang (1990); Vrana, Spence, \& Lang (1988)] show that the eyeblink component of the startle probe response is modulated by the emotional valence of the foreground stimuli used. In their experiments, normal subjects were confronted with a series of slides depicting pleasant (e.g., nudes of the opposite sex), neutral (e.g., household objects), or unpleasant (e.g., mutilated bodies) stimuli. Acoustic startle probes were delivered during and between slide presentation. Lang and associates repeatedly documented that the startle reflex magnitude increases linearly from pleasant to unpleasant foreground stimuli. This increase in magnitude was found to be independent of arousal and attention attracking properties of the foreground stimuli used. These results led Vrana et al. (1988) to the suggestion that "the startle probe would be useful in evaluating treatment methods and in the prognostic assessment of patients with pathological anxiety" (p.491; see also review by Lang, Bradley \& Cuthbert, 1990).

The present study was designed in order to investigate: 1 . whether acoustic probes, indeed, elicit stronger startle responses in phobic subjects during a fear-relevant foreground stimulus than during a pleasant foreground stimulus; 2 . whether startles elicited in the presence of a fear-relevant foreground stimulus are modulated by behavior therapy; 3 . whether the eyeblink 
startle can be used in predicting and evaluating treatment outcome.

In the current study, startle probes were presented to spider phobics during a behavioral approach test (BAT). During this test, subjects had to pull a glass jar containing a live spider as nearby as they normally would tolerate. In order to obtain baseline values of the startle responses, patients also performed the BAT with a basket containing tasty food items. The food items can be considered as a pleasant foreground stimulus for startle probes. After a one-session exposure in vivo treatment (Ost, 1989) which lasted approximately 2.5 hours, the subjects underwent the same assessment procedure.

On the basis of Vrana et al.'s results (1988), it was predicted that startle responses would be stronger with an unpleasant (i.e., a live spider) than with a pleasant foreground stimulus (i.e., attractive food items). Furthermore, it was anticipated that the ratio of the 'spider' and the 'food' startles would decrease as a result of one-session treatment.

\section{Method}

\section{Subjects}

Subjects were 34 female spider phobics. They applied for therapy at the ongoing Spider Phobia Project of Limburg University. In return of "free" treatment (see below) subjects were invited to participate in research. The mean age was 32 years (range: 17-53). Mean duration of complaints was 23 years (range 10-48). The mean score on the Spider Questionnaire (SPQ; Klorman, Weerts, Hastings, Melamed \& Lang, 1974) was 23.5 (range 20-28). The original sample consisted of 41 phobics. However, seven subjects were excluded from the analyses because 1 . questionnaires at 18 months follow up were not available $(n=5)$; or 2 . no measurable startle responses were obtained $(\mathrm{n}=2)$. Yet, pre- and post-treatment assessments of this subgroup were highly comparable to the remaining subjects.

\section{Assessment and Apparatus}

As a behavioural measure of phobic anxiety, a Behavioural Approach Test (BAT) was used, both before and after treatment. During this test subjects were seated in a chair located behind a large table $(0.3 \mathrm{~m} \times 3.0 \mathrm{~m})$. At the far end of the table, a live spider (Tegenaria atrica) was kept in a closed glass jar. A guided string was fastened to the jar. Subjects were instructed to pull the spider as nearby as they normally would tolerate and to keep the spider in that position for $90 \mathrm{sec}$. It was stressed that by no means should they force themselves. The BAT was scored on a 13-point scale ranging from 0 (spider at $300 \mathrm{~cm}$ ) to 12 (spider on hand). On completion of the BAT, subjects were asked to indicate on $100 \mathrm{~mm}$ Visual Analogue Scales (VASs) their fear during the test. 
When subjects indicated that they had reached their (BAT) limit, startle reflexes were induced by means of $50 \mathrm{~ms}, 90 \mathrm{~dB}$ white noise probes with instantaneous rise time delivered by headphones (binaurally). In total, three probes were given with the first inter-stimulus interval (ISI) being $20 \mathrm{sec}$ and the second ISI being $35 \mathrm{sec}$.

In order to obtain contrast values, subjects carried out a BAT with both a spider and a basket containing tasty food items (e.g., bananas, chocolate, candies, coca-cola, orange juice). Half of the subjects started with the food BAT, while the other half started with the spider BAT. Subjects were randomly assigned to one of the two groups.

Eyeblink startle responses were measured by recording EMG activity from the $m$. orbicularis oculi beneath the left eye, like in the other startle studies. Magnitudes were scored by hand in arbitrary EMG units. During the food and spider BAT, heart rate (HR) was measured by recording pulse volume from the left ear, using a Beckman transilluminated plethysmograph. Physiological signals were fed to a Beckman R 611 polygraph. A microcomputer controled response registration and probe administration.

\section{Procedure}

Upon arrival, subjects were introduced to the laboratory. Following this, subjects completed the SPQ. The SPQ is a 31-item self-report instrument that measures fear of spiders. It has been recommended as a therapy outcome measure (Frederikson, 1983). Next, EMG electrodes and the plethysmograph were attached. After the procedure had been explained, subjects carried out the BATs. Before subjects were treated in the therapy room, electrodes and plethysmograph were removed. The treatment consisted of exposure in vivo accompanied with modeling by the therapist and lasted approximately 150 minutes. The one-session treatment has been found to yield good immediate and long-term results (Öst, 1989; Merckelbach, Arntz \& de Jong, 1991). Following the therapy, EMG electrodes and plethysmograph were reattached and the subjects went through the BATs again. Finally, they completed the post-treatment SPQ. Eighteen months after treatment, subjects were asked to complete a follow-up measurement of the SPQ to obtain long-term treatment results.

\section{Data Reduction and Analysis}

Changes scores of the BAT (0-12) and changes scores of the SPQ were used as outcome measures for short-term therapy succes. Because no behavioural data were available at 18 months follow up, change scores of the SPQ (SPQ before treatment minus SPQ at follow up) were used as outcome measure for long-term therapy succes.

Mean startle magnitudes were subjected to a priori $t$-tests to evaluate 
1. whether the spider startle was stronger than the food startle

(before as well as after treatment); and 2. whether the difference between the spider and food startle decreased as a result of treatment. In other words, whether there was an interaction between foregroud stimulus (spider vs. food) and treatment (before vs. after).

Because there are large differences in EMG responding among individuals, ratios of startle magnitude (i.e., mean magnitude spiderBAT/food$\mathrm{BAT}$ ) were preferred in the regression analyses. Three regression analyses were carried out in order to investigate the prognostic properties of the pretreatment startle respons, SPQ, BAT, subjective reported fear during the BAT (VAS), and HR for treatment outcome as indexed by change scores of the BAT as well as indexed by change scores on the SPQ. In order to control for initial differences, pre-treatment BAT (or SPQ) was included in these analyses.

\section{Results}

\section{Startle responses}

As can be seen in figure 6.1, eyeblink startle responses were larger during the spider BAT than during the food BAT, means being 26.8 and 17.8, respectively $[t(33)=3.70, p=.001]$. After treatment, spider startles were still stronger than food startles, means being 21.7 and 17.9 , respectively $[t(33)=2.67$, $p=.012]$. Yet, the pre-treatment difference between the spider and food startle was significantly larger than the post-treatment difference $[t(33)=2.36$, $p=.024]$, means being 9.0 and 3.9 , respectively. In other words, an interaction emerged between foreground stimulus and treatment. Additional analysis indicated that the decrease of the differential responding was caused by a reduction of the spider startle magnitude $[t(33)=2.17, p=.037]$ rather than an increase of the food startle magnitude $[t(33)=-.03]$.

Closer inspection of the individual data revealed that 12 subjects showed an increase in BAT score (due to treatment) that was not accompanied with a corresponding decrease in differential responding during the spider and food BAT. Post hoc $t$-tests indicated that this subgroup was characterized by significantly smaller pre-treatment differences between the spider and the food startle than the remaining subjects $[t(22.77)=3.97, p=.001$, seperate variance estimate]. These smaller differences between the pre-treatment spider and food startle in this subgroup were caused by larger food startles $[t(14.3)=2.48$, $p=.026$ ] (means being 11.9 and 28.6 ) and not by smaller spider startles $[t(32)=0.02]$ (means being 26.9 and 26.7). Still, the pre- and posttreatment BAT, HR, VAS, and SPQ data were highly comparable for both groups (subgroup vs. remaining subjects; all $t \mathrm{~s}<1.5$, all $p \mathrm{~s}>.2$ ). The strong food startles in this subgroup may be attributed to an overall effect of anxiety induced by the anticipation of the exposure treatment in these subjects. 


\section{Spider \\ Food Items}

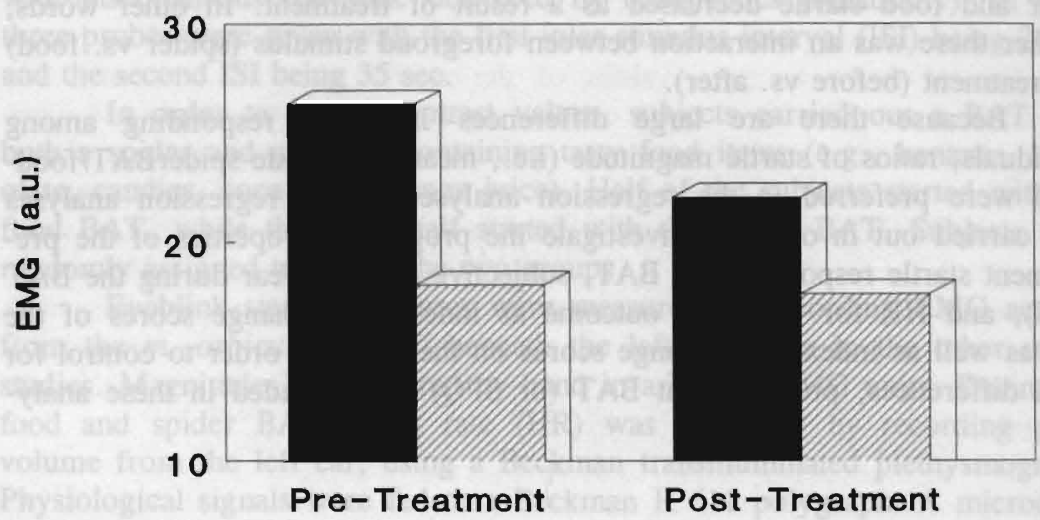

Figure 6.1: Mean magnitudes of the startle responses during the spider BAT and during the food $B A T$, both before and after treatment.

\section{Treatment effects}

In general, the one-session treatment yielded good short-term and long-term results (see table 1). That is, BAT scores increased, SPQ scores decreased, and VAS scores decreased. A notable exception was HR. The cardiac acceleration during the spider BAT was maintained during the post-treatment test. The SPQ scores indicated that therapy succes was maintained at 18 months follow up.

Table 6.1: Mean scores of the behavioral approach test (BAT), the spider questionnaire (SPQ), self reported fear during the BAT (VAS), heart rate acceleration during the BAT (dHR), and the ratios of the startle responses (spider/food BAT) before and after one-session treatment.

\begin{tabular}{lllllll}
\hline phase & pre & post & f.u. & t & df & p \\
\hline BAT $(0-12)$ & 2.9 & 8.9 & - & 12.1 & 33 & .000 \\
VAS (0-100) & 50.9 & 22.3 & - & 5.5 & 33 & .000 \\
dHR & 10.7 & 9.6 & - & 0.72 & 30 & .24 \\
Startle Ratio & 3.4 & 2.0 & - & 1.9 & 33 & .04 \\
SPQ (0-31) & 23.5 & 11.2 & - & 13.8 & 33 & .000 \\
SPQ (0-31) & 23.5 & - & 13.9 & 8.8 & 33 & .000 \\
\hline
\end{tabular}




\section{Pre-treatment startle respons and treatment effects}

Two regression analyses were carried out to investigate the predictive power of the startle response for short-term treatment success. The first regression analysis was performed with the BAT change score being the dependent variable and the pre-treatment startle response ratio, VAS, HR, SPQ, and pretreatment BAT being the predictor variables $(n=31$; due to technical problems, HR data of 3 subjects were not available. In the regression analysis listwise delete was preferred). The results of the regression analysis are summarized in table 2 . The startle ratio appeared to be the only assessment variable with significant prognostic properties for shortterm therapy success. The positive beta value indicates that larger pre-treatment spider startles are associated with larger BAT change scores.

The second regression analysis using SPQ change score as the dependent variable and the pre-treatment startle response ratio, VAS, HR, BAT, and pre-treatment SPQ being predictor variables demonstrated that non of the used variables has predictive power for post-treatment SPQ score. Finally, a third regression analysis was performed to investigate the prognostic properties of the pre-treatment startle response for long-term treatment effect. This analysis was carried out with $S P Q_{\text {pre minus follow up }}$ being the dependent variable and the pre-treatment startle ratio, HR, VAS, BAT, and SPQ being the predictor variables. As can be seen in table 6.3 , the startle ratio has significant predictive power for long-term therapy succes. The larger the relative strength of the spider startle the better is the long-term therapy prognosis. Only the pretreatment SPQ score was a better predictor for therapy success. However, this finding may simply reflect that higher initial SPQ scores being associated with more room to move in a downward direction.

Table 6.2: Results of the regression analysis $(n=31)$ revealing the prognostic properties of the pre-treatment values of $H R, S P Q$. Startle Response Ratio, BAT, and VAS for therapy success las indexed by the change scores of the BAT). The beta value refers to standardized data.

\begin{tabular}{lrrl}
\hline Variable & Beta & t(5.30) & D \\
\hline HR & & & \\
SPQ & 0.24 & 1.55 & .13 \\
Startle Ratio & -0.30 & -1.91 & .07 \\
BAT & 0.40 & 2.75 & .01 \\
VAS & -0.52 & -3.04 & .005 \\
& -0.19 & 3.57 & .28
\end{tabular}

Note: Presented results are obtained from the equation in which all variables were included. 
Table 6.3: Results of the regression analysis $(n=31)$ revealing the prognostic properties of the pre-treatment values of $H R, S P Q$. Startle Response Ratio, BAT, and VAS for therapy success (as indexed $S P Q_{\text {pre }}$ minus $\left.S P Q_{\text {follow up }}\right)$. The beta value refers to standardized data.

\begin{tabular}{lrrl}
\hline Variable & Beta & $\mathfrak{t}(5,25)$ & $\mathrm{Q}$ \\
\hline HR & -0.04 & -0.20 & .84 \\
SPQ & 0.39 & 2.31 & .03 \\
Startle Ratio & 0.36 & 2.11 & .04 \\
BAT & 0.17 & 0.89 & .38 \\
VAS & 0.19 & 0.94 & .36
\end{tabular}

Note: Presented results are obtained from the equation in which all variables were included.

More detailed information concerning the startle ratio in relation to other common used outcome measures is provided in the correlation matrix (see table 4).

Table 6.4: Pearson p-m correlations between the pre-treatment startle ratio, the behavioral approach task (BAT), the spider questionnaire (SPQ), reported fear during the BAT (VAS), and heart rate acceleration during the $B A T$ (dHR).

\begin{tabular}{lllll}
\hline & Startle Ratio & VAS & BAT & SPQ \\
\hline VAS & 0.21 & & & \\
& $(34)$ & & & \\
BAT & 0.06 & $-0.51^{*}$ & & \\
& $(34)$ & $(34)$ & & \\
SPQ & 0.07 & 0.15 & -0.29 & \\
& $(34)$ & $(34)$ & $(34)$ & \\
dHR & 0.00 & $0.47^{*}$ & -0.28 & 0.08 \\
& $(31)$ & $(31)$ & $(31)$ & $(31)$ \\
\hline
\end{tabular}

* $\mathrm{p}<0.005$

\section{Discussion}

Three major conclusions can be drawn from the results presented above. First, acoustic probes elicit stronger startle responses in spider phobics during a fearrelevant (i.e., a live spider) than during a pleasant foreground stimulus (i.e., attractive food items). Thus, the present data are in accordance with the results of recent studies (Vrana et al. , 1988; Bradley et al., 1990) which show that the magnitude of the startle response in normal subjects is potentiated when unpleasant foreground stimuli are used. In line with the theoretical framework of 
Lang et al. (1990), one could argue that the obvious negative emotional valence of a live spider for a spider phobic potentiates the startle response and/or that the positive emotional valence of attractive food items inhibits the startie response. As in the present study no neutral foreground stimulus was employed, the results are not decisive in this respect. However, it seems reasonable to assume that the differential responding during the 'food'BAT and 'spider'BAT is mainly carried by the negative affective response to the spider. This suggestion is sustained by the decrease of the ratio of the 'spider' and the 'food' startles after treatment (see below). It should be stressed that in the present study a considerable difference in relative magnitude could be detected between the startle response during the "spider" BAT and the "food" BAT despite the fact that the patients in general were quite stressed in anticipation of the exposure treatment. Furthermore, it is worth noting that this effect was equally potent whether the 'food'BAT'spider'BAT or the 'spider'BAT-'food'BAT order was used.

Second, as a result of the one-session exposure in vivo the relative (as well as the absolute) magnitude of the startle probe response during the "spider" BAT was reduced. Although a test-retest effect can not be ruled out (as a no-treatment control group was lacking), it seems reasonable to suggest that this decrease was largely brought about by the treatment. This suggestion is sustained by the finding that the magnitude of the "spider" startles was lower after treatment while the "food" startles remained unaffected. The reduction occurred notwithstanding the fact that in general, subjects were closer to the spider after treatment than before treatment. In case the post-treatment probes would have been presented with the spider at the same distance as during the pre-treatment BAT rather than at the point of maximal tolerance, an even greater reduction of the startle response during the "spider" BAT might have occurred. As the BAT prevailed for clinical reasons, the former option was impossible to realize. Nevertheless, the present results demonstrate that even under sub-optimal (i.e., clinical) conditions, a treatment effect on startle responses can be documented. This, of course, stresses the robustness of phenomenon under consideration.

Third, the eyeblink startle of spider phobics appears to be relatively independent of other outcome measures such as SPQ, BAT, and HR.

Taken together, the results of the present study confirm the suggestion made by Vrana et al. (1988) that the startle response might be a fruitful outcome measure. However, not all subjects who benefited from the treatment (as indexed by the dBAT scores) showed a decrease of the startle probe response during the spider BAT. As a matter of fact, 12 out of 34 the subjects showed an increase in BAT scores which was not paralleled by a corresponding decrease in startle probe response. However, this subgroup is characterized by the absence of the expected difference between the pre-treatment food BAT and spider BAT startle responses. Possibly, these subjects' responses to the food were influenced by a negative affect induced by the mere knowledge that they would be exposed to spiders in the near future.

The present data show that the stronger the pre-treatment spider startles the more subjects profit from exposure therapy as indexed by changes scores of the BAT (short-term) as well as indexed by the change scores of the pre-treatment SPQ and the SPQ at 18 months follow up (long-term). The magnitude of the startle response appeared to be not associated with the scores of the SPQ that 
subjects completed immediately after treatment. Yet, it is conceivable that the post-treatment SPQ is less reliable than both $S P Q_{\text {pre-treatment }}$ and $S P Q_{\text {follow up }}$ because subjects did not got the chance to test their new attitude against spiders in real live situations. Therefore, it seems reasonable to argue that before treatment as well at 18 months follow up the SPQ is reflecting more closely subjects real handling with spiders whereas the $\mathrm{SPQ}_{\text {post-trearment }}$ is reflecting more subjects (perhaps incorrect) expectations.

The finding that relatively large startle responses are positively correlated with therapy outcome is in line with earlier studies indicating that physiological responsiveness is related to therapy success. For instance Lang, Melamed, and Hart (1970) showed that phobic subjects who revealed a relatively large heart rate increase during initial feared images improved more as a result of systematic desensitization than relatively weak reactors. Also, physiological reactivity during exposure treatment is found to be related to outcome of treatment in pathological anxiety (e.g., Watson \& Marks, 1971). Foa and Kozak (1986) consider physiological reactivity to fear-relevant stimuli as an indicator of emotional processing. In their conceptual framework, emotional processing of feared stimuli is a prerequisite for phobic fears to extinguish. It might well be that a failure to evoke a relatively large startle during the BAT reflects an avoidance of the information presented. From this perspective, the present data sustain their model. That is, it can be speculated that those subjects with strong pre-treatment startles may have profitted more from corrective information presented during the in vivo exposure than weak reactors. In its turn the exposure to corrective information probably facilitated the extinction of the fear of spiders in the strong reactors.

Taken together, present results show that the startle probe response has prognostic properties for both short-term and long-term (18 months) therapy outcome. The proportion of explained treatment effect in the present population is about $15 \%$. On the one hand, this might seem impressive, particularly given the numerous potentially sources of error in the startle response methodology used here. On the other hand, from a clinical perspective this $15 \%$ is, at best, very modest, especially if one considers that it is difficult to create more favourable conditions in clinical practice. Thus, the present results may be seen as promising for the future use of the startle probe methodology as a prognostic tool. Meanwhile, it would be premature to recommend such methodology as a prognostic tool on basis of the present study. In addition, it should be noted that our subjects suffered from a simple phobia. Further research is needed in order to investigate whether similar effects can be demonstrated in other more complex anxiety disorders.

In sum, the current data demonstrate that spider phobics show relatively greater eyeblink startles in the presence of a spider than in the presence of tasty food items. Furthermore, the present study suggests that this differential responding is affected by treatment. In addition, the results suggest that the startle probe response is a fruitful outcome measure that is independent from commonly used outcome measures. Finally, although this study is far from conclusive regarding the prognostic properties of the startle response, the present findings can be taken as promising. 
6.b THE PHENOMENA III: STARTLE MODULATION AND PHOBIA-PART TWO

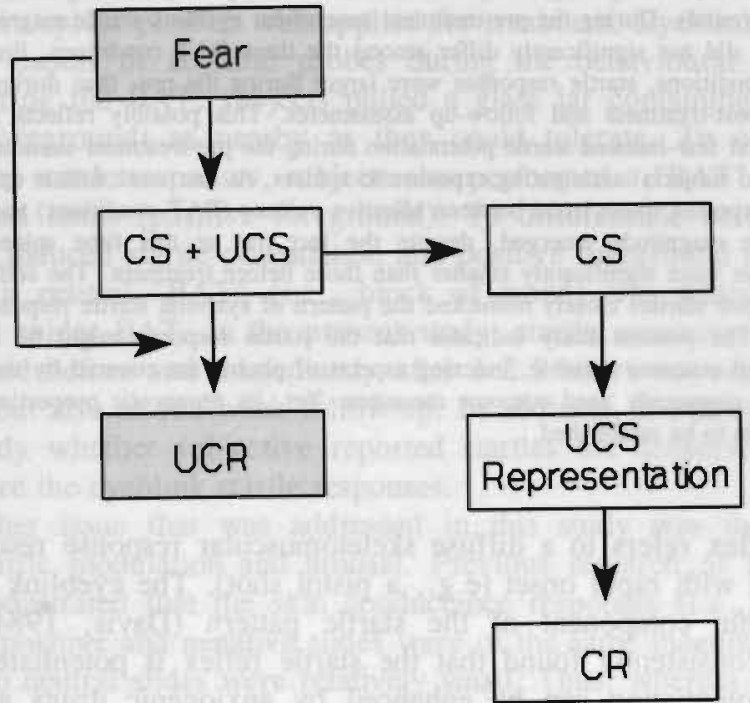

This chapter is partly based on a paper presented at the First European Congress on Psychophysiology (Tilburg. 1991). A less elaborated version of this chapter is published as: De Jong. P.J., Arntz, A., \& Merckelbach, H. (1993). The starte probe response as an instrument for evaluating exposure effects in spider phobia. Advances in Behaviour Research and Therapy, 15, 301-316

Acknowledgements: The author wish to thank the therapists Arie Dijkstra, Edith Lavy. Birgit Maier, and Anja Meijboom for treating the patients, and Dorien Wolfs for taking care of the organisational aspects of this project. Finally we are grateful to Karel Eisses who provided Drosophilae for our spiders. 


\title{
The Startle Probe Response as an Instrument for Evaluating Exposure Effects in Spider Phobia
}

\begin{abstract}
Before treatment, immediately after treatment, and at one week follow up, acoustic probes eliciting eyeblink startles were presented to 37 female spider phobics during a behavioural approach test (BAT). To obtain control startles, the subjects also carried out a BAT with a block of wood (neutral foreground) and a BAT with tasty food items (positive foreground). During the pre-treatment assessment eyeblink startle magnitudes did not significantly differ among the three BAT conditions. For all conditions, startle responses were larger during the pre- than during the post-treatment and follow-up assessments. This possibly reflects a general fear-induced startle potentiation during the pre-treatment session, due to subjects' anticipating exposure to spiders. At one week follow up, the expected linear trend between affective valence (BAT conditions) and startle magnitude emerged, despite the fact that at this time spider startles were significantly smaller than those before treatment. The selfreported startles closely minicked the pattern of eyeblink startle responses. The present study indicates that the startle response might be a fruitful outcome variable, indexing aspects of phobia not covered by the more commonly used outcome measures. Yet, its prognostic properties remain to be established.
\end{abstract}

The startle reflex refers to a diffuse skeletomuscular response resulting from intense stimuli with rapid onset (e.g., a pistol shot). The eyeblink is the first and most stable component of the startle pattern (Davis, 1984). Animal research has consistently found that the startle reflex is potentiated by fear. This startle potentiation can be enhanced by anxiogenic drugs and can be blocked by anxiolytic drugs like diazepam (Davis, Hitchcock, \& Rosen, 1989).

Recent studies of Lang and colleagues (Vrana \& Lang, 1990; Bradley, Cuthbert \& Lang, 1990; Vrana, Spence \& Lang, 1988) show that the human startle probe response is also modulated by emotional state. That is to say, positive foreground stimuli (e.g., slides depicting nudes) appear to inhibit the startle reflex, whereas the startle reflex is potentiated during a negative foreground stimulus (e.g., slides of mutilated bodies). Apparently, a match of the (negative) affective tone of the probe (i.e., a sudden loud noise) with the foreground stimulus leads to a potentiation, whereas a mismatch results in an inhibition of the response. The repeatedly documented linear relationship between affective tone of the foreground stimulus and the startle magnitude, led Vrana et al. (1988) to argue that "the startle probe would be useful in evaluating treatment methods and in the assessment of therapy prognosis of patients with pathological anxiety" (p.491; see also review by Lang, Bradley \& Cuthbert, 1990).

Results of a pilot study tentatively confirmed that the startle probe 
reflex has prognostic and evaluative properties in the treatment of spider phobia (de Jong, Merckelbach \& Arntz, 1991). In that study startle responses were found to be larger during a phobia relevant foreground (i.e., live spider) than during a positive foreground (i.e., basket with food items). In addition, this difference in magnitude decreased as a result of treatment. Furthermore, evidence was found to suggest that the pre-treatment startle responses predict long-term therapy outcome defined in terms of self-reported spider fear at 18 months follow-up (de Jong, Arntz \& Merckelbach, 1991).

Given the potential importance of those findings, the major aim of the present study was to replicate and extend the pilot study. As in the pilot study, subjects were spider phobics who applied for treatment. Eyeblink startles were elicited by means of acoustic probes during the behavioural approach test (BAT). During the BAT, subjects pulled a glass jar containing a live spider (negative foreground) as nearby as they could tolerate. To obtain contrast values of the startle responses, subjects also performed a "BAT" with a basket of tasty food items (positive foreground). To differentiate between negative foreground induced startle potentiation and positive foreground induced startle inhibition, a neutral "BAT" (i.e., block of wood) was added to the food "BAT" and spider BAT. In the present study, startle measurements were not only obtained before and immediately after a 2.5 hours one-session exposure treatment, but also at one week follow-up. In addition, it was explored in the present study whether subjective reported startles are sensitive to the same factors as are the eyeblink startle responses.

Another issue that was addressed in this study was the relationship between startle modulation and arousal. Previous research of Bradley et al. (1991) demonstrated that the skin conductance responses (i.e., physiological arousal) to positive and negative slides were of the same magnitude, while the responses to neutral slides were relatively small. Thus, whereas startle magnitudes show a linear increase from positive to negative slides, the relationship between arousal and slide valence appears to reflect a quadratic (U-shaped) function. In other words, within the context of slide presentations, affectinduced startle modulation appears to be independent of arousal. To investigate whether in the present context the startle reflex is likewise modulated by affect rather than by arousal, heart rate (HR) and self-reported arousal were measured during each BAT.

Given the findings of Bradley et al. (1991) it was anticipated that HR and self-reported arousal would show an U-shaped relationship with the affective valence of the BATs. The startle probe responses were expected to be strongest with a fear evoking foreground stimulus (i.e., spider) and weakest with a pleasant foreground stimulus (i.e., food), while an intermediate magnitude was expected when using the neutral foreground stimulus (i.e., wood). Additionally, it was predicted that the magnitude of the startles during the spider BAT would decrease as a result of treatment. 


\section{Method}

\section{Subjects}

Subjects were 37 female spider phobics. They applied for therapy at the ongoing Spider Phobia Project of Limburg University. In return for "free" treatment (see below), subjects were invited to participate in research. The mean age was 25 years (range: 17-48). The mean score on the Spider Questionnaire (SPQ; Klorman, Weerts, Hastings, Melamed \& Lang, 1974) was 22.1 (range 13-31). The original sample consisted of 42 phobics. However, five subjects were excluded from the analyses because 1 . they did not show up at one week follow up $(n=2)$; or 2 . no measurable startle responses were obtained $(n=3)$.

\section{Assessment and Apparatus}

As a behavioural measure of phobic anxiety, subjects underwent a Behavioural Approach Test (BAT). This was conducted both before and after treatment, and at one week follow up. During this test subjects were seated in a chair located behind a large table $(0.3 \mathrm{~m} \mathrm{x} 3.0 \mathrm{~m})$. At the far end of the table, a live spider (Tegenaria atrica) was kept in a closed glass jar. A guided string was fastened to the jar. Subjects were instructed to pull the spider as nearby as they could tolerate and to keep the spider in that position for $90 \mathrm{sec}$. It was stressed that it was very important to really reach their limits. The BAT was scored on a 13-point scale ranging from 0 (spider at $300 \mathrm{~cm}$ ) to 12 (spider on hand) (see Arntz, Lavy, van den Berg \& van Rijsoort (1993), for more details concerning the BAT). On completion of the BAT, subjects were asked to indicate on $100 \mathrm{~mm}$ Visual Analogue Scales (VASs) their fear during the test $(0=$ no fear, $100=$ terrified).

Five pertinent startles were elicited during the final 90 seconds of each BAT by presenting acoustic probes. The probes consisted of $50 \mathrm{~ms}, 100 \mathrm{~dB}$ white noise probes with instantaneous rise time delivered by headphones (binaurally). The mean inter-stimulus interval (ISI) was $20 \mathrm{sec}$. To familiarize subjects with the acoustic probes, three test probes were delivered while the subjects were pulling the string.

During the pre-treatment spider BAT, probes were given with the spider being in the final position for each subject. To provide the opportunity to evaluate the treatment effect on the startle probe responses, during the posttreatment and follow-up sessions, subjects were instructed to bring the spider in the same position as they did before treatment (this point was marked with a small bar) and to keep the spider in that position for $90 \mathrm{sec}$. Thus, during all three assessments the subjects' distance to the spider was equal.

In order to obtain contrast values, subjects not only carried out BATs with a spider but also with a basket containing tasty food items (e.g., bananas, chocolate, candies, coca-cola, orange juice) and a block of wood. The order 
was balanced. Subjects were randomly assigned to one of the six groups. Thus, one third of the subjects started with the spider BAT, one third started with the food BAT, and one third started with the wood BAT. For each subject the same order was maintained for all sessions. During both the posttreatment and the follow up sessions, subjects ended with the maximal approach task (spider $\mathrm{BAT}_{\max }$ ). The $\mathrm{BAT}_{\max }$ was used as the behavioural index of treatment outcome.

Eyeblink startle responses were measured by recording EMG activity from the musculus orbicularis oculi beneath the left eye, using Beckman miniature $\mathrm{Ag}-\mathrm{AgCl}$ surface electrodes filled with Hewlett Packard Redux creme. The EMG signal was fed to a Beckman EMG Coupler (9852A). Frequencies were filtered $60 \mathrm{~Hz}$ high-pass ( $48 \mathrm{db} /$ octave), using a Krohn Hite filter (type 3341 with a Butterworth characteristic). The EMG signal was then rectified and integrated by a contour following integrator of the type recommended by Fridlund (1979). In order to optimize the sensitivity for momentary fluctuations of EMG activity, a short integration time constant was chosen $(1 / 16 \mathrm{sec})$. The transformed signals were fed to a Beckman polygraph using 3 different channels. To maintain optimal sensitivity for both incubating and habituating responses, each channel used a different amplification factor $(0.5$, 2 , and 5 , respectively). Magnitudes were scored by hand in arbitrary EMG units. During the food, wood, and spider BATs, heart rate (HR) was measured via Beckman $\mathrm{Ag}-\mathrm{AgCl}$ electrodes filled with Hewlett-Packard Redux Creme. The electrodes were placed according to a lead II placement. Electrodes were connected with a Beckman Voltage/Pressure/Volume Coupler (type 9853A). Physiological signals were fed to a Beckman R 611 polygraph. A microcomputer controled response registration and probe administration.

Immediately after each BAT, subjects were asked to report to what extent they were startled by the probes on a VAS ranging from $0 \mathrm{~mm}$ (not startled at all) to $100 \mathrm{~mm}$ (extremely startled). In addition they were asked to rate the foreground stimuli (i.e., spider, wood, and food) on the dimensions of affective valence and arousal by using a VAS.

\section{Procedure}

Upon arrival, subjects were introduced to the laboratory. Following this, subjects completed the SPQ. The SPQ is a 31 -item self-report instrument that measures fear of spiders. It has been recommended as a therapy outcome measure (Fredrikson, 1983). Next, EMG and HR electrodes were attached. After the procedure had been explained, subjects carried out the BATs. Before subjects were treated in the therapy room, electrodes were removed. The treatment consisted of exposure in vivo accompanied by modeling by the therapist and lasted exactly 150 minutes. The one-session treatment has been found to yield good immediate and long-term results (Öst, 1989; Merckelbach, Arntz \& de Jong, 1991; Öst \& Salkovskis, 1992; Arntz \& Lavy, 1993). Immediately after the one-session therapy and at one week follow up, subjects 
went through the BATs again. On both occassions, they also completed a SPQ. Finally, they received a booster session lasting approximately 1,5 hours.

\section{Data Reduction and Analysis}

Changes scores of the BAT (0-12) and changes scores of the SPQ were used as indices for therapy succes.

To check whether the manipulations of the foregrounds (the three BATs) were successful, subjects' judgments of the foreground stimuli along the dimensions of affective valence and arousal were evaluated by using MANOVA trend analyses. A linear trend from spider to food BAT (that should be weakened as a result of treatment) was expected for the affective valence dimension. Reported arousal was expected to be relatively low for the wood BAT in comparison to the food and the spider BAT. In other words, quadratic trends were anticipated.

MANOVA trend analyses were performed on mean eyeblink startle magnitudes and subjective startle responses to evaluate 1 . whether the startles linearly increased from the food via the wood to the spider BAT (before as well as after treatment and at one week follow up); and 2. whether the startle pattern among conditions was modulated by the treatment. Therefore, mean, linear, and quadratic trends were inspected.

Heart rate (HR) data were also subjected to MANOVA trend analyses to test the hypothesis that HR follows (reported) arousal rather than (reported) affect (e.g., Lang et al., 1990), whereas the reverse was expected for the startle response. In other words, a quadratic trend was anticipated due to the relatively weak arousing properties of the wood BAT in comparison to the spider and food BAT.

Four backward regression analyses were carried out in order to investigate the prognostic properties of the pre-treatment startle response, subjective startle, SPQ, BAT, subjective reported fear during the BAT (VAS), and HR for treatment outcome as indexed by change scores of the BAT as well as indexed by change scores on the SPQ. Because there are large differences in EMG responding among individuals, ratios of startle magnitude (i.e., mean magnitude spiderBAT/foodBAT) were preferred in the regression analyses.

Finally, Pearson's p-m correlations were computed to investigate the interrelationship between the pre-treatment subjective and eyeblink startle ratio and other more commonly used outcome measures; namely, he behavioural approach task (BAT), SPQ, and heart rate acceleration during the BAT.

\section{Results}

Due to apparatus failure, the post-treatment startle data of one subject was not available. For the same reason, startle data from the follow-up assessment for two other subjects were missing. Because of technical problems, HR of one 
subject could not be obtained during the follow-up session. This is reflected in the degrees of freedom for the relevant comparisons. In the overall MANOVAs listwise deletion was preferred.

\section{Treatment effects}

In general, the one-session treatment yielded good immediate and short-term results (see Table 6.5). That is, BAT scores increased, SPQ scores decreased, and self-reported fear during the BAT (VAS) decreased. As can be seen in Table 6.5, the treatment effect was maintained at one week follow up.

Table 6.5. Mean scores of the behavioral approach test (BAT), the Spider Phobia Questionnaire (SPQ), self reported fear during the BAT (VAS), before (pre) and after (post) one-session treatment, and at one week follow-up (f.u.).

\begin{tabular}{lcccccc}
\hline PHASE & Pre & Post & F-up & \multicolumn{1}{l}{$t$} & df & $p$ \\
\hline BAT $(0-12)$ & 4.0 & 9.7 & & 13.4 & 36 & $<.01$ \\
BAT $(0-12)$ & 4.0 & & 8.4 & 9.1 & 36 & $<.01$ \\
VAS $(0-100)$ & 61.9 & 35.8 & & -5.2 & 36 & $<.01$ \\
VAS (0-100) & 61.9 & & 52.9 & -1.9 & 36 & .06 \\
SPQ (0-31) & 22.1 & 11.7 & & -11.9 & 36 & $<.01$ \\
SPQ (0-31) & 22.1 & & 13.8 & -9.7 & 36 & $<.01$ \\
\hline
\end{tabular}

\section{Self-report ratings}

Affective valence. Figure 6.2 depicts the self-report ratings of the three different foreground stimuli. As expected, there was a linear trend from spider to food at the pre-treatment assessment, $\underline{F}(1,36)=190.1, \underline{p<.001}$. This pattern of results was maintained at post-treatment and at one week follow-up, $\underline{F}(1,36)=92.5, \mathrm{p}<.001$, and $\mathrm{E}(1,35)=123.2, \mathrm{p}<.001$, respectively. The slope of the linear trend decreased as a result of treatment, $F(1,35)=40.5$, $\mathrm{R}<.001$, and $\underline{F}(1,35)=11.9, \underline{p}<.01$, reflecting the change of affective valence of the spider after therapy.

Arousal. In line with the apriori predictions, there was a quadratic trend of the self-reported arousal from spider to food during all assessments, $F(1,36)$, $\mathrm{p}<.001, \mathrm{~F}(1,36)=46.2, \mathrm{p}<.001$, and $\mathrm{F}(1,35)=44.3, \mathrm{p}<.001$, respectively. The quadratic trend between foreground stimulus and arousal was not affected by treatment, both $\underline{F} s(1,35)<1$. Only during the pre-treatment assessment there was also a significant linear trend, indicating that the pre-treatment spider BAT was more arousing than the pre-treatment food BAT, $\underline{F}(1,36)=8.9, \underline{p}<.005$. 


\section{Spider Wood Wood}

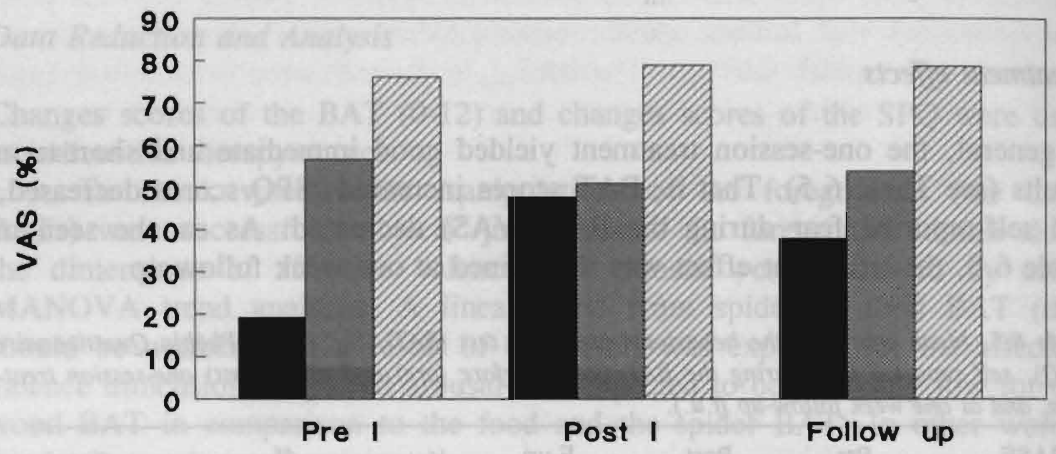

Arousal
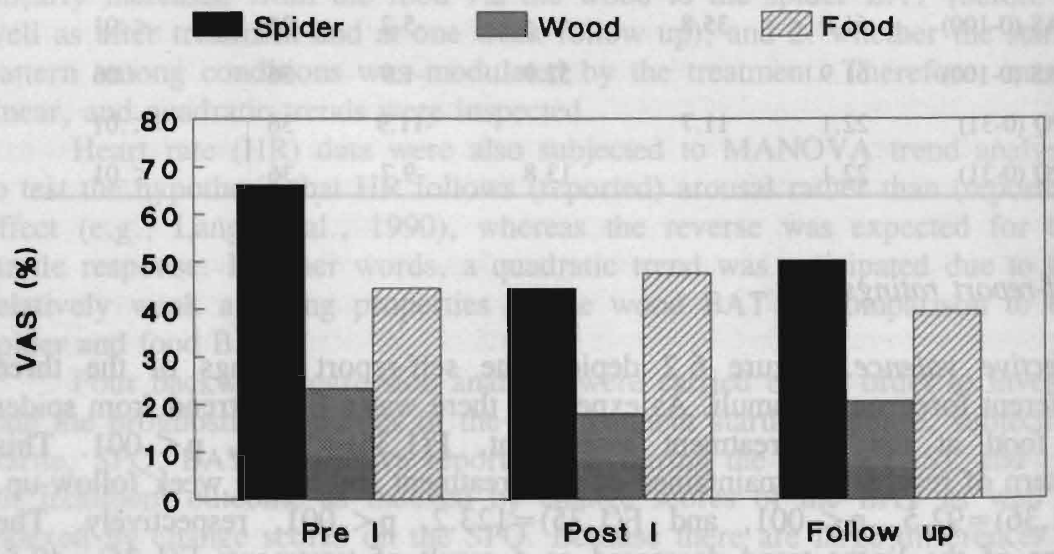

Figure 6.2. Ratings of the foreground stimuli (i.e., spider, wood, food) on the dimensions of affective valence and arousal.

\section{Heart rate}

In line with the hypothesis, pre-treatment HR showed a quadratic trend between the three foreground stimuli, $\mathrm{F}(1,36)=13.8, \mathrm{p}<.01$. This reflects the fact that HR was higher during the spider and food BAT as compared to the wood BAT. In addition, a linear trend emerged, $\underline{E}(1,36)=11.1, p<.01$, indicating that at the pre-treatment assessments, HR was higher during the spider BAT than during the food BAT. This linear trend disappeared immedi- 
ately after treatment, $\underline{F}(1,36)<1$. There was still some evidence for the presence of a quadratic trend, although the univariate F-tests did not reach the conventional level of significance, $\underline{F}(1,36)=2.6, p=.11$. At one week followup there was neither a linear nor a quadratic trend left $\underline{F} s(1,35)<1$. In other words, during the follow-up session, there were no differences in HR between the food, wood, and spider BAT (see Figure 6.3).

The MANOVA revealed a multivariate effect of treatment $F(6,30)=3.64$, $\mathrm{p}<.01$. Univariate $\underline{\mathrm{F}}$ tests indicated that there was no difference between the mean trends from the pre- to post-treatment assessments, $\mathrm{Fs}(1,35)<1$. Thus, there was no general decrease of HR after treatment. In the meantime, both the linear and the quadratic trends decreased immediately after treatment, $\underline{F}(1,35)=13.4, \mathrm{p}<.01$, and $\underline{F}(1,35)=9.7, \mathrm{p}<.01$, respectively. A similar pattern of results was evident at one week follow-up, $\mathrm{F}(1,35)=12.5, \mathrm{p}<.01$, and $\underline{\mathrm{F}}(1,35)=12.7, \mathrm{p}<.01$, respectively.

\section{Heart Rate}
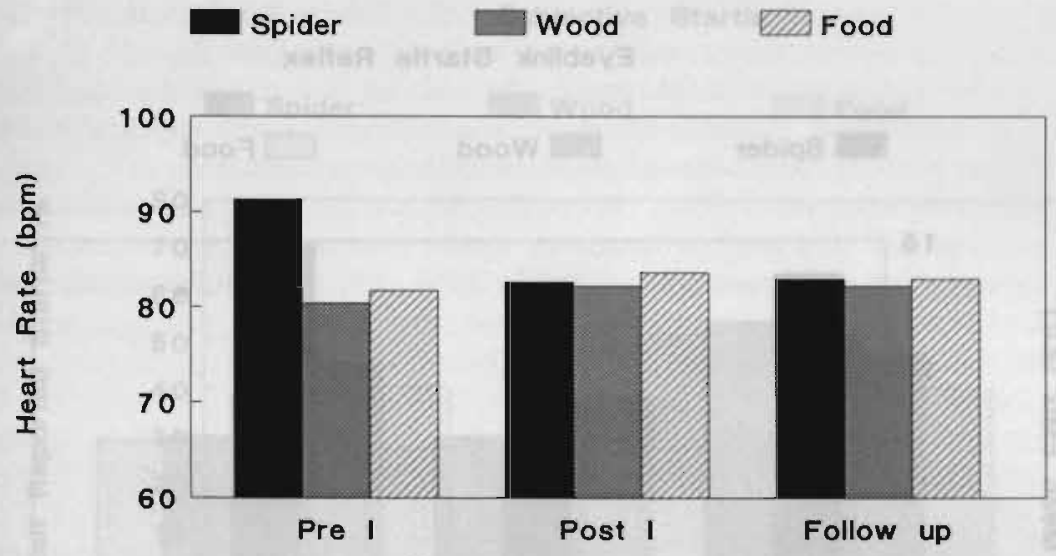

Figure 6.3. Heart rate during the spider, wood, and food BAT, before treatment, after treatment, and at one week follow up. Note that the distance to the spider was fixed for each subject and determined by subjects' pre-treatment achievement.

\section{Eyeblink startle responses}

Eyeblink startle responses are shown in Figure 6.4. During the pre-treatment assessment, a linear trend from spider to food was anticipated. Although the absence of a quadratic component $(\mathrm{F}(1,36)<1)$ indicated that the startle responses during the three different foreground stimuli did not significantly deviate from the hypothesized linear relationship between affective valence and startle magnitude, the linear trend did not attain significance, 
$\mathrm{F}(1,36)=1.06, \mathrm{p}=.30$.

During the assessment immediately after the treatment session, neither a linear nor a quadratic trend was found, $\underline{F} s(1,35)<1$. Thus, there were no differences in startle magnitude between the spider, wood, and food BATs.

At one week follow up, a linear trend was evident from spider to food, $\mathrm{F}(1,34)=4.1, \mathrm{p}<.05$. In other words, the startle magnitude during the spider BAT was larger than that during the food BAT. The absence of a quadratic trend $(\mathrm{F}(1,34)<1)$ indicated that there was no deviation from a linear relationship between startle magnitude and emotional valence.

Overall, there was a significant multivariate effect of treatment, $\mathrm{E}(6,28)=3.34, \mathrm{~g}<.05$. Univariate $\mathrm{F}$-tests indicated that this effect was carried by a difference in mean trends between the pre-treatment assessment and both post-treatment assessments, $\mathrm{E}(1,33)=14.9, \quad \mathrm{p}<.01$, and $\mathrm{F}(1,33)=12.1$, $\mathrm{p}<.01$, respectively. That is, in general, startle magnitudes were smaller immediately after treatment and at one week follow up than during the pretreatment assessment. Both the linear and the quadratic trends were not significantly affected by treatment, $\mathrm{Fs}(1,33)<1$.

\section{Eyeblink Startle Reflex}

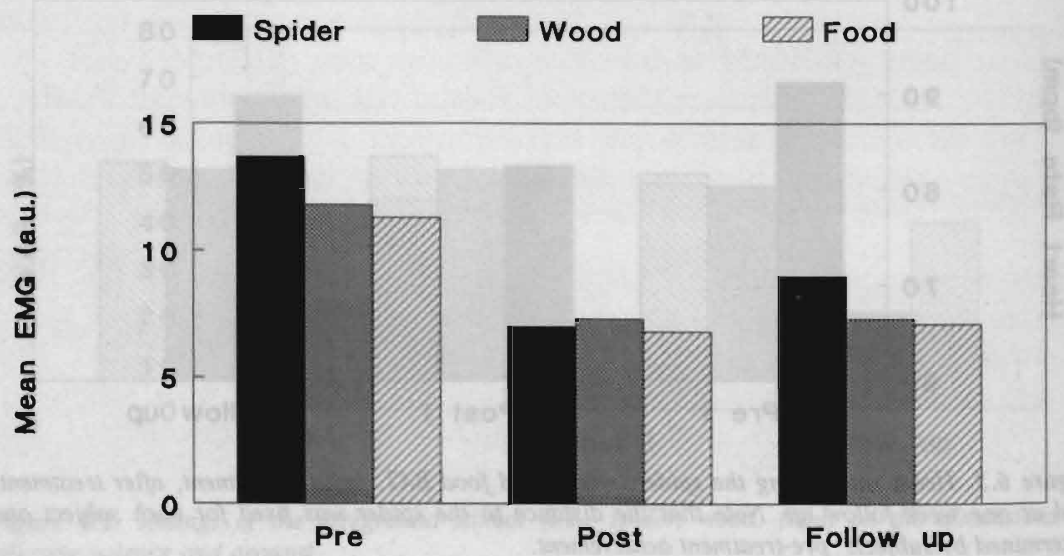

Figure 6.4. Mean magnitudes of the eyeblink startle responses during the spider, wood, and food $B A T$, before treatment, after treatment, and at one week follow up. Note that the distance to the spider was fixed for each subject and determined by subjects' pre-treatment achievement.

\section{Subjective startle reponse}

During the pre-treatment assessment a linear trend from spider to food BAT was evident $\underline{F}(1,36)=32.0, \mathrm{p}<.001$. In addition, a significant quadratic trend emerged, $\mathrm{F}(1,36)=5.1, \mathrm{p}=.03$, reflecting the finding that the difference 
between the spider and wood startles were larger than the difference between the food and wood startles (see Figure 6.5).

Immediately after the one-session treatment and at one week follow up, only linear trends reached significance, $\mathrm{F}(1,35)=7.8, \mathrm{p}<.01$, and $\mathrm{F}(1,34)=9.5, \mathrm{p}<.01$, respectively. This result reflects a linear relationship between self-reported startle and affective valence of the foreground stimuli.

Overall, there was a multivariate effect of treatment $F(6,28)=7.2$, $\mathrm{p}<.001$. Univariate $\mathrm{F}$-tests indicated that there was a significant difference in the mean trend from the pre- to the first post-treatment assessment, indicating a general decrease of the self-reported startle, $\underline{F}(1,33)=31.9, p<.001$. Yet, the mean trends of the pre-treatment and the second post-treatment assessments (at one week follow up) did not differ significantly, $E(1,33)=3.1$, $\mathrm{p}>.05$. For both post-treatment assessments, the slope of the linear trend was smaller than that of the pre-treatment assessment $\mathrm{F}(1,33), \mathrm{p}<.01$, and $\mathrm{F}(1,33)=8.6, \mathrm{p}<.01$, respectively. In other words, the differential responding between the spider and the food BAT decreased after treatment.

\section{Subjective Startle}

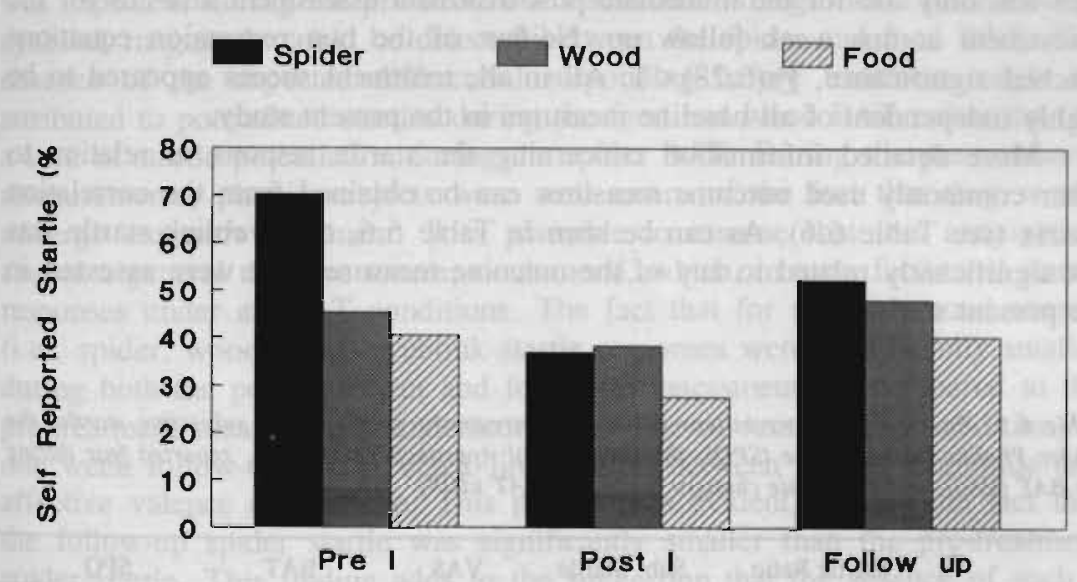

Figure 6.5. Self-reported startle responses during the pre- and post-trearment assessments and at one week follow up. Note that the distance to the spider was fixed for each subject and determined by subjects' pre-treatment achievement.

\section{Pre-treatment startle response and treatment effects}

Four (backward) regression analyses were carried out to investigate the predictive power of the startle response for immediate and short-term treatment succes. The first regression analysis was performed with the BAT difference score $\left(\mathrm{BAT}_{\text {pre }}\right.$ minus $\mathrm{BAT}_{\text {pros }}$ ) being the dependent variable and the 
pre-treatment startle response ratio, subjective startle, VAS, HR, SPQ, and pre-treatment BAT being the predictor variables. The equation that included all variables did not reach significance, $\mathrm{E}(6,28)<1$ and none of the predictor variables remained in the final equation. The second regression analysis tested the association of the behavioural improvement at one week follow up (BAT minus $\mathrm{BAT}_{\text {f.u. }}$ ) and the pre-treatment startle ratios, VAS, HR, SPQ, and pretreatment BAT. Only $20 \%$ of the short-term behavioural improvement could be explained by all independent variables operating jointly $(\underline{F}(6,28)=1.2$, $\mathrm{p}=.34$ ). In the backward analyses, none of the above mentioned predictor variables remained in the final equation. Consequently, it can be concluded that the startle ratios, VAS, HR, SPQ, and BAT can not satisfactorily predict behavioural improvement neither immediately after treatment nor at one week follow-up.

To explore the relationship between the improvement on the self-reported fear of spiders (i.e., SPQ) on the one hand and the pre-treatment startle ratios, BAT, VAS, HR, and SPQ on the other hand, two other (backward) multiple regression analyses were carried out. Dependent variables were the SPQs immediately after treatment $\left(S P Q_{p o s s}\right)$ and at one week follow up $\left(S P Q_{f, u}\right)$. The proportion of variance $\left(R^{2}\right)$ in SPQ scores explained by the dependent variables was only .08 for the immediate post-treatment assessment and .15 for the assessment at one week follow up. Neither of the two regression equations reached significance, $\mathrm{Fs}(6,28)<1$. All in all, treatment succes appeared to be highly independent of all baseline measures in the present study.

More detailed information concerning the startle respons in relation to other commonly used outcome measures can be obtained from the correlation matrix (see Table 6.6). As can be seen in Table 6.6, the eyeblink startle was not significantly related to any of the outcome measures that were assessed in the present study.

Table 6.6. Pearson p-m correlations between pre-treatment startle ratio, subjective startle, the Spider Phobia Questionnaire (SPQ), the Behavioural Approach Task (BAT), reported fear during the BAT (VAS), and heart rate changes during the BAT (dHR).

\begin{tabular}{llllll}
\hline & Startle Ratio & Sub. Stanle & VAS & BAT & SPQ \\
\hline Sub. Startle & 0.33 & & & & \\
VAS & 0.25 & .08 & & & \\
BAT & -0.27 & -.22 & $-0.48^{* *}$ & & \\
SPQ & .08 & .02 & $0.45^{* *}$ & $-0.67^{* *}$ & \\
dHR & .01 & -.06 & $0.49^{* *}$ & $-0.41^{*}$ & 0.28 \\
\hline
\end{tabular}

$*_{0}<<0.005$

$*^{*}<0.05$ 


\section{Discussion}

The major aim of the present study was to explore whether the startle probe methodology provides a useful psychophysiological index of therapy outcome and therapy prognosis in pathological anxiety. A prerequisite for such an index would be that it can be modulated by fear or anxiety. Pertinent to this issue, a study of Cook, Hawk, Davis, and Stevenson (1991) demonstrated that startle responses were larger during fear imagery than during pleasant imagery. Moreover, this effect appeared to be enhanced among subjects scoring high on the Fear Survey Schedule (FSS; Arrindell, Emmelkamp, \& van der Ende, 1984). A second criterion for a psychophysiological index of therapy outcome would be that the response is affected by successful treatment. Results from previous animal research strongly suggest that the startle probe response is affected by fear reduction. More specifically, Berg and Davis (1984) showed that fear induced startle potentiation can be blocked by anxiolytic drugs (i.e., diazepam).

In accordance with the animal research, the present study showed that the magnitude of the spider startles, indeed, decreased from the pre-treatment to both the post-treatment and follow-up assessments. Yet, in contrast to what was expected, during the pre-treatment assessment no significant difference in eyeblink magnitude could be detected between the spider and food BAT. The absence of the expected differential responding before treatment might be attributed to potentiated startles during the food and wood BAT rather than to a lack of startle potentiation during the spider BAT. During the pre-treatment measurements, phobic subjects were well aware of the fact that they would undergo exposure treatment. It is plausible to assume that this anticipation caused a general negative tone/anxious mood, which, in turn, facilitated startle responses under all BAT conditions. The fact that for all foreground stimuli (i.e., spider, wood, food) eyeblink startle responses were significantly smaller during both the post-treatment and follow-up assessments as compared to the pre-treatment measurement underscores this line of reasoning. In addition, at one week follow-up the expected linear trend between startle magnitude and affective valence did emerge. This pattern was evident, despite the fact that the follow-up spider startle was significantly smaller than the pre-treatment spider startle. This finding adds to the suggestion that the absence of such a linear trend during the pre-treatment assessment was due to a general fearinduced startle potentiation rather than to a failure of the spider BAT to potentiate startle probe responses.

Another explanation for the absence of differential responding before treatment might be, that the very high prior level of emotional excitation during the pre-treatment spider BAT, to some extent prevented foreground induced potentiation of the startle response to occur. Pertinent to this suggestion are Zillman's (1983) studies on "excitation transfer" in which it was shown that physiological responses to emotional stimuli are larger when they are evoked in moderate states of emotional excitation than in intense states of excitation (see Lang et al. 1990 , p. 389 , for a similar line of reasoning). This 
potential relationship between affect intensity and startle modulation, might also explain why in an earlier pilot study differential responding did occur during the pre-treatment assessment (de Jong et al. 1991). In that study, subjects were instructed to pull the spider as nearby as they normally would tolerate. In contrast, subjects in the present study were instructed that they should reach their limits. The latter instruction not only resulted in a "better" performance on the BAT (4.0 vs. 3.2) but also in higher levels of selfreported fear (VAS) during the BAT (62 vs. 51). It might well be that the higher level of fear due to the latter instruction (to some extent) prevented foreground induced startle potentiation to occur during the spider BAT.

In the present study, the startle reflex was not only indexed by the eyeblink response, but also by subjects' self-reported startle. The subjective startles appeared to be sensitive to the same factors as the eyeblink startle responses. More specifically, the self-reported startle responses varied systematically with the valence of the foreground stimuli. In fact, the relationship between subjective startle and affective valence was even more pronounced than that between the eyeblink startle and valence. Whereas at the pre-treatment assessment only a very weak linear relationship emerged between BAT valence and eyeblink startles, a very strong linear relationship was found between $\bar{B} \dot{A} \bar{T}$ valence and self-reported startles. Apparently, affectinduced potentiation of self-reported startles is less affected by the level of emotional excitation than is the affect-induced potentiation of eyeblink startles. Furthermore, the subjective startle appeared to be more specifically affected by treatment than the eyeblink component of the startle reflex. That is, only the self-reported spider startle (but not the wood or food startle) significantly decreased as a result of treatment.

The finding that the self-reported startle closely follows affective valence may be of practical relevance for the startle probe methodology. Obviously, on the one hand, self reported startle lacks some of the advantages of the eyeblink startle because it demands reflection, is vulnerable to experimental demand, and needs some explanation. On the other hand, subjective startles are easy to monitor, i.e., do not require technical facilities. In addition, it provides the opportunity to apply the startle probe methodology outside the laboratory (e.g., in a clinical setting).

To control for the possibility that the startle response (in the present BAT context) reflects arousal rather than affective valence, heart rate was measured as an index of autonomic arousal. In addition, subjects were asked to rate the foreground stimuli on the dimensions of affective valence and arousal. The pattern of the heart rate data were found to closely mimick the self-reported arousal. Subjects showed relatively low arousal during the wood BAT and relatively high arousal during the food and wood BAT, as indexed by both heart rate and self reported arousal. Meanwhile, the eyeblink startle responses closely followed the self-reported affective valence rather than the arousal evoking properties of the three different foreground stimuli. In other words, the present results indicate that the startle response is especially sensitive to affective valence and relatively independent of arousal, whereas the reverse is 
true for heart rate responses. Thus, the present data can be considered as further evidence for the position of Hamm (1992) that potentiation of the startle response indicates an avoidance (e.g., flight/fight) disposition induced by the evaluation of affective valence, while autonomic responding reflects general activation processes. In other words, the present findings are consistent with the view that the startle probe response is an "arousal free" index of emotional valence (e.g., Lang et al., 1990).

Some remarks are in order as to the clinical significance of the current data. The present study shows that the modulation of the startle probe response according to subjects' perception of positive and negative foreground stimuli is not restricted to the context of slide presentations and imagery. In addition, the startle reponse appeared to be relatively independent of other outcome measures (i.e., SPQ, BAT, HR). Furthermore, the present data suggest that the magnitude of the startle response is affected by treatment. Taken together, these findings suggest that the startle response might be a fruitful additional outcome variable, indexing aspects of phobia not covered by the more commonly used outcome measures. Yet, the present study could not replicate the earlier finding that pre-treatment startles have prognostic power for therapy outcome (de Jong et al., 1991). Neither treatment outcome indexed by post-treatment SPQ, nor indexed by post-treatment BAT scores were related to the pre-treatment startle response ratio. However, as said before, this absence of a relationship between the startle ratio and treatment outcome might be related to the relatively high level of prior emotional excitation during the pre-treatment spider BAT. In other words, it might well be that optimal startle potentiation (resulting in a more sensitive differentiation between subjects) only occurs at more moderate affective intensities. In order to draw more firm conclusions in this respect, parametric studies are needed concerning the relationship between affect intensity and startle modulation.

To recapitulate, the present findings confirm that in the context of a behavioural approach task the startle response predominantly reflects affective valence rather than arousal. In addition, the pattern of self-reported startles closely mimick the pattern of eyeblink startle responses. Furthermore, the present study suggests that fear-induced startle potentiation can be reduced by behavioural treatment. Finally, although the present results suggest that the startle response can be considered as a useful contribution to commonly used outcome measures, its prognostic properties remain to be established. 


\section{Epilogue: Two Years Follow Up}

To explore further the predictive properties of the startle probe reflex, subjects that participated in the study described above, were asked to complete the SPQ again at two years follow up. Although no relationship could be detected between the pre-treatment startle and the treatment effect at one week follow up, it can not be excluded that a relation between pre-treatment startle reflex and long term treatment effect does exist. For instance, it is conceivable that the SPQ completed one week after treatment is less reliable than $S P Q_{\text {follow }}$ up as subjects did not got much of a chance to test their new attitude against spiders in real live situations within only one week. Therefore, it seems reasonable to argue that at 18 months follow up the SPQ is reflecting more closely subjects real handling with spiders, whereas the $S P Q_{\text {post-rreatment }}$ is reflecting more subjects (perhaps incorrect) expectations (see de Jong et al, 1992 for a similar line of reasoning). Additionally, it might be that the startle magnitude is related to subjects' actual relapse after treatment.

\section{Method}

\section{Subjects}

From the 37 subjects that participated in the present "Spider Project" 32 subjects $(86 \%)$ responded to the request to complete a SPQ at two years follow up; the 5 remaining subjects could not be traced due to their moving to an unknown address. The treatment effect as indexed by the $S P Q_{\text {post-treatment }}$ and BAT $_{\text {post-reatment }}$ was similar for responders and "non-responders", means being 10.4 vs. 10.8 and 8.4 vs. 11.1 , ts $<1$.

\section{Procedure}

Immediately after the booster session at one week follow up, again, subjects underwent the BAT and completed the SPQ. All procedural details were similar to that of the above mentioned study. After 24 months all subjects were send a SPQ. Subjects who did not respond within one month were called by phone. This procedure was repeated until all subjects (with a known address) returned the SPQ.

\section{Analysis}

A backward regression analysis was carried out to investigate the prognostic properties of the pre-treatment startle response, subjective startle, SPQ, BAT, subjective reported fear during the BAT (VAS), and HR for treatment 
outcome as indexed by the SPQ at 24 months follow up. Because there are large differences in EMG responding among individuals, ratios of startle magnitude (i.e., mean magnitude spiderBAT/foodBAT) were preferred in the regression analyses. A second backward regression analysis was performed to explore whether relapse (as indexed by $S P Q_{\text {follow up }}$ minus $S P Q_{\text {post-reatment }}$ ) was related to post-treatment startle response, subjective startle, SPQ, BAT, and subjective reported fear during the BAT (VAS) (Post-treatment HR data were not available).

Finally, Pearson's p-m correlations were computed to investigate the interrelationship between the post-treatment subjective and eyeblink startle ratio and other more commonly used outcome measures; namely, the behavioural approach task (BAT), SPQ, and self reported fear during the BAT (VAS).

\section{Results and Discussion}

\section{Treatment Effects}

In general the treatment revealed a good immediate result. Mean SPQ decreased from 22.1 during the pre-treatment assessment to 10.8 after the booster session, $\mathrm{t}(31)=11.5, \mathrm{~g}<.001$. This effect was largely sustained at two years follow up, that is, no significant relapse occurred, $\mathrm{t}(31)<1$, means being 10.8 and 11.2 .

\section{Pre-treatment startle response and long-term treatment effect}

To explore the relationship between the improvement on the self-reported fear of spiders (i.e., SPQ) on the one hand and the pre-treatment startle ratios, BAT, VAS, HR, and SPQ on the other hand, a (backward) multiple regression analysis was carried out. Dependent variable was the SPQ at two years follow up. The proportion of variance $\left(R^{2}\right)$ in SPQ scores explained by the dependent variables was $.41, \underline{F}(6,24)=2.8, \mathrm{p}<.05$. Yet, only $S P Q_{\text {pre-treatment }}$ remained in the final equation (beta $=.57, \mathrm{p}<.001$ ). Thus, neither for the short-term nor the for long-term treatment effect, the startle ratios appear to have predictive properties.

The second regression analysis indicated that all post-treatment outcome measures acting together only explained $9 \%$ of the variance in relapse scores, $\underline{F}(5,25)<1$. None of the variables remained in the final equation.

More detailed information concerning the startle response in relation to other commonly used outcome measures and the $S P Q_{\text {follow up }}$ can be obtained from the correlation matrix (see Table 6.7). As can be seen in Table 6.7, the eyeblink startle was not significantly related to any of the outcome measures that were assessed in the present study nor to SPQ at two years follow up. 
Table 6.7. Pearson $p-m$ correlations between post-treatment startle ratio, subjective startle, the Spider Phobia Questionnaire (SPQ), the Behavioural Approach Task (BAT), reported fear during the BAT (VAS), and self reported fear of spiders at two years follow up (SPQfollow up).

\begin{tabular}{lllll}
\hline & $\begin{array}{l}\text { SPQpost-treat- } \\
\text { ment }\end{array}$ & $\begin{array}{l}\text { BATpost-treat- } \\
\text { ment }\end{array}$ & $\begin{array}{l}\text { VASpost-tre- } \\
\text { atment }\end{array}$ & SPQfollow up \\
\hline Startle Ratio & .15 & .02 & .19 & .05 \\
Sub. Startle & -.11 & .09 & .00 & -.09 \\
\hline
\end{tabular}

Taken together, the long-term follow up data do not provide any additional evidence to suggest that the startle response has prognostic properties for (long-term) treatment effects. 
7. CONTINGENCIES, COGNITIONS, \& REFLEXES: A RECAPITULATION

Parts of this chapter are published as: De Jong, P.J., \& Merckelbach, H. (1993). Covariation bias, classical conditioning, and phobic fear. Integrative Physiological and Behavioral Science, 28, $167-170$. 


\section{General Discussion}

The present thesis mainly focusses on two striking features of phobic fear that are difficult to reconcile with traditional learning accounts. The first issue concerns the persistence of fear in the absence of contingent aversive events. That is, why does phobic fear not extinguish in the presence of overwhelming evidence showing that the feared object is innoculous? The second issue pertains to the finding that on the one hand a large part of subjects suffering from phobias can not recall any conditioning experience (e.g., Merckelbach, de Ruiter, van den Hout, \& Hoekstra, 1989) whereas, on the other hand a substantial number of non-phobic subjects do recall such "conditioning events" but do not develop a phobia (e.g., Merckelbach, Arrindell, Arntz, \& de Jong, 1992). In addition to experiments that aim to shed some light on these issues, the present thesis contains two studies concerning a new index of phobic fear: The startle probe response. These two studies explored the evaluative and prognostic properties of the startle probe response in the treatment of spider phobia. In this final chapter the research findings that were presented in the previous chapters will be recapitulated and integrated; Potential clinical implications and suggestions for future research will be discussed. Note that the emphasis in this chapter will be on the first issue (i.e., the persistence of fear), as both other topics are already extensively discussed in Chapter 5 and 6.

\section{Covariation Bias, Classical Conditioning, and Phobic Fear}

Pavlovian conditioning is still one of the most influential theories on neurotic anxiety (e.g., Eysenck, 1987). In recent cognitive theories on conditioning, the phobic stimulus is considered as a predictor of aversive events (e.g., Eelen, 1982), or, in other words, as a danger signal (e.g., Reiss, 1980). Yet, it is evident that there is no sequential relationship between phobic stimuli and aversive events in real live. In fact, one of the DSM-III-R criteria for simple phobia is that the phobic fear is irrational. In order to reconcile this apparent inconsistency, it can be hypothesized that even in the absence of a systematic correlation, phobic subjects perceive phobic stimuli as predictors of aversive events. One major implication of such cognitive interpretation of classical conditioning is that subjects may associate a conditioned stimulus (CS) with an aversive unconditioned stimulus (UCS), without objective contingencies giving rise to this association.

\section{Experimentally induced illusory correlation}

To explore this assumption, we perf inducing an "illusory correlation" (IC) by presenting a "suggestive" series of CS/UCS pairings. Alloy and Tabachnik (1984) convincingly argued that the 
assessment of covariation is a function of prior expectations and current situational information (see also Alloy, 1988). As previous experience is an important factor in forming expectancies, their interactional model implies that "earliest covariation experiences have a disproportionately large impact on later contingency detection" (p.116). In line with this, animal studies have shown that truly random control procedures result in excitatory conditioning when CS-UCS pairings occur before non-pairings (Benedict \& Ayres, 1972). Similar primacy effects emerged in social psychological experiments; For instance, Anderson and Barrios (1961) found that personality impressions are more strongly affected by trait descriptors shown early rather than late in a presentation sequence. In a similar vein, Langer and Roth (1976) showed that the illusion of control is enhanced by early success in a purely chance task (i.e., coin tossing). Adopting an analoguous strategy the study described in Chapter 3.a attempted to induce an IC between a formerly neutral target stimulus and an aversive UCS: Subjects were exposed to two series of two different slides (CSs) randomly paired with the occurrence or non-occurrence of shock outcome (UCS). For both series, base rate probabilities of the two slides were equal, as were the conditional probabilities (i.e., $50 \%$ ). The first series (IC induction phase) started, however, with a number of pairings of one slide category (target slide) with shock. Across all trials, shock/slide contingency was equal for both slides (cf., Yates \& Curley, 1986). During the second phase, subjects were exposed to a random series of slide/shock pairings (IC extinction phase). Again, shock/slide contingency was equal for both slides.

The results of that study clearly indicated that it is possible to induce an "illusory correlation" between a formerly neutral stimulus and an aversive outcome. That is, subjects overestimated the covariation between the target stimulus and the aversive outcome, though the probability of shock outcome was equal for both stimuli. Most importantly, the results demonstrated that an IC once induced, can become "self-supporting": Subjects' on-line probability estimates of shock given the target slide increased during the second (extinction) phase, whereas the estimates of shock given the control slide declined. Thus, an acquired (illusory) UCS expectancy can act in such a way as to promote the assessment of differential associations. This finding sustains the hypothesis that subjects may associate a CS with an aversive UCS even in the complete absence of objective contingencies.

\section{Illusory correlation and phobia}

Following this line, it can be speculated that inflated UCS expectancies in phobics (e.g., as a result of conditioning experiences) likewise induce a bias to overestimate the contingency of phobia-relevant stimuli and aversive events. Such bias in information processing would be a particularly direct and powerful way to confirm or enhance fear. Recently, Tomarken and colleagues performed a series of experiments that sustains this suggestion (Tomarken, 
Mineka, \& Cook, 1989). In their studies, high and low snake-fearful college students were exposed to a large series of slides (CSs) of phobia-relevant and phobia-irrelevant stimuli. Immediately at slide offset one of three outcomes (UCSs) occurred: A tone, an aversive shock, or nothing at all. Although all stimulus/outcome combinations occurred equally often, subjects typically overestimated the covariation of fear-relevant stimuli and aversive outcomes, i.e., reported an illusory correlation. In line with the hypothesis that fearful subjects process information in a fear-confirming way, this bias was particularly strong in the high fear group. It seems reasonable to argue that this "illusory correlation" reflects a more general bias in high fear subjects to associate fear-relevant stimuli with aversive events.

To explore further the hypothesis that covariation bias might play a critical role in the maintenance of phobic fear, Chapter 2.a employed the same experimental procedure using clinical rather than analogue phobics. To the extent that phobic fear is responsible for the generation of the illusory correlation, one would expect the IC to be decreased as a result of successful treatment. Thus, half of the subjects were tested before and half of the subjects were tested after treatment. In addition, a no-fear control group of normal subjects was run. As expected, untreated subjects were, indeed, found to overestimate the spider-shock combinations. Surprisingly, treated subjects and no-fear controls displayed a similar bias to overassociate spiders and shock outcomes. Therefore, it appeared that phobic fear is not a crucial factor in the generation of a fear-relevant illusory correlation.

To avoid drawing the potentially premature conclusion that covariation bias is irrelevant to the maintenance of phobic fear, Chapter 2.b investigated two potential explanations for the absence of a treatment effect. First, although prior fear might be one source of covariation bias, alternative sources should be considered. For instance, cultural information might be an important additional source of preexperimental expectancy bias in the present context. Because of cultural connotations that spiders possess in the Netherlands, Dutch subjects may expect spiders to have aversive outcomes, irrespective of prior fear of spiders (cf., Davey, 1992). Following this line of reasoning, it may well be that cultural connotation and/or residual fear was sufficient to induce a considerable bias to associate spiders with shock in treated subjects and low-fear controls. In other words, it might be that the design employed in Chapter 2.a lacked sensitivity to detect treatment effects. In an attempt to enhance the sensitivity of the paradigm, the study presented in Chapter 2.b included additional danger-related CSs (i.e., aimed weapons). It seems reasonable to argue that, as a result of prior fear before treatment, spiders are more closely associated with aversive events than are both weapons and flowers. In the absence of prior fear, spiders and weapons may expected to be approximately equally related to danger, because of cultural connotations. Consequently, the presence of two competing threat-related stimuli is likely to undermine the covariation bias between spiders and shock after treatment (cf., Spears, van der Pligt, \& Eiser, 1985).

From Alloy and Tabachnik's framework a second explanation can be 
derived for the apparent absence of treatment effects in Chapter 2.a. That is, not only the quantitative overestimation of the spider-shock contingency but also subjects' confidence in the reported covariations may be important to the covariation bias phenomenon. It could be hypothesized that although treated subjects and low fear controls overestimated the spider/shock contingency to the same extent as untreated phobics, they were less confident about their estimates than were untreated subjects. Therefore, in Chapter 2.b, subjects were not only asked to report contingency estimates but also their confidence in these estimates.

In line with the predictions, Chapter 2.b showed that after the inclusion of a concurrent danger-related stimulus a treatment effect emerged. That is, in contrast to untreated subjects, treated subjects no longer typically overassociated spiders with shock. In fact, the data seem to imply, that as a result of treatment, the bias of associating spiders and shock relatively diminishes, whereas the estimates concerning the contingency between weapons and shock increase relatively. In addition, it was found that treated subjects were less confident concerning the covariation between stimuli and outcomes than untreated subjects. Subsequent research conducted along the same lines revealed a similar pattern of results (Chapters 3.d and 3.c). The finding that untreated but not treated phobics nor low-fear controls (Chapter 3.d) show a strong bias to associate phobia-relevant stimuli with shock is in line with the suggestion that phobic subjects process information in a fear confirming way. In addition, the high confidence of (untreated) phobics in detected covariations may add to the robust covariation bias in the maintenance of phobic fear. Meanwhile, in neither of these studies a relationship was found between the strength of the covariation bias and short-term therapy outcome. Thus, although a strong bias of associating phobic stimuli with aversive events may act in a way to confirm fear, it does not seem to be an obstacle for successful treatment.

Further research is needed to investigate whether covariation bias is restricted to animal phobias or can also be demonstrated in other anxiety disorders. From a clinical perspective, it would be of particular interest to study those phobic subjects who can not easily avoid the situations they strongly fear (e.g., blushing phobics), as it seems obvious that the impact of covariation bias is related to the frequency subjects encounter phobia-relevant situations.

\section{Pathways to covariation bias}

The bias to overassociate fear-relevant stimuli and aversive outcomes appears to be a robust phenomenon. An important issue that remains to be solved concerns the factors that underly this bias. Several mechanisms are captured by experimental studies. First, the overassociation between phobic cues and aversive events may gradually emerge as a result of random CS/UCS cooccurrences. In a number of studies it has been documented that perceivers 
overestimate the correlation between events that are both salient ("shared distinctiveness effect"; Hamilton \& Gifford, 1976) and/or have strong semantic associations (Chapman \& Chapman, 1967; Shweder, 1977; McArthur \& Friedman, 1980). Thus, the saliency of both spider slides and aversive shocks together with the "belongingness" of spiders and tactile outcomes (Hamm, Vaitl, \& Lang, 1989; Davey, 1994) might well result in subjects giving more weight to instances of spider slides followed by shock than to instances of fear-relevant cues followed by neutral outcomes or nothing at all. Such biased encoding of serially presented stimuli is likely to produce a posteriori reported illusory correlations (see e.g., Hamilton, Dugan, \& Trolier, 1985).

Second, subjects may have the strong expectation that phobic stimuli will be followed by aversive events and fail to correct this expectation (cf., McNally \& Heatherton, 1993; Spears, Eiser, \& van der Pligt, 1987). Third, at the time of judgment, biased retrieval may result in overestimating the relative frequency of CS/UCS co-occurrences. In a recent study, Schwartz, Bless, Strack, Klumpp, Rittenauer-Schatka, and Simons (1991) showed that the perceived ease of recall of pertinent information has a decisive influence on judgmental processes. On basis of this finding Schwartz et al. concluded "..what renders this heuristic prone to errors is that the experienced ease of retrieval may reflect the impact of variables other than frequency, such as the events' salience or vividness.." (p. 201). From this it might be inferred that the shared distinctiveness of spider slides and aversive shock (because of both salience and "belongingness") facilitates their recall which in turn may induce an illusory correlation between spiders and shock. A fourth factor that might underlie the development of illusory correlations between spider slides and shocks is the fact that both phobic cues and aversive UCSs induce a response pattern that can be characterized as a defense reflex (e.g., Graham, 1979). This match of the primary affective response organization (i.e., withdrawal) might result in a relatively strong (physiological) impact of shock outcome after spider slides (e.g., Lang, 1985). The larger impact presumably increases the saliency of the spider/shock pairings. This in turn, might promote the generation of an illusory correlation.

In sum, then, at least four mechanisms may be responsible for generating the a posteriori reported illusory correlation between fear-relevant stimuli and aversive outcomes: Expectancy bias, encoding bias, retrieval bias, and response similarities. Of course, the proposed mechanisms are not mutually exclusive and may well act in concert (e.g., Fiedler, Hemmeter, \& Hofmann, 1984). Furthermore, there is evidence to suggest that high levels of prior fear might intensify both expectancy bias (McNally \& Heatherton, 1993; Diamond, Matchett, \& Davey, 1993) and encoding bias (cf., Mackie, Hamilton, Schroth, Carlisle, Gersho, Meneses, Nedler, \& Reichel, 1989).

Preexperimental and on-line expectancies. Chapter 3.b explored the role of prior expectations in the postexperimentally reported illusory correlations (e.g., Chapter 2.b) by means of a "thought experiment" adopted from McNally and Heatherton (1993). In the Chapter 3.b study, high and low spider- 
fearful subjects were asked to read a description of the experimental procedure of a previous IC-experiment (cf. Chapter 2.b) and were asked to predict the likely probabilities of all slide/outcome combinations. The pattern of the reported slide/outcome expectations in Chapter 3.b turned out to be highly similar to that of the postexperimentally reported covariation estimates of the high-fear subjects in the Chapter 2.b study. This finding corroborates the conclusion previously drawn by McNally and Heatherton (1993) that "biased covariation estimates might be at least partly attributed to preexperimental expectancy biases, and not arise solely from biased on-line processing". Yet, the finding that the preexperimental expectancies were similar and equally strong for both high-fear and low-fear subjects suggests that postexperimentally reported illusory correlations not merely reflect preexperimental expectancies. Note that in the de Jong et al. (1992) study, only high-fear subjects showed an a posteriori bias to overassociate spiders with shock, while low-fear subjects were in fact quite accurate. Taking together, the results of Chapters 2.b and 3.b suggest that the biased preexperimental spider/shock expectancies of high-fear subjects are especially resistant to disconfirmation. Because high-fear and low-fear subjects appear to have an equally strong preexperimental expectancy bias, processes during the actual experimental procedure are apparently responsible for the between-groups differences regarding the postexperimentally reported covariation estimates.

Chapter 3.c investigated further to what extent differential preexperimental expectancies underlie the illusory correlation phenomenon. In addition, Chapter 3.c explored whether differential encoding during the experiment and response similarity to stimuli and outcomes contribute to the biased covariation detection. Therefore, the Chapter 3.c study was designed to seperately monitor initial expectations, on-line processing at the cognitive and physiological level, and post-experimental contingency judgment. The results of Chapter 3.c show that right from the beginning of the experiment the majority of phobic subjects expected a shock after the spider slide. However, the same held true for the weapon slide, whereas only a minority of phobics expected a shock after the first flower slide. Most pertinent for the present discussion, the phobic subjects seemed to correct their initial shock expectancies on weapon trials but not on phobia-relevant trials. That is, the post-experimentally reported weapon-shock contingency estimates were quite correct, whereas the spider-shock estimates were inflated. The on-line reported shock expectancies confirmed this pattern: While subjects' expectancy of shock after weapon trials rapidly declined to the frequency one would expect in subjects making a random choice $(1 / 3)$, they continued to expect shocks after approximately half of the spider slides. Thus, it appears that random slide/outcome presentations are sufficient to eliminate weapon-shock expectancy biases whereas random presentations are relatively ineffective to eliminate UCS expectancy biases on phobia-relevant trials. In other words, the present findings suggest that the a posteriori reported illusory correlation between spiders and shock arises from initial expectancies that survive extinction. 
ming covariation bias, is to provide immediate feedback on a very large number of predictions of which the confidence level is carefully recorded (cf., Wagenaar \& Keren, 1986). Homework assignments in combination with diary recordings is one clinical application of this technique. In such cases, careful recordings of predictions and outcomes are vital as the use of unaided memory for coding, storing, and retrieving outcomes is highly vulnerable for distortion in a way to confirm prior expectations (e.g, Einhorn, 1978; Golding \& Rorer, 1972).

On many occasions the causative status of cognitions in psychopathology is seriously questioned. One of the major criticisms pertains to the concepts used in the research on the role of cognitions in psychopathology; It is often difficult to distinguish between the symptomatology of certain syndromes and the cognitions that are proposed to have caused these syndromes. Costello (1992) argued that there is often a tautological relationship between symptoms and cognitions in the literature (cf., Fiedler, 1991). A study of Chambless and colleagues may serve to illustrate his concern. In a factor analysis, Chambless, Beck, Gracely, and Bibb (1991) found that semantically related descriptions of cognitions (e.g., I am going to have a heart attack) and bodily symptoms (e.g., heart palpitations) loaded on the same factors. They interpreted these data as supportive evidence for the view that a catastrophic misinterpretation of bodily symptoms is a cause of panic. However, note that it is not at all clear that the indices of bodily symptoms and cognitions in this study are measuring different concepts, therefore, Costello quite rightly objected that "...a more plausible interpretation of their findings must surely be that the self-report items such as I am going to have a heart attack and heart palpitations denote the same experience" (p. 385).

Although we share the concern that research on the causative role of cognitions will bring us no much further if the predicting and the to be predicted variables map on indistinguishable experiences, it appears that this drawback is typically pertinent to research that taps cognitions by means of self-report instruments. Even apart from the potential problem of indistinguishability, self-report measures of cognitions may also be compromised by their susceptibility to demand, distortion, and attributional biases (e.g., Arntz, Rauner, \& van den Hout, 1994). Furthermore, pertinent cognitive processes are not necessarily accessible for conscious awareness. Therefore, it seems most fruitful to rely on performance measures rather than introspection, to explore cognitive vulnerability factors in the etiology of psychopathology (cf., Eysenck, 1992). The IC-paradigm (e.g., Chapter 4) and the modified Stroop task (e.g., MacLeod \& Hagan, 1992) are two examples of paradigms that tap (biased) cognitive processing via performance measures. Paradigms from reasoning research should also be considered, as many of these reasoning tasks may be well adapted for investigating the relationship between biased reasoning and psychopathology. Following this strategy, de Jong, Mayer, and Bögels (1994), recently employed a Wason selection task to explore the relationship between maladaptive deductive reasoning patterns and phobic fear (see also Quelhas \& Power, 1992; Arntz, de Jong, Merckelbach, \& Rauner, 
1993). Given the lack of reliable, theory-derived predictors of anxiety development and relapse, and given the clinical relevance of such predictors, efforts to replicate and extend this kind of research would be very welcome.

\section{Cognitive bias, hemisphere preference, and phobic fear}

From a more theoretical level, it might also be worthwile to explore whether cognitive biases can be pinpointed to neuropsychological mechanisms. Recent studies found that subjects with a right hemisphere preference (indexed by Zenhausern's Preference Test) report more fear symptoms than subjects with a left hemisphere thinking style (Merckelbach et al., 1991; Chapter 3.d). Studies that more directly assessed hemisphere activation (e.g., baseline EEG) also sustain the hypothesis that especially the right hemisphere is involved in negative emotions. For example Tomarken, Davidson, and Henriques (1990; see also Davidson, 1988) showed that the threshold for expressing anxiety is lowered in subjects showing a relatively strong right hemisphere activation. In addition, a host of studies (e.g., this thesis) have demonstrated that anxiety disordered subjects tend to rely on dysfunctional cognitive rules. For example anxious subjects selectively process threatening information (e.g., attentional bias) and dramatically overestimate the association of phobic stimuli and aversive events (covariation bias). It may well be that a preference for a right hemisphere mode of thinking promotes biased cognitive processing, which in turn, lead to dysphoric emotions. It would be interesting to see if cognitive biases, indeed, are a function of the right hemisphere and to what extent the relationship between hemisphericity and phobic fear is mediated by such biases (see Chapter 3.d for a more detailed discussion).

\section{Final Remarks}

The Introduction presents an heuristic model of phobic fear that essentially distinguishes two stages: contingency evaluation and UCS evaluation. The present thesis revealed the following results:

\section{Contingency Evaluation}

(1) Incidental CS-UCS co-occurrences can result in illusory contingency judgments (Chapter 3.a).

(2) Once induced, such illusory correlations can become self-supporting (Chapter 3.a).

(3) A priori expectations promote the development of fear-confirming illusory correlations (Chapter 3.c). 
(4) In their turn, expectancy biases can result from conditioning-like experiences, prior fear, and cultural connotations (Chapters 3.a, 3.b, \& 3.c).

(5) Similarity of responses to CSs and UCSs may sustain biased contingency judgments (Chapters 3.c, 2.a, \& 2.b).

(6) Phobic subjects show an illusory correlation between phobic cues and aversive events. The IC seems susceptible to behavioural treatment (Chapter 2.b).

(7) In general, subjects show a possitive correlation between indices of phobic fear and the association of fear-relevant stimuli and aversive outcomes (Chapter 3.d, exp.2).

(8) There is a reciprocal relationship between covariation bias and phobic fear; Thus, the perception of illusory correlations is not merely a concommitant of phobic fear (Chapter 4.a).

Taken together, the results presented in this thesis provide an empirical basis for the suggestion that human contingency evaluation processes may lead to a "phobogenic" misrepresentation of CS-UCS contingencies. The present findings not only suggest how incidental CS-UCS co-occurrences may be of importance in the etiology of phobic fear, they also provide an explanation for the persistence of phobic fear in the absence of contingent aversive events.

\section{UCS Evaluation}

Whereas strong evidence was found for the significant role of biased contingency evaluation in phobias, evidence for UCS inflation scenarios in the etiology of phobic fear proved to be, at best, rather weak. The present thesis revealed the following results pertaining to the second stage (i.e., UCS evaluation) of the heuristic human conditioning model of phobic fear:

(1) It is often possible to reconstruct the anamnesis of anxiety disordered patients along the lines of an UCS inflation scenario (Chapter 5.a; see also Davey, de Jong \& Tallis, 1993).

(2) In accordance with the model, experiment 1 (Chapter 5.a) demonstrated that UCS inflation promotes resistance to extinction of the CR. 
(3) Subsequent research employing a similar paradigm, yet even with a larger sample size, could not replicate these positive results (Chapter 5.b).

(4) Further experiments designed to enhance the 'ecological validity' of the UCS inflation paradigm failed to provide convincing evidence to suggest that human conditioned responding is susceptible to UCS inflation (Chapter 5.a, experiments 2-4).

(5) Even if potentially relevant personality characteristics were taken into account, no reliable inflation effect could be detected (Chapter 5.b).

The finding that it is often possible to interpret the learning history of anxiety disordered patients in terms of an inflation scenario may seem to be convincing support for the potential importance of UCS inflation in the etiology of anxiety disorders. However, it should be stressed that such cases are gratuitous as long as there is no solid independent empirical evidence for inflation phenomena affecting fear conditioning in humans, and, thus far, the empirical basis of UCS-inflation in humans is quite meagre. In addition, even if one is ready to accept that UCS-inflation phenomena play a role in the etiology of anxiety disorders there is a more fundamental point that can be raised. That is, there is no clear definition of what should be considered as UCS inflation events. Therefore, one runs the risk of becoming a victim of the fallacy of petitio principii: A UCS inflation scenario is advanced to account for the etiology of an anxiety disorder, whereas this approach is legitimized by the mere presence of that anxiety disorder (see Chapter 5.a for a more detailed discussion). All in all, it can be concluded that there is no solid empirical basis to integrate UCS-inflation in human conditioning models of anxiety disorders. Therefore, unless proponents of a UCS inflation model come up with new evidence, it seems most worthwhile for future research to focus on factors influencing the evaluation of contingencies rather than isolated UCS experiences. 


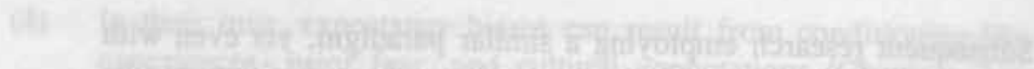

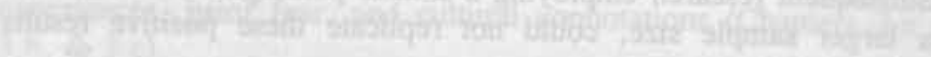

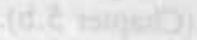

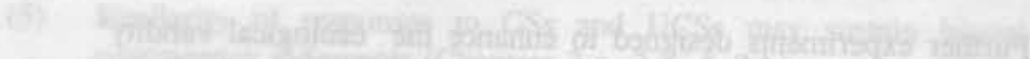

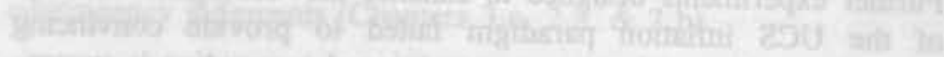

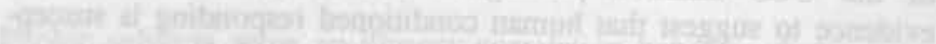

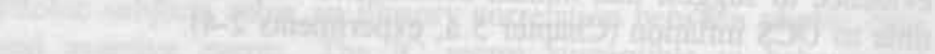

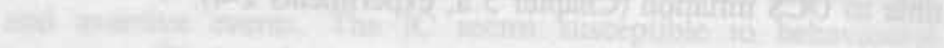

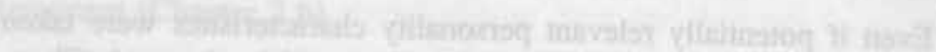

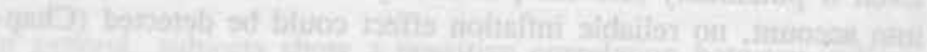

C.

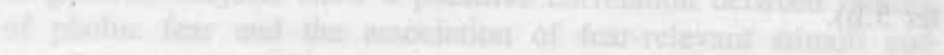

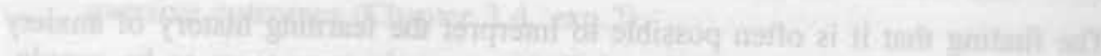

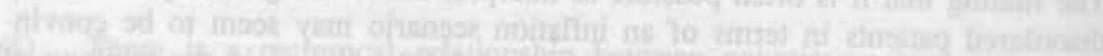

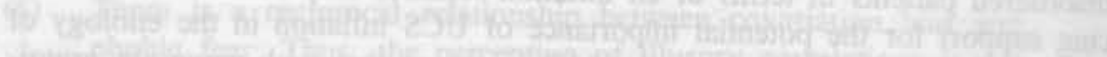

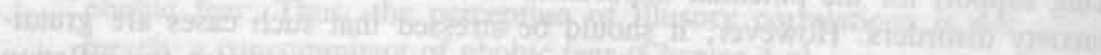

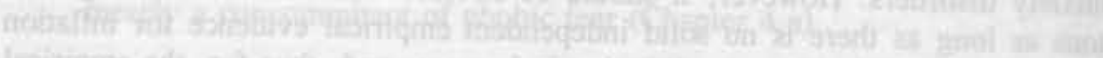

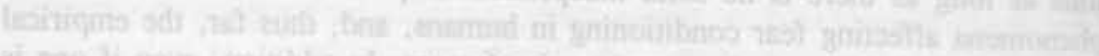

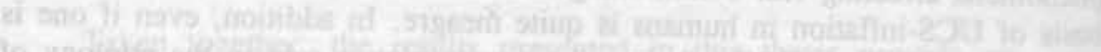

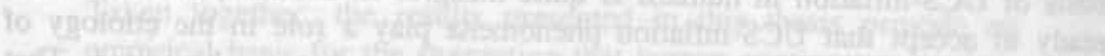

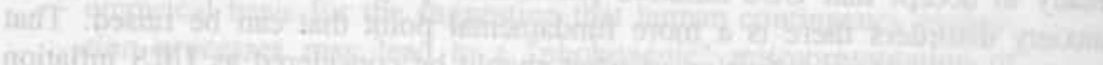

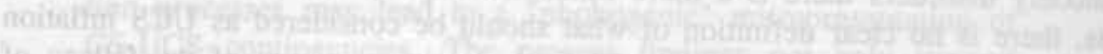

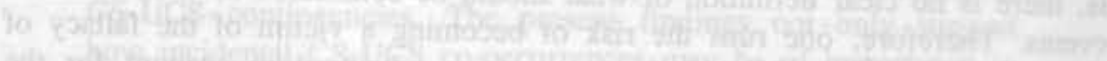

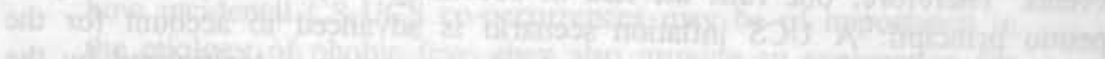

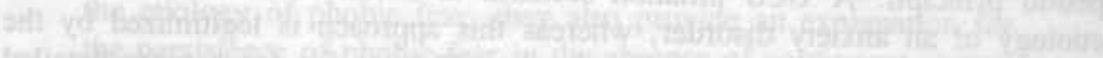

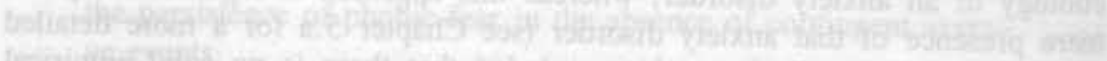

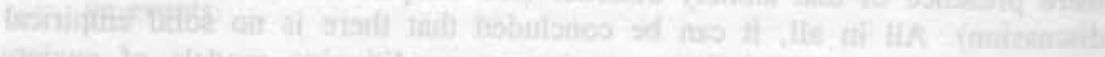

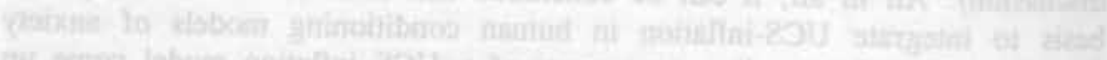

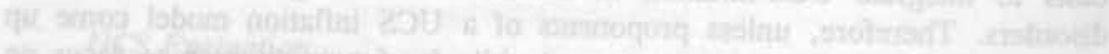

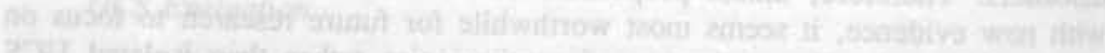

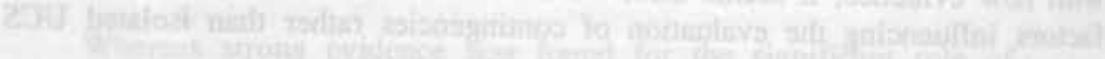
an

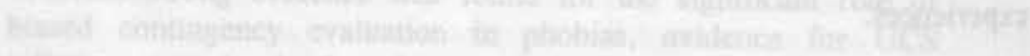

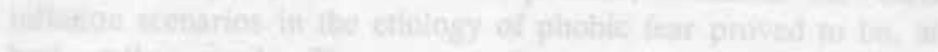

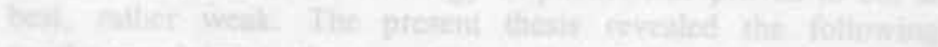

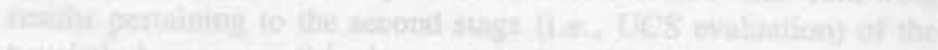

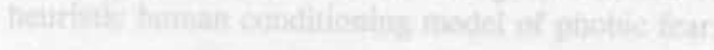

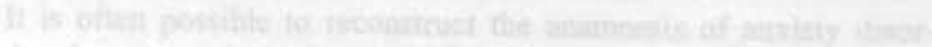

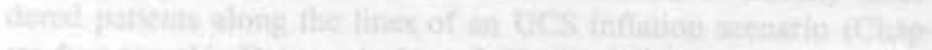

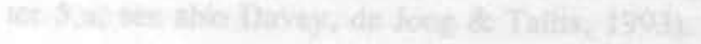

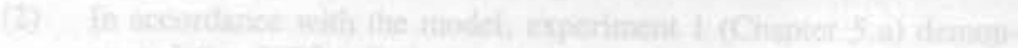

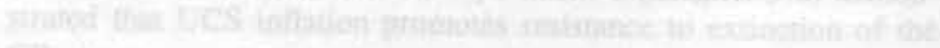
VIII 


\section{REFERENCES}

Allan, G.A. (1993). Human contingency judgment: Rule based or associative? Psychological Bulletin, 114, 345-448.

Algom, D., \& Bizman, A. (1983). Attribution theory: A conditioning interpretation. Perceptual and Motor Skills, 56, 767-774.

Alloy, L.B. (1988). Expectations and situational information as cocontributors to covariation assessment: A reply to Goddard and Allan. Psychological Review, 95, 299-301.

Alloy, L.B., \& Abramson, L.Y. (1979). Judgment of contingency in depressed and nondepressed students: Sadder but wiser? Journal of Experimental Psychology: General, 108, 441-485.

Alloy, L.B., Abramson, L.Y., \& Kosman, D.A. (1985). The judgment of predictability in depressed and nondepressed college students. In F.R Brush and J.B. Overmier (Eds.), Affect, conditioning, and cognition: Essays on the determinants of behavior. London: LEA.

Alloy, L.B., Abramson, L.Y., \& Viscusi, D. (1981). Induced mood and the illusion of control. Journal of Personality and Social Psychology, 41, 1129-1140.

Alloy, L.B. \& Tabachnik, N. (1984). Assessment of covariation by humans and animals: The joint influence of prior expectations and current situational information. Psychological Review, 91, 112-149.

American Psychiatric Association (1987). Diagnostic and statistical manual of mental disorders (Rev. 3rd ed.). Washongton, DC: Author.

Anderson, N.H., \& Barrios, A.A. (1961). Primacy effects in personality impression formation. Journal of Abnormal and Social Psychology, 63, 346-350.

Arkes, H.R. (1991). Costs and benefits of judgment errors: Implications for debiasing. Psychological Bulletin, 110, 486-498.

Arkes, H.R., \& Harkness, A.R. (1983). Estimates of contingency between two dichotomous variables. Journal of Experimental Psychology General, 112, 117-135.

Arntz, A., de Jong, P.J., Merckelbach, H., \& Rauner, M. (1993). Biological and cognitive factors in the development and maintenance of anxiety disorders. Paper presented at the 23rd European Congress of Behaviour and Cognitive Therapies, London (September).

Arntz, A., Rauner, M., \& van den Hout, M.A. (1994). 'If I feel anxious, there must be danger': The fallacy of ex-consequentia reasoning in inferring danger in anxiety disorders. Manuscript under review.

Arntz, A., van den Hout, M.A., van den Berg, G., \& Meijboom, A.C. (1991). The effects of incorrect pain expectations on acquired fear and pain responses. Behaviour Research and Therapy, 29, 547-560.

Arrindell, A., Emmelkamp, P.M.G., \& van der Ende, J. (1984). Phobic dimensions: I. Reliability and generalizability across samples, gender and nations. Advances in Behaviour Research and Therapy, 6, 207-253.

Baeyens, F., Eelen, P., van den Berg, O., \& Crombez, G. (1992). The content of learning in human evaluative conditioning: acquired valence is sensitive to US-revaluation. Learning \& Motivation, 23, 200-224.

Bakan, P. (1978). Two streams of consciousness. A typological approach. In K.S. Pope \& J.L. Singer (Eds.). The stream of consciousness. New York: Wiley 
Beaumont, J.G., Young, A.W., \& McManus, I.C. (1984). Hemisphericity: A critical review. Cognitive Neuropsychology, 1, 191-212.

Benedict, J.O., \& Ayres, J.J.B. (1972). Factors affecting conditioning in the truly random control procedure in the rat. Journal of Comparitive and Physiological Psychology, 78, 323-330.

Berg, W.K. \& Davis, M. (1984). Diazepam blocks fear-enhanced startle elicited electrically from the brainstem. Physiology and Behavior, 32, 333-336.

Bouton, J. (1984). Differential control by context in the inflation and reinstatement paradigms. Journal of Experimental Psychology, 10, 56-74.

Bouton, M.E., \& Swartzentruber, D. (1991). Sources of relapse after extinction in Pavlovian and instrumental learning. Clinical Psychology Review, 11, 123140.

Bradley, M.M., Cuthbert, B.N., \& Lang, P.J. (1990) Startle reflex modification: Emotion or attention? Psychophysiology, 27, 513-522.

Brewin, C. R. (1988). Cognitive foundations of clinical psychology. Hillsdale: Lawrence Erlbaum Associates Inc.

Campbell, D.E. \& Beets, J.L. (1978). Lunacy and the moon. Psychological Bulletin, 85, 1123-1129.

Chambless, D.L., Beck, A.T., Gracely, E.J., \& Bibb, J.L. (1991). The relationship of cognitions to fear of somatic symptoms: A test of the cognitive theory of panic. Unpublished manuscript.

Chapman, L.J. (1967). Illusory correlation in observational report. Journal of Verbal Learning and Verbal Behavior, 6, 151-155.

Chapman, L.J., \& Chapman, J.P. (1967). Genesis of popular but erroneous psychodiagnostic observations. Journal of Abnormal Psychology, 72, 193-204.

Chapman, L.J., \& Chapman, J.P. (1969). Illusory correlation as an obstacle to the use of valid diagnostic signs. Journal of Abnormal Psychology, 74, 271-280.

Clark, D.M. (1988). A cognitive model of panick attacks. In: S. Rachman \& J.D. Maser (Eds.), Panic: Psychological Perspectives. Hillsdale: Erlbaum.

Cook, E.W., Hawk, L.W., Davis, T.L., \& Stevenson, V.E. (1991). Affective individual differences and startle reflex modulation. Journal of Abnormal Psychology, 100, 5-13.

Cook, E.W., Hodes, R.L., \& Lang, P.J. (1986). Preparedness and phobia: Effects of stimulus content on human visceral conditioning. Journal of Abnormal Psychology, 95, 195-207.

Cook, E.W., Melamed, B.G., Cuthbert, B.N., McNeil, D.W., \& Lang, P.J. (1988). Emotional imagery and the differential diagnosis of anxiety. Journal of Consulting and Clinical Psychology, 56, 734-740.

Coren, S. \& Ward, L.M. (1989). Sensation and Perception (p.313). San Diego: Harcourt Brace Jovanovich.

Costello, C.G., (1992). Conceptual problems in current research on cognitive vulnerability to psychopathology. Cognitive Therapy and Research, 16, 379390.

Davey, G.C.L. (1987). An integration of human and animal models of Pavlovian conditioning: Associations, cognitions, and attributions. In G. Davey (Ed.), Cognitive processes and Pavlovian conditioning in humans. Chichester: Wiley.

Davey, G.C.L. (1988a). Integrating contemporary models of animal and human conditioning: Implications for behavior therapy. In P. Emmelkamp. W. 
Everaerd, F. Kraaimaat \& M. van Son (Eds.). Advances in Theory and Practice in Behavior Therapy. Amsterdam: Swets, p. 37-43.

Davey, G.C.L. (1988b). Pavlovian conditioning in humans: UCS revaluation and the self-observation of conditioned responding. Medical Science Research, $16,957-961$.

Davey, G.C.L. (1989). UCS revaluation and conditioning models of acquired fears. Behaviour Research and Therapy, 27, 521-528.

Davey, G.C.L. (1992a). An expectancy model of laboratory preparedness effects. Journal of Experimental Psychology: General, 121, 24-40.

Davey, G.C.L. (1992b). Classical conditioning and the acquisition of human fears and phobias: A review and synthesis of the literature. Advances of Behaviour Research and Therapy, 14, 29-66.

Davey, G.C.L. (1993). Cognitive judgmental predictors of 'belongingness' in phylogenetic and ontogenetic stimuli. Manuscript under review.

Davey, G.C.L., de Jong, P.J., \& Tallis, F. (1993). UCS inflation in the aetiology of a variety of anxiety disorders: Some case histories. Behaviour Research and Therapy, 31, 495-498.

Davey, G.C.L. \& McKenna, I. (1983). The effect of postconditioning revaluation of CSI and UCS following Pavlovian second-order electrodermal conditioning in humans. Quaterly Journal of Experimental Psychology, 35, 125-133.

Davidson, R.J. (1988). EEG measures of cerebral asymmetry: Conceptual and methodological issues. International Journal of Neuroscience, 39, 71-89.

Davidson, R.J. \& Fox, N.A. (1982). Asymmetrical brain activity discriminates between positive versus negative affective stimuli in human infants. Science, $218,1235-1237$.

Davidson, R.J. \& Fox, N.A. (1989). Frontal brain asymmetry predicts infants' response to maternal separation. Journal of Abnormal Psychology, 98, 127131.

Davis, M. (1984). The mammalian startle response. In R.C. Eaton (Ed.), Neural mechanisms of startle behavior (pp. 287-351). New York: Plenum Press.

Davis, M., Hitchcock, J., \& Rosen, J.B. (1989). Anxiety and the amygdala: Pharmacological and anatomical analysis of fear-potentiated startle. In G.H. Bower (Ed.), The Psychology of learning and motivation: Advances in research and theory (Vol. 21, pp. 263-305). San Diego, CA: Academic Press.

Dawson, M.E., Schell, A.M, \& Banis, H.T. (1986). Greater resistance to extinction of electrodermal responses conditioned to potentially phobic CSs: A noncognitive proces? Psychophysiology, 23, 552-561.

De Jong, P.J., (1993). Covariation bias in phobia: Mere resistance to pre-experimental expectancies? Behavior Therapy, 24, 447-454.

De Jong, P.J., Arntz, A., \& Merckelbach, H. (1991). The startle response: Its prognostic and evaluative properties in the treatment of phobia. Paper presented at the International Congress on Stress, Anxiety, and Emotiona' Disorders, Braga, Portugal.

De Jong, P.J., Arntz, A., \& Merckelbach, H. (1993). The startle prole response as an instrument for evaluating exposure effects. Advances in Behaviour Research and Therapy, 15, 301-316.

De Jong, P.J., Mayer, B., \& Bögels, S.M. (1994). Biased reasoning patterns and phobic fear. Paper presented at the 36 . Tagung der experimentell arbeitender 
Psychologen, München (April).

De Jong, P. \& Merckelbach, H. (1991). Covariation bias and electrodermal responding in spider phobics before and after behavioural treatment. Behaviour Research and Therapy, 29, 307-314.

De Jong, P.J. \& Merckelbach, H. (1991). Covariation detection and spider phobia. Paper presented at the International Congress on Stress, Anxiety, and Emotional Disorders, Braga, July.

De Jong, P.J. \& Merckelbach, H. (1993). Covariation bias, classical conditioning, and phobic fear. Integrative Physiological and Behavioral Science, 28, 167 170.

De Jong, P., Merckelbach, H., \& Arntz, A. (1990). Illusory correlation, on-line probability estimates, and electrodermal responding in a (quasi) conditioning paradigm. Biological Psychology, 31, 201-212.

De Jong, P.J., Merckelbach, H, \& Arntz, A. (1991). Eyeblink startle responses in spider phobics before and after treatment: A pilot study. Journal of Psychopathology and Behavioral Assessment, 13, 213-223.

De Jong, P.J., Merckelbach, H., Arntz, A., \& Nijman, H. (1992). Covariation detection in treated and untreated spider phobics. Journal of Abnormal Psychology, 101, 724-727.

De Jong, P., Merckelbach, H., \& Muris, P. (1990). Conjugate lateral eye movements, cerebral dominance, and anxiety. In R.J. Takens (Ed.). European Perspectives in Psychology; Vol 2, (pp. 38-48) New York: Wiley.

De Jong, P.J., van den Hout, M.A., \& Merckelbach, H. (1994). Covariation bias and the return of fear. Behaviour Research and Therapy, in press.

Diamond, D., Matchett, G., Davey, G.C.L. (1993). The effect of prior fear levels on UCS-expectancy ratings to a fear-relevant stimulus. Manuscript under review.

Dickinson, A.K. \& Shanks, D. (1985). Animal conditioning and human causality judgment. In L.G. Nilsson \& T. Archer (Eds.), Perspectives on learning and memory (pp. 167-191). London: Erlbaum.

Dimberg, U. (1986). Facial expressions as excitatory and inhibitory stimuli for conditioned autonomic responses. Biological Psychology, 22, 37-57.

Dimond, S.J. \& Farrington, L. (1977). Emotional responses to films shown to the right or left hemisphere of the brain measured by heart rate. Acto Psychologica, 41, 255-260.

Donnell, C.D. \& McNally, R.J. (1990). Anxiety sensitivity and panic attacks in a nonclinical population. Behaviour Research and Therapy, 28, 83-85.

Drake, R.A. (1987). Effects of gaze manipulation on aesthetic judgments: Hemisphere priming of affect. Acta Psychologica, 65, 91-99.

Edelmann, R.J., (1990). Chronic blushing, self-consciousness, and social anxiety. Journal of Psychopathology and Behavioral Assessment, 12, 119-127.

Eelen, P. (1982). Conditioning and attribution. In Boulangouris, J. (Ed.), Learning theory approaches to psychiatry (pp. 3-17). New York: Wiley.

Edwards, J.M., \& Endler, N.S. (1989). Appraisal of stressful situations. Personality and Individual Differences, $10,7-10$.

Ehrlichman, H. \& Barret, J. (1983). Right hemispheric specialization for mental imagery: A review of the evidence. Brain and Cognition, 2, 55-76.

Einhorn, H.J., Hogarth, R.M. (1978). Confidence in judgment: Persistence of the illusion of validity. Psychological Review, 85, 395-416. 
Eysenck, H.J. (1976). The learning theory model of neurosis: A new approach. Behaviour Research and Therapy, 14, 252-267.

Eysenck, H.J. (1987). Behavior Therapy. In H.J. Eysenck \& I. Martin (Eds.), Theoretical Foundations of Behavior Therapy (pp 3-35). New York: Plenum.

Eysenck, M.W. (1992). Anxiety: The cognitive perspective. Hillsdale: LEA.

Farah, M.J. (1984). The neurological basis of mental imagery: A componential analysis. Cognition, 18, 245-272.

Fiedler, K. (1991). Heuristics and biases in theory formation: On the cognitive processes of those concerned with cognitive processes. Theory \& Psychology, 1, 407-430.

Fiedler, K., Hemmeter, U., Hofmann, C. (1984). On the origen of illusory correlation. European Journal of Social Psychology, 14, 191-201.

Foa, E.B., \& Kozak, M.J. (1986). Emotional processing of fear: Exposure to corrective information. Psychological Bulletin, 99, 20-35.

Foa, E.B., \& McNally, R.J. (1986). Sensitivity to feared stimuli in obsessivecompulsives: A dichotic listening analysis. Cognitive Therapy and Research, $10,477-485$.

Fowles, D.C., Christie, M.J., Edelberg, R., Grings, W.W., Lykken, D.T., \& Venables, P.H. (1981). Publication recommendations for electrodermal measurements. Psychophysiology, 18, 232-239.

Fredrikson, M. (1983). Reliability and validity of some specific fear questionnaires. Scandinavian Journal of Psychology, 79, 331-334.

Furedy, J.J. (1975). An integrative progress report on information control in humans: Some laboratory findings and methodological claims. Australian Journal of Psychology, 27, 61-83.

Furedy, J.J. (1992). Reflections on human Pavlovian decelerative heart-rate conditioning with negative tilt as US: Alternative approaches. Integrative Physiological and Behavioral Science, 27, 347-355.

Furedy, J.J., \& Riley, D.M. (1987). Human Pavlovian autonomic conditioning and the cognitive paradigm. In G. Davey (Ed.), Cognitive processes and Pavlovian conditioning in humans (pp. 1-25). Chichester: John Wiley \& Sons Lid.

Furedy, J.J., \& Schiffmann, K. (1973). Concurrent measurement of autonomic and cognitive processes in a test of the traditional discriminative control procedure for Pavlovian electrodermal conditioning. Journal of Experimental Psychology, 100, 210-217.

Gigerenzer, G. (1991). How to make cognitive illusions disappear: Beyond "heuristics and biases. European Review of Social Psychology, 2, 91-115.

Golding, S.L., \& Rorer, L.G. (1972). Illusory correlation and subjective judgment. Journal of Abnormal Psychology, 80, 249-260.

Gordon, I.E. (1989). Theories of visual perception. Chichester: Wiley.

Graham, F.K. (1979). Distinguishing among orienting, defense, and startle reflexes. In H.D. Kimmel, E.H. van Olst, \& J.F. Orlebeke (Eds.). The orienting reflex in humans (pp. 137-169). New York: Lawrence Erlbaum Publishers.

Grant, D.A. (1968). Adding communication to the signalling property of the CS in classical conditioning. Journal of General Psychology, 79, 141-175.

Gregory, R.L. (1974). Perceptions as hypotheses. In S.C. Brown (Ed.), Philosophy of Psychology. London: MacMillan.

Grings, W.W. (1969). Anticipatory and preparatory electrodermal behavior in paired stimulation situations. Psychophysiology, 5, 597-611. 
Grings, W.W., \& Sukoneck, H.I. (1971). Prediction probability as a determiner of anticipatory and preparatory electrodermal behavior. Journal of Experimental Psychology, 91, 310-317.

Hamilton, D.L., Dugan, P.M., \& Trolier, T.K. (1985). The formation of stereotypic beliefs: Further evidence for distinctiveness-based illusory correlations. Journal of Personality and Social Psychology, 48, 5-17.

Hamilton, D.L., \& Gifford, R.K. (1976). Illusory Correlation in interpersonal perception: A cognitive basis of stereotypic judgments. Journal of Experimental and Social Psychology, 12, 392-407.

Hamm, A. (1992). Modulation protektiver Reflexe und Konditionierung autonomer Reaktionen: Indikatoren kognitiver und affektiver Prozesse beim Klassischen Konditionieren. Paper presented at the 34. Tagung der experimentell arbeitender Psychologen, Osnabrück (Abstract p. 140).

Hamm, A.O., Greenwald, M.K., Bradley, M.M., Cuthbert, B.N., \& Lang, P.J. (1991). The fear potentiated startle effect: Blink reflex modulation as a result of classical aversive conditioning. Integrative Physiological and Behavioral Sciences, 26, 119-126.

Hamm, A. \& Stark, R. (1993) Sensitization and aversive conditioning: Effects on the startle reflex and electrodermal conditioning. Integrative Physiological and Behavioral Science, 28, 171-176.

Hamm, A.O., Vaitl, D., \& Lang, P.J. (1989). Fear conditioning, meaning, and belongingness: A selective association analysis. Journal of Abnormal Psychology, 98, 395-406.

Hawton, K., Salkovskis, P.M., Kirk, J., \& Clark, D.M. (1989). Cognitive therapy for psychiatric problems. Oxford: Oxford University Press.

Hendersen, R.W. (1985). Fearful memories: The motivational significance of forgetting. In F.R. Brush \& J.B. Overmier (Eds.). Affect, Conditioning, and Cognition: Essays on the Determinants of Behavior. Hillsdale NJ: Erlbaum, p. 43-53.

Houtz, J.C. \& Frankel, A.D. (1988). Hemisphericity and problem-solving ability. Perceptual and Motor Skills, 66, 771-774.

Hugdahl, K. (1987).Pavlovian conditioning and hemispheric assymmetry: A perspective. In G.C.L. Davey (Ed.). Cognitive Processes and Pavlovian Conditioning in Humans. (pp. 147-182) Chichester: Wiley.

Hugdahl, K. (1989). Bilateral electrodermal asymmetry: Past hopes and future prospects. International Journal of Neuroscience, 39, 33-44.

Hugdahl, K., Franzon, M., Andersson, B., \& Walldebo, G. (1983). Heart rate responses to lateralized visual stimuli. Pavlovian Journal of Biological Science, 18, 186-198.

Hughdahl, K. \& Johnsen, B. (1989). Preparedness and electrodermal fear-conditioning: ontogenetic vs. phylogenetic explanations. Behaviour Research and Therapy, 27, 269-278.

Hugdahl, K. \& Johnsen, B.H. (1991). Brain asymmetry and human electrodermal conditioning. Integrative Physiological and Behavioral Science, 26, 39-44.

Hull, C.L. (1943). Principles of Behavior. New York: Appleton Century Crofts.

James, W. (1890). Principles of Psychology. New York: Holt.

Johnsen, B.H., \& Hugdahl, K. (1993). Right hemisphere representation of autonomic conditioning to facial emotional expressions. Psychophysiology, 30, 274278. 
Kamin, L.J. (1969). Predictability, surprise, attention, and conditioning. In B.A. Campbell \& R.M. Church (Eds.), Punishment and aversivie behavior. New York: Appleton Century Crofts.

Kelly, H.H. (1973). The process of causal attribution. American Psychologist, 28, 107-128.

Kelly, H.H., \& Michela, J.L. (1980). Attribution theory and research. Annual Review of Psychology 31, 457-501.

Kendall, P.C. (1978). Anxiety: States, trait-situations? Journal of Consulting and Clinical Psychology, 46, 280-287.

Kimmel, H.D. (1966). Inhibition of the unconditioned response in classical conditioning. Psychological Review, 73, 232-240.

Klorman, R., Weerts, T.C., Hastings, J.E., Melamed, B.G., \& Lang, P.J. (1974). Psychometric description of some specific fear questionnaires. Behavior Therapy, 5, 401-409.

Lang, P.J. (1985). The cognitive psychophysiology of emotion: Fear and anxiety. In A.H. Tuma \& J.D. Maser (Eds.), Anxiety and the Anxiety Disorders (pp. 131-170). Hillsdale, NJ: Erlbaum.

Lang, P.J., Melamed, B.G., \& Hart, J. (1970). A psychophysiological analysis of fear modification using an automated desensitization procedure. Journal of Abnormal Psychology, 76, 220-234.

Lang, P.J., Bradley, M.M., \& Cuthbert, B.N. (1990). Emotion, attention, and the startle reflex. Psychological Review, 97, 377-395.

Lang, P.J., Öhman, A., \& Vaitl, D. (1988). The international affective picture system [photographic slides]. Gaineville, FL: Center for Research in Psychophysiology, University of Florida.

Langer, E.J. \& Roth, J. (1975). Heads I win, tails it's chance: The illusion of control as a function of the sequence of outcomes in a purely chance task. Journal of Personality and Social Psychology, 32, 951-955.

Lavy, E. (1993). Attentional bias and anxiety: Conceptual issues and empirical data. PhD thesis, Limburg University, Maastricht.

Lavy, E., van den Hout M., \& Arntz A. (1992). Attentional bias and spider phobia: Conceptual and clinical issues. Behaviour Research and Therapy, 31, 17-24.

Leary, M.R., Britt, T.W., Cutlip, W.D., Templeton, J.L. (1992). Social blushing. Psychogical Bulletin, 112, 446-460.

Lennox, S.S., Bedell, J.R., Abramson, L.Y., Raps, C., et al. (1990). Judgment of contingency: A replication with hospitalized depressed, schizophrenic, and normal samples. Journal of Social Behavior and Personality, 5, 189-204.

Logan, A.C., \& Goetsch, V.L. (1993). Attention to external threat cues in anxiety states. Clinical Psychology Review, 13, 541-559.

Lousberg, R. (1993). Chronic pain: Behavioural diagnostics and mechanisms. PhD thesis, Limburg University, Maastricht (chapters 4 \& 8).

Lovibond, P.F. (1988). Predictive validity in human causal judgement and Pavlovian conditioning. Biological Psychology, 27, 79-93.

Lykken, D.T. (1972). Range correction applied to heart rate and GSR data. Psychophysiology, 9, 373-382.

Lykken, D.T., Macindoe, I., and Tellegen, A. (1972). Preception: Autonomic response to shock as a function of predictability in time and locus. Psychophysiology, 9, 318-333.

Mackie, D.M., Hamilton, D.L., Schroth. H.A., Carlisle, C.J., Gersho, B.F., 
Meneses, L.M., Nedler, B.F., \& Reichel, L.D. (1989). The effects of induced mood on expectancy-based illusory correlations. Journal of Experimental Social Psychology, 25, 524-544.

MacLeod, C., \& Hagan, R. (1992). Individual differences in the selective processing of threatening information, and emotional responses to stressful life event. Behaviour Research and Therapy, 30, 151-161.

MacLeod, C., \& Mathews, A. (1991). Biases cognitive operations in anxiety: Accessibility of information or assignment of processing priorities? Behaviour Research and Therapy, 29, 599-610.

Maltzman, I. (1987). A neo-Pavlovian interpretation of the OR and classical conditioning in humans: With comments on alcoholism and the poverty of cognitive psychology. In G. Davey (Ed.) Cognitive processes and Pavlovian Conditioning in Humans (pp 211-249). New York: Wiley.

Margraf, J. \& Schneider S. (1993). Psychological treatment of panic: what works on the long run. Abstract in the proceedings of the 23rd European Congress of Behaviour and Cognitive Therapies. London, September (p.15-16).

Marks, I. (1977). Phobias and obsessions. Clinical phenomena in search of laboratory models. In J.D. Maser \& M.E.P. Seligman (Eds.). Psychopathology: Experimental Models. San Fransisco: Freeman.

Marks, I.M. \& Mathews, A.M. (1979). Brief standard self-rating for phobic patients. Behaviour Research and Therapy, 17, 263-267.

Mathews, A., \& MacLeod, C. (1985). Selective processing of threat cues in anxiety states. Behaviour Research and Therapy, 23, 563-569.

McArthur, L.Z., \& Friedman, S.A. (1980). Illusory correlation in impression formation: Variations in the shared distinctiveness effect as a function of the distinctive person's age, race, and sex. Journal of Personality and Social Psychology, 39, 615-624.

McNally, R.J. \& Foa, E.B. (1987). Cognition and agoraphobia: bias in the interpretation of threat. Cognitive Therapy and Research, 11, 567-581.

McNally, R.J., \& Heatherton, T.F. (1993). Are covariation biases attributable to a priori expectancy biases? Behaviour Research and Therapy, 31, 653-658.

Merckelbach, H., Arrindell, W.A., Arntz, A., \& de Jong, P.J (1992). Pathways to spider phobia. Behaviour Research and Therapy, 30, 543-546.

Merckelbach, H, \& de Jong, P.J. (1988). Conditioned inhibition of psychophysiological responses to painful stimuli: Facts and suggestions. Psychologica Belgica, 28, 139-155.

Merckelbach, H., Arntz, A., \& de Jong, P.J. (1990). Conditioning experiences in spider phobics. Behaviour Research and Therapy, 29, 333-335.

Merckelbach, H., de Jong, P.J., \& Arntz, A. (1991). Imagery ability and exposure in vivo in spider phobia. Behaviour Research and Therapy, 29, 203-205.

Merckelbach, H., de Ruiter, C., van den Hout, M.A., \& Hoekstra, R. (1989). Conditioning experiences and phobias. Behaviour Research and Therapy, 27, 657-662.

Merckelbach, H., Muris, P., \& de Jong, P.J. (1990) Hemisphere preference, phobia, and depression. International Journal of Neuroscience, 55, 119-123.

Merckelbach, H. \& van Oppen, P. (1989). Effects of gaze manipulation on subjective evaluation of neutral and phobia-relevant stimuli: A comment on Drake. Acta Psychologica, 70, 1-5.

Merckelbach, H. \& van den Hout, M.A. (1991). Fear relevance and diminution of 
unconditioned skin conductance responses. Zeitschrift für Psychologie, 199, 267-277.

Merckelbach, H., van den Hout, M.A., \& de Jong, P. (1989). Psychometric and electrodermal aspects of illusory correlations: Paradoxal results. Psychologica Belgica, 29, 135-148.

Merckelbach, H., Van Hout, W., de Jong, P.J., \& van den Hout, M.A. (1990). Classical conditioning and attentional bias. Journal of Behavior Therapy and Experimental Psychiatry, 21, 185-191.

Miller, S.M. (1987). Monitoring and blunting: Validation of a questionnaire to assess styles of information seeking under threat. Journal of Personality and Social Psychology, 52, 345-353.

Mineka, S. \& Sutton, S.K. (1992). Cognitive biases and emotional disorders. Psychological Science, 3, 65-69.

Mineka, S. \& Tomarken, A.J. (1989). The role of cognitive biases in the origens and maintenance of fear and anxiety disorders. In T. Archer \& L-G. Nilsson (Eds.). Aversion, Avoidance, and Anxiety: Perspectives on Aversily Motivated Behavior (pp 195-221). Hillsdale, NJ: Erlbaum.

Morrow, M.C. (1966). Recovery of conditioned UCR diminution following extinction. Journal of Experimental Psychology, 74, 884-888.

Muris, P. \& de Jong, P.J. (1993). Monitoring and perception of threat. Personality and Individual Differences, 15, 467-470.

Muris, P., de Jong, P.J., Merckelbach, H., \& van Zuuren, F. (1993). Effects of imposed monitoring and blunting strategies on emotional reactivity. Anxiety, Stress, and Coping, in press.

Muris, P., de Jong, P.J., Merckelbach, H., \& van Zuuren, F. (1994). Monitoring coping style and exposure outcome in spider phobics. Behavioural and Cognitive Psychotherapy, 21, 329-333.

Murray, E.J., \& Foote, F. (1979). The origens of fear of snakes. Behaviour Research and Therapy, 17, 489-493.

Newlin, D.B. (1989). The skin-flushing response: Autonomic, self-report, and conditioned responses to repeated administrations of alcohol in Asian men. Journal of Abnormal Psychology, 98, 421-425.

Nisbett, R.E., Fong, G.T., Lehman, D.R., \& Cheng, P.W. (1987). Teaching reasoning. Science, 238, 625-631.

Nisbett, R., \& Ross, L. (1980). Human Inference: Strategies and Shortcommings of Social Judgment. Englewood Cliffs NJ: Prentice-Hall.

Nisbett, R.E. \& Wilson, T.D. (1977). Telling more than we can know: Verbal reports on mental processes. Psychological Review, 84, 231-259.

Oexle, J.E. \& Zenhausern, R. (1981). Differential hemispheric activation in good and poor readers. International Journal of Neuroscience, 15, 31-36.

Öhman, A. (1979). The orienting response, attention, and learning: An information processing perspective. In H.D. Kimmel, E.H. Van Olst, \& J.F. Orlebeke (Eds.), The orienting reflex in humans. Hillsdale, NJ: Erlbaum.

Öhman, A., Fredrikson, M., Hugdahl, K., \& Rimmo, P-A. (1976). The premise of equipotentiality in human classical conditioning: Conditioned electrodermal responses to potentially phobic stimuli. Journal of Experimental Psychology: General, 105, 313-337.

Papini, M.R., \& Bitterman, M.E. (1990). The role of contingency in classical conditioning. Psychological Review, 97, 396-403. 
experimentation. American Journal of Psychology, 28, 163-174.

Watson, J.P., \& Marks, I.M. (1971). Relevant and irrelevant fear in flooding: A crossover study of phobic patients. Behavior Therapy, 2, 275-293.

Watts F.N., McKenna F.P., Sharrock R., \& Trezise L. (1986). Colour naming of phobia-related words. British Journal of Psychology, 77, 97-108.

Weber, S.L. \& Sackheim, H.A. (1984). The development of functional brain asymmetry in the regulation of emotion. In N.A. Fox \& R.J. Davidson (Eds.). The psychobiology of affective development. Hillsdale NJ: Erlbaum.

Weiner, B. (1980). Human motivation. New York: Holt Rinehart \& Winston.

Weiner, B. (1985). "Spontaneous" causal thinking. Psychological Bulletin, 97, 7484.

White, K. \& Davey, G.C.L. (1989). Sensory preconditioning and UCS inflation in human fear conditioning. Behaviour Research and Therapy, 27, 161-166.

Williams, J.M.G., Watts, F.N., MacLeod, C., \& Mathews, A. (1988). Cognitive Psychology and Emotional Disorders. New York: John Wiley \& Sons.

Wolff, P.H. (1972). Vasomotor sensitivity to alcohol in diverse Monoloid populations. American Journal of Human Genetics, 25, 193-199.

Yates, J.F. \& Curley, S.P. (1986). Contingency judgment: Primacy effects and attention decrement. Acta Psychologica, 62, 293-302.

Zenhausern, R. (1978). Imagery, cerebral dominance, and style of thinking: A unified field model. Bulletin of the Psychonomic Society, 12, 381-384.

Zenhausern, R., Notaro, J., Grosso, J., \& Schiano, P. (1981). The interaction of hemispheric preference, laterality and sex in the perception of emotional tone and verbal content. International Journal of Neuroscience, 13, 121-126.

Zillman, D. (1983). Transfer of excitation in emotional behavior. In J.T. Cacioppo \& R.E. Petty (Eds.), Social Psychophysiology (pp. 215-235), NY: Guilford Press. 
In dit proefschrift staan een aantal mechanismen centraal die mogelijkerwijs een rol spelen bij het ontstaan en voortbestaan van fobische angst. Met betrekking tot angststoornissen is klassieke conditionering nog altijd één van de meest invloedrijke theorieën. In zijn meest elementaire vorm wordt binnen dergelijke theorieën het ontstaan van pathologische angst toegeschreven aan de koppeling van voorheen neutrale stimuli (b.v. een auto) aan een traumatische ervaring (b.v. een ongeluk), een min of meer automatisch proces.

Vanuit een dergelijk leertheoretisch perspectief kunnen angstwekkende stimuli (liften voor claustrofobici, hartkloppingen voor paniek patiënten, spinnen voor spinfobici) worden beschouwd als voorspellers voor aversieve (en dus ongewenste) gebeurtenissen. In het geval angstpatiënten dergelijke stimuli inderdaad beschouwen als voorspellers voor naderend onheil, is het niet verbazingwekkend dat zij de bewuste stimuli zoveel mogelijk trachten te vermijden. Vermijding lijkt hier tenslotte een passende strategie om de kans op ongewenste gebeurtenissen te minimaliseren. Echter, het is evident dat een dergelijke sequentiële relatie tussen gevreesde stimuli en aversieve gebeurtenissen in werkelijkheid niet bestaat: liften storten zelden neer, spinnen plegen geen letsel toe te brengen, en hartkloppingen worden als regel niet gevolgd door een infarct. Deze paradox roept de vraag op waarom angstpatiënten desalniettemin voortdurend handelen alsof zo'n relatie wel degelijk bestaat.

\section{Illusoire Correlaties}

In een poging om licht te werpen op deze kwestie wordt in hoofdstuk 2 de suggestie getoetst dat ten gevolge van pathologische angst mensen ertoe neigen de samenhang tussen gevreesde stimuli en aversieve gebeurtenissen te overschatten. Met andere woorden dat ze illusoire verbanden waarnemen als gevolg van een zogenaamde 'covariation bias'. Dit zou bij angstpatiënten resulteren in een over-representatie van vreeswekkende stimuli en contingente aversieve gebeurtenissen. Anecdotes van spinfobici die bij ons meededen aan een training tegen hun fobie, laten zien dat dergelijke representaties van relaties drastisch kunnen afwijken van de realiteit. $\mathrm{Zo}$ is een veel gehoorde waarneming dat spinnen altijd op de betrokken personen afrennen, terwijl ze in werkelijkheid natuurlijk minstens zo vaak een andere kant uitschieten; een mevrouw vertelde me het fantastische verhaal dat spinnen haar huis overdag bezochten als haar man in de dagdienst zat, maar juist 's nachts wanneer hij nachtdienst had...

De experimenten in hoofdstuk 2 bevestigen de boven beschreven suggestie dat fobici de neiging vertonen illusoire correlaties waar te nemen tussen fobie-relevante stimuli en aversieve prikkels. De belangrijkste implicatie van deze resultaten is dat fobici kennelijk inderdaad informatie op een zodanige wijze verwerken, dat het hun angst bevestigt en mogelijk zelfs versterkt. $\mathrm{Nu}$ is wel tegengeworpen dat cognitieve bias slechts een bijver- 
schijnsel zou zijn van fobische angst, een epifenomeen zonder causale status. En het moet worden erkend, de bevinding dat angstige mensen een covariation bias vertonen terwijl de bias afwezig is na succesvolle behandeling, sluit niet uit dat zo'n bias slechts een symptoom is van angst (ofschoon het eveneens prima past binnen de eerder gesuggereerde reciproke relatie tussen angst en covariation bias). Hoofdstuk 4 maakt echter aannemelijk dat cognitieve bias niet louter een epifenomeen is maar dat er wel degelijk een reciproke relatie bestaat tussen angst en bias: succesvol behandelde spinfobici met een residuele covariation bias vertoonden 2 jaar na behandeling een grotere terugval dan de fobici die direct na behandeling geen bias meer vertoonden. Bijelkaar genomen lijkt het redelijk om te veronderstellen dat covariation bias het bedreigende karakter van fobische stimuli intensiveert, hetgeen op zijn beurt de kans vergroot dat de angst voor die stimuli (weer) wordt aangewakkerd. Covariation bias zou aldus weleens een cruciale rol kunnen spelen bij het ontstaan en de instandhouding van fobische angst.

In hoofdstuk 3 worden een aantal routes beschreven waarlangs zo'n covariation bias kan onstaan. Hoofdstuk 3a laat zien dat incidentele coincidenties van (voorheen) neutrale stimuli en aversieve prikkels kan leiden. tot het waarnemen van illusoire verbanden. Opmerkelijk genoeg bleek een dergelijke illusoire correlatie bestand tegen een uitdovingsprocedure; sterker nog, ondanks non-contingente aanbieding van de stimuli en de aversieve prikkels nam de illusoire correlatie alleen maar toe. Kennelijk is een eenmaal geïnduceerde covariation bias "self-supporting". In de hoofdstukken $3 b$ en $3 c$ staat de rol van verwachtingen bij het waarnemen van illusoire correlaties centraal. Kortweg suggereren deze hoofdstukken dat het waarnemen van illusoire samnhangen voortvloeit uit verwachtingen die ongevoelig zijn voor disconfirmerende informatie. Op hun beurt kunnen die verwachtingen worden geïnduceerd door conditionerende ervaringen, fobische angst en culturele connotatie.

Hoofdstuk $3 \mathrm{~d}$ beoogt de neuropsychologische verankering van covariation bias te exploreren. Recent onderzoek laat niet alleen zien dat angstige mensen zich kenmerken door cognitieve bias, angstige mensen lijken zich eveneens van niet-angstige mensen te onderscheiden op grond van hun "hemisfeer preferentie". Personen die hoog scoren op allerlei klinisch relevante indices van angst blijken een voorkeur te hebben voor cognitieve processen die verondersteld worden kenmerkend te zijn voor de rechter hemisfeer. Ook studies waarin hemisfeer activatie direct is gemeten ondersteunen de gedachte dat vooral de rechter hemisfeer betrokken is bij de mediatie van angst. Het is niet alleen aannemelijk gemaakt dat negatieve emoties samengaan met relatief sterke rechter hemisfeer activiteit, het is eveneens aangetoond dat de drempel voor het ervaren en uiten van angst is verlaagd bij mensen die habitueel een relatief sterke rechter hemisfeer activiteit vertonen. Anders gezegd, naast de relatie tussen 1.) angst en cognitieve bias en de relatie tussen 2.) angst en hemisfericiteit lijkt er tevens een relatie te bestaan tussen 3.) hemisfericiteit en cognitieve bias. Dit zou er op kunnen duiden dat de relatie tussen angst en cognitieve bias wordt gemediëerd door hemisfeer preferentie (bv. via de verschillende cognitieve karakteristieken die kunnen worden toegeschreven aan 
beide hemisferen). Volgens deze hypothese zou een relatief sterke rechter hemisfeer activatie het optreden van cognitieve bias in de hand werken. Ofschoon onderhavig onderzoek de relatie tussen hemisfeer preferentie en angst bevestigt, levert het voor de relatie tussen hemisfericiteit en covariation bias slechts voorlopige ondersteuning op.

\section{UCS-Inflatie}

Hoofdstuk 5 richt zich op een observatie die problematisch lijkt voor een leertheoretische verklaring van het ontstaan van fobische angst; namelijk de observatie dat een groot aandeel van de mensen die een zogenaamde traumatische ervaring hebben meegemaakt (b.v. ernstig toegetakeld door een hond) geen fobie ontwikkelt, terwijl tegelijkertijd een fors percentage van de mensen die wel een fobie hebben ontwikkelt zich geen traumatische ervaring met het fobisch object kan herinneren. Deze combinatie van bevindingen lijkt op het eerste oog fataal voor een opvatting die zegt dat een fobie onstaat door de koppeling van een aversieve gebeurtenis (UCS) aan een voorheen neutrale stimulus (CS). De vraag rijst of er nog wel een conditioneringstheorie over fobische angst is te bedenken die dergelijke bevindingen kan verdisconteren.

Een variant waarvoor het gesignaleerde probleem in principe geen onoverkomelijk obstakel hoeft te vormen is de benadering van Graham Davey. Het begrip UCS-representatie staat centraal in zijn opvatting. Tengevolge van een CS/UCS koppeling roept de CS een geheugenrepresentatie op van de aversieve gebeurtenis; deze representatie roept vervolgens op haar beurt de geconditioneerde respons (CR) op. Dus de CS lokt niet rechtstreeks een CR uit maar via de UCS-representatie. Een belangrijke implicatie van deze stellingname is dat alles wat de geheugenrepresentatie beinvloedt repercussies heeft voor de CR. In zijn model zijn twee afzonderlijke fases te onderscheiden in het conditioneringsproces dat leidt tot fobische angst. De eerste fase heeft betrekking op de CS/UCS koppeling; een hond (CS) die bijt (UCS); een akelige behandeling (UCS) bij de tandarts (CS). Fobici zullen deze fase gemeen hebben met vele niet angstige personen; het moet benadrukt dat zolang de representatie zwak is, er geen (of slechts een milde) CR zal optreden. De tweede fase omvat gebeurtenissen (al dan niet contingent met de CS) die leiden tot een toename van de aversiviteit van de UCS-geheugenrepresentatie (b.v. de persoon verneemt dat iemand dood gaat aan de verwondingen van een hondebeet). Door de CS/UCS koppeling in de eerste fase zal de CS na intensivering van de UCS representatie (tweede fase; "UCS-inflation") een fobische respons gaan uitlokken. Dus volgens dit model kan het onvermogen van sommige fobici om een conditionerende ervaring te rapporteren, voortvloeien uit het feit dat de aversiviteit van de UCS-geheugenrepresentatie pas in een tweede fase (in afwezigheid van de CS) fobische proporties heeft verkregen.

Het model van Davey kan eveneens uit de voeten met de bevinding dat sommige mensen die wel degelijk een conditionerende ervaring hebben 
meegemaakt desalniettemin geen fobie ontwikkelen. Dergelijke gevallen kunnen worden begrepen door gebeurtenissen te veronderstellen die de aversiviteit van de UCS-geheugenrepresentatie juist hebben verlaagd ("UCSdeflation"). In het verlengde van deze visie kan therapie dan ook worden beschouwd als een massieve poging tot "UCS-deflation".

Om de houdbaarheid van het UCS-inflatie model te toetsen, is in eerste instantie onderzocht of het überhaupt mogelijk is casuistiek te vinden waarbij het ontstaan van de angstklachten kan worden geformuleerd volgens een UCSinflatie scenario. Ervaren clinici die wij wij hiertoe uitnodigden kwamen met sprekende voorbeelden (zie hoofdstuk 5a.). Het moet echter worden benadrukt dat de mogelijkheid om de etiologische route van bepaalde casus post hoc te beschrijven volgens een UCS-inflatie scenario nog niet impliceert dat dergelijke processen inderdaad een rol hebben gespeeld bij het ontstaan van de klachten. Ook al spreken sommige voorbeelden tot de verbeelding, ze zijn vrijblijvend zolang een degelijke experimentele onderbouwing van het model ontbreekt.

Om de houdbaarheid van het UCS inflatie model nader te onderzoeken is derhalve een reeks experimenten uitgevoerd die beschreven staan in hoofdstuk 5. De resultaten van hoofdstuk 5 ondersteunen de suggestie dat mensen ontvankelijk zijn voor UCS-inflatie procedures. Het moet echter worden benadrukt dat uitsluitend onder zeer strikte laboratorium condities evidentie kon worden gevonden voor de invloed van UCS-inflatie op de CR. Naarmate de experimenten de klinische praktijk dichter naderden, werden de resultaten zwakker. Terzijde zij nog opgemerkt dat hoofdstuk $5 b$ geen evidentie opleverde voor een interactie tussen persoonlijkheidskenmerken en UCS inflatie procedures.

\section{De Oogknipperreflex}

Waar in de hoofdstukken $2 \mathrm{t} / \mathrm{m} 5$ de nadruk ligt op factoren die van belang zijn bij het ontstaan en de instandhouding van fobische angst, beschrijft hoofdstuk 6 onderzoek naar indices die het resultaat van behandeling kunnen voorspellen. Centraal in dit hoofdstuk staat een relatief nieuwe fysiologische index van emoties: de oogknipperreflex. Recent onderzoek heeft aangetoond dat in het geval de algehele toestand van een persoon is gericht op toenadering (bijvoorbeeld bij het zien van een smakelijke vlaai) een plots hard geluid slechts een relatief kleine oogknipperreflex induceert. Wanneer de toestand echter gericht is op vermijding (bijvoorbeeld als het oog valt op een pitbullterrier) blijkt een zelfde geluid een veel grotere reflex uit te lokken. Op grond van deze resultaten is wel gesuggereerd dat de oogknipperreflex een bruikbare index zou zijn voor de uitkomst en prognose van therapie.

In de studies beschreven in hoofdstuk $6 \mathrm{a}$ en $6 \mathrm{~b}$ wordt deze suggestie bij een groep spinfobici experimenteel getoetst. Conform de verwachting waren de oogknipper-responsen van spinfobici krachtiger tijdens de confrontatie met een spin dan tijdens de confrontatie met een mandje met etenswaren. Na 
afloop van behandeling bleken de schrikreacties tijdens de aanwezigheid van dezelfde spin significant te zijn afgenomen. De uitgelokte schrikreacties tijdens de meting met het mandje lekkernij bleven daarentegen van gelijke grootte. De bevinding dat de uitgelokte knipper-reflex kan worden afgezwakt door therapie, wijst erop dat de respons bruikbaar is als (additionele) therapieuitkomst maat.

Een ander opmerkelijk resultaat was, dat de uitgelokte schrikreaktie als enige van de gebruikte variabelen voorspellende waarde had voor het (korte em lange termijn) therapiesucces. Mensen die voor behandeling relatief sterke uitgelokte oogknipper-responsen vertoonden tijdens de gedragsmeting, durfden na afloop van de behandeling een spin dichter te naderen, dan fobici die vooraf relatief zwakke schrikreakties lieten zien (hoofdstuk 6a).

Hoofdstuk $6 \mathrm{~b}$ biedt vervolgens experimentele evidentie voor de stellingname dat de oogknipperreflex (ook) in de context van een gedragsmeting met name gevoelig is voor de emotionele richting (vermijding vs. naderen) en in veel mindere mate voor arousal. Bovendien laat dit hoofdstuk zien dat de zelfgerapporteerde schrikreacties nauw gerelateerd zijn aan de grootte van de oogknipperreflex. Deze relatie biedt de mogelijkheid uitgelokte schrikreacties ook buiten het laboratorium (bijvoorbeeld in klinische setting) te gcbruiken. Afsluitend kan worden gesteld dat de gepresenteerde bevindingen bepaald hoopvol zijn: de oogknipper-reflex lijkt niet alleen een bijdrage tc kunnen leveren als therapie-uitkomst maat, maar onder bepaalde omstandigheden ook als voorspeller van therapiesucces. Nu hadden onze gegevens betrekking op mensen met een geïsoleerde angstklacht. Het valt te bezien of de knipperreflex ook een bruikbaar instrument is bij het in kaart brengen van behandelingseffecten bij complexe angststoornissen (agorafobie; dwangneurose, etc.). 


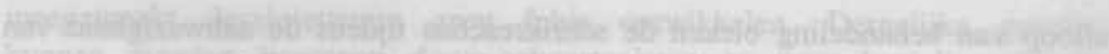

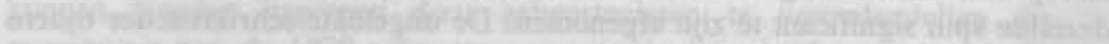

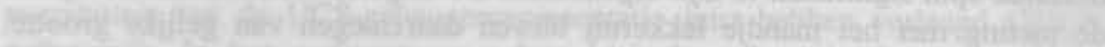

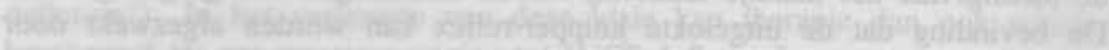

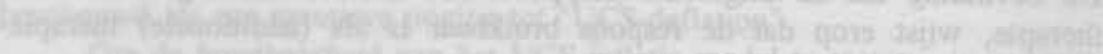

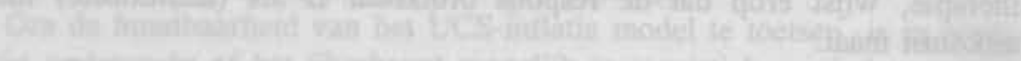

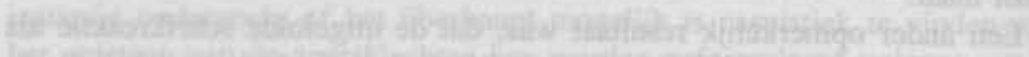

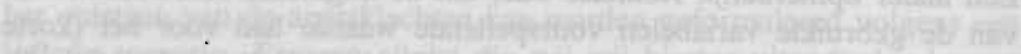

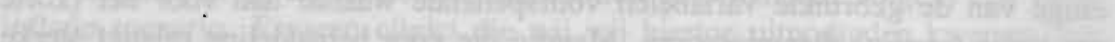
(15)

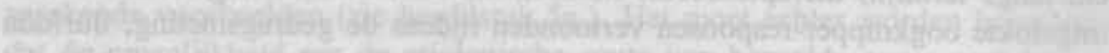

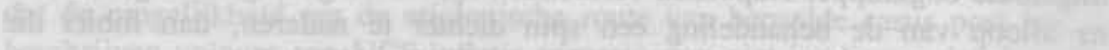

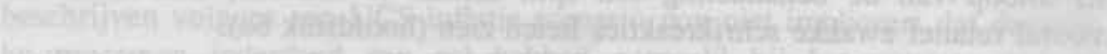

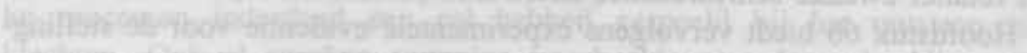

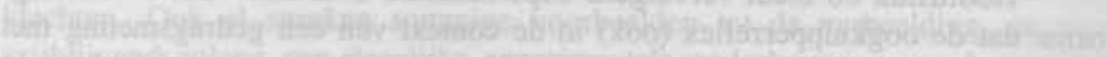

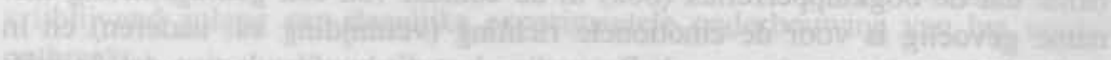

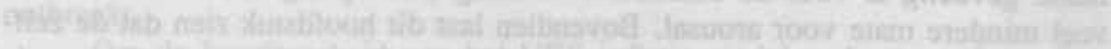

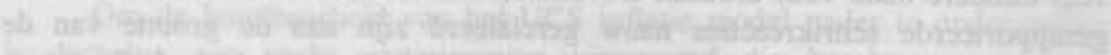

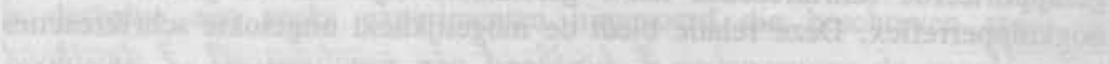

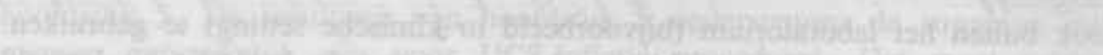

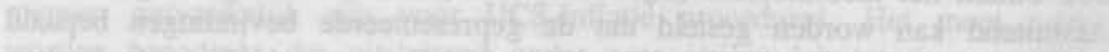

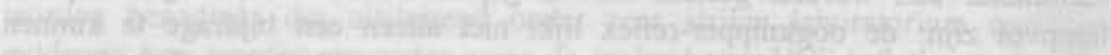

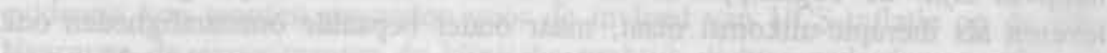

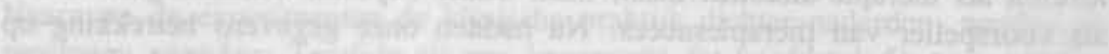

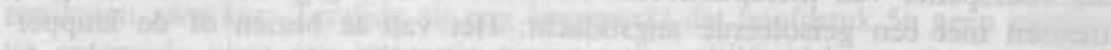

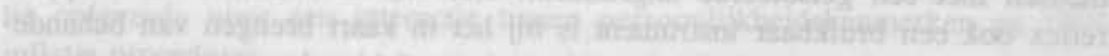

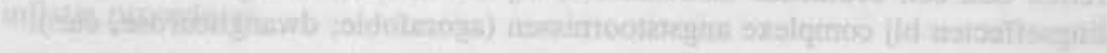

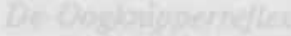

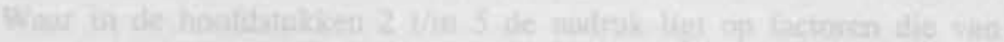

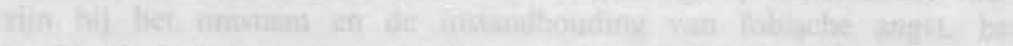

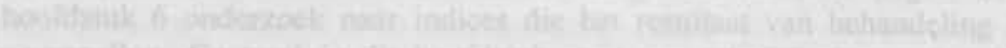

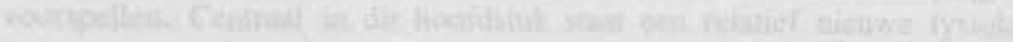

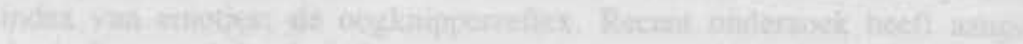

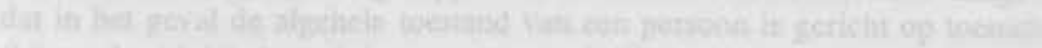

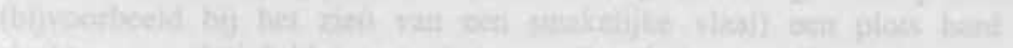

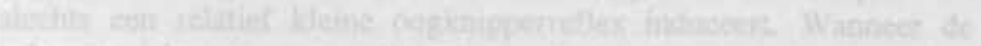

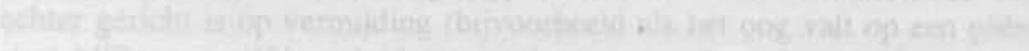

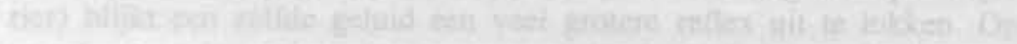

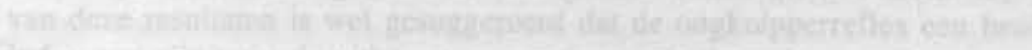

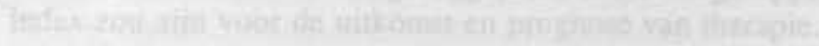

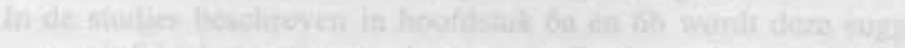

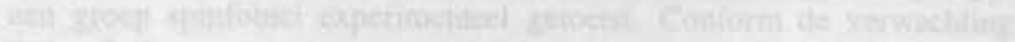

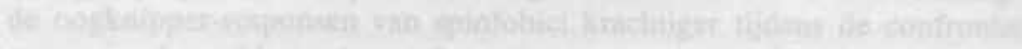

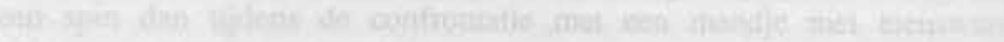




\section{DANKWOORD}

Voor de totstandkoming van het proefschrift ben ik in de eerste plaats veel dank verschuldigd aan Harald Merckelbach. Steeds nadrukkelijker realiseer ik mij hoezeer ik geboft heb met zo'n begeleider. Het was (is) heel prettig met je samenwerken; altijd bereikbaar voor discussie, immer stimulerende kritiek, en altijd op uiterst korte termijn suggesties bij en deskundig commentaar op voorgelegde stukken. Daarnaast heb ik het getroffen met de collega's van het onderzoeksthema Experimentele Psychopathologie. Met name wil ik Arnoud Arntz noemen die ook co-auteur is bij de meeste publicaties die zijn opgenomen in dit proefschrift. Ik heb veel van hem geleerd op het gebied van de experimentele psychologie en statistiek. Graag wil ik ook Marcel van den Hout bedanken die als promotor met name bij de afronding van het proefschrift de belangrijke functie van klankbord heeft vervuld. Daarnaast bewaar ik goede herinneringen aan de samenwerking met Idith Lavy die in dezelfde periode bezig was met haar promotie-onderzoek. Buitengewoon veel plezier heb ik beleefd aan de samenwerking met Peter Muris. Het was (is) inspirerend om samen met jou over onderzoek te brainstormen en experimenten uit te voeren. Eveneens met plezier kijk ik terug op ons gezamenlijk congresbezoek.

Graag wil ik ook alle mensen bedanken die hebben bijgedragen aan het welslagen van de verschillende "spinprojecten" als therapeut, onderzoeksassistent, coördinator en/of technicus. Met name wil noemen Theo van Aerts, Germie van den Berg, Arie Dijkstra, Birgit Mayer, Anja Meijboom, Dorien Wolfs en Gerrie van Wunnik. Dank niet alleen voor de praktische bijdrage maar ook voor de gezelligheid; een voorwaarde om dit soort uitputtende projecten te volbrengen. Tenslotte wil ik de AIO's en bestuursleden van het AIO netwerk Experimentele Psychopathologie bedanken voor de vruchtbare en gezellige bijeenkomsten in het "Witte Huis". Rest mij Erik Schouten en Angela Verweij te bedanken. Erik stond altijd klaar om computers aan de praat te houden en was altijd paraat voor het bespreken van statistische problemen; Angela verzorgde de secretariële ondersteuing van het onderzoeksprogramma EPP. 


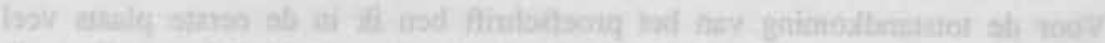

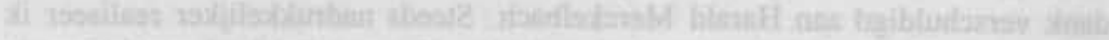

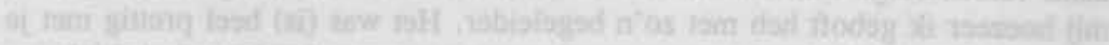

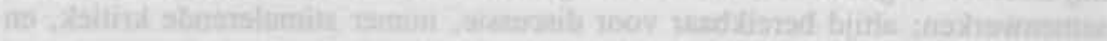

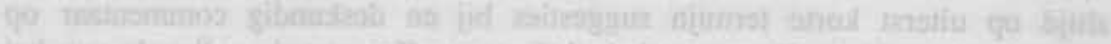

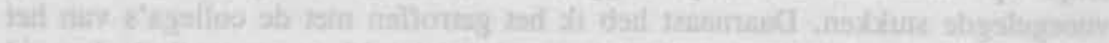

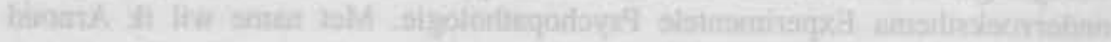

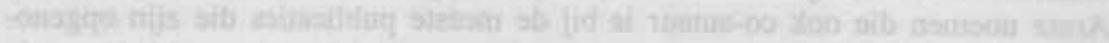

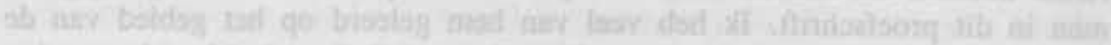

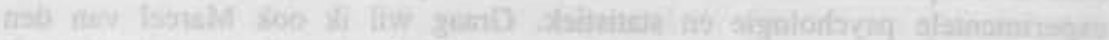

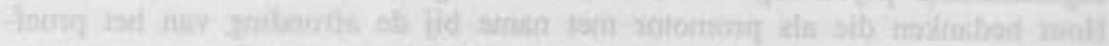

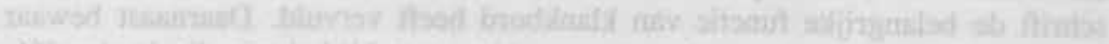

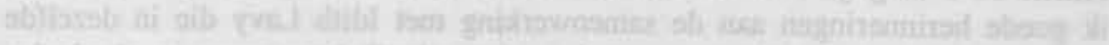

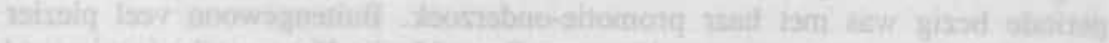

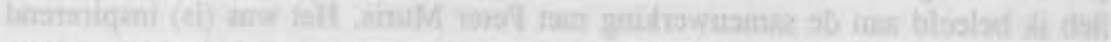

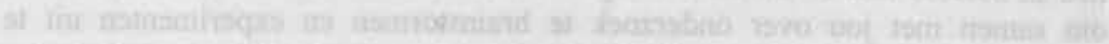

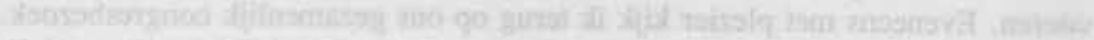

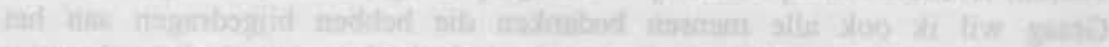

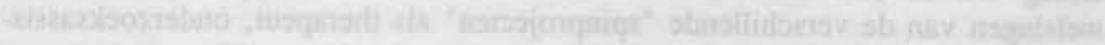

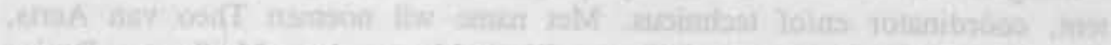

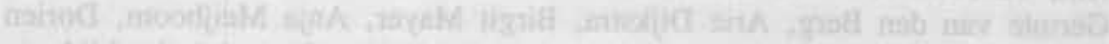

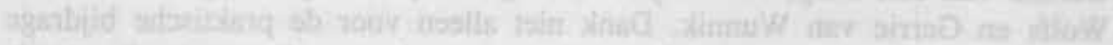

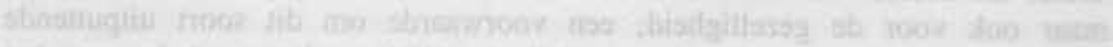

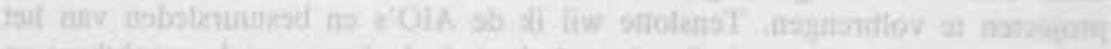

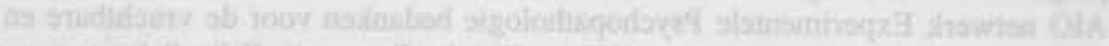

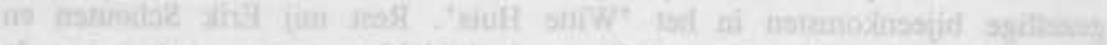

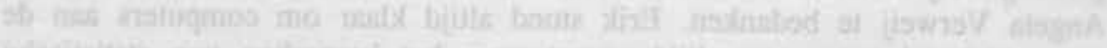

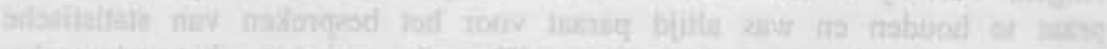

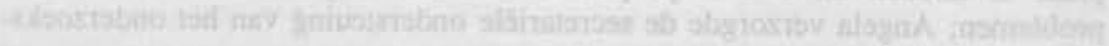




\section{CURRICULUM VITAE}

Peter de Jong werd op 11 februari 1962 te Delft geboren. In 1980 behaalde hij het gymnasium B diploma aan het Ichthus College te Drachten. In datzelfde jaar begon hij zijn studie Lichamelijke Opvoeding (tegenwoordig Bewegingswetenschappen) aan de Vrije Universiteit te Amsterdam. Na het behalen van het kandidaatsexamen Lichamelijke Opvoeding volgde hij het verkort kandidaatsprogramma Wijsbegeerte. In februari 1988 studeerde hij af met als hoofdrichting Inspanningsfysiologie en nevenrichtingen Functionele Anatomie en Psychologie. Tevens heeft hij deelgenomen aan de onderwijskundige opleiding ter verkrijging van de lesgeefbevoegdheid voor de medisch biologische vakken. In aansluiting hierop werd hij in het voorjaar 1987 tijdelijk aangesteld als docent humane biologie bij de lerarenopleiding d'Witte Leli in Amsterdam. Vanaf april 1988 was hij als erkend gewetensbezwaarde militaire dienst werkzaam bij de vakgroep Geestelijke Gezondheidkunde van de Rijksuniversiteit Limburg. Bij dezelfde vakgroep kreeg hij vervolgens in het najaar 1989 een aanstelling als assistent in opleiding. Gedurende deze aanstelling heeft hij het onderzoek verricht dat in dit proefschrift beschreven staat. Sinds oktober 1992 is hij werkzaam als universitair docent bij de vakgroep Differentiële en Experimentele Psychologie van de Rijksuniversiteit Limburg. Als zodanig is hij nauw betrokken bij het onderwijs ten behoeve van de afstudeerrichting Geestelijke Gezondheidkunde en verricht hij onderzoek rond het thema informatieverwerking en psychopathologie binnen de onderzoeksgroep Experimentele Psychopathologie.

\section{List of Publications}

Arntz, A., Dreesen, L., \& de Jong, P. (1994). The influence of anxiety on pain: Attentional and attributional mediators. Pain, 56, 307-314.

Arntz, A., van Eck, M., de Jong, P., \& van den Hout, M.A. (1989). The relationship between underpredicted pain and escape. Behaviour Research and Therapy, 28, 87-90.

Amtz, A., van Eck, \& de Jong, P.J. (1991). Avoidance of pain of unpredictable intensity. Behaviour Research and Therapy, 29, 197-201

Amtz, A., van Eck, M., \& de Jong, P.J. (1992). Unpredictable sudden increase of pain intensity and acquired fear. Journal of Psychophysiology, 6, 54-64

Amtz, A. \& de Jong, P.J. (1992). Anxiety, attention, and pain. Journal of Psychosomatic Research, 37, 423-432.

Arntz, A., Merckelbach, H., \& de Jong, P. (1993). Opoid antagonist affects behavioral effects of exposure in vivo treatment of phobic fear. Joumal of Consulting and Clinical Psychology 61, 865-870.

Davey, G.C.L, de Jong, P.J., \& Tallis, F. (1993). UCS inflation in the aetiology of a variety of anxiety disorders: Some case histories. Behaviour 
Research and Therapy, 31, 495-498.

Van den Hout, M.A., de Jong, P., Zandbergen J., \& Merckelbach, H. (1990)

Waning of panic sensations during prolonged hyperventilation. Behaviour Research and Therapy, 28, 445-448.

Van den Hout, M.A., Jansen A., \& de Jong, P. (1992). Opmerkelijke effecten van erg lang hyperventileren (een experiment). Directieve Therapie, 12, 296-305.

Van den Hout, M.A., de Jong, P. \& Merckelbach, H. (1992). A hyperventilation explanation of the termination of panic attacks; A reply to Ley. Behaviour Research and Therpy, 31, 117-118.

Jansen, A., Gubbens, I., Gransier, S., de Jong, P., Oosterlaan, J., \& Amtz, A. (1989). Is counterregulation of restrained eaters after forced consumption of a preload dependent on mood? Scandinavian Journal of Behaviour Therapy, 18, 119-127.

De Jong, P.J. (1991). International Congress on Anxiety, Stress, and Emotional Disorders. Gedrag en Gezondheid, 19, 282-283.

De Jong, P.J. (1992). Alles over angst. Gedrag en Gezondheid, 20, 311-312.

De Jong, P.J. (1992). Bang zijn en bang blijven: Over fobische angst en het waarnemen van illusoire verbanden. Directieve Therapie, 12, 306-314.

De Jong, P.J. (1993). Covariation bias in phobia: Mere resistance to preexperimental expectancies? Behavior Therapy, 24, 447-454.

De Jong, P.J. (1993). Introduction. In P.J. de Jong (Ed.), Phobia: Etiological, Cognitive, and Physiological Aspects. Advances in Behaviour Research and Therapy, 15, 241-242.

De Jong, P.J. \& Amtz, A. (1993). The role of conditioning in the placebo response. A reply to Voudouris et al. Pain, 54, 232-233.

De Jong, P.J., Arntz, A.,\& Merckelbach, H. (1993). The startle probe response as an instrument for evaluating exposure effects in spider phobia. Advances in Behaviour Research and Therapy, 15, 301-316.

De Jong. P.J. \& Merckelbach, H. (1989). Eyeblink frequency, rehearsal activity and sympathetic arousal. International Journal of Neuroscience, 51, 8994.

De Jong, P.J, \& Merckelbach (1991). Covariation bias and electrodermal responding before and after behavioural treatment. Behaviour Research and Therapy, 29, 307-314.

De Jong, P.J. \& Merckelbach, H. (1992). De oogknipper-reflex: over angst voor spinnen en een veelbelovende nieuwe index voor emoties. De Psycholoog, 27. 148-152.

De Jong, P.J. \& Merckelbach, H. (1993). Covariation bias, classical conditioning, and phobic fear. Integrative Physiological and Behavioral Science, 28, $167-170$.

De Jong, P.J., Merckelbach, H., \& Amtz, A. (1991). Illusory correlation, online covariation estimates, and electrodermal responding in a (quasi) conditioning paradigm. Biological Psychology, 31, 201-212.

De Jong, P.J., Merckelbach, H., \& Amtz, A. (1991). Eyeblink startle responses in spider phobics before and after treatment. Journal of Behavioral Assessment and Psychopathology, 13, 213-223. 
De Jong, P.J., Merckelbach, H., \& Arntz, A. (1992). Eyeblink startle in spider phobics. International Journal of Psychophysiology, 6, 264-265.

De Jong, P.J., Merckelbach, H. \& Amtz, A. (1994). Covariation bias in phobic subjects: The relationship between a priori expectancy, on-line expectancy, autonomic responding, and a posteriori contingency judgment. Jourmal of Abnormal Psychology, in press.

De Jong, P.J., Merckelbach, H., Arntz, A., \& Nijman, H. (1992). Covariation detection in treated and untreated spider phobics. Joumal of Abnormal Psychology, 101, 724-727.

De Jong, P.J., Merckelbach, H., Koertshuis, G., \& Muris, P. (1994). UCSinflation and acquired fear responses in human conditioning. Advances in Behaviour Research and Therapy, in press.

De Jong, P., Merckelbach, H., \& Muris, P. (1989) Conjugate lateral eye movements, cerebral dominance and anxiety. In R.J. Takens (Ed.) European perspectives in psychology; Vol 2, (pp. 38-48) New York: Wiley.

De Jong, P.J., Merckelbach, H., \& Nijman, H. (1994). Hemisphere preference, anxiety, and covariation bias. Manuscript under review.

De Jong, P.J., \& Muris, P. (1994). UCS-inflation, trait anxiety, and monitoring coping style. Manuscript under review.

De Jong, P.J., van Baast, R., \& Arntz, A. (1994). Placebo induced pain reduction: The role of UCS expectancies and UCS experiences. Manuscript under review.

De Jong, P.J., van den Hout, M.A., \& Merckelbach, H, (1994). Covariation bias and the return of fear. Behaviour Research and Therapy, in press.

Merckelbach, H., Amtz, A., de Jong, P., \& Schouten, E. (1993). Effects of endorphin blocking on conditioned SCR in humans. Behaviour Research and Therapy, 31, 775-779.

Merckelbach, H., Arntz, A, \& de Jong, P. (1991). Conditioning experiences in spider phobics. Behaviour Research and Therapy, 29, 333-335.

Merckelbach, H., Arntz, A., Arrindell, W., \& de Jong P. (1992). Pathways to spider phobia. Behaviour Research and Therapy, 30, 543-546.

Merckelbach, H. \& de Jong, P. (1988). Geconditioneerde inhibitie van psychofysiologische reacties op pijnprikkels: feiten en speculaties. Psychologica Belgica, 28, 139-155.

Merckelbach, H. \& de Jong. P.J. (1992). Fobieën, conditionering en UCSinflatie. Gedragstherapie, 25, 93-106.

Merckelbach, H. \& de Jong, P.J. (1994). Waarom Orlemans moet blijven. Directieve Therapie.

Merckelbach, H., de Jong P. \& Amtz, A. (1991). Imagery ability and exposure in vivo in spider phobia. Behaviour Research and Therapy, 29, 203-205.

Merckelbach, H., de Jong, P.J., Arntz, A., \& Schouten, E. (1993). The role of evaluative learning and disgust sensitivity in the etiology and treatment of spider phobia. Advances in Behaviour Research and Therapy, 15, 243255.

Merckelbach, H., de Jong, P.J., \& van den Hout, M.A. (1994). Twee vormen van conditionering, twee vormen van therapie? Gedragstherapie, in druk. 
Merckelbach, H., Dijkstra, A., de Jong, P.J., \& Muris, P. (1994). No effects of verbal verus imaginal cognitive strategies on emotional responses to aversive slides. Behavioural and Cognitive Psychotherapy, in press.

Merckelbach, H., Muris, P. \& de Jong, P. (1990). Hemisphere preference, phobia, and depression. International Journal of Neuroscience, 55, 119123.

Merckelbach, H., Muris, P. \& de Jong, P. (1990). De paradox van verdringing. Psychologie, 7, 23-25.

Murckelbach, H., Muris, P., van den Hout, M.A. \& de Jong, P. (1991). Rebound effects of thought suppression: Instruction-Dependent? Behavioural Psychotherapy, 19, 225-238.

Merckelbach, H., van den Hout, M.A. \& de Jong, P.J. (1989). Psychometric and electrodermal aspects of illusory correlations: Paradoxical results. Psychologica Belgica, 29, 135-148.

Merckelbach, H., van Hout, W., de Jong, P.J. \&, van den Hout, M.A. (1990). Classical conditioning and attentional bias. Journal of Behavior Therapy and Experimental Psychiatry, 21, 185-191.

Mulkens, S., de Jong, P.J., \& Merckelbach, H. (1994). Disgust sensitivity: A vulnerability factor for spider phobia. Manuscript under review.

Muris, P., \& de Jong, P.J. (1993). Monitoring and perception of threat. Personality and Individual Differences, 15, 467-470.

Muris, P., de Jong, P.J., Merckelbach, H. \& van Zuuren, F.J. (1993a). Monitoring coping style and exposure outcome in spider phobics. Behavioural and Cognitive Psychotherapy, 21, 329-333.

Muris, P., de Jong, P.J., Merckelbach, H., \& van Zuuren, F. (1993b). Is exposure therapy outcome affected by a monitoring coping style? Advances in Behaviour Research and Therapy, 15, 291-300.

Muris, P., de Jong, P.J., van Zuuren, F., \& Merckelbach, H. (1994). Effects of imposed monitoring and blunting strategies on emotional reactivity. Anxiety, Stress, and Coping, 7, 53-65.

Muris, P., Merckelbach, H., \& de Jong, P.J. (1993). Verbalization and environmental cuing in thought suppression. Behaviour Research and Therapy, $31,609-612$.

Muris, P., Merckelbach, H., \& de Jong, P.J. (1994). Exposure therapy outcome in spider phobics: Effects of monitoring and blunting coping styles. Manuscript under review.

Muris, P., Merckelbach, H., van den Hout, M.A. \& de Jong, P. (1992). Suppression of emotional and neutral material. Behaviour Research and Therapy, 30, 639-642.

Muris, P., van Zuuren, F., de Jong, P.J., de Beurs, E., \& Hanewald, F. (1994). Monitoring and blunting coping styles: The MBSS and its correlates. and the development of an alternative questionnaire. Personality and Individual Differences, 17, 9-19. 
